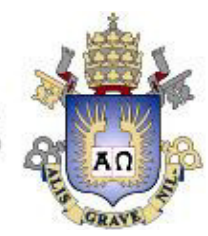

Lucas Lisbôa Vignoli

\title{
Um Estudo do Efeito de Concentração de Tensão em Materiais Anisotrópicos Aplicado à Compósitos Laminados \\ Unidirecionais
}

\section{Dissertação de Mestrado}

Dissertação apresentada como requisito parcial para obtenção do título de Mestre pelo Programa de PósGraduação em Engenharia Mecânica da PUC-Rio.

Orientador: Prof. Jaime Tupiassú Pinho de Castro

Rio de Janeiro

Março de 2016 


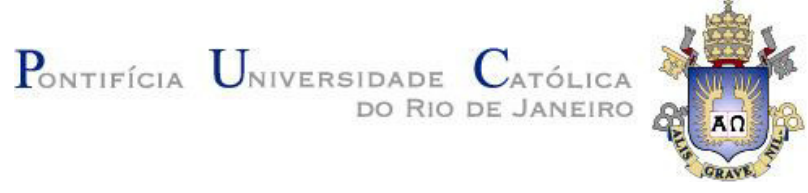

Lucas Lisbôa Vignoli

\section{Um Estudo do Efeito de Concentração de Tensão em Materiais Anisotrópicos Aplicado à Compósitos Laminados \\ Unidirecionais}

Dissertação apresentada como requisito parcial para obtenção do título de Mestre pelo Programa de PósGraduação em Engenharia Mecânica da PUC-Rio. Aprovada pela Comissão Examinadora abaixo assinada.

Prof. Jaime Tupiassú Pinho de Castro Orientador

Departamento de Engenharia Mecânica - PUC-Rio

Prof. Marco Antonio Meggiolaro

Departamento de Engenharia Mecânica - PUC-Rio

Prof. José Roberto Moraes d'Almeida Departamento de Química e de Materiais - PUC-Rio

Prof. Paulo Pedro Kenedi Departamento de Engenharia Mecânica - CEFET/RJ

Prof. Márcio da Silveira Carvalho

Coordenador(a) Setorial do Centro Técnico Científico - PUC-Rio 
Todos os direitos reservados. É proibida a reprodução total ou parcial do trabalho sem autorização da universidade, do autor e do orientador.

\section{Lucas Lisbôa Vignoli}

Graduou-se em Engenharia Mecânica pelo Centro Federal de Educação Tecnológica Celso Suckow da Fonseca (CEFET/RJ) em 2014. Tem atuado majoritariamente na área de Mecânica dos Sólidos com interesse principal em materiais anisotrópicos.

Ficha Catalográfica

Vignoli, Lucas Lisbôa

Um estudo do efeito de concentração de tensão em materiais anisotrópicos aplicado à compósitos laminados unidirecionais / Lucas Lisbôa Vignoli ; orientador: Jaime Tupiassú Pinho de Castro. -2016.

170 f. : il. color. ; $30 \mathrm{~cm}$

Dissertação (mestrado)-Pontifícia Universidade Católica do Rio de Janeiro, Departamento de Engenharia Mecânica, 2016.

Inclui bibliografia

1. Engenharia mecânica - Teses. 2. Concentração de tensão. 3. Laminados unidirecionais. 4. Formalismo de Stroh. 5. Critérios de falha. I. Castro, Jaime Tupiassú Pinho de. II. Pontifícia Universidade Católica do Rio de Janeiro. Departamento de Engenharia Mecânica. III. Título.

CDD: 621 
À minha família e à minha namorada. 


\section{Agradecimentos}

À Igreja, por sempre aliar a razão e a fé na busca da verdade e manter instituições como a PUC-Rio.

À PUC-Rio, em especial ao DEM, e ao CNPq, pelas bolsas de estudo.

Aos meus pais e à minha namorada, pela paciência e incentivo.

Ao Professor Jaime, pela inestimável ajuda durante a orientação e pela confiança depositada.

Ao Professor Marco Antonio, pelas valiosas sugestões. 


\section{Resumo}

Vignoli, Lucas Lisbôa; Castro, Jaime Tupiassú Pinho de. Um Estudo do Efeito de Concentração de Tensão em Materiais Anisotrópicos Aplicado à Compositos Laminados Unidirecionais. Rio de Janeiro, 2016. 170p. Dissertação de Mestrado - Departamento de Engenharia Mecânica, Pontifícia Universidade Católica do Rio de Janeiro.

Entalhes e mudanças bruscas de geometria são indispensáveis na prática, mas geram uma perturbação no campo de tensões e são responsáveis pela falha da maioria dos componentes estruturais. O presente trabalho tem por objetivo estudar o efeito de concentração de tensão em materiais compósitos. O formalismo de Stroh é utilizado para obter a solução analítica da distribuição de tensão na borda de furos elípticos em placas infinitas anisotrópicas sob tensões nominais aplicados genéricas no plano. A teoria clássica dos laminados é aplicada para obter propriedades equivalentes de laminados simétricos de tal forma que o mesmo possa ser considerado uma placa ortotrópica homogênea de rigidez equivalente. Os critérios de Tsai-Wu, Puck e LaRC05 são estudados pelos seus destacados desempenhos no WWFE (World-Wide Failure Exercise) e aplicados a diversas condições de carregamentos para furos circulares e elípticos para diferentes laminados. O estado multiaxial da distribuição de tensões na borda do furo causado pelo efeito da espessura é estudado analiticamente considerando a hipótese limite de deformação plana. A análise de placas finitas é realizada utilizando o software comercial de elementos finitos ANSYS $^{\odot}$ considerando-se tensão plana para comparar soluções aproximadas para as mesmas encontradas na literatura. Por último, um estudo com base na micromecânica utilizando o modelo de Halpin-Tsai para estimar as propriedades de uma lâmina em função da fração volumétrica das fibras é apresentado para avaliar a importância da mesma na concentração de tensão.

\section{Palavras-chave}

concentração de tensão; laminados unidirecionais; formalismo de Stroh; critérios de falha 


\section{Abstract}

Vignoli, Lucas Lisbôa; Castro, Jaime Tupiassú Pinho de (Advisor). A Study of Stress Concentration Effects in Anisotropic Materials Applied to Unidirectional Laminate Composites. Rio de Janeiro, 2016. 170p. MSc Dissertation - Departamento de Engenharia Mecânica, Pontifícia Universidade Católica do Rio de Janeiro.

Notches and abrupt geometry variations are unavoidable in practice, but they result in stress field irregularities and are the reason for failure in majority of structural components. The aim of the present work is to study stress concentration on composite materials. To accomplish, the Stroh formalism is introduced to obtain the analytical solution of the stress distribution around the border an elliptical hole in an infinity plate subjected to general in-plane applied nominal stresses. The classical laminate theory is used to obtain equivalent properties of symmetric laminates since it could be modeled as a homogeneous plate with equivalent stiffness. Tsai-Wu, Puck and LaRC05 criteria are discussed in detail and applied for different load conditions for laminate plates with circular and elliptical holes. The multiaxial stress distribution along the hole border caused by the thickness effect is studied using the plane strain hypothesis. Finite plates are analyzed using the commercial finite element package ANSYS $^{\odot}$ considering plane stress hypothesis to compare the approximation solutions available in literature. At last, a micromechanics based approach using the Halpin-Tsai model to estimate the lamina mechanical properties according to the fiber volumetric fraction is presented to evaluate its influence on stress concentration.

\section{Keywords}

stress concentration; unidirectional laminates; Stroh formalism; failure criteria 


\section{Sumário}

1. Introdução 18

1.1. Revisão Bibliográfica $\quad 19$

1.2. Organização 26

2. Teoria da elasticidade para materiais anisotrópicos 30

2.1. Formalismo de Stroh 30

2.2. Teoria Clássica dos Laminados 45

3. Critérios de falha para laminados unidirecionais 49

3.1. Critério de Tsai-Wu 51

3.1.1. Caso geral 51

3.1.2. Tensão Plana 54

3.2. Critério de Puck 57

3.2.1. Caso geral $\quad 57$

3.2.2. Tensão Plana 61

3.3. Critério LaRC05 67

3.3.1. Caso geral 67

3.3.2. Tensão Plana 73

3.4. Comparação entre os envelopes de falha 78

$\begin{array}{ll}\text { 3.5. } & \text { Estimativa da resistência de laminados }\end{array}$

4. Efeito de furos em placas sob tensão plana 84

4.1. Estimativas de resistências para laminados $[\alpha]_{n}$ com furo circular 89

4.2. Estimativas de resistências para laminados $[ \pm \alpha]_{n s}$ com furo circular 106

4.3. Estimativas de resistências para laminados $[\alpha]_{n}$ com furo elíptico 116

5. Efeito de furos em placas sob deformação plana 132

6. Estudo de outros parâmetros afetam a concentração de tensão

6.1. Estudo de placas finitas 142

6.2. Uma breve abordagem sobre o efeito das fibras na concentração de tensão

$\begin{array}{ll}\text { 7. Conclusões } & 150\end{array}$

8. Referências bibliográficas 153

APÊNDICE A - Concentração de Tensão em uma Placa Anisotrópica com Furo Circular 
APÊNDICE B - Relação Entre Notações 162

ANEXO I - Lista de Publicações $\quad 164$

ANEXO II - Envelopes de Falha do WWFE 165 


\section{Lista de Figuras}

Figura 1 Exemplos de aplicações de materiais compósitos: (a) pás de turbina; (b) reforço estrutural de colunas; (c) turbinas eólicas. (CHAWLA, 2012

Figura 2 Exemplo da propagação do dano paralelamente à direção das fibras (KAMAN, 2011)

Figura 3 Concentração de tensão gerada pelo efeito das fibras na matriz (DANIEL; ISHAI, 1994)

Figura 4 Eixos de coordenadas utilizados para modelar o furo elíptico 36

Figura 5 Principais envelopes de falha pelo modelo de Tsai-Wu 54

Figura 6 Influência do parâmetro $a_{1122}^{*}$ no envelope de falha de Tsai-Wu

Figura 7 Efeito da terceira componente de tensão (para tensão plana) nos envelopes de falha de Tsai-Wu 56

$\begin{array}{lll}\text { Figura } 8 & \text { Plano crítico no modelo de falha da matriz } & 59\end{array}$

$\begin{array}{lll}\text { Figura } 9 & \text { Modos de falha da matriz para tensão plana } & 62\end{array}$

Figura 10 Principais envelopes de falha pelo modelo de Puck 63

Figura 11 Influência do parâmetro $n_{i t}$ no envelope de falha de Puck 64

Figura 12 Influência dos parâmetros $p_{12}^{c}$ e $p_{12}^{t}$ no envelope de falha de Puck 65

Figura 13 Efeito da terceira componente de tensão (para tensão plana) nos envelopes de falha de Puck $\quad 66$

Figura 14 Plano crítico considerando o desalinhamento das fibras 69

$\begin{array}{lll}\text { Figura 15 } & \text { Principais envelopes de falha pelo modelo de LaRC05 } & 74\end{array}$

Figura 16 Influência do parâmetro $b_{l}$ no envelope de falha de LaRC05 75

Figura 17 Círculo de Mohr para compressão uniaxial perpendicular às fibras 76

Figura 18 Efeito da terceira componente de tensão (para tensão plana) no envelope de falha LaRC05

Figura 19 Comparação entre os envelopes de falha 78

Figura 20 Variação das propriedades dos laminados $[\alpha]_{n}$ e $[ \pm \alpha]_{n s} \quad 80$

Figura 21 Comparação da estimativa da resistência à tração e à compressão de $\begin{array}{ll}\text { laminados }[\alpha]_{n} \text { e }[ \pm \alpha]_{n s} & 82\end{array}$

Figura 22 Ilustração do efeito da condição de simetria $\quad 84$

Figura 23 Razão entre a distribuição da tensão tangencial na borda do furo e a tensão nominal $\left(\sigma_{11}^{(l)} / \sigma_{n}\right)$ para uma placa com furo circular e diversos valores de $\alpha$ sob diferentes condições de carregamento $\quad 85$

Figura 24 Diferença entre os carregamentos de cisalhamento puro e tensão/compressão $\quad 88$

Figura 25 Tensões ao longo da borda do furo em coordenadas do material para uma placa grande de laminado unidirecional com furo circular e carregada uniaxialmente

Figura 26 Estimativas de $S_{F P F}^{t} / S_{11}^{t}$ e $S_{F P F}^{c} / S_{11}^{c}$ para placas grandes de laminados unidirecionais com furos circulares

Figura 27 Variação das funções de falha na borda do furo para o carregamento de tração uniaxial com $\sigma_{11}^{(g)}=90 M P a$ e $\alpha=0^{\circ}$ no laminado unidirecional 
Figura 28 Variação das funções de falha na borda do furo para o carregamento de compressão uniaxial com $\sigma_{11}^{(g)}=-70 M P a$ e $\alpha=15^{\circ}$ no laminado unidirecional

Figura 29 Variação das funções de falha na borda do furo para o carregamento de compressão uniaxial com $\sigma_{11}^{(g)}=-60 M P a$ e $\alpha=75^{\circ}$ no laminado unidirecional

Figura 30 Tensões ao longo da borda do furo em coordenadas do material para uma placa grande de laminado unidirecional com furo circular e carregada por cisalhamento puro

Figura 31 Estimativas de $S_{F P F}^{s} / S_{12}$ para placas grandes de laminados unidirecionais com furos circulares

Figura 32 Estimativas de $S_{F P F}^{t} / S_{22}^{t}$ e $S_{F P F}^{c} / S_{22}^{c}$ para placas grandes de laminados unidirecionais com furos circulares

Figura 33 Variação das funções de falha na borda do furo para o carregamento de
cisalhamento puro com $\sigma_{12}^{(g)}=42.5 M P a$ e $\alpha=30^{\circ}$ no laminado unidirecional 100

Figura 34 Variação das funções de falha na borda do furo para o carregamento de cisalhamento puro com $\sigma_{12}^{(g)}=50 M P a$ e $\alpha=45^{\circ}$ no laminado unidirecional 100

Figura 35 Tensões ao longo da borda do furo em coordenadas do material para uma placa grande de laminado unidirecional com furo circular e carregada biaxialmente

Figura 36 Variação das funções de falha na borda do furo para o carregamento de biaxial com $\sigma_{11}^{(g)}=\sigma_{22}^{(g)}=15 M P a$ e $\sigma_{11}^{(g)}=\sigma_{22}^{(g)}=17.5 M P a$ para qualquer valor de $\alpha$ no laminado unidirecional

Figura 37 Variação das funções de falha na borda do furo para o carregamento de biaxial com $\sigma_{11}^{(g)}=\sigma_{22}^{(g)}=-60 M P a$ e $\sigma_{11}^{(g)}=\sigma_{22}^{(g)}=-68 M P a$ para qualquer valor de $\alpha$ no laminado unidirecional

Figura 38 Tensões ao longo da borda do furo em coordenadas do material para uma placa grande de laminado angle-ply com furo circular e carregada uniaxialmente

Figura 39 Estimativas de $S_{F P F}^{t} / S_{11}^{t}$ e $S_{F P F}^{c} / S_{11}^{c}$ para placas grandes de laminados angle-ply com furos circulares

Figura 40 Variação das funções de falha na borda do furo para carregamentos uniaxiais no laminado angle-ply com: $\sigma_{11}^{(g)}=90 \mathrm{MPa}$ e $\alpha=0^{\circ}$; $\sigma_{11}^{(g)}=-70 M P a$ e $\alpha=15^{\circ}$; e $\sigma_{11}^{(g)}=-60 M P a$ e $\alpha=75^{\circ}$

Figura 41 Tensões ao longo da borda do furo em coordenadas do material para uma placa grande de laminado angle-ply com furo circular e carregada com cisalhamento puro

Figura 42 Estimativas de $S_{F P F}^{s} / S_{12}$ para placas grandes de laminados angle-ply com furos circulares

Figura 43 Variação das funções de falha na borda do furo para carregamentos 
cisalhantes de $\sigma_{12}^{(g)}=8.5 \mathrm{MPa}$ no laminado angle-ply para lâminas com $\alpha= \pm 45^{\circ}$

Figura 44 Variação de $\sigma_{11}$ ao longo da borda do furo placa grande de laminados unidirecionais com furo elítico $\left(r_{a} / r_{b}=2\right)$ e carregada uniaxialmente

Figura 45 Variação de $\sigma_{22}$ ao longo da borda do furo placa grande de laminados unidirecionais com furo elítico $\left(r_{a} / r_{b}=2\right)$ e carregada uniaxialmente

Figura 46 Variação de $\sigma_{12}$ ao longo da borda do furo placa grande de laminados unidirecionais com furo elítico $\left(r_{a} / r_{b}=2\right)$ e carregada uniaxialmente

Figura 47 Estimativas de $S_{F P F}^{t} / S_{11}^{t}$ para placas grandes de laminados unidirecionais com furos elípticos

Figura 48 Estimativas de $S_{F P F}^{c} / S_{11}^{c}$ para placas grandes de laminados unidirecionais com furos elípticos

Figura 49 Diferentes tipos de falhas de um laminado: (a) início do dano em regiões diferentes; (b) ruptura frágil; (c) pull-out; (d) delaminação (adaptada de Hallett et al., 2009)

Figura 50 Diferentes modos de progressão da falha em uma lâmina com furo circular de acordo com a direção de laminação: (a) $0^{\circ}$; (b) $45^{\circ}$; (c) $90^{\circ}$; (d) $-45^{\circ}$ (adaptada de Nikishkov et al., 2015)

Figura 51 Variação do fator de restrição transversal na borda do furo para o caso de deformação plana

Figura 52 Tensões ao longo da borda do furo em coordenadas do material para uma placa grande de laminado unidirecional com furo circular e carregada uniaxialmente considerando a hipótese de deformação plana

Figura 53 Comparação entre as estimativas de $S_{F P F}^{t} / S_{11}^{t}$ e $S_{F P F}^{c} / S_{11}^{c}$ para placas grandes de laminados unidirecionais com furos circulares sob estado plano de tensões e estado plano de deformações

Figura 54 Casos selecionados para carregamentos uniaxiais em uma placa grande com furo circular assumindo deformação plana

Figura 55 Razão entre as resistências estimadas considerando deformação plana e $\begin{array}{ll}\text { tensão plana } & 140\end{array}$

Figura 56 Regiões utilizadas para parametrizar a malha 144

Figura 57 Exemplo de malha utilizada para $d / H=0.5$

Figura 58 Comparação entre as estimativas para placas finitas utilizando o modelo proposto por Tan e as soluções utilizando o MEF 145

Figura 59 Variação das propriedades mecânicas da lâmina de acordo com a fração $\begin{array}{ll}\text { volumétrica de fibras } & 148\end{array}$

Figura 60 Influência da fração volumétrica de fibras na concentração de tensão ao $\begin{array}{ll}\text { longo da borda do furo } & 149\end{array}$

Figura 61 Envelopes de falha do WWFE I (SODEN et al., 2004 e HINTON et al., 2004) 165

Figura 62 Envelopes de falha do WWFE II (Kaddour e Hinton, 2013) 167 


\section{Lista de Tabelas}

Tabela 1 Propriedades mecânicas da lâmina com $\mathrm{V}^{(\mathrm{f})}=60 \% \quad 28$

Tabela 2 Propriedades mecânicas da matriz 29

Tabela 3 Propriedades mecânicas das fibras 29

Tabela 4 Parâmetros das malhas convergentes 144 


\title{
Lista de Símbolos
}

\author{
$a_{i j}, a_{i j k l}$ \\ tensores simplificados do modelo de Tsai-Wu \\ A \\ matriz que relaciona as forças e as deformações no plano \\ médio na Teoria Clássica dos Laminados \\ $b_{T}, b_{L}$ \\ parâmetros ajustáveis do modelo LaRC05 \\ B \\ matriz que acopla os efeitos de forças com curvaturas e de \\ momentos com deformações no plano médio na Teoria \\ Clássica dos Laminados \\ tensor de flexibilidade \\ diâmetro do furo \\ D \\ matriz que relaciona os momentos e as curvaturas no plano \\ na Teoria Clássica dos Laminados \\ $\mathbf{e}_{1}^{(l)}, \mathbf{e}_{2}^{(l)}$ \\ vetores unitários$$
E_{1}, E_{2}, E_{3}
$$ \\ módulos de elasticidade (os índices subscritos indicam a \\ direção)
}

$E^{(f)}, E_{1}^{(f)}, E_{2}^{(f)}$

módulos de elasticidade das fibras (os índices subscritos indicam a direção)

$E^{(m)}$

módulos de elasticidade da matriz

$f(z)$

$\mathbf{f}(z)$

função utilizada para descrever a solução geral do problema de elasticidade

matriz das funções que garantem as condições de contorno 


$$
\begin{array}{ll}
f_{L}^{(f, t)}, f_{L}^{(f, c)}, f_{L}^{(m)} & \text { funções de falha de LaRC05 } \\
f_{P}^{(f, t)}, f_{P}^{(f, c)}, f_{P}^{(m, t)}, f_{P}^{(m, c)} & \text { funções de falha de Puck } \\
f_{T W} & \text { função de falha de Tsai-Wu } \\
\boldsymbol{F} & \text { vetor de força por unidade de comprimento } \\
\mathbf{G}_{1}^{(l)}, \mathbf{G}_{3}^{(l)} & \text { matrizes criadas para simplificar a solução } \\
G_{12}, G_{13}, G_{23} & \text { módulos de elasticidade ao cisalhamento } \\
G^{(f)}, G_{12}^{(f)} & \text { módulos de elasticidade ao cisalhamento das }
\end{array}
$$

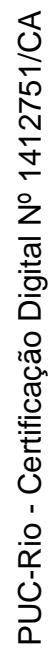$$
G^{(m)}
$$$$
\text { módulos de elasticidade ao cisalhamento da matriz }
$$$$
H
$$

largura da placa finita

tensor de Barnett-Lothe

matriz identidade $3 \times 3$

fator de concentração de tensão

tensor de Barnett-Lothe

parâmetro criado por Puck para estimar o efeito das propriedades das fibras e da matriz

$M$

vetor de momento por unidade de comprimento

$n_{i t}$ expoente que quantifica a interação da tensão normal na direção das fibras na falha da matriz no modelo de Puck $\mathbf{N}, \mathbf{N}^{(l)}$ matriz fundamental da elasticidade em coordenadas do material e local 


$$
\begin{aligned}
& \mathbf{N}_{1}, \mathbf{N}_{2}, \mathbf{N}_{3}, \mathbf{N}_{1}^{(l)}, \mathbf{N}_{2}^{(l)}, \mathbf{N}_{3}^{(l)} \quad \text { componentes da matriz fundamental da elasticidade em } \\
& \text { coordenadas do material e local } \\
& O_{i j}, O_{i j k l}^{\prime} \quad \text { tensores do modelo de Tsai-Wu para tensões } \\
& p \quad \text { autovalor do material } \\
& p_{\Phi}^{t}, p_{\Phi}^{c} \quad \text { parâmetros ajustáveis do modelo de Puck } \\
& \mathbf{Q}, \mathbf{Q}^{(l)} \quad \text { matrizes (em coordenadas do material e local) do formalismo } \\
& \text { de Stroh para obter a equação dos autovalores } \\
& \text { maior semi-eixo da elipse } \\
& \text { maior semi-eixo da elipse } \\
& \mathbf{R}, \mathbf{R}^{(l)} \text { matrizes (em coordenadas do material e local) do formalismo } \\
& \text { de Stroh para obter a equação dos autovalores } \\
& \text { tensor de rigidez } \\
& \text { tensor de Barnett-Lothe } \\
& \text { resistências da lâmina } \\
& \begin{array}{l}
S_{11}^{t}, S_{11}^{c}, S_{22}^{t} \\
S_{12}, S_{13}, S_{23}
\end{array} \\
& S_{F P F}^{t}, S_{F P F}^{c}, S_{F P F}^{s} \quad \text { resistência ao início do dano para tração, compressão e } \\
& \text { cisalhamento } \\
& S_{12}^{\Phi} \quad \text { parâmetro equivalente à resistência no plano dos } \\
& \text { cisalhamentos no plano crítico } \\
& S_{23}^{(23)} \\
& \text { "resistência equivalente" do cisalhamento no plano crítico } \\
& \mathbf{T}, \mathbf{T}^{(l)} \text { matrizes (em coordenadas do material e local) do formalismo } \\
& \text { de Stroh para obter a equação dos autovalores }
\end{aligned}
$$


$T_{c}$

$u_{i}$

$v$

$\boldsymbol{V}$

$V^{(f)}$

$V^{(m)}$

$x_{i}, x_{i}^{(g)}, x_{i}^{(l)}, x_{i}^{(h)}$

$X_{11}$

$w$

$W$

$Y_{i j}, Y_{i j k l}^{\prime}$

$z$

$\alpha$

$\beta$

$\delta_{i j}$ fator de restrição transversal

componentes do vetor de deslocamento

autovetor do material

matriz dos autovetores

fração volumétrica das fibras

fração volumétrica da matriz

componentes das coordenadas cartesianas nos sistemas do material, global, local e do furo

"resistência equivalente" que quantifica a interação da tensão normal na direção das fibras na falha da matriz no modelo de Puck

vetor utilizado para escrever a solução das funções de tensões matriz utilizada para escrever a solução das funções de tensões

tensores do modelo de Tsai-Wu para deformações

variável que relaciona as componentes cartesianas no plano e o autovalor do material

ângulo entre as coordenadas globais e as coordenadas do material

ângulo entre as coordenadas material e as coordenadas do furo

delta de Kronecker 


\begin{tabular}{|c|c|}
\hline$\varepsilon_{i j}, \varepsilon_{i j}^{(g)}, \varepsilon_{i j}^{(l)}$ & $\begin{array}{l}\text { componentes de tensões coordenadas do material, global e } \\
\text { local }\end{array}$ \\
\hline$\varepsilon^{0}$ & vetor de deformação no plano médio da placa \\
\hline$\phi_{i}$ & componentes das funções de tensões \\
\hline$\gamma$ & ângulo de rotação no plano $x_{2}-x_{3}$ \\
\hline$\Gamma$ & região da borda do furo \\
\hline$\eta_{E_{2}}, \eta_{G_{12}}$ & parâmetros do modelo de Halpin-Tsai \\
\hline$\varphi, \varphi_{0}$ & desalinhamento total e desalinhamento inicial das fibras \\
\hline$\kappa$ & vetor de curvatura \\
\hline$\lambda_{i j}$ & operador utilizado para decompor os tensores \\
\hline$\nu_{12}, \nu_{13}, \nu_{23}$ & coeficientes de Poisson \\
\hline$\nu^{(f)}, \nu_{12}^{(f)}$ & coeficientes de Poisson das fibras \\
\hline$\nu^{(m)}$ & coeficientes de Poisson da matriz \\
\hline$\theta$ & ângulo utilizado para mapear a borda do furo no espaço real \\
\hline$\sigma_{i j}, \sigma_{i j}^{(g)}, \sigma_{i j}^{(l)}$ & $\begin{array}{l}\text { componentes de tensões coordenadas do material, global e } \\
\text { local }\end{array}$ \\
\hline$\tau_{1}, \tau_{2}$ & vetores das tensões aplicadas \\
\hline$\xi$ & $\begin{array}{l}\text { vetor criado para obter a equação dos autovalores da forma } \\
\text { tradicional }\end{array}$ \\
\hline$\xi_{E_{2}}, \xi_{G_{12}}$ & parâmetros ajustáveis do modelo de Halpin-Tsai \\
\hline
\end{tabular}


$\psi$

$\Omega$

$\zeta$

$[\alpha]_{n}$

$[ \pm \alpha]_{n s}$ ângulo utilizado para definir a borda do furo no espaço real

matriz de rotação

representação de um círculo de raio unitário no espaço complexo

laminado unidirecional com ' $n$ ' lâminas

laminado angle-ply com '2n' lâminas e simétrico em relação ao plano central 
“... faith does not enter into conflict with science but co-operates with it, offering fundamental criteria to ensure it promotes universal good, and asking only that science desist from those initiatives that, in opposition to God's original plan, may produce effects which turn against man himself. Another reason for which it is rational to believe is this: if science is a valuable ally of faith in our understanding of God's plan for the universe, faith also directs scientific progress towards the good and truth of mankind, remaining faithful to that original plan."

Papa Emérito Bento XVI, "It is rational to believe" 


\section{Introdução}

O presente trabalho tem o objetivo de estudar o efeito de concentração de tensão em materiais compósitos por meios analíticos, considerando estruturas bidimensionais (2D) com as hipóteses de tensão plana e deformação plana para placas infinitas, e pelo método dos elementos finitos (EF), para avaliar efeitos de placas finitas, e aplicar diferentes critérios de falha existentes para comparar a predição dos mesmos.

Materiais compósitos têm sido largamente utilizados em diversas áreas da indústria pela sua característica de baixo peso específico e alta resistência. O presente texto será focado em laminados unidirecionais pela sua particular anisotropia intrínseca.

A Figura 1 mostra algumas aplicações em diferentes indústrias. Na Figura 1.a são mostradas pás de turbinas aeroespaciais, enquanto na Figura 1.b é mostrada a aplicação de reforço estrutural na construção civil e na Figura 1.c um parque eólico offshore. Nas duas primeiras aplicações são utilizadas fibras de carbono e na terceira de fibra de vidro.

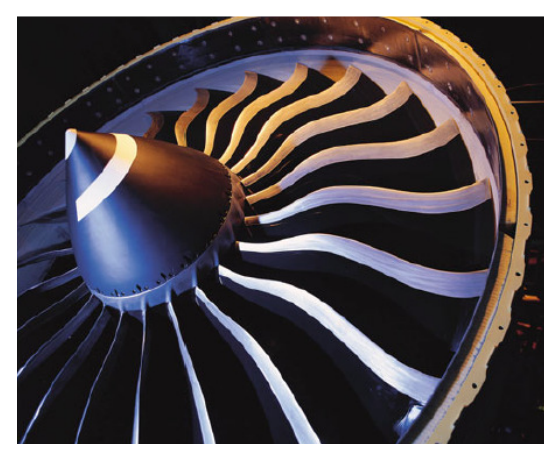

(a)

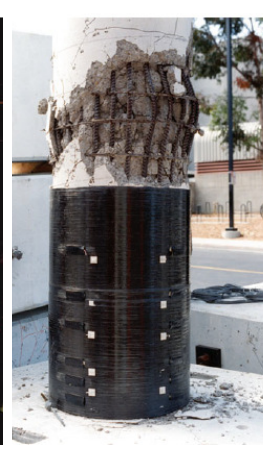

(b)

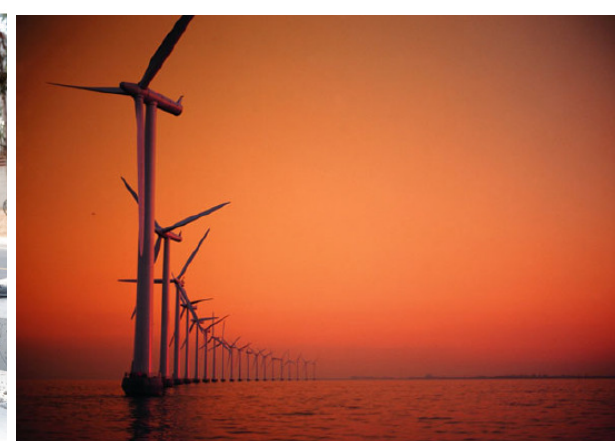

(c)

Figura 1 - Exemplos de aplicações de materiais compósitos: (a) pás de turbina; (b) reforço estrutural de colunas; (c) turbinas eólicas. (CHAWLA, 2012)

Estruturas reais precisam de entalhes, o que gera concentradores de tensões e resultam em pontos críticos dos equipamentos. Focando a análise em matrizes poliméricas, segundo Puck e Schürmann (1998), a falha possui características frágeis, consequentemente não há escoamento aparente e o dano ao redor de entalhes pode gerar a falha repentina. Dessa maneira, torna-se fundamental estimar o dano para cada condição de carregamento.

Todavia, mesmo para carregamentos estáticos, essa não é uma tarefa simples. Na etapa de análise de tensões apenas furos circulares e elípticos em placas infinitas possuem 
soluções exatas, assim como para materiais isotrópicos, mas com o auxilio de métodos numéricos se torna viável obter soluções aproximadas para casos mais complexos. Mesmo com a possível solução dos campos de tensões e deformações, estimar a falha ainda é uma etapa complicada do projeto. Diversos modelos são propostos na literatura, mas apesar de todos os esforços, ainda não há um consenso sobre qual melhor descreve o mecanismo de falha.

\subsection{Revisão Bibliográfica}

Para materiais isotrópicos, a solução clássica da distribuição de tensão em uma placa com furo elíptico foi apresentada por Inglis (1913) e mais tarde Muskhelishvili (1954) apresentou uma solução alternativa com base na técnica de mapeamento conforme (conformal mapping) no espaço complexo.

Para materiais anisotrópicos, pelo menos quatro soluções diferentes da distribuição de tensão em placas grandes com furos são conhecidas: Lekhnitskii (1981), Savin (1970), Green e Zerna (1968) e Hwu e Ting (1989). Nesse ponto, vale ressaltar que as datas citadas não representam as datas de publicação dos artigos originais, sendo então a data cronológica das soluções diferentes, porque alguns dos autores citados (Lekhnitskii e Savin) tiveram suas obras originais publicadas em russo, como citado até por Green e Zerna (1968), dificultando a divulgação da mesma antes da publicação de suas obras em língua inglesa. Apenas as soluções de Lekhnitskii e Hwu e Ting serão discutidas.

Lekhnitskii (1981) propôs uma extensão do trabalho da teoria desenvolvida por Muskhelishvili para aplicação em materiais anisotrópicos, não se limitando apenas a problemas 2D. Segundo o mesmo, a solução geral da distribuição de tensão na borda de um furo elíptico em um material ortotrópico foi obtida em 1961. Lekhnitskii (1987) apresenta uma abordagem similar, mas apenas aplicada a placas. O formalismo de Lekhnitskii é o mais popular no estudo de materiais anisotrópicos, entretanto sua matemática pode se tornar excessivamente exaustiva em alguns problemas, como no caso específico da concentração de tensão.

Hwu e Ting (1989) resolveram o mesmo problema utilizando a abordagem conhecida como o formalismo de Stroh. O formalismo de Stroh tem se tornado mais popular desde a publicação do livro Anisotropic Elasticity: Theory and Applications de 
Ting (1996), que foi o primeiro livro dedicado exclusivamente a discutir o formalismo, juntando então uma abordagem introdutória com uma coleção de problemas já resolvidos utilizando o método. O formalismo de Stroh tem uma base matemática mais elegante e genérica do que o formalismo de Lekhnitskii, podendo ser utilizado para materiais anisotrópicos gerais, não se limitando apenas a materiais ortotrópicos, sem necessitar de grandes alterações. Entretanto, a forma clássica do mesmo é limitado a casos 2D, mas para o estudo de concentração de tensão o torna desvantajoso, visto que a solução 3D não é conhecida. $\mathrm{O}$ formalismo de Stroh será apresentado em detalhe em uma seção específica.

Tan (1987) propôs uma forma de obter fatores de correção para placas ortotrópicas finitas com furo elíptico com maior semi-eixo localizado perpendicular à direção de carregamento da placa e posteriormente (TAN, 1988) a validade da fórmula foi discutida em relação à razão entre os semi-eixos da elipse. Um ponto interessante do estudo é a avaliação do gradiente da tensão na direção perpendicular ao carregamento, partindo da borda do furo. O gradiente de tensões influencia diretamente na propagação de trincas, que normalmente se inicia junto ao entalhe (CASTRO; MEGGIOLARO, 2016a). Todavia, o trabalho também parte do princípio que o ponto de maior concentração de tensão é coincidente ao caso de carregamento similar, mas material isotrópico, o que acaba limitando a abrangência dos resultados.

Weixing e Xinlu (1991) estudaram a concentração de tensão em uma placa retangular com entalhes semi-elípticos laterais de forma numérica pelo método dos $\mathrm{EF}$. Uma limitação conceitual do trabalho é o uso da simetria de um quarto da placa, o que não representa toda a distribuição de tensão para o caso ortotrópico geral em que a direção em que os eixos onde as propriedades mecânicas estão definidas não coincidem com a direção de carregamento. Todavia, mesmo sem ressaltar essa hipótese do modelo, chegou-se a uma equação aproximada para estimar o $\mathrm{K}_{\mathrm{t}}$ em uma placa finita a partir do resultado do formalismo de Lekhnitskii para uma placa infinita.

Toubal et al. (2005) utilizaram um método ótico de medição de campos de deslocamentos para avaliar a concentração de tensão em placas ortotrópicas, o que torna vantajoso em relação ao uso de strain gages que só conseguem dados pontuais. Todavia, de acordo com a comparação entre os resultados numérico, analítico e experimental, o método não foi capaz de medir de forma eficaz o campo de deslocamentos, ou houve alguma não 
linearidade do comportamento do material que não foi levada em conta nos modelos numérico e analítico e não foi explicada.

Soutis e Filiou (1998) estudaram o efeito do carregamento biaxial na distribuição de tensão na borda e na proximidade de um furo circular para laminados. Assim, como grande parte dos modelos presentes na literatura, como os modelos de Whitney e Nuismer (1974), Konish e Whitney (1975), Tan (1987) e Tan (1988), assume-se que o ponto crítico é conhecido e coincide com o tradicional caso isotrópico, e apenas casos em que o ângulo de laminação das camadas é $0^{0}, \pm 45^{\circ}$ e $90^{\circ}$ são avaliados, o que restringe a aplicação do modelo. Berbinau et al. (2001) fizeram uma abordagem similar para estudar furos com preenchimentos, que possui vasta aplicação para reparo de compósitos.

Soriano e Almeida (1999) desenvolveram um estudo experimental do efeito do entalhe na resistência do laminado e compararam os resultados com algumas estimativas tradicionais existentes na literatura: Critério da Tensão Média; Critério da Tensão Pontual; e Critério de Mar-Lin. Segundo os autores, se um laminado possuir entre $40 \%$ e $60 \%$ de suas lâminas orientadas a $0^{0}$, essas lâminas iram comandar o mecanismo de falha e algumas propriedades mecânicas do laminado.

Morais (2000) usou uma modelagem de EF mista, com elementos 3D na área próxima da borda do furo e no resto da placa usou elementos 2D, o que, segundo o mesmo, torna o modelo mais eficiente, e propôs uma abordagem experimental baseada no ajuste de dados experimentais que estima de forma simplificada a resistência à tração de uma placa com furo circular e leva o diâmetro de furo explicitamente em consideração.

Iarve et al. (2005) e Mollenhauer et al. (2006) usaram um modelo de degradação da propriedade mecânica do material aplicado a um programa de EF para estimar o dano causado em placas com furos circulares e avaliar a distribuição das deformações em diferentes níveis de dano. As estimativas foram comparadas com medições experimentais utilizando interferometria de Moiré. A modelagem 3D se mostrou necessária para uma melhor descrição do dano segundo os mesmos.

Green et al. (2007) investigaram a influência do diâmetro do furo na resistência final do laminado de forma experimental e concluíram que a resistência diminui com o aumento do diâmetro do furo. Ensaios não destrutivos foram utilizados para avaliar os 
mecanismos de dano de acordo com o nível de carregamento. Concluiu-se também que os problemas de delaminação são mais representativos para peças mais espessas.

Russo e Zuccarello (2007) propuseram uma abordagem para obter a aproximação da solução pontual na borda de um furo circular de uma placa finita para um laminado, assim como uma aproximação para o gradiente em conjunto com a aplicação do critério de falha proposto por Whitney e Nuismer (1974) que diz que o material irá falhar se a tensão principal máxima a uma distância característica do furo for igual à resistência do material. Como comentado anteriormente, a distribuição de tensões ao longo de toda a borda do furo precisa ser obtida para avaliar o processo de falha, sendo isso uma limitação de grande parte dos trabalhos encontrados na literatura.

Lee e Soutis (2008) fizeram um estudo detalhado sobre efeitos de escala, ou seja, o possível erro gerado por testar CPs padronizados ao invés de estruturas em escalas reais. A discussão, baseada nos resultados experimentais, de cada fator estudado é mostrada de forma detalhada e concluiu-se que a resistência diminui com o aumento do diâmetro do furo e, a princípio, não se altera com o volume e a espessura, mas CPs mais finos podem falhar precocemente por flambagem.

O’Higgins et al. (2008) testaram laminados com fibras de carbono e com fibras de vidro (de alta resistência) sob tração usando corpos de prova (CPs) com e sem furo. Verificou-se que fibras de carbono são mais resistentes e oferecem uma rigidez maior ao laminado, enquanto fibras de vidro conseguem atingir uma maior deformação antes da falha, o que pode ser interessante para aplicações nas quais se deseja absorver energia. Os autores fizeram uma abordagem da sensibilidade ao entalhe de cada laminado (diferentes sequencias de empilhamentos foram analisadas) classificando esse parâmetro como a razão entre a resistência medida no $\mathrm{CP}$ entalhado e a resistência medida no CP sem entalhe. De acordo com os mesmos, os laminados de fibra de carbono são menos sensíveis aos entalhes.

Hallet et al. (2009) deram continuidade ao trabalho de Green et al. (2007) e propuseram uma modelagem de EF usando o LS-Dyna para comparar com as previsões obtidas. O modelo inclui elementos de delaminação entre lâminas e elementos que possibilitam a simulação da separação de dois elementos consecutivos em zonas prédefinidas. 
Shah et al. (2010) mostraram vantagens de simulações numéricas quando comparadas a testes padrões com CPs com entalhes pela diferença de tempo e custo, e principalmente porque, segundo os autores, os ensaios produzem resultados limitados que só são válidos para a condição de carregamento, empilhamento, orientação das lâminas e geometria de entalhe testada, o que aumenta a quantidade de testes necessários; ou seja: para cada alteração de variável é necessário um teste adicional. O critério de Tsai-Wu foi utilizado na modelagem de EF e os envelopes de falha resultantes foram comparados com dados experimentais e com outros critérios de falha mais simples (o uso do critério de Tresca para comparação, especificamente, não foi justificado, visto que o mesmo não é aplicável). O modelo de EF simplesmente desconsidera o elemento que atinge a hipótese de falha e elimina a necessidade de simular a propagação de trincas, o que necessitaria de uma malha mais refinada, iterativa e aumentaria consideravelmente o custo computacional.

Huang et al. (2010) propuseram um novo design para CPs de testes biaxiais que, segundo os autores, resulta em um campo de deformações mais homogêneo do que o CP tradicional, gerando um resultado mais confiável.

Kaman (2011) usou mecânica da fratura para modelar com o ANSYS $^{\odot}$ os resultados obtidos experimentalmente no seu estudo da influência da inclinação da fibra em laminados simétricos (a primeira lâmina foi mantida a $0^{0}$ e o ângulo da segunda foi variado). A Figura 2 mostra a grande tendência da trinca se propagar paralelamente à fibra.

Chen et al. (2013) desenvolveram um modelo de EF para estimar a resistência de laminados com furos e avaliar a influência do tamanho do furo e da espessura (segundo os autores, esse foi o primeiro trabalho a considerar a influência da espessura na tenacidade à fratura translaminar). A importância de usar elementos que consideram uma zona coesiva, dependendo do tipo de fratura, foi demostrada pela comparação do resultado de diferentes elementos. Por último, foi recomendado o uso de diferentes elementos de acordo com o modo de falha esperado.

Kechai et al. (2014) utilizaram elementos baseados na Teoria Clássica dos Laminados (TCL), implementaram uma formulação de EF para avaliar a distribuição de tensão em placas compósitas com furos circulares e aplicaram alguns modelos de falha para estimar o carregamento máximo. Os autores usaram um modelo geométrico de um quarto 
de simetria, o que não representa a realidade para materiais anisotrópicos, como será discutido em detalhe posteriormente.
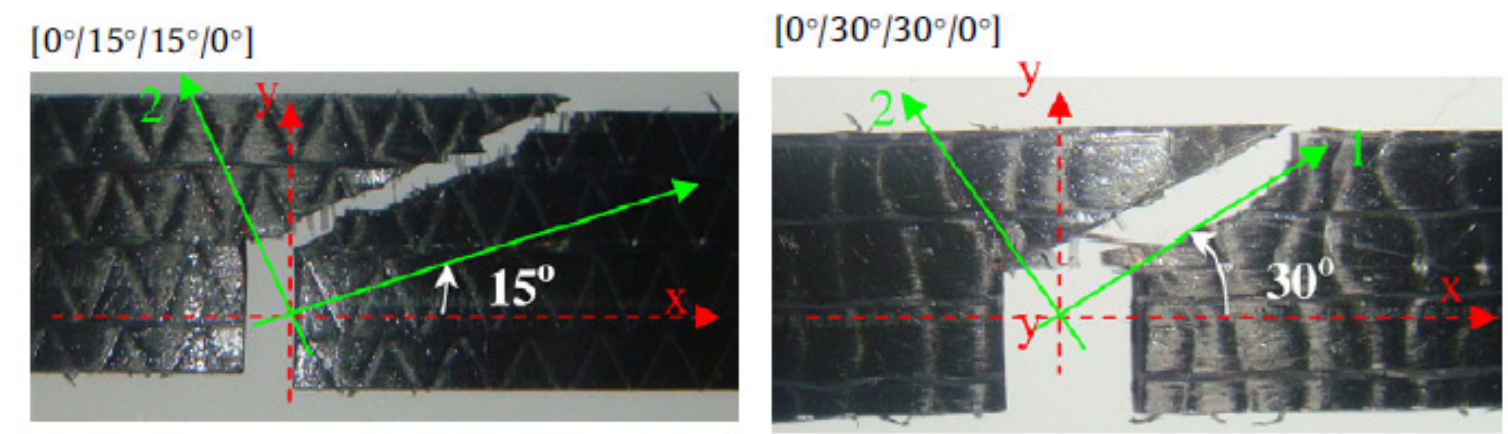

$\left[0^{\circ} / 45^{\circ} / 45^{\circ} / 0^{\circ}\right]$

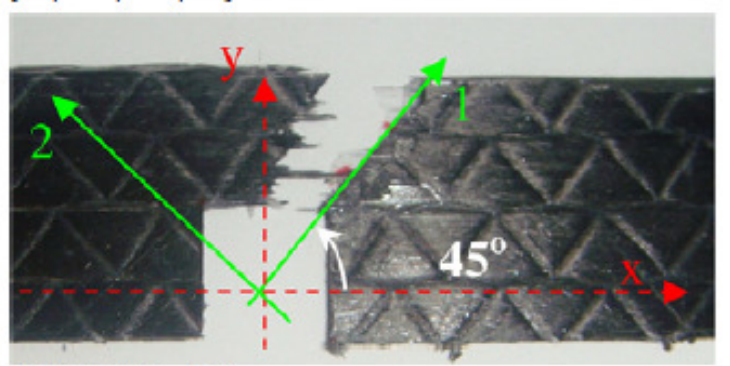

$\left[0^{\circ} / 75^{\circ} / 75^{\circ} / 0^{\circ}\right]$

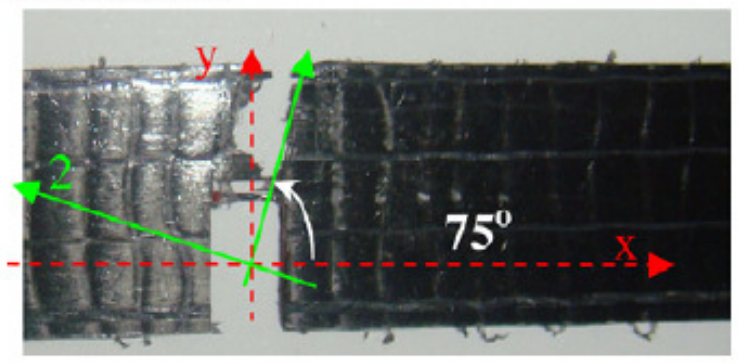

$\left[0^{\circ} / 60^{\circ} / 60^{\circ} / 0^{\circ}\right]$

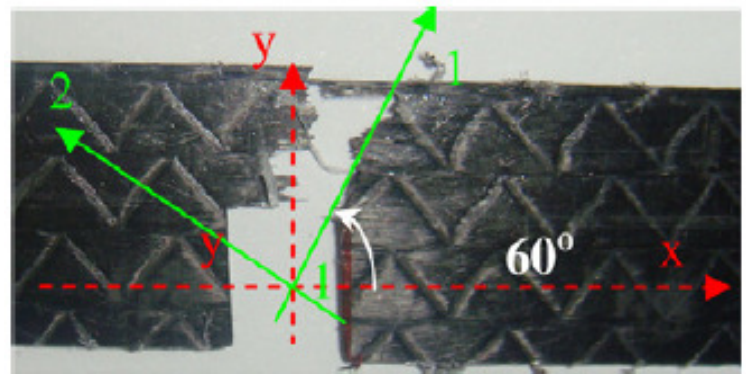

$\left[0^{\circ} / 90^{\circ} / 90^{\circ} / 0^{\circ}\right]$

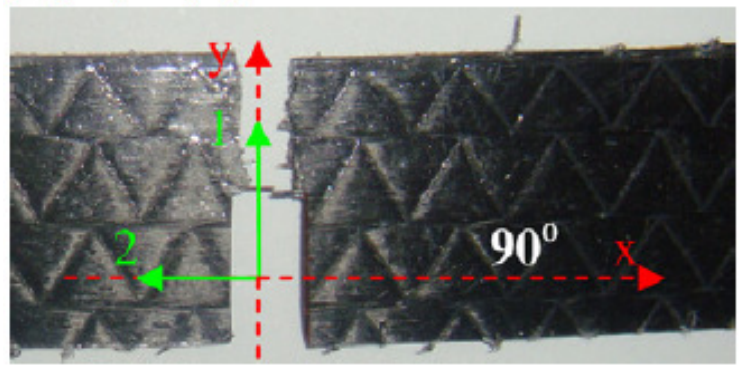

Figura 2 - Exemplo da propagação do dano paralelamente à direção das fibras (KAMAN, 2011).

Sadeghi et al. (2014) propuseram uma modelagem de EF usando sub-rotinas no Abaqus $^{\odot}$ para modelar o dano progressivo na matriz ao redor do entalhe através da lei constitutiva baseado na micromecânica e na energia de deformação.

Sevenois e Koussios (2014) fizeram uma breve revisão sobre alguns métodos analíticos, aplicações de EF e alguns resultados obtidos com abordagens mistas.

$\mathrm{Xu}$ et al. (2014) mostraram dados que indicam mecanismos de dano similares aos observados por Hallet et al. (2009) para outros tipos de entalhes (não circulares).

Kalita et al. (2015) fizeram uma análise de sensibilidade para avaliar a importância das propriedades mecânicas na distribuição de tensões em uma material ortotrópico. 
Segundo os mesmos, os níveis de tensões aumentam quando $E_{2} / E_{1}$ aumentam e $G_{12} / E_{1}$ e $v_{12}$ diminuem.

Kureemun et al. (2015) testaram laminados sob carregamento biaxial de tração/compressão. Os autores reportaram a dificuldade de se testar biaxialmente laminados planos, sem usar CPs tubulares, inclusive por não haver norma existente. Dessa forma, os dados experimentais obtidos se tornam de grande valia e mostram boa compatibilidade com as estimativas numéricas. Uma importante característica do modelo de EF utilizado é o alinhamento da malha com as fibras, o que permite um refinamento onde o dano seja esperado, poupando esforços computacionais, e também uma melhor caracterização dos modelos de falha, visto que os mesmos são separados entre falhas das fibras e da matriz. O efeito da orientação das fibras em relação ao carregamento também foi estudado e mostrouse que a resistência aumenta conforme as fibras e os carregamentos tendem a ficar paralelos.

Moure et al. (2015) desenvolveram uma subrotina de EF usando o Abaqus ${ }^{\circledR}$ para simular a propagação da falha na matriz e falha das fibras em laminados com e sem furos e compararam suas estimativas com diversos valores obtidos na literatura. Três diferentes critérios foram avaliados: de projeto, considera a falha no ponto de tensão máxima da curva tensão/deformação; local, considera a falha no ponto em que a curva tensão deformação para de decair; e macro, considera a falha na deformação máxima antes da ruptura (todos esses pontos são definidos na curva tensão/deformação para uma lâmina a $0^{0}$ ). $\mathrm{O}$ critério local mostrou ser o mais eficaz. Para maior detalhe sobre esses critérios ver a referência original.

Su et al. (2015) estudaram a falha de laminados com furos sob compressão de forma numérica (EF) usando elementos de casca e elementos coesivos para modelar a delaminação. A abordagem foi similar a de Chen et al. (2013) (comentada anteriormente), mas para compressão utilizando o critério de falha de Hashin.

Zappalorto e Carraro (2015) propuseram uma fórmula, baseada em dados experimentais e numéricos, para estimar a concentração de tensão em placas ortotrópicas com diferentes entalhes sob tração. $\mathrm{O}$ valor do $\mathrm{K}_{\mathrm{t}}$ para o mesmo entalhe em um material isotrópico foi tomado como ponto de partida. Apesar das estimativas analíticas serem 
compatíveis com os resultados numéricos e analíticos apresentados, todos os resultados são pontuais e nenhuma distribuição de tensão em toda a borda do furo/entalhe é apresentada.

Uma abordagem micromecânica do efeito de concentração de tensão pode ser também aplicável aos compósitos, e.g., Sabuncuoglu et al. (2014) e Romanov et al. (2015), considerando-os não homogêneos, como mostrado na Figura 3, mas não será parte do presente estudo. Será tomado como base, tanto para análise de tensões quanto para os critérios de falha que serão discutidos mais adiante, que apenas fenômenos em macroescala serão modelados.

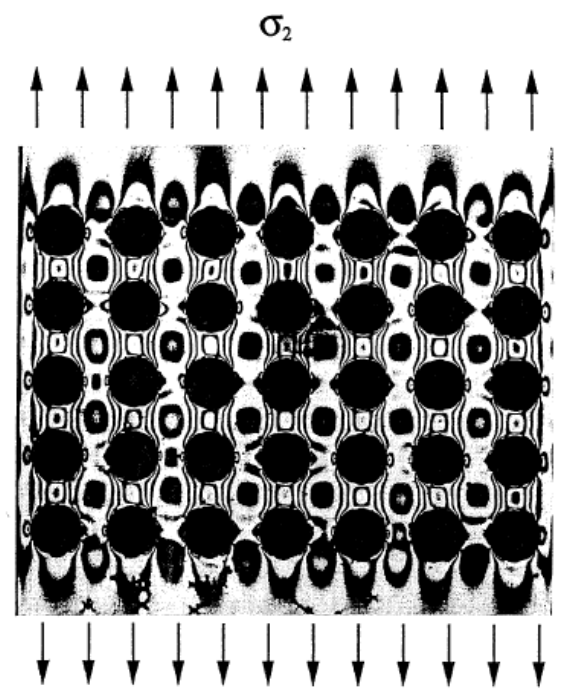

$\sigma_{2}$

Figura 3 - Concentração de tensão gerada pelo efeito das fibras na matriz (DANIEL; ISHAI, 1994)

\subsection{Organização}

Inicialmente será mostrada uma abordagem da teoria da elasticidade para materiais anisotrópicos, mais especificamente utilizando o formalismo de Stroh. Apesar de considerar uma classe mais geral dos materiais do que usualmente abordado em livros clássicos de Teoria da Elasticidade, e.g. Timoshenko e Goodier (1951), segundo Ting (1996), as ferramentas matemáticas utilizadas no desenvolvimento do formalismo de Stroh são tão elegantes e simples que o tornam mais simples do que a clássica abordagem para materiais isotrópicos. Vale ressaltar que o mesmo é valido para qualquer tipo de material com qualquer anisotropia, não sendo restrito às particularidades dos materiais compósitos. 
Posteriormente será introduzida a TCL, um método tradicional e eficiente, baseado na Teoria de Placas, para dividir a parcela de carga entre as lâminas de acordo com o carregamento.

Uma vez que a base analítica necessária para estudar a distribuição de tensões e deformações está posta, serão então introduzidos os conceitos dos critérios de falha. Apenas falhas estáticas serão estudadas. De acordo com Christensen (2005), estimar a falha é um ponto mais árduo durante o projeto de materiais compósitos, sendo essa etapa mais difícil do que obter a distribuição de tensões e deformações, principalmente por causa dos avanços dos métodos numéricos.

Por causa disso, na década de 1990 teve início o World-Wide Failure Exercise (WWFE) com o objetivo de avaliar os diversos modelos propostos para prever a falha de forma imparcial. Basicamente, são selecionados diferentes tipos de laminados (todos unidirecionais com matriz polimérica) e carregamentos e, após testes inicias para medir as propriedades dos materiais, os mesmo dados são fornecidos a todos os grupos participantes. Com esse conjunto de informações (propriedades + carregamentos), cada grupo deve aplicar o seu método/modelo para avaliar o efeito dos carregamentos e estimar algumas respostas do material, como envelopes de falha e deformação, considerando o início e a progressão do dano.

O WWFE-1 teve a intenção de avaliar os principais materiais e carregamentos (HINTON et al., 2004), todavia, devido à grande aplicação dos materiais compósitos, algumas lacunas importantes foram identificadas e concluiu-se a necessidade de haver o WWFE-2, que teve foco no efeito de estados tridimensionais (3D) de tensões (KADDOUR; HINTON, 2012), e o WWFE-3, que ainda não está concluído até o presente momento e visa avaliar o efeito de não-linearidades, como por exemplo concentradores de tensão, carregamentos cíclicos e expansão térmica (KADDOUR et al., 2013). Os critérios de TsaiWu, Puck e LaRC05 foram selecionados para serem estudados em detalhe pelas suas destacadas capacidades de estimar os danos.

Logo em seguida, serão aplicados os três modelos de falha selecionados para casos de concentração de tensão em placas com furos circulares e elípticos para comparar a estimativa de cada critério, juntamente com uma análise de sensibilidade do efeito da 
orientação das fibras em cada lâmina. O estudo de sensibilidade será preferido, ao invés de aplicações de algoritmos de otimização para placas com furos.

Como a abordagem analítica considera que as dimensões da placa sejam consideravelmente maiores do que as do furo, de tal modo que a placa possa ser considerada infinita, o software comercial de Elementos Finitos (EF) ANSYS ${ }^{\circledR}$ será utilizado para avaliar o efeito de placas finitas, considerando ainda o estado plano de tensões e depois o efeito da espessura. O principal objetivo dessa etapa será avaliar modelos aproximados para placas finitas da literatura.

\begin{tabular}{c|c|c}
\multicolumn{3}{c}{ Tabela 1 - Propriedades mecânicas da lâmina com $\mathrm{V}^{(\mathrm{f})}=60 \%$} \\
\hline Propriedades & Unidade & AS carbono/epóxi \\
\hline$E_{1}$ & {$[\mathrm{GPa}]$} & 140 \\
\hline$E_{2}=E_{3}$ & {$[\mathrm{GPa}]$} & 10 \\
\hline$G_{12}=G_{13}$ & {$[\mathrm{GPa}]$} & 6 \\
\hline$G_{23}$ & {$[\mathrm{GPa}]$} & 3.35 \\
\hline$\nu_{12}=\nu_{13}$ & - & 0.3 \\
\hline$\nu_{23}$ & - & 0.49 \\
\hline$E_{1}^{(f)}$ & {$[\mathrm{GPa}]$} & 231 \\
\hline$\nu_{12}^{(f)}$ & - & 0.2 \\
\hline$S_{11}^{t}$ & {$[\mathrm{MPa}]$} & 1990 \\
\hline$S_{11}^{c}$ & {$[\mathrm{MPa}]$} & 1500 \\
\hline$S_{22}^{t}=S_{33}^{t}$ & {$[\mathrm{MPa}]$} & 38 \\
\hline$S_{22}^{c}=S_{33}^{c}$ & {$[\mathrm{MPa}]$} & 150 \\
\hline$S_{12}=S_{13}$ & {$[\mathrm{MPa}]$} & \\
\hline$S_{23}$ & {$[\mathrm{MPa}]$} & \\
\hline
\end{tabular}


Uma breve introdução à micromecânica será apresentada para estudar o efeito da fração volumétrica das fibras na concentração de tensão. Para tal, será utilizado o modelo de Halpin-Tsai para estimar as propriedades mecânicas da lâmina.

Apesar de a metodologia desenvolvida ser genérica, o material estudado em detalhe será o laminado constituído por lâminas de fibras de carbono e matriz de epóxi. As propriedades mecânicas estão mostradas nas Tabelas 1-3.

Tabela 2 - Propriedades mecânicas da matriz

\begin{tabular}{c|c|c}
\hline Propriedades & Unidade & epóxi \\
\hline$E^{(m)}$ & {$[\mathrm{GPa}]$} & 3.2 \\
\hline$G^{(m)}$ & {$[\mathrm{GPa}]$} & 1.2 \\
\hline$\nu^{(m)}$ & - & 0.35 \\
\hline
\end{tabular}

Fonte: Kaddour; Hinton (2012)

Tabela 3 - Propriedades mecânicas das fibras

\begin{tabular}{c|c|c}
\hline Propriedades & Unidade & fibra de carbono \\
\hline$E_{1}^{(f)}$ & {$[\mathrm{GPa}]$} & 231 \\
\hline$E_{2}^{(f)}$ & {$[\mathrm{GPa}]$} & 15 \\
\hline$G_{12}^{(f)}$ & {$[\mathrm{GPa}]$} & 15 \\
\hline$\nu_{12}^{(f)}$ & - & 0.2 \\
\hline
\end{tabular}

Fonte: Kaddour; Hinton (2012) 


\section{Teoria da elasticidade para materiais anisotrópicos}

Esta seção tem o objetivo de fornecer embasamento teórico suficiente para avaliar o efeito de entalhes em laminados unidirecionais. Inicialmente é mostrado o Formalismo de Stroh, que é usado para obter analiticamente o efeito da distribuição de tensões em placas anisotrópicas infinitas com furos elípticos, e em seguida, com a introdução da TCL, sua aplicação em placas compósitas será estudada.

\subsection{Formalismo de Stroh}

O formalismo de Stroh será mostrado a seguir para deduzir a tensão na borda de um furo elíptico de uma placa infinita ortotrópica. Apesar de receber o nome de formalismo de Stroh, o mesmo tem base em três trabalhos das décadas de 1950 e 1960, sendo o primeiro desenvolvido por Eshelby, Read e Shockley e os outros dois por Stroh.

Esses artigos serviram como base para técnicas matemáticas que foram sendo desenvolvidas durante décadas e foram organizadas e publicadas em Ting (1996). Com a publicação do livro, as ideias ficaram expostas de formas organizadas, o que o tornou, então, a fonte bibliográfica única com o Estado da Arte das aplicações do formalismo de Stroh. Posteriormente, Hwu (2009) também publicou um livro com os avanços teóricos mais atuais e algumas aplicações computacionais. Para evitar um número excessivo de citações, todo o desenvolvimento mostrado nessa etapa usará como base Ting (1996) e Hwu (2009) a menos que seja dito o contrário.

Por simplicidade, será assumido o caso bidimensional de tensão plana. Essa hipótese se justifica pelo fato de que o principal objetivo do presente trabalho é estudar o fator de concentração de tensão em placas, todavia posteriormente será avaliado o caso de deformação plana, mas o desenvolvimento matemático é semelhante e devido à praticidade do formalismo, apenas os elementos de algumas matrizes precisam ser alterados.

Inicialmente, serão introduzidos conceitos básicos do formalismo de Stroh considerando como base o sistema de coordenadas do material, que será discutido posteriormente, e nenhum índice será utilizado. A princípio, o material será considerado tendo uma anisotropia genérica, sem nenhuma restrição específica, a menos que seja explicitado o contrário. Como em qualquer problema de elasticidade, as equações de 
equilíbrio (forças de corpo não serão consideradas), a compatibilidade geométrica (considerando a hipótese de pequenos deslocamentos) e a relação constitutiva precisam ser respeitadas e são mostradas nas equações (1.a-c)

$$
\begin{aligned}
& \sigma_{i j, j}=0 \\
& \varepsilon_{i j}=\frac{1}{2}\left(u_{i, j}+u_{j, i}\right) \\
& \sigma_{i j}=s_{i j k l} \varepsilon_{k l}
\end{aligned}
$$

Onde $\sigma_{i j, j}$ é a derivada do tensor da tensão $\sigma_{i j}$ em relação à coordenada $x_{j}, \varepsilon_{i j}$ é o tensor da deformação, $u_{i, j}$ é a taxa de variação do deslocamento $u_{i}$ em relação à coordenada $x_{j}$ e $s_{i j k l}$ é o tensor de rigidez.

Como a base da solução dos problemas utilizando o formalismo de Stroh é descobrir os deslocamentos para cada condição de contorno, vale a pena reescrever a lei constitutiva como função dos deslocamentos através do uso da compatibilidade geométrica, ou seja,

$$
\sigma_{i j}=s_{i j k l}\left[\frac{1}{2}\left(u_{i, j}+u_{j, i}\right)\right]
$$

Expandida a notação indicial e considerando a simetria do tensor, chega-se ao seguinte resultado (lembrando que, como se trata de um problema plano, apenas os deslocamentos no plano serão avaliados, o que significa dizer que $i, j=1,2$ )

$$
s_{i j k l}\left[\frac{1}{2}\left(u_{i, j}+u_{j, i}\right)\right]=s_{i j k l} u_{i, j}
$$

Pode-se, então, reescrever a relação constitutiva como

$$
\sigma_{i j}=s_{i j k l} u_{k, l}
$$

Seguindo o mesmo raciocínio, a equação de equilíbrio pode ser reescrita da seguinte forma

$$
s_{i j k l} u_{k, l j}=0
$$

A solução geral do campo de deslocamento pode então ser expressa como

$$
u_{k}=v_{k} f(z)
$$


com $k=1,2,3$, onde $v_{k}$ são constantes que dependem das propriedades do material e $f(z)$ é uma função genérica que dependerá do material e das condições de contorno, sendo ambas quantidades genéricas que precisam ser descobertas. Para o estudo de problemas bidimensionais, espera-se que a solução dependa apenas das coordenadas $x_{1} \mathrm{e}$ $x_{2}$, de tal forma que pode-se escrever, sem perda de generalidade

$$
z=x_{1}+p x_{2}
$$

Para conseguir a solução de um dado problema, as constantes $v_{k}$ e $p$ precisam ser obtidas e a função $f(z)$ deve ser determinada. Para isso, iremos utilizar as equações de equilíbrio, compatibilidade geométrica e a relação constitutiva obtidas como função do deslocamento. Usando a regra da cadeia para derivar o deslocamento, tem-se

$$
u_{k, l}=v_{k} \frac{d f}{d z} \frac{d z}{x_{l}}=v_{k}\left(\delta_{l 1}+p \delta_{l 2}\right) f^{\prime}(z)
$$

onde $\delta_{i j}=1$ se $i=j$ e $\delta_{i j}=0$ se $i \neq j$ é o delta de Kronecker. Derivando novamente o deslocamento

$$
u_{k, l j}=v_{k}\left(\delta_{l 1}+p \delta_{l 2}\right)\left(\delta_{j 1}+p \delta_{j 2}\right) f^{\prime \prime}(z)
$$

Usando essa relação, a equação de equilíbrio pode ser reescrita como

$$
s_{i j k l} v_{k}\left(\delta_{l 1}+p \delta_{l 2}\right)\left(\delta_{j 1}+p \delta_{j 2}\right) f^{\prime \prime}(z)=0
$$

Expandindo os índices e supondo $f^{\prime \prime}(z) \neq 0$, pode-se chegar à seguinte equação

$$
\left[s_{i 1 k 1}+\left(s_{i 1 k 2}+s_{i 2 k 1}\right) p+s_{i 2 k 2} p^{2}\right] v_{k}=0
$$

Torna-se útil nessa etapa definir as seguintes matrizes

$$
\begin{aligned}
& Q_{i k}=s_{i 1 k 1} \\
& R_{i k}=s_{i 1 k 2} \\
& T_{i k}=s_{i 2 k 2}
\end{aligned}
$$

Logo, a equação (11) pode ser escrita como

$$
\left[Q_{i k}+\left(R_{i k}+R_{k i}\right) p+T_{i k} p^{2}\right] v_{k}=0
$$


Ou de forma matricial

$$
\left[\mathbf{Q}+\left(\mathbf{R}+\mathbf{R}^{T}\right) p+\mathbf{T} p^{2}\right] \boldsymbol{v}=\mathbf{0}
$$

$\mathbf{Q}$ e $\mathbf{T}$ são matrizes $3 \times 3$ positivas definidas pelo fato de que fisicamente a energia de deformação precisa ser positiva. Uma matriz é positiva se todos os seus $n$ possíveis determinantes forem positivos (VAZ et al., 2012). Para que a equação (14) tenha solução não-trivial

$$
\left\|\mathbf{Q}+\left(\mathbf{R}+\mathbf{R}^{T}\right) p+\mathbf{T} p^{2}\right\|=0
$$

Com as equações $(14,15)$ é então possível descobrir os valores de $p$ e $v$ que são denominados como os autovalores e os autovetores do material, respectivamente. Os autovalores do material são sempre números complexos ou imaginários puros; a demonstração dessa afirmação não será feita nesse trabalho, mas pode ser justificada pelo fato de que a energia de deformação é necessariamente positiva.

Conhecendo-se $p$ e $v$, as tensões podem ser obtidas substituindo (8) em (4)

$\sigma_{i j}=\left(s_{i j k 1}+p s_{i j k 2}\right) v_{k} f^{\prime}(z)$

ou

$$
\begin{aligned}
& \sigma_{i 1}=\left(s_{i 1 k 1}+p s_{i 1 k 2}\right) v_{k} f^{\prime}(z)=\left(Q_{i k}+p R_{i k}\right) v_{k} f^{\prime}(z) \\
& \sigma_{i 2}=\left(s_{i 2 k 1}+p s_{i 2 k 2}\right) v_{k} f^{\prime}(z)=\left(R_{k i}+p T_{i k}\right) v_{k} f^{\prime}(z)
\end{aligned}
$$

Avaliando as equações (17.a,b), pode-se definir um novo vetor $w$, tal que

$$
\boldsymbol{w}=\left[\mathbf{R}^{T}+p \mathbf{T}\right] \boldsymbol{v}=-\frac{1}{p}[\mathbf{Q}+p \mathbf{R}] \boldsymbol{v}
$$

Essa igualdade pode ser obtida pela equação dos autovalores e autovetores do material. As tensões podem ser então expressadas, como o uso do novo vetor, como

$$
\begin{aligned}
& \sigma_{i 1}=-p w_{i} f^{\prime}(z) \\
& \sigma_{i 2}=w_{i} f^{\prime}(z)
\end{aligned}
$$

Utilizando as funções de tensão de Airy, as tensões podem ser escritas como

$$
\begin{gathered}
\sigma_{i 1}=-\phi_{i, 2} \\
\sigma_{i 2}=\phi_{i, 1}
\end{gathered}
$$

Pela condição de equilíbrio $\left(\sigma_{12}=\sigma_{21}\right)$ 


$$
\phi_{1,1}+\phi_{2,2}=0
$$

Comparando as equações (20) e (21), pode-se concluir que

$$
\phi_{i}=w_{i} f(z)
$$

Assumindo que os 6 autovalores do material são 3 números complexos distintos e seus conjugados, as soluções gerais do deslocamento e da função de tensão podem ser escritas (lembrando que a soma de um número complexo com seu conjugado é igual a duas vezes a parte real) como

$$
\begin{aligned}
& \mathbf{u}=2 \operatorname{Re}[V \mathbf{f}(z)] \\
& \phi=2 \operatorname{Re}[W \mathbf{f}(z)]
\end{aligned}
$$

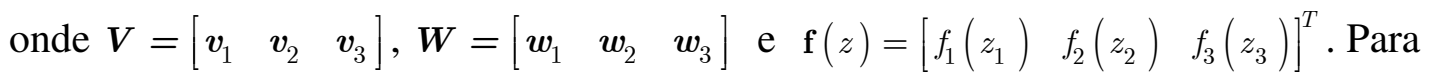
obter a solução completa dos campos de deslocamentos e das funções de tensões, e consequentemente das tensões e deformações, é preciso avaliar as condições de contorno.

$\mathrm{Na}$ etapa anterior foi suposto que o material possuía todos os autovetores diferentes, no entanto, nem todos os materiais obedecem a essa regra. Um exemplo de material que tem todos os autovalores iguais é um material isotrópico. Os materiais que não obedecem a essa regra são chamados de degenerados (degenerate materials).

Os autovalores e autovetores do material podem ser obtidos como mostrado anteriormente, entretanto a forma em que são obtidos não é similar ao problema clássico de autovalores de álgebra linear. Transformar o problema de autovalores para a forma clássica e utilizar os autovetores normalizados possibilitam a utilização de algumas identidades algébricas que tornam o formalismo de Stroh mais prático.

Para transformar a forma do problema, primeiramente vamos escrever as matrizes $\mathbf{Q}, \mathbf{R}$ e $\mathbf{T}$ de forma expandida

$$
\begin{aligned}
\mathbf{Q} & =\left[\begin{array}{lll}
s_{1111} & s_{1121} & s_{1131} \\
s_{2111} & s_{2121} & s_{2131} \\
s_{3111} & s_{3121} & s_{3131}
\end{array}\right] \\
\mathbf{R} & =\left[\begin{array}{lll}
s_{1112} & s_{1122} & s_{1132} \\
s_{2112} & s_{2122} & s_{2132} \\
s_{3112} & s_{3122} & s_{3132}
\end{array}\right]
\end{aligned}
$$




$$
\mathbf{T}=\left[\begin{array}{lll}
s_{1212} & s_{1222} & s_{1232} \\
s_{2212} & s_{2222} & s_{2232} \\
s_{3212} & s_{3222} & s_{3232}
\end{array}\right]
$$

A equação dos autovalores (14) pode ser reescrita das seguintes formas após simples manipulações algébricas

$$
\begin{aligned}
& -\mathbf{Q} \boldsymbol{v}=p\left[\mathbf{R} \boldsymbol{v}+\left(\mathbf{R}^{T}+p \mathbf{T}\right) \boldsymbol{v}\right] \\
& -\mathbf{R}^{T} \boldsymbol{v}-\frac{1}{p}(\mathbf{Q}+p \mathbf{R}) \boldsymbol{v}=p \mathbf{T} \boldsymbol{v}
\end{aligned}
$$

Acrescentando $0 w$ do lado direito da primeira e do lado esquerda da segunda e utilizando a definição do vetor $w$ - equação (18) -, tem-se

$$
\begin{aligned}
& -\mathbf{Q} \boldsymbol{v}+\mathbf{0} \boldsymbol{w}=p[\mathbf{R} \boldsymbol{v}+\mathbf{I} \boldsymbol{w}] \\
& -(\mathbf{R})^{T} \boldsymbol{v}+\mathbf{I} \boldsymbol{w}=p(\mathbf{T} \boldsymbol{v}+\mathbf{0} \boldsymbol{w})
\end{aligned}
$$

onde I é a matriz identidade 3x3. Para transformar as duas equações anteriores em apenas uma, pode-se expressar como a seguinte relação matricial

$$
\left[\begin{array}{cc}
-\mathbf{Q} & \mathbf{0} \\
-\mathbf{R}^{T} & \mathbf{I}
\end{array}\right]\left[\begin{array}{c}
\boldsymbol{v} \\
\boldsymbol{w}
\end{array}\right]=p\left[\begin{array}{cc}
\mathbf{R} & \mathbf{I} \\
\mathbf{T} & \mathbf{0}
\end{array}\right]\left[\begin{array}{c}
\boldsymbol{v} \\
\boldsymbol{w}
\end{array}\right]
$$

Uma identidade útil que pode ser facilmente verificada é

$$
\left[\begin{array}{cc}
\mathbf{0} & \mathbf{T}^{-1} \\
\mathbf{I} & \mathbf{R T}^{-1}
\end{array}\right]\left[\begin{array}{ll}
\mathbf{R} & \mathbf{I} \\
\mathbf{T} & \mathbf{0}
\end{array}\right]=\left[\begin{array}{ll}
\mathbf{I} & \mathbf{0} \\
\mathbf{0} & \mathbf{I}
\end{array}\right]
$$

Vale lembrar que $\mathbf{T}^{-1}$ sempre existe porque $\mathbf{T}$ é positiva definida. Com isso, multiplicando ambos os lados da equação (27) pela matriz mostrada em (28), pode-se escrever

$$
\left[\begin{array}{cc}
-\mathbf{T}^{-1} \mathbf{R}^{T} & \mathbf{T}^{-1} \\
-\mathbf{R} \mathbf{T}^{-1} \mathbf{R}^{T}-\mathbf{Q} & -\mathbf{R T}^{-1}
\end{array}\right]\left[\begin{array}{c}
\boldsymbol{v} \\
\boldsymbol{w}
\end{array}\right]=p\left[\begin{array}{cc}
\mathbf{I} & \mathbf{0} \\
\mathbf{0} & \mathbf{I}
\end{array}\right]\left[\begin{array}{c}
\boldsymbol{v} \\
\boldsymbol{w}
\end{array}\right]
$$

Nesta etapa é útil definir as seguintes matrizes

$$
\begin{aligned}
& \mathbf{N}_{1}=-\mathbf{T}^{-1} \mathbf{R}^{T} \\
& \mathbf{N}_{2}=\mathbf{T}^{-1} \\
& \mathbf{N}_{3}=-\mathbf{R T}^{-1} \mathbf{R}^{T}-\mathbf{Q}
\end{aligned}
$$

Agora é, então, possível rescrever a equação (29) de forma mais compacta como 
$\left[\begin{array}{cc}\mathbf{N}_{1} & \mathbf{N}_{2} \\ \mathbf{N}_{3} & \left(\mathbf{N}_{1}\right)^{T}\end{array}\right]\left[\begin{array}{c}\boldsymbol{v} \\ \boldsymbol{w}\end{array}\right]=p\left[\begin{array}{c}\boldsymbol{v} \\ \boldsymbol{w}\end{array}\right]$

ou

$\mathbf{N} \xi=p \xi$

onde $\xi=\left[\begin{array}{ll}\boldsymbol{v} & \boldsymbol{w}\end{array}\right]^{T}$, o que torna então o problema da forma clássica de autovalor. Pelas definições anteriores, as seguintes propriedades podem ser citadas: $\mathbf{N}_{1}$ e $\mathbf{N}_{3}$ são simétricas; $\mathbf{N}_{2}$ é positiva definida; $-\mathbf{N}_{3}$ é positiva semi-definida; $\mathbf{N}_{1}$ é adimensional; $\mathbf{N}_{2}$ tem unidade $\left[m^{2} / N\right] ; \mathbf{N}_{3}$ tem unidade $\left[N / m^{2}\right]$. Por sua importância no formalismo de Stroh, a matriz $[\mathbf{N}]_{6 \times 6}$ é denominada como matriz fundamental da elasticidade.

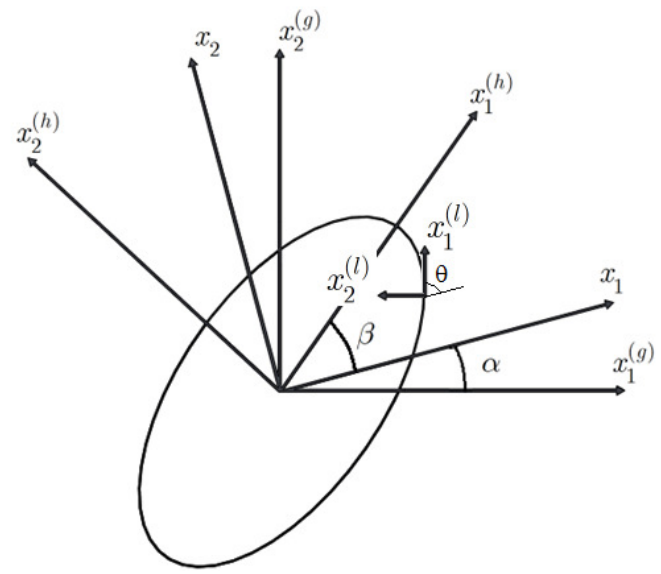

Figura 4 - Eixos de coordenadas utilizados para modelar o furo elíptico

A partir desse ponto, torna-se útil o uso de diferentes sistemas de coordenadas, como ficará mais claro posteriormente. Para simplificar o entendimento da notação que será adotada, os sistemas de coordenadas estão mostrados na Figura 4. Quatro sistemas de coordenadas serão utilizados: o sistema de coordenada global, $x_{i}^{(g)}$, que define os carregamentos aplicados; as coordenadas naturais do material, $x_{i}$, onde as propriedades mecânicas são definidas e $x_{1}$ está alinhado com as fibras (o ângulo entre $x_{1}$ e $x_{1}^{(g)}$ é $\alpha$ ); para definir a geometria do furo utiliza-se $x_{i}^{(h)}$, em que o ângulo entre $x_{1}^{(h)}$ e $x_{1}$ é $\beta$; e as coordenadas $x_{i}^{(l)}$, que são definidas pelo ângulo $\theta$ entre $x_{1}^{(l)}$ e $x_{1}$, são utilizadas para 
mapear a borda do furo. Antes de continuar o desenvolvimento teórico, será introduzido o seguinte operador para relacionar tensores em coordenadas diferentes (Sokolnikoff, 1956)

$$
\lambda_{i j}=\cos \left(x_{i}, x_{j}\right)
$$

Os carregamentos são transferidos de $x_{i}^{(g)}$ para $x_{i}$ como

$$
\sigma_{i j}=\lambda_{i k} \lambda_{j l} \sigma_{k l}^{(g)}
$$

Note que os carregamentos $\sigma_{i j}$ e $\sigma_{i j}^{(g)}$ são considerados como aplicados em uma região distante da placa de tal forma que os mesmos podem ser considerados como atuantes no infinito. Uma vez que as tensões são obtidas em relação aos eixos do material, se torna conveniente definir os seguintes vetores de tensão

$$
\begin{gathered}
\boldsymbol{\tau}_{1}=\left[\begin{array}{l}
\sigma_{11} \\
\sigma_{12} \\
\sigma_{13}
\end{array}\right] \\
\boldsymbol{\tau}_{2}=\left[\begin{array}{l}
\sigma_{21} \\
\sigma_{22} \\
\sigma_{23}
\end{array}\right]
\end{gathered}
$$

Assumindo o estado plano de tensões

$$
\begin{gathered}
\boldsymbol{\tau}_{1}=\left[\begin{array}{c}
\sigma_{11} \\
\sigma_{12} \\
0
\end{array}\right] \\
\boldsymbol{\tau}_{2}=\left[\begin{array}{c}
\sigma_{21} \\
\sigma_{22} \\
0
\end{array}\right]
\end{gathered}
$$

A partir dessa etapa torna-se útil separar a solução usando duas coordenadas diferentes: a do material, onde o carregamento é conhecido pela transformação anterior, e a local, que mapeia a borda do furo e define localmente as propriedades do material. A principal vantagem de usar a coordenada local é que somente a componente da tensão normal tangente ao furo não é nula, assumindo que não há pressão interna ao furo, visto que não pode haver cisalhamento e tensão normal perpendicular à superfície livre. Dessa forma, pode-se definir 


$$
\begin{aligned}
& \mathbf{Q}^{(l)}=\mathbf{Q} \cos ^{2} \theta+\left(\mathbf{R}+\mathbf{R}^{T}\right) \sin \theta \cos \theta+\mathbf{T} \sin ^{2} \theta \\
& \mathbf{R}^{(l)}=\mathbf{R} \cos ^{2} \theta+(\mathbf{T}-\mathbf{Q}) \sin \theta \cos \theta+\mathbf{R}^{T} \sin ^{2} \theta \\
& \mathbf{T}^{(l)}=\mathbf{T} \cos ^{2} \theta-\left(\mathbf{R}+\mathbf{R}^{T}\right) \sin \theta \cos \theta+\mathbf{Q} \sin ^{2} \theta
\end{aligned}
$$

E as componentes da matriz fundamental da elasticidade

$$
\begin{aligned}
& \mathbf{N}_{1}^{(l)}=-\left(\mathbf{T}^{(l)}\right)^{-1}\left(\mathbf{R}^{(l)}\right)^{T} \\
& \mathbf{N}_{2}^{(l)}=\left(\mathbf{T}^{(l)}\right)^{-1} \\
& \mathbf{N}_{3}^{(l)}=-\mathbf{R}^{(l)}\left(\mathbf{T}^{(l)}\right)^{-1}\left(\mathbf{R}^{(l)}\right)^{T}-\mathbf{Q}^{(l)}
\end{aligned}
$$

Durante o desenvolvimento do formalismo de Stroh, os tensores de Barnett-Lothe são grandezas que aparecem de forma recorrente e torna-se, então, útil explicitar os mesmos como função apenas das propriedades dos materiais. Wei e Ting (1994) publicaram as expressões desses tensores para materiais anisotrópicos gerais. A forma integral dos mesmos pode ser escrita como

$$
\begin{aligned}
& \mathbf{S}_{\mathrm{BL}}=\frac{1}{\pi} \int_{0}^{\pi} \mathbf{N}_{1}^{(1)} \mathrm{d} \theta \\
& \mathbf{H}_{\mathrm{BL}}=\frac{1}{\pi} \int_{0}^{\pi} \mathbf{N}_{2}^{(1)} \mathrm{d} \theta \\
& \mathbf{L}_{\mathrm{BL}}=\frac{1}{\pi} \int_{0}^{\pi} \mathbf{N}_{3}^{(1)} \mathrm{d} \theta
\end{aligned}
$$

Note que os tensores de Barnett-Lothe são invariantes (constantes) para o material, ou seja, independem do ponto em que está sendo analisado. Para materiais ortotrópicos, a seguinte relação explícita são obtidas para os tensores de Barnett-Lothe considerando-se tensão plana

$$
\begin{aligned}
\mathbf{S}_{\mathrm{BL}} & =\left[\begin{array}{ccc}
0 & \left(\mathrm{~S}_{\mathrm{BL}}\right)_{12} & 0 \\
\left(\mathrm{~S}_{\mathrm{BL}}\right)_{21} & 0 & 0 \\
0 & 0 & 0
\end{array}\right] \\
\mathbf{L}_{\mathrm{BL}} & =\left[\begin{array}{ccc}
\left(\mathrm{L}_{\mathrm{BL}}\right)_{11} & 0 & 0 \\
0 & \left(\mathrm{~L}_{\mathrm{BL}}\right)_{22} & 0 \\
0 & 0 & \left(\mathrm{~L}_{\mathrm{BL}}\right)_{33}
\end{array}\right]
\end{aligned}
$$


onde

$$
\begin{aligned}
& \left(S_{B L}\right)_{12}=\left[\left(\frac{E_{2}}{G_{12}}\right)+2\left(1-\sqrt{\nu_{12} \nu_{21}}\right) \sqrt{\frac{E_{2}}{E_{1}}}\right]^{-\frac{1}{2}}\left(1-\sqrt{\nu_{12} \nu_{21}}\right) \\
& \left(S_{B L}\right)_{21}=-\left\{\frac{\left[\left(\frac{E_{1}}{G_{12}}\right)+2\left(1-\sqrt{\nu_{12} \nu_{21}}\right) \sqrt{\frac{E_{1}}{E_{2}}}\right]}{\left[\left(\frac{E_{2}}{G_{12}}\right)+2\left(1-\sqrt{\nu_{12} \nu_{21}}\right) \sqrt{\frac{E_{2}}{E_{1}}}\right]}\right\}^{-\frac{1}{2}}\left[\left(\frac{E_{2}}{G_{12}}\right)+2\left(1-\sqrt{\nu_{12} \nu_{21}}\right) \sqrt{\frac{E_{2}}{E_{1}}}\right]^{-\frac{1}{2}}\left(1-\sqrt{\nu_{12} \nu_{21}}\right) \\
& \left(L_{B L}\right)_{11}=\left[\frac{E_{1}}{\left(1-\sqrt{\nu_{12} \nu_{21}}\right)}\right]\left[\left(\frac{E_{2}}{G_{12}}\right)+2\left(1-\sqrt{\nu_{12} \nu_{21}}\right) \sqrt{\frac{E_{2}}{E_{1}}}\right]^{-\frac{1}{2}}\left(1-\sqrt{\nu_{12} \nu_{21}}\right) \\
& \left(L_{B L}\right)_{22}=-\left[\frac{E_{1}}{\left.\left(1-\sqrt{\nu_{12} \nu_{21}}\right)\right]}\right]\left\{\left[\frac{\left.\left(\frac{E_{1}}{G_{12}}\right)+2\left(1-\sqrt{\nu_{12} \nu_{21}}\right) \sqrt{\frac{E_{1}}{E_{2}}}\right]}{\left[\left(\frac{E_{2}}{G_{12}}\right)+2\left(1-\sqrt{\nu_{12} \nu_{21}}\right) \sqrt{\frac{E_{2}}{E_{1}}}\right]}\right\}^{-\frac{1}{2}}\left[\left(\frac{E_{2}}{G_{12}}\right)+2\left(1-\sqrt{\nu_{12} \nu_{21}}\right) \sqrt{\frac{E_{2}}{E_{1}}}\right]^{-\frac{1}{2}}\left(1-\sqrt{\nu_{12} \nu_{21}}\right)\right. \\
& \left(L_{B L}\right)_{33}=\sqrt{G_{13} G_{23}}
\end{aligned}
$$

Para modelar tal problema central do presente estudo, faz-se útil da técnica de mapeamento conforme. A técnica de mapeamento no espaço complexo é amplamente utilizada em problemas de elasticidade para simplificar a descrição matemática da solução e uma introdução desse método pode ser encontrada em Sokolnikoff (1956). A ideia base usada é descrever a geometria da elipse como um círculo de raio unitário em um plano complexo. Nessa etapa, o desenvolvimento feito é um pouco diferente do proposto por Ting (1996) e Hwu (2009), onde o eixo da elipse coincide com o sistema de coordenadas do material, o objetivo do presente texto é apresentar uma abordagem alternativa. Para o plano real coincidente com a geometria da elipse $\left(x_{i}^{(h)}\right)$, pode-se descrever uma elipse de semieixos $r_{a}$ e $r_{b}$ como

$$
\begin{aligned}
& x_{1}^{(h)}=r_{a} \cos (\psi+\beta) \\
& x_{2}^{(h)}=r_{b} \sin (\psi+\beta)
\end{aligned}
$$


A relação entre as coordenadas do material $\left(x_{i}\right)$ e do plano que define a elipse é obtida por

$$
x_{i}=\lambda_{i j} x_{j}^{(h)}
$$

onde $\beta$ é o angulo entre $x_{1}$ e $x_{1}^{(h)}$ (ver Figura 4). Com isso, a elipse pode ser descrita em coordenadas locais como

$$
\begin{aligned}
& x_{1}=r_{a} \cos (\psi+\beta) \cos \beta-r_{b} \sin (\psi+\beta) \sin \beta \\
& x_{2}=r_{a} \cos (\psi+\beta) \sin \beta+r_{b} \sin (\psi+\beta) \cos \beta
\end{aligned}
$$

Note que $0 \leq \psi \leq 2 \pi$ para mapear a geometria do furo.

Para obter a solução $z_{\xi}=x_{1}+p_{\xi} x_{2}$ é necessário o uso da seguinte transformação

$$
z_{\xi}=c_{\xi} \zeta_{\xi}+d_{\xi} \zeta_{\xi}^{-1}
$$

onde $\zeta_{\xi}$ representa um círculo de raio unitário no espaço complexo. Basicamente, se deseja obter uma relação tal que seja possível a transformação nos espaços $z \rightarrow \zeta$, onde $|\zeta|=1$, ou seja, $\zeta=e^{i(\psi+\beta)}=\cos (\psi+\beta)+i \sin (\psi+\beta)$. Comparando as duas equações obtidas para $z_{\xi}$, pode-se escrever a seguinte relação

$$
\begin{aligned}
& r_{a}\left(\cos \beta+p_{\xi} \sin \beta\right) \cos (\psi+\beta)-r_{b}\left(\sin \beta+p_{\xi} \cos \beta\right) \sin (\psi+\beta)= \\
& \left(c_{\xi}+d_{\xi}\right) \cos (\psi+\beta)+i\left(c_{\xi}-d_{\xi}\right) \sin (\psi+\beta)
\end{aligned}
$$

$\log 0$

$$
\begin{aligned}
c_{\xi} & =\frac{r_{a}\left(\cos \beta+p_{\xi} \sin \beta\right)-i r_{b}\left(\sin \beta+p_{\xi} \cos \beta\right)}{2} \\
d_{\xi} & =\frac{r_{a}\left(\cos \beta+p_{\xi} \sin \beta\right)+i r_{b}\left(\sin \beta+p_{\xi} \cos \beta\right)}{2}
\end{aligned}
$$

Uma vez que a transformação no espaço complexo é obtida e a condição de contorno pode ser descrita, convém também escrever as soluções dos campos de deslocamentos e das funções de tensões, obtidas anteriormente de forma genérica, também no espaço complexo

$$
\begin{aligned}
& \mathbf{u}=2 \operatorname{Re}[\mathbf{V f}(\zeta)] \\
& \boldsymbol{\phi}=2 \operatorname{Re}[\mathbf{W} \mathbf{f}(\zeta)]
\end{aligned}
$$


Para resolver o problema da distribuição das tensões, as seguintes condições de contorno precisam ser satisfeitas

$$
\begin{aligned}
& \left.\phi\right|_{\infty} \rightarrow \phi^{\infty} \\
& \left.\phi\right|_{\Gamma}=0
\end{aligned}
$$

onde $\Gamma$ representa a região da borda do furo e $\infty$ uma região distante da mesma.

Para a solução do problema, supõe-se a seguinte solução da função de tensão

$$
\phi=\phi^{\infty}+2 \operatorname{Re}\left\{\mathbf{W}\left\langle\zeta_{\xi}^{-1}\right\rangle \mathbf{q}\right\}
$$

Note que para uma distância muito grande do furo $(\infty), \zeta_{\xi}^{-1} \rightarrow 0$ e na borda do furo

$$
\begin{aligned}
& \left.\phi\right|_{\Gamma}=\left(r_{a} \cos (\psi+\beta) \cos \beta-r_{b} \sin (\psi+\beta) \sin \beta\right) \boldsymbol{\tau}_{2}- \\
& \left(r_{a} \cos (\psi+\beta) \sin \beta+r_{b} \sin (\psi+\beta) \cos \beta\right) \boldsymbol{\tau}_{1}+2 \operatorname{Re}\left\{e^{-i \psi} \mathbf{W} \mathbf{q}\right\}
\end{aligned}
$$

Usando as seguintes relações

$$
\begin{aligned}
& e^{-i(\psi+\beta)}\left(r_{a} \cos \beta-i r_{b} \sin \beta\right) \boldsymbol{\tau}_{2}=\left[\begin{array}{l}
\left(r_{a} \cos (\psi+\beta) \cos \beta-i r_{a} \sin (\psi+\beta) \cos \beta\right)- \\
\left(i r_{b} \cos (\psi+\beta) \sin \beta+r_{b} \sin (\psi+\beta) \sin \beta\right)
\end{array}\right] \boldsymbol{\tau}_{2} \\
& -e^{-i(\psi+\beta)}(a \sin \beta+i b \cos \beta) \tau_{1}=-\left[\begin{array}{l}
\left(r_{a} \cos (\psi+\beta) \sin \beta-i r_{a} \sin (\psi+\beta) \sin \beta\right)+ \\
\left(i r_{b} \cos (\psi+\beta) \cos \beta+r_{b} \sin (\psi+\beta) \cos \beta\right)
\end{array}\right] \boldsymbol{\tau}_{1}
\end{aligned}
$$

a equação (51) pode ser, então, manipulada e reescrita como

$$
\left.\boldsymbol{\phi}\right|_{\Gamma}=\operatorname{Re}\left\{e^{-i(\psi+\beta)}\left(2 \mathbf{W q}+\left(r_{a} \cos \beta-i r_{b} \sin \beta\right) \boldsymbol{\tau}_{2}-\left(r_{a} \sin \beta+i r_{b} \cos \beta\right) \boldsymbol{\tau}_{1}\right)\right\}
$$

E então usando a condição de contorno $\left.\phi\right|_{\Gamma}=0$, o vetor $\mathbf{q}$ pode ser escrito como

$$
\mathbf{q}=-\frac{1}{2} \mathbf{W}^{-1}\left(\left(r_{a} \cos \beta-i r_{b} \sin \beta\right) \boldsymbol{\tau}_{2}-\left(r_{a} \sin \beta+i r_{b} \cos \beta\right) \boldsymbol{\tau}_{1}\right)
$$

Com esse resultado, a solução geral da função de tensão de uma placa infinita anisotrópica com furo elítico submetida a um carregamento genérico no plano (no infinito) pode ser, então, escrita como

$$
\boldsymbol{\phi}=x_{1} \boldsymbol{\tau}_{2}-x_{2} \boldsymbol{\tau}_{1}-\operatorname{Re}\left\{\left(\mathbf{W}\left\langle\zeta_{\xi}^{-1}\right\rangle \mathbf{W}^{-1}\right)\left(\left(r_{a} \cos \beta-i r_{b} \sin \beta\right) \boldsymbol{\tau}_{2}-\left(r_{a} \sin \beta+i r_{b} \cos \beta\right) \boldsymbol{\tau}_{1}\right)\right\}
$$

Na borda do furo, a única componente de tensão atuante é a tensão normal na direção tangente à borda, que pode ser obtida pelo seguinte produto escalar

$$
\sigma_{11}^{(l)}=-\left(\mathbf{e}_{1}^{(l)}\right)^{T} \frac{\partial \phi}{\partial \mathbf{e}_{2}^{(l)}}
$$

onde 
$\left(\mathbf{e}_{1}^{(l)}\right)^{T}=\left(\begin{array}{lll}\cos \theta & \sin \theta & 0\end{array}\right)$

$\left(\mathbf{e}_{2}^{(l)}\right)^{T}=\left(\begin{array}{lll}-\sin \theta & \cos \theta & 0\end{array}\right)$

e a derivada pode ser expressa por

$$
\begin{aligned}
& \frac{\partial \phi}{\partial \mathbf{e}_{2}^{(l)}}=\frac{\partial x_{1}}{\partial \mathbf{e}_{2}^{(l)}} \boldsymbol{\tau}_{2}-\frac{\partial x_{2}}{\partial \mathbf{e}_{2}^{(l)}} \boldsymbol{\tau}_{1}- \\
& \operatorname{Re}\left\{\left(\mathbf{W}\left\langle\frac{\partial\left(\zeta_{\xi}^{-1}\right)}{\partial \mathbf{e}_{2}^{(l)}}\right\rangle \mathbf{W}^{-1}\right)\left(\left(r_{a} \cos \beta-i r_{b} \sin \beta\right) \boldsymbol{\tau}_{2}-\left(r_{a} \sin \beta+i r_{b} \cos \beta\right) \boldsymbol{\tau}_{1}\right)\right\}
\end{aligned}
$$

Para calcularmos a última derivada mostrada na equação (58) é necessário obter uma relação entre $\theta$ e $\psi$. Supondo que a distância entre o centro do sistema de coordenadas e um ponto qualquer da elipse seja $\rho$, o comprimento de um arco infinitesimal

$$
d s=\rho d \psi=\left(d x_{1}^{2}+d x_{2}^{2}\right)^{\frac{1}{2}}
$$

Usando as equações (44.a,b)

$$
\begin{aligned}
& d x_{1}=-\left(\left(r_{a} \cos \beta\right) \sin (\psi+\beta)+\left(r_{b} \sin \beta\right) \cos (\psi+\beta)\right) d \psi \\
& d x_{2}=\left(-\left(r_{a} \sin \beta\right) \sin (\psi+\beta)+\left(r_{b} \cos \beta\right) \cos (\psi+\beta)\right) d \psi
\end{aligned}
$$

e então

$$
\rho^{2}=\left(r_{a}^{2} \sin ^{2}(\psi+\beta)+r_{b}^{2} \cos ^{2}(\psi+\beta)\right)
$$

pelo triângulo de lados infinitesimais,

$$
\begin{aligned}
& (\rho d \psi) \cos \theta=d x_{1} \\
& (\rho d \psi) \sin \theta=d x_{2}
\end{aligned}
$$

e então

$$
\begin{aligned}
& \rho \cos \theta=-\left(\left(r_{a} \cos \beta\right) \sin (\psi+\beta)+\left(r_{b} \sin \beta\right) \cos (\psi+\beta)\right) \\
& \rho \sin \theta=\left(-\left(r_{a} \sin \beta\right) \sin (\psi+\beta)+\left(r_{b} \cos \beta\right) \cos (\psi+\beta)\right)
\end{aligned}
$$

Reorganizando as equações, pode-se obter

$$
\begin{aligned}
& \sin (\psi+\beta)=-\frac{\rho}{r_{a}} \cos (\theta-\beta) \\
& \cos (\psi+\beta)=\frac{\rho}{r_{b}} \sin (\theta-\beta)
\end{aligned}
$$


Agora pode-se então aplicar a regra da cadeia,

$$
\frac{\partial\left(\zeta_{\xi}^{-1}\right)}{\partial \mathbf{e}_{2}^{(l)}}=\frac{\partial\left(\zeta_{\xi}^{-1}\right)}{\partial \zeta_{\xi}} \frac{\partial \zeta_{\xi}}{\partial \psi} \frac{\partial \psi}{\partial z_{\xi}}\left[\frac{\partial z_{\xi}}{\partial x_{1}} \frac{\partial x_{1}}{\partial \mathbf{e}_{2}^{(l)}}+\frac{\partial z_{\xi}}{\partial x_{2}} \frac{\partial x_{2}}{\partial \mathbf{e}_{2}^{(l)}}\right]
$$

e resolvendo cada parcela separadamente

$$
\begin{aligned}
& \frac{\partial\left(\zeta_{\xi}^{-1}\right)}{\partial \zeta_{\xi}}=-\zeta_{\xi}^{-2}=-e^{-2(\psi+\beta) i} \\
& \frac{\partial \zeta_{\xi}}{\partial \psi}=\frac{\partial\left(e^{i(\psi+\beta)}\right)}{\partial \psi}=i e^{(\psi+\beta) i} \\
& \frac{\partial z_{\xi}}{\partial \psi}=-\left(r_{a}\left(\cos \beta+p_{\xi} \sin \beta\right) \sin (\psi+\beta)+r_{b}\left(\sin \beta-p_{\xi} \cos \beta\right) \cos (\psi+\beta)\right) \\
& \frac{\partial z_{\xi}}{\partial x_{1}}=1 \\
& \frac{\partial x_{1}}{\partial \mathbf{e}_{2}^{(l)}}=-\sin \theta \\
& \frac{\partial z_{\xi}}{\partial x_{2}}=p_{\xi} \\
& \frac{\partial x_{2}}{\partial \mathbf{e}_{2}^{(l)}}=\cos \theta \\
& \text { e aplicando } \\
& \frac{\partial\left(\zeta_{\xi}^{-1}\right)}{\partial \mathbf{e}_{2}^{(l)}}=\left(-\frac{i e^{-i(\psi+\beta)}}{\rho}\right)\left(p_{\xi}^{(l)}\right)
\end{aligned}
$$

Onde são os autovalores em coordenadas locais. Um resultado interessante sobre os autovalores em coordenadas locais é que o valor médio dos mesmos, independente do material, no domínio, ou seja, $0^{\circ} \leq \theta \leq 180^{\circ}$, é $\pm i$ que são os autovalores dos materiais isotrópicos (Hwu, 2009). Substituindo esse resultado na equação (58)

$$
\begin{aligned}
& \frac{\partial \phi}{\partial \mathbf{e}_{2}^{(l)}}=-\boldsymbol{\tau}_{2} \sin \theta-\boldsymbol{\tau}_{1} \cos \theta+ \\
& \frac{1}{\rho} \operatorname{Re}\left\{i e^{-i(\psi+\beta)}\left(\mathbf{W}\left\langle p_{\xi}^{(l)}\right\rangle \mathbf{W}^{-1}\right)\left(\left(r_{a} \cos \beta-i r_{b} \sin \beta\right) \boldsymbol{\tau}_{2}-\left(r_{a} \sin \beta+i r_{b} \cos \beta\right) \boldsymbol{\tau}_{1}\right)\right\}
\end{aligned}
$$

Torna-se, então, vantajoso o uso da seguinte relação, que por simplicidade não será deduzida e pode ser facilmente obtida em Hwu (2009), 


$$
\mathbf{W}\left\langle p_{\xi}^{(l)}\right\rangle \mathbf{W}^{-1}=\mathbf{G}_{1}^{(l)}+i \mathbf{G}_{3}^{(l)}
$$

onde

$$
\begin{aligned}
& \mathbf{G}_{1}^{(l)}=\left[\mathbf{N}_{1}^{(l)}\right]^{T}-\mathbf{N}_{3}^{(l)} \mathbf{S} \mathbf{L}^{-1} \\
& \mathbf{G}_{3}^{(l)}=-\mathbf{N}_{3}^{(l)} \mathbf{L}^{-1}
\end{aligned}
$$

Note que as matrizes $\mathbf{N}_{1}^{(l)}$ e $\mathbf{N}_{3}^{(l)}$ são definidas em coordenadas locais e as matrizes $\mathbf{S}$ e L são constantes para o material (independem do ponto em que se esteja analisando), e que a vantagem de utilizar a expressão (69) é que não se torna necessário calcular os autovalores do material, diminuindo esforço computacional.

Utilizando essa identidade, a derivada da função de tensão pode ser então reescrita como

$$
\begin{aligned}
& \frac{\partial \phi}{\partial \mathbf{e}_{2}^{(l)}}=-\boldsymbol{\tau}_{2} \sin \theta-\boldsymbol{\tau}_{1} \cos \theta+ \\
& \operatorname{Re}\left\{\left(i \sin (\theta-\beta)-\frac{r_{b}}{r_{a}} \cos (\theta-\beta)\right)\left(\mathbf{G}_{1}^{(l)}+i \mathbf{G}_{3}^{(l)}\right)\left(\left(\frac{r_{a}}{r_{b}} \cos \beta-i \sin \beta\right) \boldsymbol{\tau}_{2}-\left(\frac{r_{a}}{r_{b}} \sin \beta+i \cos \beta\right) \boldsymbol{\tau}_{1}\right)\right\}
\end{aligned}
$$

Por último, juntando os resultados expressos na equação (71) e substituindo na equação (56), a tensão pode ser então calculada. Para evitar equações excessivamente longas, a equação explicita de $\sigma_{11}^{(l)}$ será omitida, todavia, todas as parcelas são conhecidas e podem ser facilmente implementadas. Pode-se perceber que quando $\beta=0^{\circ}$, o resultado coincide com a abordagem mostrada por Hwu (2009).

Hwu e Ting (1989) foram os primeiros a resolverem o problema de placas anisotrópicas com furos ou inclusões elípticas com carregamentos no plano usando o formalismo de Stroh. Duas conclusões especialmente interessantes foram tiradas pelos autores para o caso de um furo elíptico em material anisotrópico sujeito a tensão uniaxial perpendicularmente ao maior semi-eixo da elipse: a concentração de tensão em um ponto sobre o menor semi-eixo da elipse independe do formato da mesma, isto é, da relação $r_{a} / r_{b}$, e a concentração de tensão no maior semi-eixo da elipse apresenta uma forma simples; mas vale ressaltar que esse ponto não é necessariamente o de maior tensão, como acontece com materiais isotrópicos, resultando que, apesar da simplicidade evidente da solução para esse ponto, a equação precisa ser avaliada ao longo de todo o domínio para ter sua criticidade 
avaliada de forma correta. Muitos outros autores, como será mostrado posteriormente, principalmente na busca de obter uma solução aproximada para o caso de placas finitas, consideram apenas a expressão simplificada da concentração de tensão no ponto sobre o maior semi-eixo da elipse e não comentam em seus trabalhos que a tensão máxima pode estar atuando em outro ponto para o caso geral, o que acaba resultando em um erro conceitual que limita a utilização do modelo proposto. Para furos genéricos Hwu (1990, 1992) obteve a solução aproximada da distribuição de tensões ao redor de furos com diversos formatos em placas anisotrópicas utilizando o formalismo de Stroh e avaliou a validade das soluções.

\subsection{Teoria Clássica dos Laminados (TCL)}

$\mathrm{Na}$ prática, os carregamentos geralmente são multiaxiais, e a aplicação de um laminado com todas as lâminas na mesma direção não se torna viável porque oferece a resistência apenas em uma direção. Levando isso em consideração, torna-se útil a aplicação de laminados, que pode ser definido como um conjunto de lâminas sobrepostas.

Como base da TCL, as seguintes hipóteses serão consideradas (Christensen, 2005):

- considera-se que as lâminas são perfeitamente coladas, não podendo então haver separação, e que cada lâmina é ortotrópica e pode ter uma orientação qualquer;

- a espessura da placa é muito menor do que as outras dimensões da mesma;

- a análise está restrita a pequenos deslocamentos;

- deformações cisalhantes agindo em planos perpendiculares às lâminas podem ser desprezadas (estado plano de tensões);

- os deslocamentos no plano são funções lineares da coordenada que define a espessura da placa (hipótese de Kirchhoff-Love);

- a espessura da placa é constante;

- deformação normal na direção da $x_{3}$ é suposta constante ao longo da espessura.

Seguindo as hipóteses mencionadas, o campo de deslocamentos em coordenadas globais pode ser escrito como 


$$
\begin{aligned}
& \tilde{u}_{1}^{(g)}=\left.\tilde{u}_{1}^{(g)}\right|_{0}\left(x_{1}^{(g)}, x_{2}^{(g)}\right)+x_{3}^{(g)} \tilde{f}_{1}\left(x_{1}^{(g)}, x_{2}^{(g)}\right) \\
& \tilde{u}_{2}^{(g)}=\left.\tilde{u}_{2}^{(g)}\right|_{0}\left(x_{1}^{(g)}, x_{2}^{(g)}\right)+x_{3}^{(g)} \tilde{f}_{2}\left(x_{1}^{(g)}, x_{2}^{(g)}\right) \\
& \tilde{u}_{3}^{(g)}=\left.\tilde{u}_{3}^{(g)}\right|_{0}\left(x_{1}^{(g)}, x_{2}^{(g)}\right)+x_{3}^{(g)} \tilde{f}_{3}\left(x_{1}^{(g)}, x_{2}^{(g)}\right)
\end{aligned}
$$

onde $\left.*\right|_{0}$ se refere ao valor da grandeza quando $x_{3}^{(g)}=0$, e.g. $\left.\tilde{u}_{1}^{(g)}\right|_{0}=\tilde{u}_{1}^{(g)}\left(x_{1}^{(g)}, x_{2}^{(g)}, 0\right)$, e o símbolo $\tilde{*}^{*}$ será utilizado todas as vezes que as grandezas físicas forem referentes ao laminado e não à lâmina, dessa forma ficará claro a diferenciação dos mesmos. Como está sendo considerado o estado plano de tensões,

$$
\begin{aligned}
& \tilde{\varepsilon}_{13}^{(g)}=\frac{1}{2}\left(\frac{\partial \tilde{u}_{1}^{(g)}}{\partial x_{3}^{(g)}}+\frac{\partial \tilde{u}_{3}^{(g)}}{\partial x_{1}^{(g)}}\right)=\frac{1}{2}\left(\tilde{f}_{1}\left(x_{1}, x_{2}\right)+\frac{\partial \tilde{u}_{3}^{(g)}}{\partial x_{1}^{(g)}}\right)=0 \\
& \tilde{\varepsilon}_{23}^{(g)}=\frac{1}{2}\left(\frac{\partial \tilde{u}_{2}^{(g)}}{\partial x_{3}^{(g)}}+\frac{\partial \tilde{u}_{3}^{(g)}}{\partial x_{2}^{(g)}}\right)=\frac{1}{2}\left(\tilde{f}_{2}\left(x_{1}, x_{2}\right)+\frac{\partial \tilde{u}_{3}^{(g)}}{\partial x_{2}^{(g)}}\right)=0
\end{aligned}
$$

o que resulta em

$$
\begin{aligned}
& \tilde{f}_{1}\left(x_{1}, x_{2}\right)=-\frac{\partial \tilde{u}_{3}^{(g)}}{\partial x_{1}^{(g)}} \\
& \tilde{f}_{2}\left(x_{1}, x_{2}\right)=-\frac{\partial \tilde{u}_{3}^{(g)}}{\partial x_{2}^{(g)}}
\end{aligned}
$$

Com esses resultados, as deformações no plano podem ser escritas como

$$
\begin{aligned}
& \tilde{\varepsilon}_{11}^{(g)}=\frac{\partial \tilde{u}_{1}^{(g)}}{\partial x_{1}^{(g)}}=\left.\tilde{\varepsilon}_{11}^{(g)}\right|_{0}-x_{3}^{(g)} \tilde{\kappa}_{11}^{(g)} \\
& \tilde{\varepsilon}_{22}^{(g)}=\frac{\partial \tilde{u}_{2}^{(g)}}{\partial x_{2}^{(g)}}=\left.\tilde{\varepsilon}_{22}^{(g)}\right|_{0}-x_{3}^{(g)} \tilde{\kappa}_{22}^{(g)} \\
& \tilde{\varepsilon}_{12}^{(g)}=\frac{1}{2}\left(\frac{\partial \tilde{u}_{1}^{(g)}}{\partial x_{2}^{(g)}}+\frac{\partial \tilde{u}_{2}^{(g)}}{\partial x_{1}^{(g)}}\right)=\left.\tilde{\varepsilon}_{12}^{(g)}\right|_{0}-x_{3}^{(g)} \tilde{\kappa}_{12}^{(g)}
\end{aligned}
$$

onde $\tilde{\kappa}_{i j}$ é a curvatura associada à flexão se $i=j\left(\tilde{\kappa}_{i i}=\partial^{2} \tilde{u}_{3}^{(g)} / \partial\left(x_{i}^{(g)}\right)^{2}\right)$ e à torção se $i \neq j\left(\tilde{\kappa}_{i j}=\partial^{2} \tilde{u}_{3}^{(g)} / \partial x_{i}^{(g)} \partial x_{j}^{(g)}\right)$. O tensor das deformações pode, então, ser escrito como 
$\tilde{\varepsilon}_{i j}^{(g)}=\left.\tilde{\varepsilon}_{i j}^{(g)}\right|_{0}-x_{3}^{(g)} \tilde{\kappa}_{i j}^{(g)}$

e a relação constitutiva de cada lâmina como

$\left[\sigma_{i j}^{(g)}\right]_{m}=\left[s_{i j k l}^{(g)}\right]_{m}\left[\varepsilon_{k l}^{(g)}\right]_{m}$

onde $\left[\sigma_{i j}^{(g)}\right]_{m},\left[s_{i j k l}^{(g)}\right]_{m}$ e $\left[\varepsilon_{k l}^{(g)}\right]_{m}$ são os tensores das tensões, deformações e rigidez da $m$ - th lâmina em coordenada global. Pela condição de compatibilidade, a deformação precisa ser contínua, ou seja, $\tilde{\varepsilon}_{i j}^{(g)}=\varepsilon_{i j}^{(g)}$ e $\tilde{\kappa}_{i j}^{(g)}=\kappa_{i j}^{(g)}$.

As forças e os momentos por unidade de comprimento são

$$
\begin{aligned}
& F_{i j}^{(g)}=\sum_{m=1}^{n}\left\{\int_{\left(x_{3}^{(g)}\right)_{m-1}}^{\left(x_{3}^{(g)}\right)_{m}}\left(\left[s_{i j k l}^{(g)}\right]_{m}\left[\left.\varepsilon_{k l}^{(g)}\right|_{0}+x_{3}^{(g)} \kappa_{k l}^{(g)}\right]\right) d x_{3}^{(g)}\right\} \\
& M_{i j}^{(g)}=\sum_{m=1}^{n}\left\{\int_{\left(x_{3}^{(g)}\right)_{m-1}}^{\left(x_{3}^{(g)}\right)_{m}}\left(\left[s_{i j k l}^{(g)}\right]_{m}\left[\left.\varepsilon_{k l}^{(g)}\right|_{0}+x_{3}^{(g)} \kappa_{k l}^{(g)}\right]\right) x_{3}^{(g)} d x_{3}^{(g)}\right\}
\end{aligned}
$$

Torna-se, então, útil definir os seguintes tensores

$$
\begin{aligned}
& A_{i j k l}^{(g)}=\sum_{m=1}^{n}\left\{\int_{\left(x_{3}^{(g)}\right)_{m-1}}^{\left(x_{3}^{(g)}\right)_{m}}\left[s_{i j k l}^{(g)}\right]_{m} d x_{3}^{(g)}\right\}=\sum_{m=1}^{n}\left\{\left[s_{i j k l}^{(g)}\right]_{m}\left(\left[x_{3}^{(g)}\right]_{m}-\left[x_{3}^{(g)}\right]_{m-1}\right)\right\} \\
& B_{i j k l}^{(g)}=\sum_{m=1}^{n}\left\{\int_{\left(x_{3}^{(g)}\right)_{m-1}}^{\left(x_{3}^{(g)}\right)_{m}}\left[s_{i j k l}^{(g)}\right]_{m} x_{3}^{(g)} d x_{3}^{(g)}\right\}=\frac{1}{2} \sum_{m=1}^{n}\left\{\left[s_{i j k l}^{(g)}\right]_{m}\left(\left[\left(x_{3}^{(g)}\right)^{2}\right]_{m}-\left[\left(x_{3}^{(g)}\right)^{2}\right]_{m-1}\right)\right\} \\
& D_{i j k l}^{(g)}=\sum_{m=1}^{n}\left\{\int_{\left(x_{3}^{(g)}\right)_{m-1}}^{\left(x_{3}^{(g)}\right)_{m}}\left[s_{i j k l}^{(g)}\right]_{m}\left(x_{3}^{(g)}\right)^{2} d x_{3}^{(g)}\right\}=\frac{1}{3} \sum_{m=1}^{n}\left\{\left[s_{i j k l}^{(g)}\right]_{m}\left(\left[\left(x_{3}^{(g)}\right)^{3}\right]_{m}-\left[\left(x_{3}^{(g)}\right)^{3}\right]_{m-1}\right)\right\}
\end{aligned}
$$

E então a seguinte relação matricial pode ser obtida

$$
\left[\begin{array}{c}
\boldsymbol{F} \\
\boldsymbol{M}
\end{array}\right]=\left[\begin{array}{ll}
\boldsymbol{A} & \boldsymbol{B} \\
\boldsymbol{B} & \boldsymbol{D}
\end{array}\right]\left[\begin{array}{l}
\varepsilon^{0} \\
\boldsymbol{\kappa}
\end{array}\right]
$$

Com essa relação se torna evidente o acoplamento entre o momento aplicado e a deformação normal no plano médio, assim como a força e a curvatura para o caso genérico. Entretanto, o presente trabalho visa analisar apenas laminados simétricos e com essa restrição, $\boldsymbol{B}=\mathbf{0}$, ou seja, não há o acoplamento entre esses efeitos. 
Considerando apenas os carregamentos de forças distribuídas não nulos e o laminado simétrico,

$$
\left[\varepsilon_{i j}^{(g)}\right]_{m}=\left(A_{i j k l}^{(g)}\right)^{-1} N_{i j}^{(g)}
$$

Assumindo que o conjunto de lâminas ortotrópicas resulta em um laminado ortotrópico e o mesmo pode ser considerado como uma única camada homogênea com rigidez equivalente para se obter o campo de deformações (Vasiliev e Morozov, 2001), a matriz de flexibilidade equivalente pode ser obtida por

$$
\tilde{\boldsymbol{c}}^{(g)}=t\left(\boldsymbol{A}^{(g)}\right)^{-1}
$$

Para se obter as tensões atuantes em cada lâmina deve-se primeiro calcular as deformações obtidas pelo laminado de rigidez equivalente e depois aplicar a relação constitutiva da lâmina que se deseja estudar; utilizar as propriedades equivalentes para calcular as tensões é um erro conceitual. Um exemplo claro dessa ideia é a associação de molas em paralelo para obter a rigidez equivalente do sistema; para o caso de molas, podese obter informações sobre os deslocamentos do sistema equivalente, mas não se pode utilizar diretamente a força total que está atuando na estrutura para estudar o que acontece em cada mola, é necessário fazer a divisão da força e avaliar a parcela da força que atua em cada mola.

A vantagem de calcular as propriedades equivalentes do laminado é que o desenvolvimento do formalismo de Stroh pode ser diretamente aplicável para calcular as deformações.

É sabido que quando camadas consecutivas possuem propriedades diferentes, uma tensão interlaminar é intrinsicamente resultante devido à diferença entre as propriedades mecânicas de acordo com a direção (Kant e Swaminathan, 2000), o que influencia principalmente em problemas de delaminação. Todavia, esse fenômeno não será considerado no presente estudo. 


\section{Critérios de falha para laminados unidirecionais}

$\mathrm{Na}$ presente seção serão mostrados critérios de falha baseados em condições de carregamento estático. Será considerada como falha da estrutura o momento em que a primeira lâmina falhar (FPF - First Ply Failure); o dano progressivo não é objetivo deste trabalho.

Na década de 90 deu-se início um desafio global com o objetivo de avaliar qual dos presentes modelos existentes melhor descreveria a falha em materiais compósitos poliméricos com fibras unidirecionais. De acordo com Hinton et al. (2004), mesmo com diversas teorias propostas, $90 \%$ dos projetistas de materiais compósitos usam as clássicas teorias da máxima tensão, da máxima deformação ou algum ajuste polinomial. De modo a testar cada teoria, foram apresentados dados experimentais de propriedades das fibras, das matrizes e das lâminas como valores de entrada para as estimativas dos modelos. Para verificar a aplicação, alguns casos importantes de carregamentos e arranjo das lâminas nos laminados foram também escolhidos e fornecidos aos autores.

Uma vez que os dados básicos foram disponibilizados, cada autor estimou características como o envelope de falha e a curva tensão deformação do laminado de acordo com o carregamento e foram publicados. Posteriormente, os organizadores divulgaram os resultados experimentais e compararam as estimativas e depois, então, os autores tiverem uma nova oportunidade de melhorar a sua previsão com os resultados experimentais previstos e justificar os ajustes do modelo. Por último, os organizadores fizeram um artigo com as recomendações finais e as conclusões do exercício.

Essa competição foi denominada como World Wide Failure Exercise (WWFE) e está em sua terceira edição. Como o WWFE-III ainda não está concluído, apenas os resultados do WWFE-I e WWFE-II serão utilizados como base.

De acordo com Soden et al. (2004), os seguintes modelos obtiveram uma melhor predição dos resultados no WWFE-I: Tsai-Wu, Puck and Cuntze (na maioria das vezes os modelos serão chamados de acordo com o nome do principal pesquisador). Desses modelos, a abordagem de Cuntze é a única que não será estudada a seguir, porque, de acordo com o próprio autor (2004), para análises de danos iniciais (FPF), os resultados são similares à abordagem proposta por Puck (que será detalhadamente estudada 
posteriormente), a principal diferença entre elas é a maneira em que a progressão do dano é avaliada. Kaddour e Hinton (2013) apontaram o modelo proposto por Carrere e LaRC05 como os melhores do WWFE-II, incluindo os dois em posições de destaque. A abordagem de Carrere inclui aspectos da micromecânica, o que não está no escopo do presente trabalho, dessa forma, o mesmo não será detalhado. Para uma comparação entre os principais resultados do WWFE, os envelopes de falha estão apresentados no Anexo II.

Os modelos fenomenológicos não são tão populares quanto o de Tsai-Wu por serem mais trabalhosos para implementar. A questão de ser mais trabalhoso é justificada pelo fato de que são diferenciados tipos de falha da fibra e da matriz, resultando então em mecanismos de falha concorrentes que precisam ser verificados, enquanto no modelo de Tsai-Wu tem-se apenas uma equação. Todavia, o acréscimo de trabalho é justificado por um maior entendimento físico da falha em materiais compósitos, mas, consequentemente, restringe o modelo ao caso de laminados unidirecionais. Vale lembrar que como Tsai-Wu usa um ajuste polinomial genérico, pode-se aplicá-lo de forma geral a qualquer material anisotrópico.

Tsai e Melo (2014) propuseram uma nova metodologia para projetar compósitos de fibra de carbono. Eles consideraram o traço da matriz de rigidez como sendo invariante para as lâminas com fibras de carbono, o que resulta na diminuição do número de testes necessários para a caracterização do material, e de acordo com os autores, pode-se considerar a posição das fibras como se estivessem variando de uma forma genérica e, mesmo na posição que resulte em maior dano considerando a abordagem de FPF, o nível de deformações é maior do que usualmente admissível na indústria aeroespacial e então a orientação das fibras pode ser definida baseada apenas em requisitos adicionais da estrutura, como rigidez. Essa metodologia tem grande aplicação prática principalmente pelo fato de que o processo de otimização de laminados inclui uma grande gama de variáveis. Esse método não será utilizado no presente estudo porquê: i) não há uma estrutura específica a ser projetada; ii) em casos de concentradores de tensões, os níveis de deformações da pior posição possível das fibras resultaria em um superdimensionamento estrutural.

Para todos os modelos que serão apresentados, a falha é considerada quando a função proposta é igual a um. Inicialmente, os modelos serão apresentados em sua forma 
tridimensional genérica e posteriormente o caso bidimensional (estado plano de tensões) será estudado de forma mais detalhada.

\subsection{Critério de Tsai-Wu}

\subsubsection{Caso Geral}

Tsai e Wu (1971) propuseram um critério de falha genérico para materiais anisotrópicos. A ideia central dos autores não era ter a discriminação exata do tipo de falha, tendo em vista os vários mecanismos possíveis de falha, especialmente para materiais compósitos (ruptura e micro-flambagem das fibras, cavitação e propagação de trincas na matriz e delaminação, por exemplo), mas sim obter uma função de fácil implementação e utilização.

Segundo os mesmos, era consenso entre os pesquisadores e projetistas a forma de estimar falha para materiais submetidos a um estado uniaxial de tensão em relação ao sistema de coordenada utilizado para definir as propriedades mecânicas, como a resistência. Todavia, o que diferenciava os critérios de falha era a forma utilizada para quantificar o efeito de iterações em estados de tensões multiaxias.

Usando notação indicial, a seguinte função pode ser definida

$$
f_{T W}=O_{i j} \sigma_{i j}+O_{i j k l}^{\prime} \sigma_{i j} \sigma_{k l}
$$

onde $f_{T W}$ é a função polinomial utilizada para descrever um estado de tensão, $O_{i j}$ são os termos que representam o efeito linear de cada carregamento, inclusive se são positivos ou negativos, e $O_{i j k l}^{\prime}$ são os termos que representam o efeito não-linear dos carregamentos, assim como a interação das tensões em estados multiaxiais. A falha ocorrerá se

$$
f_{T W}=1
$$

Uma das vantagens citadas pelos autores é que, uma vez que as funções que representam as resistências $\left(O_{i j}\right.$ e $O_{i j k l}^{\prime}$ ) são tensores, as mesmas podem ser rotacionadas e pode-se, então, obtê-las nas direções em que o carregamento está sendo aplicado. O que difere de outros critérios similares é que na maioria deles necessariamente as tensões 
precisam ser transformadas, enquanto no critério de Tsai-Wu pode-se escolher entre rotacionar as tensões ou as resistências.

Uma forma alternativa de utilizar o critério de Tsai-Wu é escrevê-lo em função das deformações, ou seja

$$
f_{T W}=Y_{i j} \varepsilon_{i j}+Y_{i j k l}^{\prime} \varepsilon_{i j} \varepsilon_{k l}
$$

onde $Y_{i j}$ e $Y_{i j k l}^{\prime}$ têm funções similares a $O_{i j}$ e $O_{i j k l}^{\prime}$.

Para um material tridimensional que seja caracterizado por uma anisotropia de forma mais genérica possível são necessárias 27 constantes independentes para se caracterizar o modelo. Entretanto, se for considerado o material ortotrópico, a equação (83) pode ser reescrita de forma simplificada como

$$
\begin{aligned}
& f_{T W}=a_{11} \sigma_{11}+a_{22} \sigma_{22}+a_{33} \sigma_{33}+a_{12} \sigma_{12}+a_{13} \sigma_{13}+a_{23} \sigma_{23}+ \\
& a_{1111} \sigma_{11}^{2}+a_{2222} \sigma_{22}^{2}+a_{3333} \sigma_{33}^{2}+a_{1212} \sigma_{12}^{2}+a_{1313} \sigma_{13}^{2}+a_{2323} \sigma_{23}^{2}+ \\
& a_{1122} \sigma_{11} \sigma_{22}+a_{1133} \sigma_{11} \sigma_{33}+a_{2233} \sigma_{22} \sigma_{33}+a_{1112} \sigma_{11} \sigma_{12}+a_{1113} \sigma_{11} \sigma_{13}+a_{1123} \sigma_{11} \sigma_{23}+ \\
& a_{2212} \sigma_{22} \sigma_{12}+a_{2213} \sigma_{22} \sigma_{13}+a_{2223} \sigma_{22} \sigma_{23}+a_{3312} \sigma_{33} \sigma_{12}+a_{3313} \sigma_{33} \sigma_{13}+a_{3323} \sigma_{33} \sigma_{23}
\end{aligned}
$$

Considerando que os laminados são transversalmente isotrópicos, pode-se reorganizar a expressão anterior para se obter

$$
\begin{aligned}
& f_{T W}=a_{11} \sigma_{11}+a_{22}\left(\sigma_{22}+\sigma_{33}\right)+a_{12}\left(\sigma_{12}+\sigma_{13}\right)+a_{23} \sigma_{23}+a_{1111} \sigma_{11}^{2}+a_{2222}\left(\sigma_{22}^{2}+\sigma_{33}^{2}\right)+ \\
& a_{1212}\left(\sigma_{12}^{2}+\sigma_{13}^{2}\right)+a_{2323} \sigma_{23}^{2}+a_{1122} \sigma_{11}\left(\sigma_{22}+\sigma_{33}\right)+a_{2233} \sigma_{22} \sigma_{33}+a_{1112} \sigma_{11}\left(\sigma_{12}+\sigma_{13}\right)+ \\
& a_{1123} \sigma_{11} \sigma_{23}+a_{2212}\left(\sigma_{22}+\sigma_{33}\right)\left(\sigma_{12}+\sigma_{13}\right)+a_{2223} \sigma_{22} \sigma_{23}+a_{3323} \sigma_{33} \sigma_{23}
\end{aligned}
$$

o que diminui o número de incógnitas de 24 para 15. Considerando que para um dado material fossem realizados testes de tração e compressão uniaxial e cisalhamento em todas as direções (considerando o sistema de coordenadas do material), pode-se substituir na equação (87) cada condição de teste e obter as seguintes soluções

$$
\begin{aligned}
& a_{11}=\frac{1}{S_{11}^{t}}-\frac{1}{S_{11}^{c}} \\
& a_{1111}=\frac{1}{S_{11}^{t} S_{11}^{c}} \\
& a_{22}=\frac{1}{S_{22}^{t}}-\frac{1}{S_{22}^{c}} \\
& a_{2222}=\frac{1}{S_{22}^{t} S_{22}^{c}}
\end{aligned}
$$




$$
\begin{aligned}
& a_{12}=a_{23}=0 \\
& a_{1212}=\frac{1}{\left(S_{12}\right)^{2}} \\
& a_{2323}=\frac{1}{\left(S_{23}\right)^{2}}
\end{aligned}
$$

E considerando que o efeito do cisalhamento não pode depender do sinal

$$
a_{1112}=a_{1123}=a_{2212}=a_{2223}=a_{3323}=0
$$

Note que foi considerado que a resistência ao cisalhamento independe do sinal e que as propriedades são de um material transversalmente isotrópico. Pode-se então reescrever a equação (87) como

$$
\begin{aligned}
& f_{T W}=\frac{\sigma_{11}^{2}}{S_{11}^{t} S_{11}^{c}}+\frac{\left(\sigma_{22}^{2}+\sigma_{33}^{2}\right)}{S_{22}^{t} S_{22}^{c}}+\frac{\sigma_{12}^{2}+\sigma_{13}^{2}}{\left(S_{12}\right)^{2}}+\left(\frac{\sigma_{23}}{S_{23}}\right)^{2}+a_{1122} \sigma_{11}\left(\sigma_{22}+\sigma_{33}\right)+ \\
& a_{2233} \sigma_{22} \sigma_{33}+\left(\frac{1}{S_{11}^{t}}-\frac{1}{S_{11}^{c}}\right) \sigma_{11}+\left(\frac{1}{S_{22}^{t}}-\frac{1}{S_{22}^{c}}\right)\left(\sigma_{22}+\sigma_{33}\right)
\end{aligned}
$$

Restando então apenas mais duas constantes a serem calibradas experimentalmente. Segundo Liu e Tsai (1998), na falta de dados experimentais pode-se usar

$$
\begin{aligned}
& a_{1122}=-\frac{1}{\sqrt{S_{11}^{t} S_{11}^{c} S_{22}^{t} S_{22}^{c}}} \\
& a_{2233}=-\frac{1}{S_{22}^{t} S_{22}^{c}}
\end{aligned}
$$

O modelo de Tsai-Hill, que também é bastante popular na literatura, é um caso particular do modelo de Tsai-Wu para quando a lâmina tem resistência à tração igual a resistência à compressão. O mesmo é mais recomendado se a matriz for dúctil. 


\subsubsection{Tensão Plana}
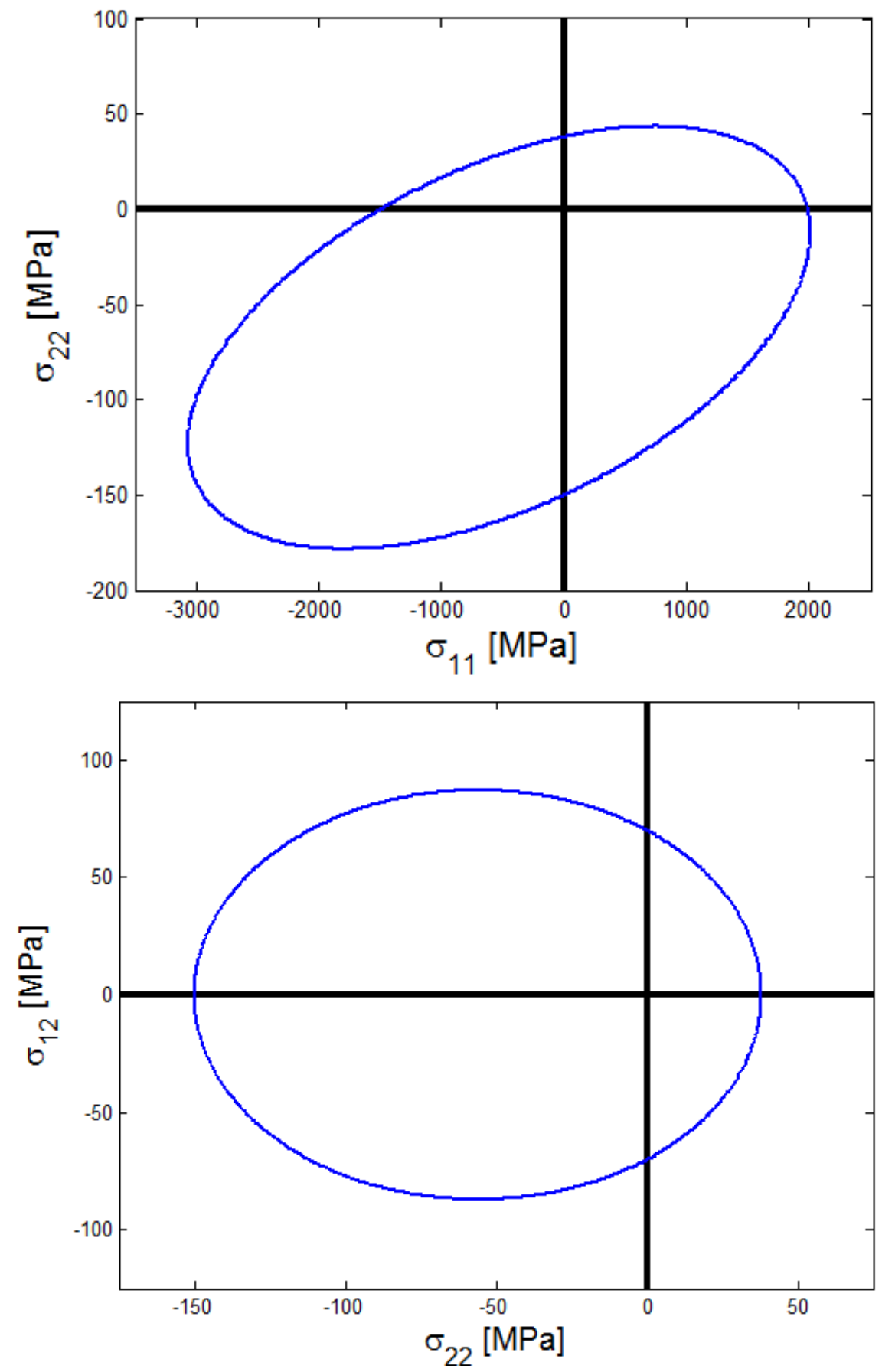

Figura 5 - Principais envelopes de falha pelo modelo de Tsai-Wu

Considerando a hipótese de tensão plana, a Figura 5 mostra exemplos de envelopes de falha $\sigma_{11}-\sigma_{22}$ e $\sigma_{22}-\sigma_{12}$. O envelope de falha $\sigma_{11}-\sigma_{12}$ não será avaliado porque todas 
as informações necessárias já estão expostas nesses dois planos selecionados. O primeiro é o plano das tensões normais e o segundo é o plano utilizado para descrever a falha da matriz nos outros modelos que serão estudados. Lembrando que foram utilizadas as propriedades mostradas no capítulo 1 para obter esses resultados, assim como as mesmas serão aplicadas em todo o desenvolvimento.

Para estudar o efeito do estudo do termo que quantifica o efeito da interação entre as tensões normais no mecanismo de falha, a equação (90) será reescrita como

$$
f_{T W}=\frac{\sigma_{11}^{2}}{S_{11}^{t} S_{11}^{c}}+\frac{\sigma_{22}^{2}}{S_{22}^{t} S_{22}^{c}}+\left(\frac{\sigma_{12}}{S_{12}}\right)^{2}+a_{1122}^{*} \frac{\sigma_{11} \sigma_{22}}{\sqrt{S_{11}^{t} S_{11}^{c} S_{22}^{t} S_{22}^{c}}}+\left(\frac{1}{S_{11}^{t}}-\frac{1}{S_{11}^{c}}\right) \sigma_{11}+\left(\frac{1}{S_{22}^{t}}-\frac{1}{S_{22}^{c}}\right) \sigma_{22}
$$

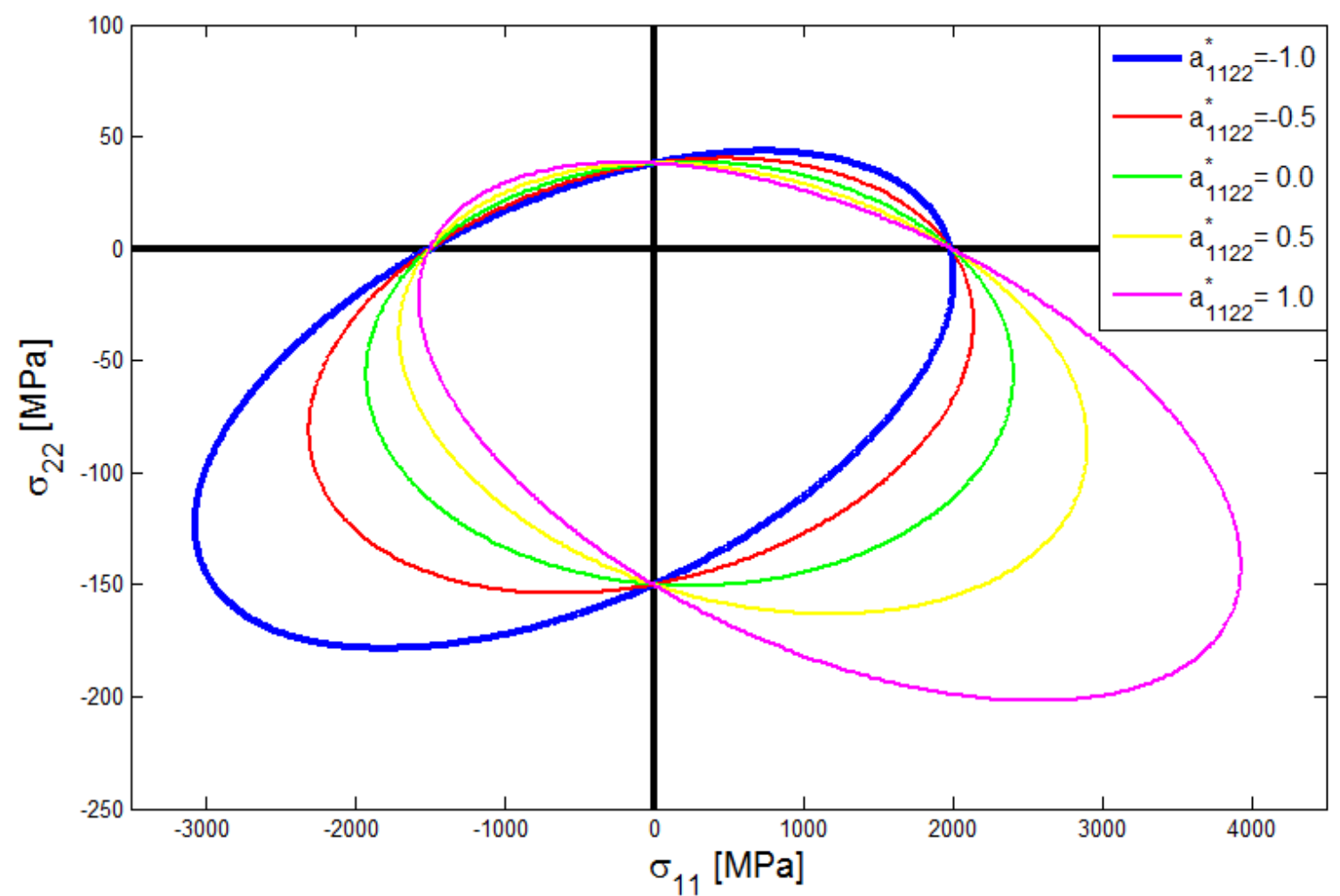

Figura 6 - Influência do parâmetro $a_{1122}^{*}$ no envelope de falha de Tsai-Wu

Note que $a_{1122}=a_{1122}^{*} / \sqrt{S_{11}^{t} S_{11}^{c} S_{22}^{t} S_{22}^{c}}$. Dessa forma, a Figura 6 mostra o efeito de $a_{1122}^{*}$ no envelope de falha. A curva para $a_{1122}^{*}=-1$ está destacada por ser a recomendada. 

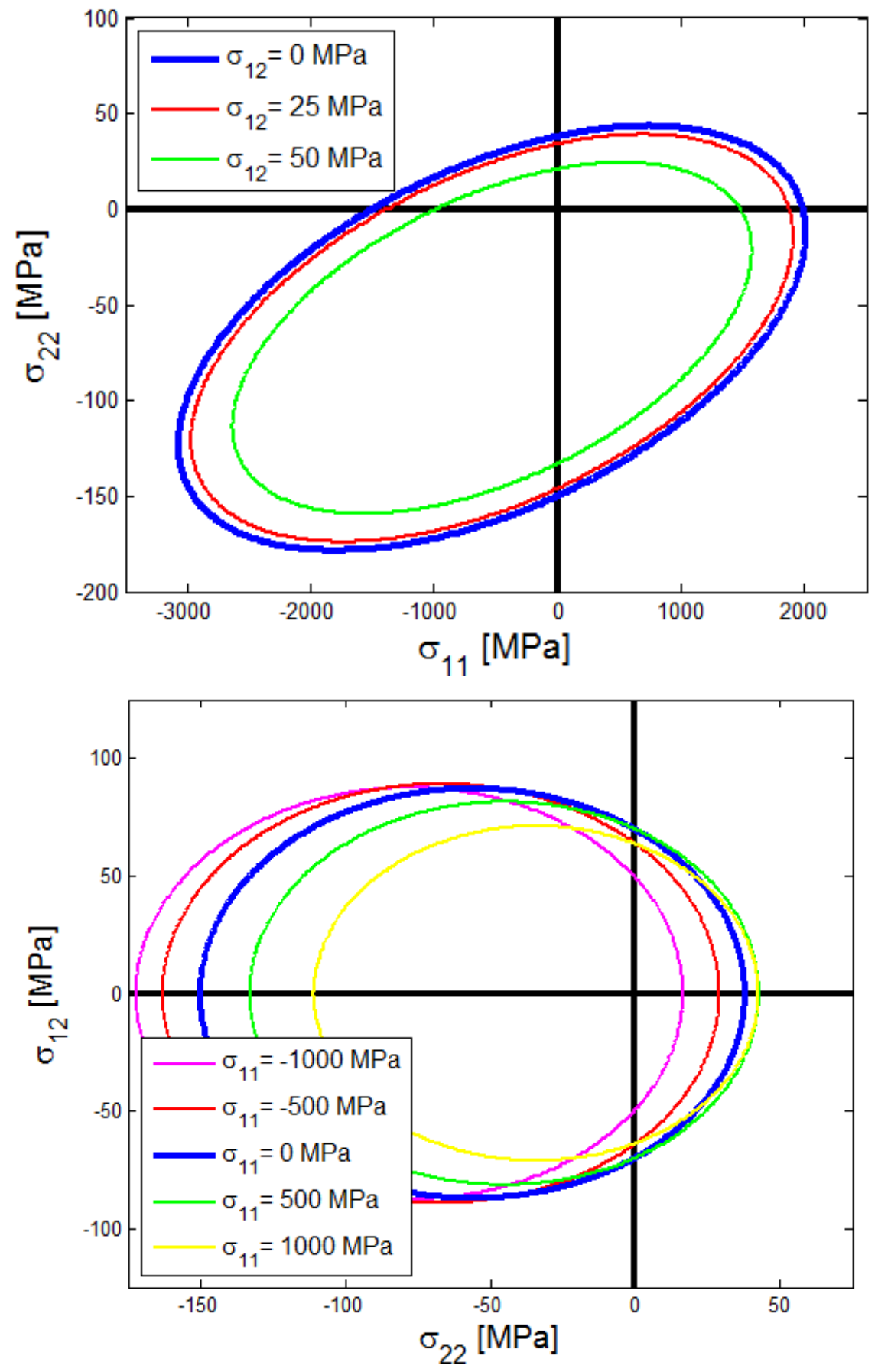

Figura 7 - Efeito da terceira componente de tensão (para tensão plana) nos envelopes de falha de Tsai-Wu.

Pode-se concluir que quanto maior o módulo desse parâmetro, maior é a tendência de não haver falha para valores compressivos de $\sigma_{22}$ e o sinal do mesmo influi em qual quadrante esse envelope de falha irá aumentar. 
Os envelopes de falha das Figuras 5 consideram que a terceira componente da tensão, que não está representada no gráfico, seja nula. Todavia, isso normalmente não é verdade e se torna interessante estudar o efeito dessa componente. Nas Figuras 7, os resultados dessa interação são mostrados. A tensão de cisalhamento diminui o tamanho da superfície da falha no plano $\sigma_{11}-\sigma_{22}$ enquanto a tensão normal na fibra, além de alterar as dimensões da curva, muda também a posição do seu centro no plano $\sigma_{22}-\sigma_{12}$. Fazendo uma analogia à nomenclatura comum em Teoria da Plasticidade, a tensão de cisalhamento atua no plano $\sigma_{11}-\sigma_{22}$ de forma similar ao endurecimento isotrópico e a tensão normal na fibra atua no plano $\sigma_{22}-\sigma_{12}$ semelhantemente a uma condição de endurecimento isotrópico e cinemático combinados.

\subsection{Critério de Puck}

\subsubsection{Caso Geral}

A teoria desenvolvida por Puck e seu grupo tem sido amplamente utilizada, principalmente na Alemanha, incluindo sua implementação em normas (VDI, 2006), e tem se tornado mais popular ao redor do mundo pelo desempenho no WWFE (HINTON et al., 2004) e o livro Analysis of Failure in Fiber Polymer Laminates - The Theory of Alfred Puck (Knops, 2008) que contém a visão detalhada de sua teoria em inglês (a maioria das publicações originais do grupo estão em alemão).

De forma geral, o modelo separa os diferentes mecanismos de falha como sendo falha da fibra e da matriz sob tração ou compressão. Para a falha da fibra, a tradicional Teoria da Tensão Normal Máxima (Jones, 1998) foi utilizada como base. Supondo inicialmente um carregamento trativo (o mesmo desenvolvimento pode ser feito de forma similar para compressão), considera-se que haverá falha se a tensão normal na fibra for igual ao seu limite de resistência, ou seja,

$$
\sigma_{11}^{(f)}=\frac{E_{1}^{(f)}}{E_{1}} S_{11}^{t}
$$

Note que consideram-se as fibras e a matriz como um modelo simplificado de molas em paralelo; os deslocamentos e as deformações devem ser iguais pela compatibilidade 
geométrica. No entanto, como as fibras e a matriz têm propriedades diferentes, alguns efeitos adicionais precisam ser levados em consideração.

A deformação normal na fibra na direção paralela às mesmas pode ser escrita

$$
\varepsilon_{11}^{(f)}=\frac{1}{E_{1}^{(f)}} \sigma_{11}^{(f)}-\frac{\nu_{21}^{(f)}}{E_{2}^{(f)}} m_{f}\left(\sigma_{22}^{(f)}+\sigma_{33}^{(f)}\right)
$$

onde $m_{f}$ é uma constante criada para quantificar o efeito das propriedades elásticas diferentes da matriz e das fibras e pode ser ajustado experimentalmente. $\mathrm{Na}$ falta de dados experimentais, $m_{f}=1.3$ para fibras de vidro e $m_{f}=1.1$ para fibras de carbono podem ser utilizados (ambos os valores consideram matriz polimérica). Lembrando que pela simetria da matriz de rigidez, $\nu_{21}^{(f)} / E_{2}^{(f)}=\nu_{12}^{(f)} / E_{1}^{(f)}$, a tensão normal na fibra pode ser então escrita por

$$
\sigma_{11}^{(f)}=E_{1}^{(f)} \varepsilon_{11}^{(f)}+\nu_{12}^{(f)} m_{f}\left(\sigma_{22}^{(f)}+\sigma_{33}^{(f)}\right)
$$

A deformação normal na direção da fibra pode ser escrita pela relação constitutiva da lâmina como

$$
\varepsilon_{11}=\frac{1}{E_{1}} \sigma_{11}-\frac{\nu_{12}}{E_{1}}\left(\sigma_{22}+\sigma_{33}\right)
$$

Pelas condições de compatibilidade, considerando a equivalência com molas em séria, $\varepsilon_{11}^{(f)}=\varepsilon_{11}$, e por equilíbrio, considerando como molas em paralelo, $\sigma_{22}^{(f)}=\sigma_{22}$ e $\sigma_{33}^{(f)}=\sigma_{33}$, pode-se manipular as equações (93-96) e concluir que, no momento da falha,

$$
\begin{aligned}
& \sigma_{11}^{(f)}=E_{1}^{(f)} \varepsilon_{11}^{(f)}+\nu_{12}^{(f)} m_{f}\left(\sigma_{22}^{(f)}+\sigma_{33}^{(f)}\right)=\frac{E_{1}^{(f)}}{E_{1}} S_{11}^{t} \\
& E_{1}^{(f)}\left(\frac{1}{E_{1}} \sigma_{11}-\frac{\nu_{12}}{E_{1}}\left(\sigma_{22}+\sigma_{33}\right)\right)+\nu_{12}^{(f)} m_{f}\left(\sigma_{22}^{(f)}+\sigma_{33}^{(f)}\right)=\frac{E_{1}^{(f)}}{E_{1}} S_{11}^{t} \\
& \sigma_{11}-\nu_{12}\left(\sigma_{22}+\sigma_{33}\right)+\frac{E_{1}}{E_{1}^{(f)}} \nu_{12}^{(f)} m_{f}\left(\sigma_{22}+\sigma_{33}\right)=S_{11}^{t} \\
& \sigma_{11}+\left(\frac{E_{1}}{E_{1}^{(f)}} \nu_{12}^{(f)} m_{f}-\nu_{12}\right)\left(\sigma_{22}+\sigma_{33}\right)=S_{11}^{t}
\end{aligned}
$$

As funções que descrevem a falha da fibra do modelo de Puck podem ser então escritas como 


$$
\begin{aligned}
& f_{P}^{(f, t)}=\frac{1}{S_{11}^{t}}\left|\sigma_{11}+\left(\frac{E_{1}}{E_{1}^{(f)}} \nu_{12}^{(f)} m_{f}-\nu_{12}\right)\left(\sigma_{22}+\sigma_{33}\right)\right| \\
& f_{P}^{(f, c)}=\frac{1}{S_{11}^{c}}\left|\sigma_{11}+\left(\frac{E_{1}}{E_{1}^{(f)}} \nu_{12}^{(f)} m_{f}-\nu_{12}\right)\left(\sigma_{22}+\sigma_{33}\right)\right|
\end{aligned}
$$

Baseado na experiência do grupo, Puck e Schurmann (1998) propuseram que para carregamento compressivo a seguinte modificação empírica seja adotada

$$
f_{P}^{(f, c)}=\frac{1}{S_{11}^{c}}\left|\sigma_{11}+\left(\frac{E_{1}}{E_{1}^{(f)}} \nu_{12}^{(f)} m_{f}-\nu_{12}\right)\left(\sigma_{22}+\sigma_{33}\right)\right|+\left(10 \frac{\sigma_{12}}{G_{12}}\right)^{2}
$$

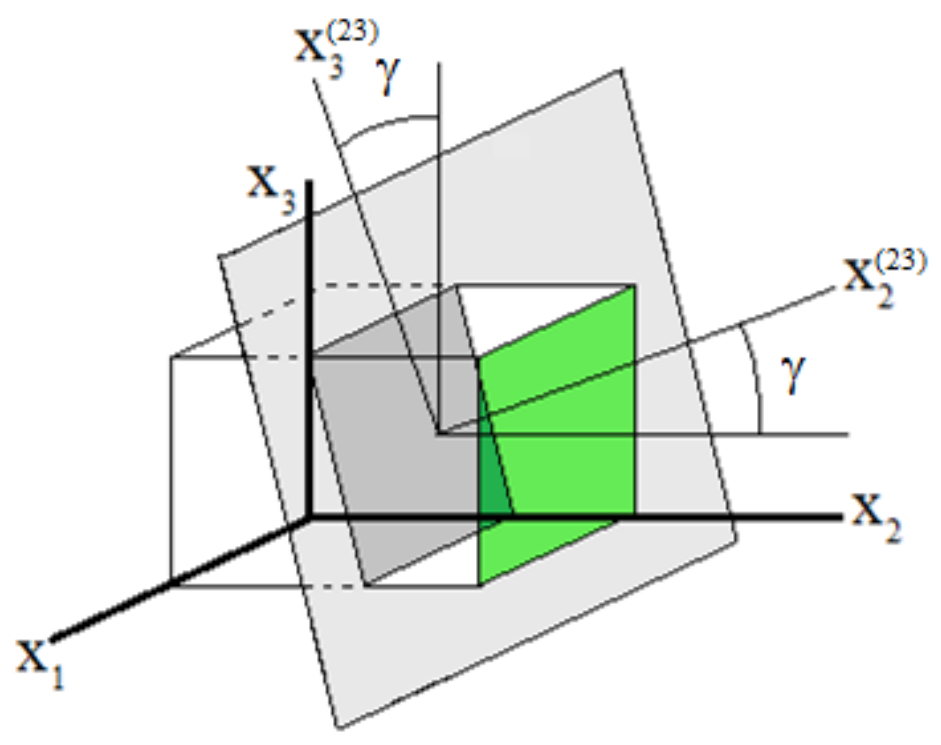

Figura 8 - Plano crítico no modelo de falha da matriz

Experimentalmente, pode-se verificar as falha da matriz com características frágeis, sem plasticidade macroscópica considerável. Com esse conhecimento prévio, o modelo de falha da matriz usa como base o critério de Mohr. Uma das hipóteses fundamentais do presente modelo é que a falha ocorre exclusivamente pelo estado de tensões atuantes no plano crítico, o que consequentemente resulta em uma previsão adicional: o plano da fratura, e não apenas se a mesma ocorrerá ou não. 
O plano crítico tem uma componente coincidente com a direção $x_{1}$ e a outra precisa ser encontrada em qualquer direção no plano $x_{2}-x_{3}$, para isso o índice superior ${ }^{(23)}$ será utilizado. As componentes de tensão nesse plano podem ser obtidas por

$$
\sigma_{i j}^{(23)}=\lambda_{i k} \lambda_{j l} \sigma_{k l}
$$

onde $i, j, k, l=1,2,3$ e o ângulo entre $x_{2}$ e $x_{2}^{(23)}$ é $\gamma$, como mostrado na Figura 8.

Considerando então o plano $x_{1}-x_{2}^{(23)}$, as tensões $\sigma_{11}, \sigma_{12}^{(23)}, \sigma_{22}^{(23)}$ e $\sigma_{23}^{(23)}$ podem estar atuando no caso geral. Tratando as tensões de cisalhamento como vetores, a tensão de cisalhamento efetiva nesse plano é (Knops, 2008)

$$
\sigma_{12}^{\Phi}=\left[\left(\sigma_{12}^{(23)}\right)^{2}+\left(\sigma_{23}^{(23)}\right)^{2}\right]^{1 / 2}
$$

O índice $\Phi$ foi adotado por causa de seguinte definição

$$
\Phi=a \tan \left(\frac{\sigma_{12}^{(23)}}{\sigma_{23}^{(23)}}\right)
$$

Usando as equações (100-102) como base, as seguintes funções de falha podem ser escritas

$$
\begin{aligned}
& f_{P}^{(m, c)}=\left(\frac{\sigma_{12}^{\Phi}}{S_{12}^{\Phi}}\right)^{2}+2 \frac{p_{\Phi}^{c}}{S_{12}^{\Phi}} \sigma_{22}^{(23)}+\left(\frac{\sigma_{11}}{X_{11}}\right)^{n_{i t}} \\
& f_{P}^{(m, t)}=\left(\frac{\sigma_{12}^{\Phi}}{S_{12}^{\Phi}}\right)^{2}+2 \frac{p_{\Phi}^{t}}{S_{12}^{\Phi}} \sigma_{22}^{(23)}+\left(1-2 p_{\Phi}^{t} \frac{S_{22}^{t}}{S_{12}^{\Phi}}\right)\left(\frac{\sigma_{22}^{(23)}}{S_{22}^{t}}\right)^{2}+\left(\frac{\sigma_{11}}{X_{11}}\right)^{n_{i t}}
\end{aligned}
$$

onde $X_{11}=1.1 S_{11}^{t}$ se $\sigma_{11} \geq 0$ e $X_{11}=-1.1 S_{11}^{c}$ se $\sigma_{11}<0$ e $n_{i t}$ é um parâmetro que pode ser calibrado experimentalmente. De acordo com Puck e Schürmann (1998), tipicamente $6<n_{i t}<8$, para esse estudo será considerado $n_{i t}=8 . S_{12}^{\Phi}, \quad p_{\Phi}^{c}$ e $p_{\Phi}^{t}$ são definidos de forma simplificada - uma definição mais genérica pode ser encontrada em Deuschle e Puck (2013) - como

$$
\begin{aligned}
& \left(\frac{\sigma_{12}^{\Phi}}{S_{12}^{\Phi}}\right)^{2}=\left(\frac{\sigma_{12}^{(23)}}{S_{12}}\right)^{2}+\left(\frac{\sigma_{23}^{(23)}}{S_{23}^{(23)}}\right)^{2} \\
& p_{\Phi}^{c}=S_{12}^{\Phi}\left(\frac{p_{12}^{c}}{S_{12}}\right)
\end{aligned}
$$




$$
p_{\Phi}^{t}=S_{12}^{\Phi}\left(\frac{p_{12}^{t}}{S_{12}}\right)
$$

Onde, na ausência de dados experimentais, $p_{12}^{c}$ e $p_{12}^{t}$ podem ser assumidos iguais a 0.25 e 0.3 para fibras de vidro ou 0.3 e 0.35 para fibras de carbono (Knops, 2008), e

$$
S_{23}^{(23)}=\frac{S_{12}}{2 p_{12}^{c}}\left[\left(1+2 p_{12}^{c} \frac{S_{22}^{c}}{S_{12}}\right)^{1 / 2}-1\right]
$$

Apesar do efeito da tensão normal na direção da fibra não ser inicialmente considerado na modelagem da falha da matriz, foi verificado que altos níveis de deformação acumulam danos consideráveis na matriz antes da falha da fibra, sendo então acrescentados os últimos termos nas equações (103.a,b). Usando as equações anteriores, o modelo de Puck está inteiramente estabelecido, entretanto, um estudo detalhado sobre o caso de tensão plana será realizado a seguir devido à sua importância prática e para obter melhor entendimento do significado físico de alguns parâmetros ajustáveis.

\subsubsection{Tensão Plana}

As equações que descrevem a falha da fibra podem ser escritas de forma semelhante mostrada na seção anterior como

$$
\begin{aligned}
& f_{P}^{(f, t)}=\frac{1}{S_{11}^{t}}\left|\sigma_{11}+\left(\frac{E_{1}}{E_{1}^{(f)}} \nu_{12}^{(f)} m_{f}-\nu_{12}\right) \sigma_{22}\right| \\
& f_{P}^{(f, c)}=\frac{1}{S_{11}^{c}}\left|\sigma_{11}+\left(\frac{E_{1}}{E_{1}^{(f)}} \nu_{12}^{(f)} m_{f}-\nu_{12}\right) \sigma_{22}\right|+\left(10 \frac{\sigma_{12}}{G_{12}}\right)^{2}
\end{aligned}
$$

Se o material estiver submetido a um estado plano de tensões em relação ao sistema global, as tensões no plano crítico da falha da matriz podem ser escritas como

$$
\begin{aligned}
& \sigma_{22}^{(23)}=\sigma_{22} \cos ^{2} \gamma \\
& \sigma_{12}^{(23)}=\sigma_{12} \cos \gamma \\
& \sigma_{23}^{(23)}=-\sigma_{22} \sin \gamma \cos \gamma
\end{aligned}
$$

Considera-se, então, que três diferentes tipos de falha podem acontecer, denominados modos A, B, e C. No modo A, a matriz está sob tração e o plano crítico 
ocorre com $\gamma=0^{\circ}$, como normalmente pode ser assumido para materiais frágeis. Nos modos B e C, a matriz está sob compressão e o que diferencia os dois é o plano crítico; no modo $\mathrm{B} \gamma=0^{\circ}$ e no modo $\mathrm{C} 0^{\circ} \leq \gamma \leq 180^{\circ}$ precisa ser encontrado.

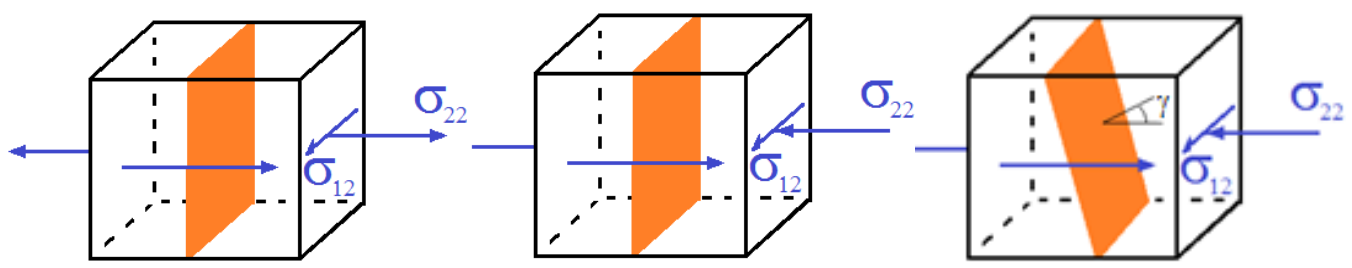

Figura 9 - Modos de falha da matriz para tensão plana

Para o modo A, as seguintes relações podem ser obtidas

$$
\begin{aligned}
& \sigma_{22}^{(23)}=\sigma_{22} \\
& \sigma_{12}^{(23)}=\sigma_{12} \\
& \sigma_{23}^{(23)}=0 \\
& \sigma_{12}^{\Phi}=\sigma_{12} \\
& \left(\frac{\sigma_{12}^{\Phi}}{S_{12}^{\Phi}}\right)^{2}=\frac{\sigma_{12}}{S_{12}}
\end{aligned}
$$

E então pode-se escrever o critério de falha como

$$
f_{P}^{(m, A)}=\left(\frac{\sigma_{12}}{S_{12}}\right)^{2}+\left(\frac{\sigma_{22}}{S_{22}^{t}}\right)^{2}+2 \frac{p_{12}^{t}}{S_{12}} \sigma_{22}\left(1-\frac{\sigma_{22}}{S_{22}^{t}}\right)+\left(\frac{\sigma_{11}}{X_{11}}\right)^{n_{i t}}
$$

Para o modo B, as equações (108.a-e) continuam sendo válidas, sendo necessário apenas aplicar a equação do critério de falha para compressão

$$
f_{P}^{(m, B)}=\left(\frac{\sigma_{12}}{S_{12}}\right)^{2}+2 \frac{p_{12}^{c}}{S_{12}} \sigma_{22}+\left(\frac{\sigma_{11}}{X_{11}}\right)^{n_{i t}}
$$

Para o modo C,

$$
\begin{aligned}
& \sigma_{22}^{(23)}=\sigma_{22} \cos ^{2} \gamma \\
& \sigma_{12}^{(23)}=\sigma_{12} \cos \gamma \\
& \sigma_{23}^{(23)}=-\sigma_{22} \sin \gamma \cos \gamma
\end{aligned}
$$




$$
\begin{aligned}
& \sigma_{12}^{\Phi}=\cos \gamma\left(\sigma_{12}{ }^{2}+\sigma_{22}{ }^{2} \sin ^{2} \gamma\right)^{1 / 2} \\
& \left(\frac{\sigma_{12}^{\Phi}}{S_{12}^{\Phi}}\right)^{2}=\left(\frac{\sigma_{12} \cos \gamma}{S_{12}}\right)^{2}+\left(\frac{\sigma_{22} \sin \gamma \cos \gamma}{S_{23}^{(23)}}\right)^{2}
\end{aligned}
$$
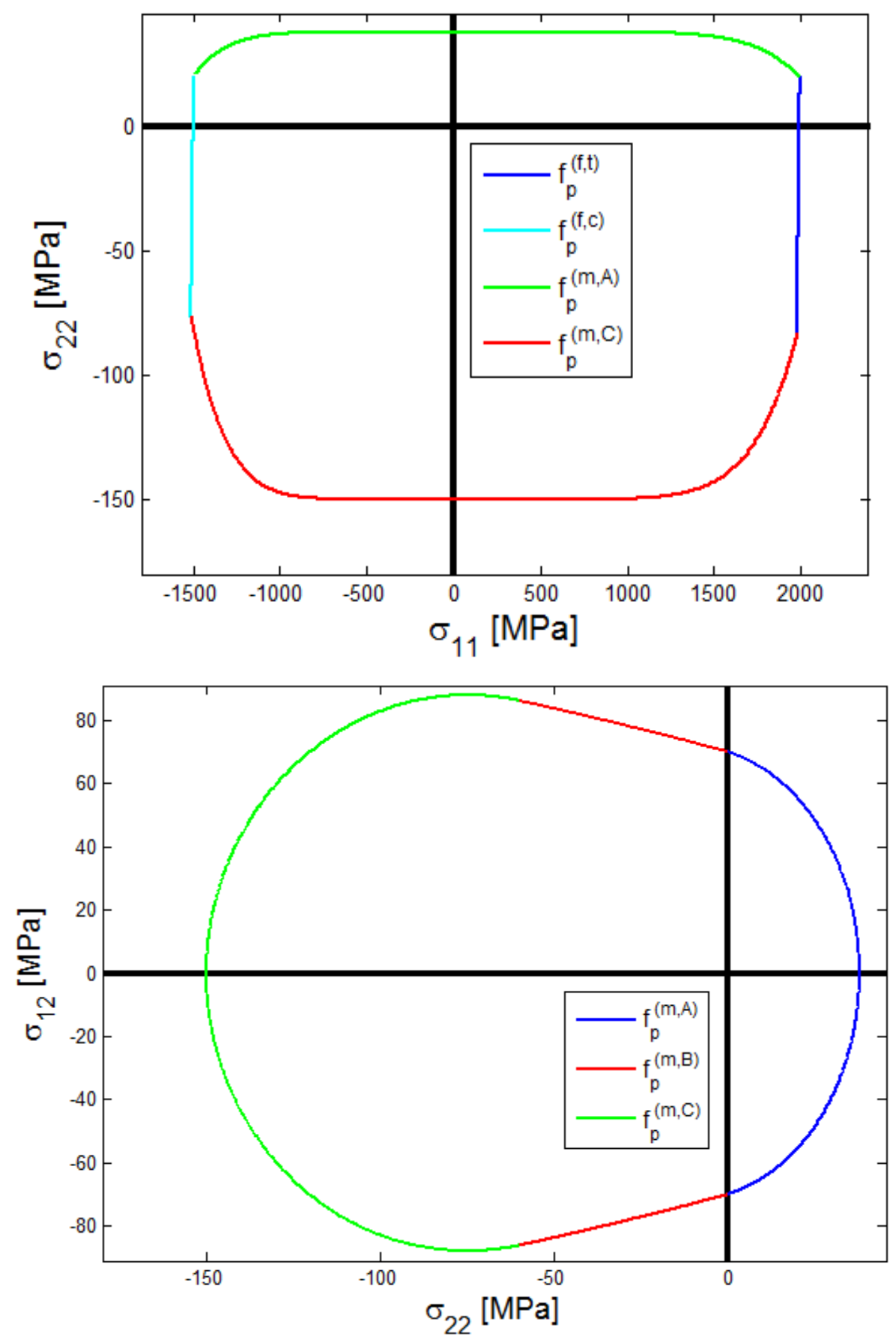

Figura 10 - Principais envelopes de falha pelo modelo de Puck 
E a função que descreve a falha pode ser, então, escrita como

$$
f_{P}^{(m, C)}=\left(\frac{\sigma_{12} \cos \gamma}{S_{12}}\right)^{2}+\left(\frac{\sigma_{22} \sin \gamma \cos \gamma}{S_{23}^{(23)}}\right)^{2}+2 \frac{p_{12}^{c}}{S_{12}} \sigma_{22} \cos ^{2} \gamma+\left(\frac{\sigma_{11}}{X_{11}}\right)^{n_{i t}}
$$

Como no plano crítico é um ponto máximo da função

$$
\frac{d}{d \gamma} f_{P}^{(m, C)}=0
$$

Como $\sin \gamma=0$ é a solução do modo B e assumindo que exista a solução não trivial $(\cos \gamma \neq 0)$, de acordo com Knops (2008)

$$
\gamma_{C}=a \cos \left\{\left[\frac{1}{2\left(1+p_{12}^{c}\right)}\left(\left(\frac{S_{23}^{(23)}}{S_{12}} \frac{\sigma_{12}}{\sigma_{22}}\right)^{2}+1\right)\right]^{\frac{1}{2}}\right\}
$$

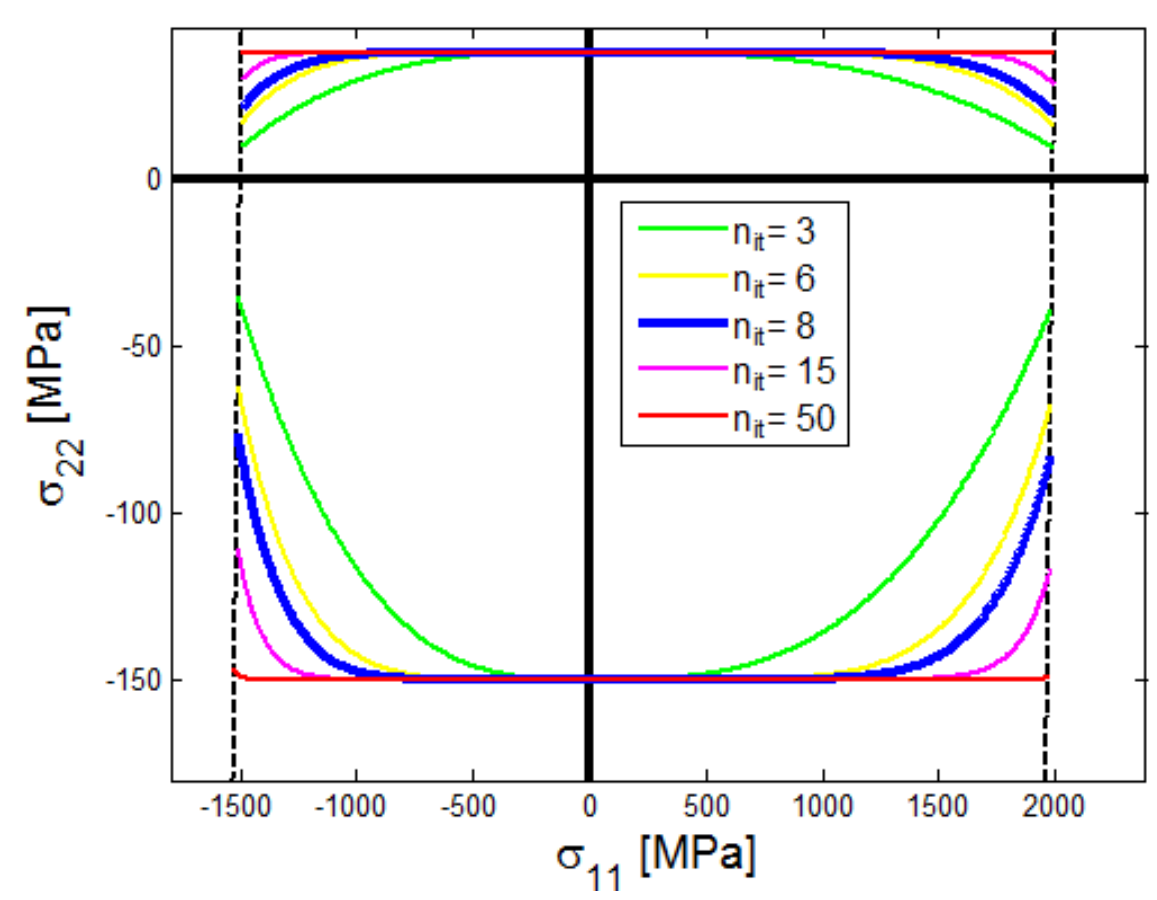

Figura 11 - Influência do parâmetro $n_{i t}$ no envelope de falha de Puck

Dessa forma, todas as equações necessárias para calcular o plano crítico para o caso de tensão plana estão explicitadas e a busca numérica pode ser eliminada, diminuindo consideravelmente o custo computacional. 
No momento de transição entre a falha em modo $\mathrm{B}$ e modo C (PUCK; SCHÜRMANN, 1998)

$$
\begin{aligned}
& \sigma_{22}=-S_{23}^{(23)} \\
& \sigma_{12}=S_{12}\left(1+2 p_{12}^{c} \frac{S_{23}^{(23)}}{S_{12}}\right)^{1 / 2}
\end{aligned}
$$
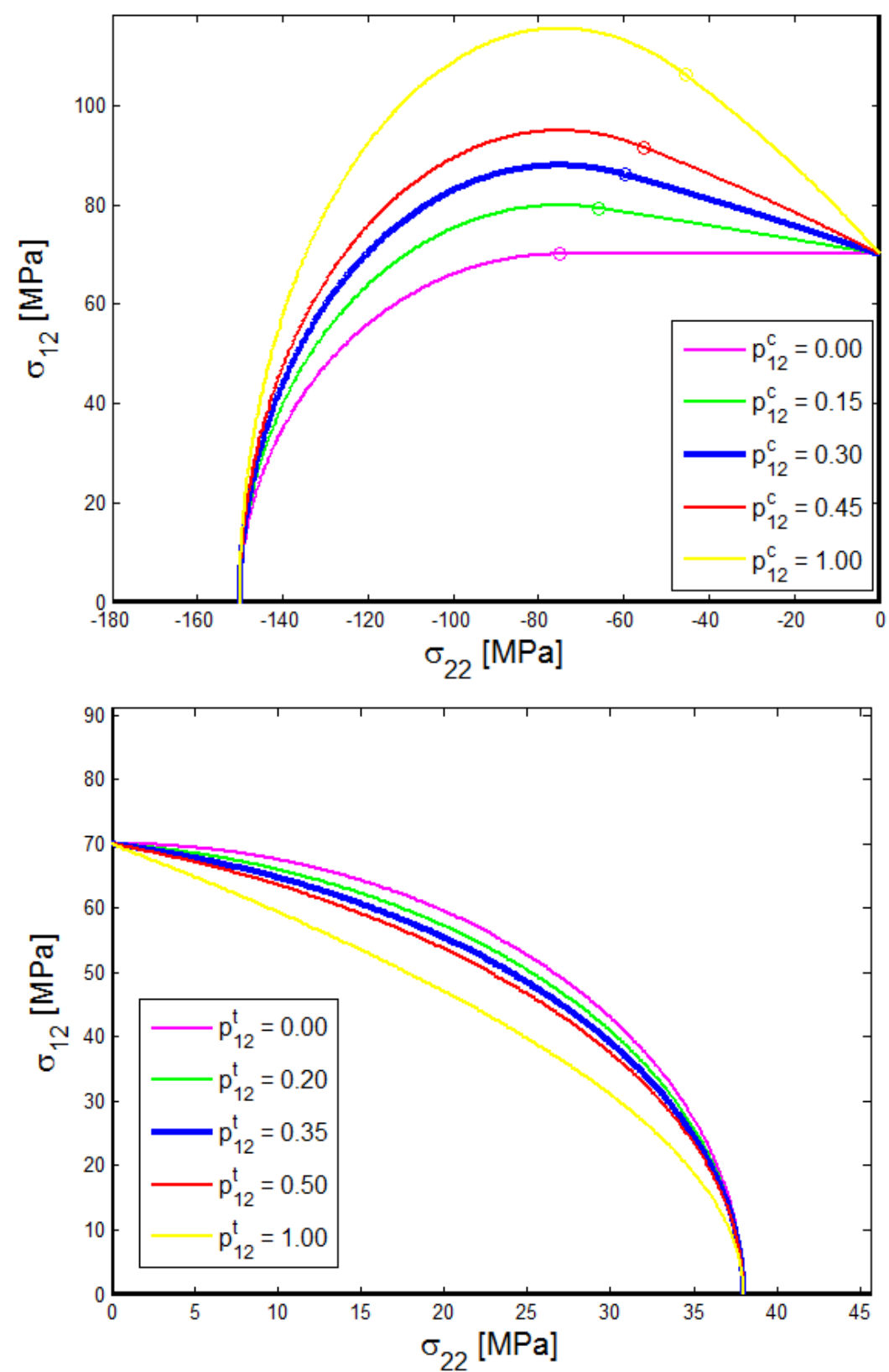

Figura 12 - Influência dos parâmetros $p_{12}^{c}$ e $p_{12}^{t}$ no envelope de falha de Puck 
Uma vez que as equações são obtidas, pode-se facilmente gerar envelopes de falha nos planos $\sigma_{11}-\sigma_{22}$ e $\sigma_{22}-\sigma_{12}$ como mostrados nas Figuras 10.
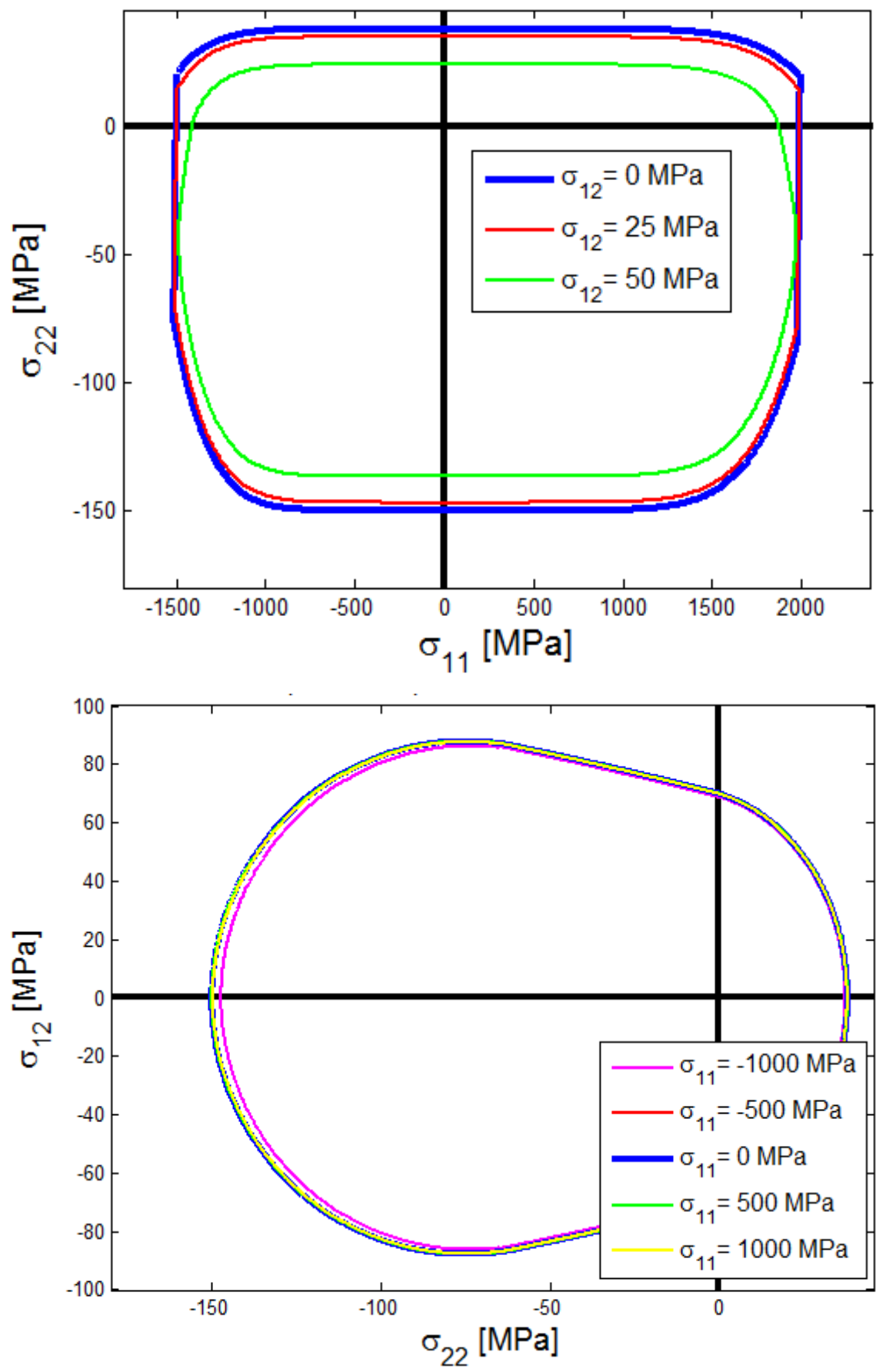

Figura 13 - Efeito da terceira componente de tensão (para tensão plana) nos envelopes de falha de Puck 
Três parâmetros são ajustáveis experimentalmente no modelo de Puck: $n, p_{12}^{c}$ e $p_{12}^{t}$. Os gráficos das Figuras 10 foram obtidos utilizando 8, 0.30 e 0.35, respectivamente. A seguir, será mostrada uma análise de sensibilidade de cada uma dessas grandezas.

O expoente $n$ quantifica a influência de $\sigma_{11}$ na falha da matriz; quanto maior esse valor, menos sensível é o material ao acúmulo de dano, tendendo então a falhar sem dano progressivo aparente.

$p_{12}^{c}$ e $p_{12}^{t}$ são definidos fisicamente como (Knops, 2008)

$$
\begin{aligned}
& p_{12}^{c}=-\left.\left(\frac{\partial \sigma_{12}}{\partial \sigma_{22}}\right)\right|_{\sigma_{22} \rightarrow 0^{-}} \\
& p_{12}^{t}=-\left.\left(\frac{\partial \sigma_{12}}{\partial \sigma_{22}}\right)\right|_{\sigma_{22} \rightarrow 0^{+}}
\end{aligned}
$$

Tornando, então, claro o seu significado físico, como ilustrado na Figura 12.

Na Figura 13, os resultados da interação entre tensões no envelope de falha são mostrados. Uma importante conclusão é que o modelo de Puck se mostra muito menos sensível à essa terceira componente de tensão do que o de Tsai-Wu.

\subsection{Critério LaRC05}

\subsubsection{Caso geral}

Enquanto os modelos anteriores foram inicialmente desenvolvidos na metade do século passado, o critério LaRC05 tem sido desenvolvido desde o início dos anos 2000, todavia, tem demostrado boa capacidade de descrever mecanismos de dano. Embora Pinho et al. (2012) mostrarem dados experimentais que indicam não-linearidades em relação a $E_{2}$ e $G_{12}$ para elevados níveis de deformação e os mesmos recomendem levar esses efeitos em consideração, no presente trabalho será assumida a resposta linear para tornar aplicável o desenvolvimento do formalismo de Stroh mostrado anteriormente, o que é uma hipótese razoável uma vez que o estudo será baseado na filosofia damage-free (FPF).

As principais referências que serviram de base para o modelo que será apresentado foram Pinho et al. (2005) e Pinho et al. (2012). 
Para a falha da matriz o modelo de Puck é usado como base. Baseado também na ideia de que a fratura ocorre no plano crítico e que o carregamento trativo gera mais dano do que o compressivo em materiais frágeis (como é o caso da matriz), a função que descreve a falha da matriz pode ser escrita como

$$
f_{L}^{(m)}=\left(\frac{\sigma_{12}^{(23)}}{S_{12}-b_{L} \sigma_{22}^{(23)}}\right)^{2}+\left(\frac{\sigma_{23}^{(23)}}{S_{23}^{(23)}-b_{T} \sigma_{22}^{(23)}}\right)^{2}+\left(\frac{\max \left\{0, \sigma_{22}^{(23)}\right\}}{S_{22}^{t}}\right)^{2}
$$

onde $b_{L}$ e $b_{T}$ são experimentalmente calibrados e $S_{23}^{(23)}$ é a resistência ao cisalhamento no plano $x_{1}-x_{2}^{(23)}$.

Note que a única diferenciação entre o carregamento trativo e o compressivo é o último termo. Esse termo considera implicitamente que os defeitos e cavidades intrínsecos na microestrutura só se propagam sob tração, assim como a quebra das cadeias poliméricas - para relembrar a ideia do plano crítico ver a Figura 8 e a equação (100).

Uma das grandes vantagens do LaRC05 é levar em consideração todas as possíveis conclusões resultantes de observações em mesoescala mas manter a análise de tensões em escala macroscópica.

De acordo com Dávila et al. (2005)

$$
\begin{aligned}
& b_{T}=-\frac{1}{\tan 2 \theta_{0}} \\
& S_{23}^{(23)}=S_{22}^{c} \cos \theta_{0}\left(\sin \theta_{0}+\frac{\cos \theta_{0}}{\tan 2 \theta_{0}}\right)
\end{aligned}
$$

onde pode-se assumir $51^{\circ}<\theta_{0}<55^{\circ}$ na falta de dados experimentais. Será assumido $\theta_{0}=53^{\circ}$ e $b_{L}=0.082$, e então $b_{T}$ e $S_{23}^{(23)}$ podem ser obtidos.

Para a fibra sob tração, o Critério da Máxima Tensão Normal pode ser diretamente aplicado, ou seja,

$$
f_{L}^{(f, t)}=\frac{\sigma_{11}}{S_{11}^{t}}
$$

Para a fibra sob compressão, dois diferentes mecanismos podem ocorrer: microflambagem e separação das fibras; entretanto, para a análise linear (sem progressão do dano) a modelagem de ambos os fenômenos é a mesma. 
Se todas as fibras estiverem inicialmente alinhadas, uma rotação de $\phi$ no plano $x_{2}-x_{3}$ deverá ser considerada, com $0^{\circ} \leq \phi \leq 180^{\circ}$, para procurar o plano crítico que maximizará a função. Uma vez que esse procedimento seja executado, o plano $x_{1}-x_{2}^{(\phi)}$ deverá ser analisado. Para calcular as componentes de tensões atuantes no plano $x_{2}^{(\phi)}-x_{3}^{(\phi)}$, pode-se utilizar a seguinte transformação

$$
\sigma_{i j}^{(\phi)}=\lambda_{i k} \lambda_{j l} \sigma_{k l}
$$

onde $\phi$ é o ângulo entre $x_{2}^{(\phi)}$ e $x_{2}$. Entretanto, o desalinhamento inicial das fibras é um defeito inevitável resultante dos processos de fabricação e deve ser considerado. Então quando o mecanismo de falha sob compressão é analisado, este desalinhamento contribui significativamente para a instabilidade da fibra por causa de sua inicial deflexão, necessitando, assim, de outra transformação no plano $x_{1}-x_{2}^{(\phi)}$ para considerar as componentes das tensões na direção do desalinhamento, ou seja,

$$
\sigma_{i j}^{(m i s)}=\lambda_{i k} \lambda_{j l} \sigma_{k l}^{(\phi)}
$$

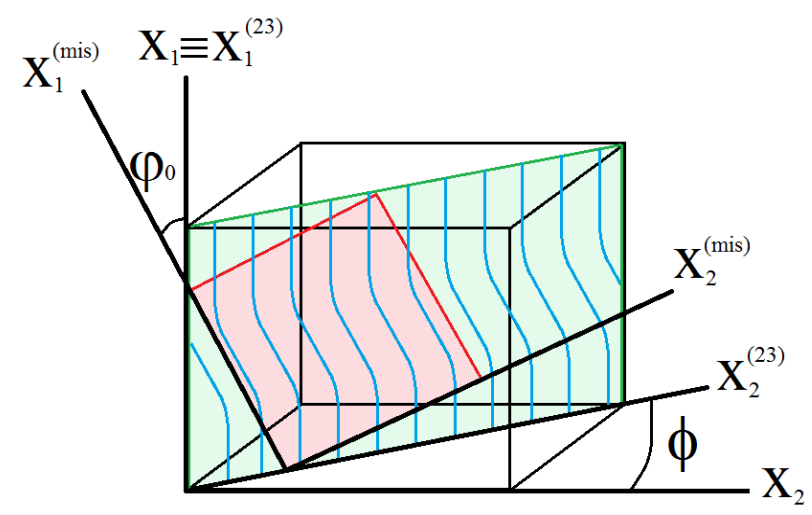

Figura 14 - Plano crítico considerando o desalinhamento das fibras

Mas a transformação anterior não é conhecida porque o ângulo de desalinhamento ( $\varphi$ ) ainda é uma incógnita. De acordo com Pinho et al. (2005) e Pinho et al. (2006), o seguinte procedimento pode ser seguido para obter o desalinhamento.

Considerando o desalinhamento inicial e a rotação devido ao cisalhamento, pode-se escrever

$$
\varphi=\operatorname{sign}\left(\sigma_{12}^{(\phi)}\right) \varphi_{0}+2 \varepsilon_{12}^{(m i s)}
$$


e a função de falha pode ser escrita como

$$
f_{L}^{(f, c)}=\left(\frac{\sigma_{12}^{(m i s)}}{S_{12}-b_{L} \sigma_{22}^{(m i s)}}\right)^{2}+\left(\frac{\sigma_{23}^{(m i s)}}{S_{23}^{(23)}-b_{T} \sigma_{22}^{(m i s)}}\right)^{2}+\left(\frac{\max \left\{0, \sigma_{22}^{(m i s)}\right\}}{S_{22}^{t}}\right)^{2}
$$

A ilustração do plano de falha está mostrada na Figura 14.

Considerando que o material falha sob compressão pura quando $\sigma_{11}=S_{11}^{c}$, o ângulo mostrado na equação (122) é $\varphi=\varphi^{c}$ e as componentes das tensões mostradas na equação (123) são, independentemente do valor de $\phi$,

$$
\begin{aligned}
& \sigma_{22}^{(m i s)}=\lambda_{21} \lambda_{21} \sigma_{11}=-S_{11}^{c} \sin ^{2} \varphi^{c} \\
& \sigma_{12}^{(m i s)}=\lambda_{11} \lambda_{21} \sigma_{11}=S_{11}^{c} \sin \varphi^{c} \cos \varphi^{c} \\
& \sigma_{23}^{(m i s)}=0
\end{aligned}
$$

Substituindo na equação (123), tem-se

$$
\frac{S_{11}^{c} \sin \varphi^{c} \cos \varphi^{c}}{S_{12}+b_{L} S_{11}^{c} \sin ^{2} \varphi^{c}}=1
$$

E reorganizando

$$
b_{L} \sin ^{2} \varphi^{c}-\sin \varphi^{c} \cos \varphi^{c}+\frac{S_{12}}{S_{11}^{c}}=0
$$

Dividindo todas as parcelas por $\cos ^{2} \varphi_{0}$ e usando a identidade $\frac{1}{\cos ^{2} \varphi^{c}}=\tan ^{2} \varphi^{c}+1$

$\left(\frac{S_{12}}{S_{11}^{c}}+b_{L}\right) \tan ^{2} \varphi^{c}-\tan \varphi^{c}+\frac{S_{12}}{S_{11}^{c}}=0$

que tem como solução

$$
\varphi^{c}=a \tan \left[\frac{1-\sqrt{1-4\left(\frac{S_{12}}{S_{11}^{c}}+b_{L}\right) \frac{S_{12}}{S_{11}^{c}}}}{2\left(\frac{S_{12}}{S_{11}^{c}}+b_{L}\right)}\right]
$$

Usando que para pequenos ângulos $\varphi^{c} \cong \tan \varphi^{c}$ 


$$
\varphi^{c}=\left[\frac{1-\sqrt{1-4\left(\frac{S_{12}}{S_{11}^{c}}+b_{L}\right) \frac{S_{12}}{S_{11}^{c}}}}{2\left(\frac{S_{12}}{S_{11}^{c}}+b_{L}\right)}\right]
$$

Para os valores mostrados na Tabela $1, \varphi^{c}=2.688^{\circ}$, utilizando a equação (128) e $\varphi^{c}=2.69^{\circ}$ utilizando a equação (129), o que ilustra que o erro em considerar as aproximações para pequenos ângulos é pequeno, todavia como a solução exata é obtida e o custo do seu cálculo não justifica a utilização da equação (129), a equação (128) deve ser utilizada.

Na equação (122) o valor de $\varphi_{0}$ ainda precisa ser determinado. No momento da falha por compressão,

$$
\varphi^{c}=\varphi_{0}+\frac{S_{11}^{c} \sin \varphi^{c} \cos \varphi^{c}}{G_{12}}
$$

Como discutido anteriormente, pode-se utilizar a aproximação de pequenos ângulos para $\varphi^{c}$ e então a equação (130) pode ser reescrita como

$$
\varphi_{0} \cong\left(1-\frac{S_{11}^{c}}{G_{12}}\right) \varphi^{c}=\left(1-\frac{S_{11}^{c}}{G_{12}}\right) a \tan \left[\frac{1-\sqrt{1-4\left(\frac{S_{12}}{S_{11}^{c}}+b_{L}\right) \frac{S_{12}}{S_{11}^{c}}}}{2\left(\frac{S_{12}}{S_{11}^{c}}+b_{L}\right)}\right]
$$

E de forma genérica, a parcela da rotação na equação (122) pode ser reescrita como

$$
2 \varepsilon_{12}^{(m i s)}=c_{12 i j}^{(m i s)} \sigma_{i j}^{(m i s)}=2 c_{1212}^{(m i s)} \sigma_{12}^{(m i s)}
$$

Note que na direção do desalinhamento a coordenada $x_{1}^{(m i s)}$ é paralela às fibras, logo todas as outras componentes do tensor de flexibilidade se tornam nulas no somatório tensorial.

A tensão de cisalhamento pode ser calculada como

$$
\sigma_{12}^{(m i s)}=\left(\lambda_{11} \lambda_{21} \sigma_{11}^{(\phi)}+\lambda_{12} \lambda_{22} \sigma_{22}^{(\phi)}+\left(\lambda_{12} \lambda_{21}+\lambda_{11} \lambda_{22}\right) \sigma_{12}^{(\phi)}\right)
$$

onde

$$
\sigma_{i j}^{(\phi)}=\left(\begin{array}{l}
\lambda_{i 1} \lambda_{j 1} \sigma_{11}+\lambda_{i 2} \lambda_{j 2} \sigma_{22}+\lambda_{i 3} \lambda_{j 3} \sigma_{33}+ \\
\left(\lambda_{i 2} \lambda_{j 1}+\lambda_{i 1} \lambda_{j 2}\right) \sigma_{12}+\left(\lambda_{i 3} \lambda_{j 1}+\lambda_{i 1} \lambda_{j 3}\right) \sigma_{13}+\left(\lambda_{i 3} \lambda_{j 2}+\lambda_{i 2} \lambda_{j 3}\right) \sigma_{23}
\end{array}\right)
$$


ou, explicitando,

$$
\begin{aligned}
& \sigma_{11}^{(\phi)}=\sigma_{11} \\
& \sigma_{22}^{(\phi)}=\sigma_{22} \cos ^{2} \phi+\sigma_{33} \sin ^{2} \phi+2 \sigma_{23} \sin \phi \cos \phi \\
& \sigma_{12}^{(\phi)}=\sigma_{12} \cos \phi+\sigma_{13} \sin \phi \\
& \sigma_{12}^{(m i s)}=\left(\sigma_{22}^{(\phi)}-\sigma_{11}^{(\phi)}\right) \sin \varphi \cos \varphi+\left(\cos ^{2} \varphi-\sin ^{2} \varphi\right) \sigma_{12}^{(\phi)}
\end{aligned}
$$

e considerando pequenos ângulos

$$
\sigma_{12}^{(m i s)}=\left(\sigma_{22} \cos ^{2} \phi+\sigma_{33} \sin ^{2} \phi+\sigma_{23} \sin 2 \phi-\sigma_{11}\right) \varphi+\left(\sigma_{12} \cos \phi+\sigma_{13} \sin \phi\right)
$$

a equação (122) pode, então, ser escrita como

$$
\begin{aligned}
& \varphi=\operatorname{sign}\left(\sigma_{12} \cos \phi+\sigma_{13} \sin \phi\right) \varphi_{0}+ \\
& \frac{\left(\sigma_{22} \cos ^{2} \phi+\sigma_{33} \sin ^{2} \phi+\sigma_{23} \sin 2 \phi-\sigma_{11}\right) \varphi+\left(\sigma_{12} \cos \phi+\sigma_{13} \sin \phi\right)}{G_{12}}
\end{aligned}
$$

$\mathrm{ou}$

$$
\varphi=\frac{\operatorname{sign}\left(\sigma_{12} \cos \phi+\sigma_{13} \sin \phi\right) G_{12} \varphi_{0}+\left(\sigma_{12} \cos \phi+\sigma_{13} \sin \phi\right)}{\left(\sigma_{11}-\sigma_{22} \cos ^{2} \phi-\sigma_{33} \sin ^{2} \phi-\sigma_{23} \sin 2 \phi+G_{12}\right)}
$$

Uma vez que todas as quantidades necessárias para formular o critério são obtidas, o mesmo já está plenamente definido. Todavia, a implementação do cálculo das tensões atuantes no plano crítico para a fibra sob compressão podem ser expressas de formas mais simples. Relembrando, as tensões em notação tensorial são escritas como

$$
\sigma_{i j}^{(m i s)}=\lambda_{i k} \lambda_{j l} \sigma_{k l}^{(\phi)}=\lambda_{i k} \lambda_{j l} \lambda_{k m} \lambda_{l n} \sigma_{m n}
$$

Como é necessária a rotação em dois planos diferentes, pode-se simplificar a equação escrevendo a mesma em notação matricial

$$
\boldsymbol{\sigma}^{(m i s)}=\boldsymbol{\Omega} \boldsymbol{\sigma} \boldsymbol{\Omega}^{T}
$$

onde a matriz de rotação é definida como (RAND; ROVENSKI, 2005)

$$
\begin{aligned}
& \boldsymbol{\Omega}=\boldsymbol{\Omega}_{\varphi_{0}}^{(12)} \boldsymbol{\Omega}_{\phi}^{(23)} \\
& \boldsymbol{\Omega}_{\varphi}^{(12)}=\left[\begin{array}{ccc}
\cos \varphi & \sin \varphi & 0 \\
-\sin \varphi & \cos \varphi & 0 \\
0 & 0 & 1
\end{array}\right]
\end{aligned}
$$




$$
\boldsymbol{\Omega}_{\phi}^{(23)}=\left[\begin{array}{ccc}
1 & 0 & 0 \\
0 & \cos \phi & \sin \phi \\
0 & -\sin \phi & \cos \phi
\end{array}\right]
$$

\subsubsection{Tensão Plana}

Considerando o caso de tensão plana, a única função de falha que possui solução analítica explícita é a que modela a falha na fibra por tração, que pode ser reescrita exatamente como a equação (119), ou seja,

$$
f_{L}^{(f, t)}=\frac{\sigma_{11}}{S_{11}^{t}}
$$

Para a falha da matriz, pode-se escrever

$$
f_{L}^{(m)}=\left(\frac{\sigma_{12} \cos \gamma}{S_{12}-b_{L} \sigma_{22} \cos ^{2} \gamma}\right)^{2}+\left(\frac{\sigma_{22} \sin \gamma \cos \gamma}{S_{23}^{(23)}-b_{T} \sigma_{22} \cos ^{2} \gamma}\right)^{2}+\left(\frac{\max \left\{0, \sigma_{22} \cos ^{2} \gamma\right\}}{S_{22}^{t}}\right)^{2}
$$

Sendo ainda necessário calcular o valor de $\gamma$ que maximiza a função, no entanto, para tensão plana, $0 \leq \gamma \leq 90^{\circ}$, o que diminui o custo computacional na busca pelo plano crítico.

A função de falha para fibra sob compressão é

$$
f_{L}^{(f, c)}=\left(\frac{\sigma_{12}^{(m i s)}}{S_{12}-b_{L} \sigma_{22}^{(m i s)}}\right)^{2}+\left(\frac{\sigma_{23}^{(m i s)}}{S_{23}^{(23)}-b_{T} \sigma_{22}^{(m i s)}}\right)^{2}+\left(\frac{\max \left\{0, \sigma_{22}^{(m i s)}\right\}}{S_{22}^{t}}\right)^{2}
$$

onde

$$
\begin{aligned}
& \sigma_{22}^{(m i s)}=\sigma_{11}^{(\phi)} \sin ^{2} \varphi+\sigma_{22}^{(\phi)} \cos ^{2} \varphi-2 \sigma_{12}^{(\phi)} \sin \varphi \cos \varphi \\
& \sigma_{12}^{(m i s)}=\left(\sigma_{22}^{(\phi)}-\sigma_{11}^{(\phi)}\right) \sin \varphi \cos \varphi+\sigma_{12}^{(\phi)}\left(\cos ^{2} \varphi-\sin ^{2} \varphi\right) \\
& \sigma_{23}^{(m i s)}=-\sigma_{13}^{(\phi)} \sin \varphi+\sigma_{23}^{(\phi)} \cos \varphi \\
& \mathrm{e} \\
& \sigma_{11}^{(\phi)}=\sigma_{11} \\
& \sigma_{22}^{(\phi)}=\sigma_{22} \cos ^{2} \phi \\
& \sigma_{12}^{(\phi)}=\sigma_{12} \cos \phi
\end{aligned}
$$




$$
\begin{aligned}
& \sigma_{13}^{(\phi)}=-\sigma_{12} \sin \phi \\
& \sigma_{23}^{(\phi)}=-\sigma_{22} \sin \phi \cos \phi
\end{aligned}
$$
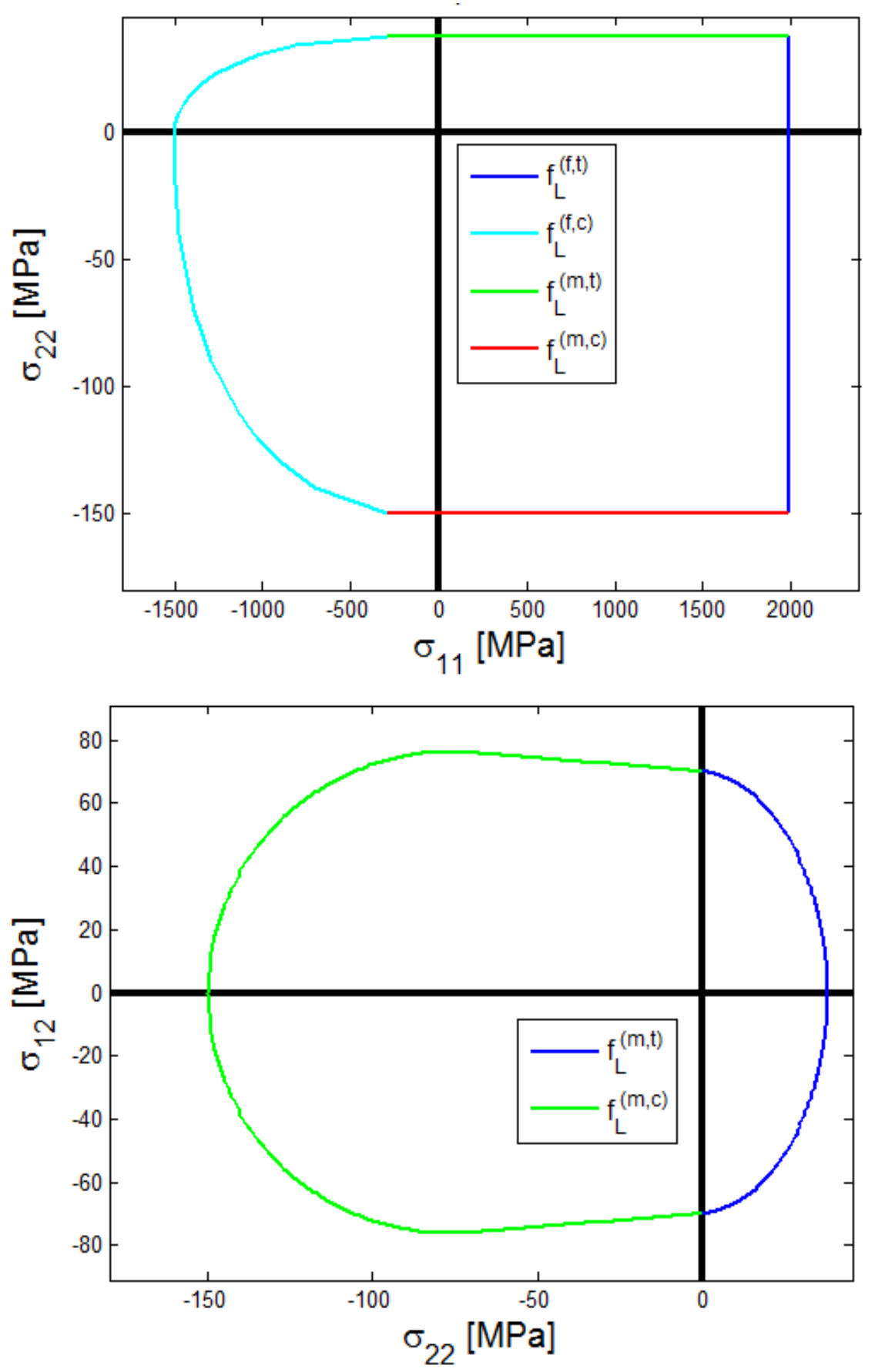

Figura 15 - Principais envelopes de falha pelo modelo de LaRC05 

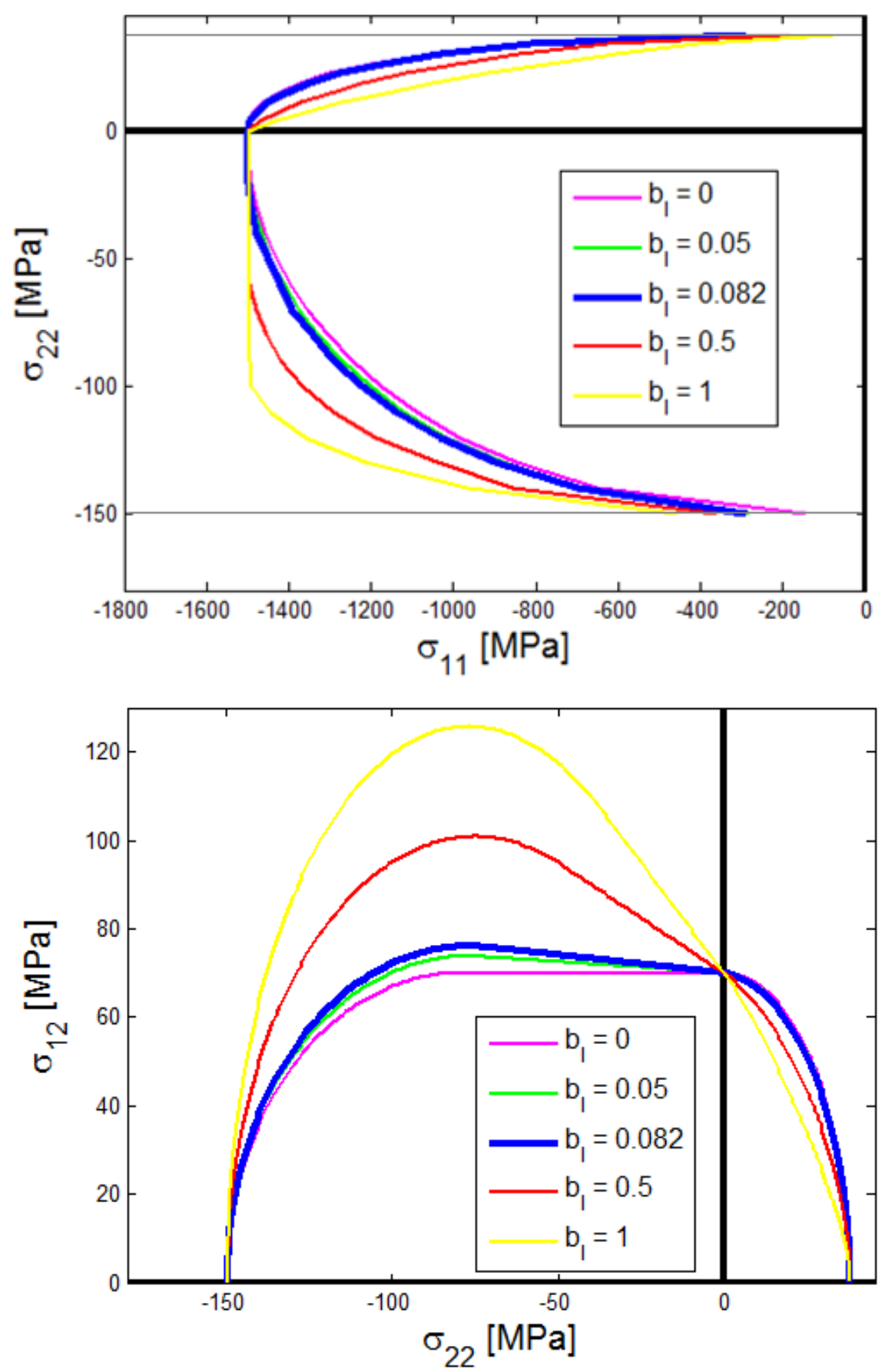

Figura 16 - Influência do parâmetro $b_{l}$ no envelope de falha de LaRC05

Explicitando as equações (145.a-c) em função do carregamento utilizando as equações (146.a-e), tem-se

$$
\sigma_{22}^{(m i s)}=\sigma_{11} \sin ^{2} \varphi+\sigma_{22} \cos ^{2} \phi \cos ^{2} \varphi-2 \sigma_{12} \cos \phi \sin \varphi \cos \varphi
$$




$$
\begin{aligned}
& \sigma_{12}^{(m i s)}=\left(\sigma_{22} \cos ^{2} \phi-\sigma_{11}\right) \sin \varphi \cos \varphi+\sigma_{12} \cos \phi\left(\cos ^{2} \varphi-\sin ^{2} \varphi\right) \\
& \sigma_{23}^{(m i s)}=\sigma_{12} \sin \phi \sin \varphi-\sigma_{22} \sin \phi \cos \phi \cos \varphi
\end{aligned}
$$

E a definição do ângulo $\varphi$ pode ser simplificada para

$$
\varphi=\frac{\operatorname{sign}\left(\sigma_{12} \cos \phi\right) G_{12} \varphi_{0}+\left(\sigma_{12} \cos \phi\right)}{\left(\sigma_{11}-\sigma_{22} \cos ^{2} \phi+G_{12}\right)}
$$

Uma discussão para casos de tensão plana pode ser encontrada em Dávila e Camanho (2003).

Utilizando as equações anteriores pode-se, então, obter os envelopes de falha nos planos $\sigma_{11}-\sigma_{22}$ e $\sigma_{22}-\sigma_{12}$ como mostrados nas Figuras 15 .

A influência do parâmetro ajustável $b_{l}$ se torna clara nas Figuras 16.

O significado físico dos parâmetros $\theta_{0}$ e $b_{T}$ podem ser entendidos pelo círculo de Mohr, como mostrado por Pinho et al. (2005). Considerando o caso de compressão uniaxial na direção perpendicular às fibras, o círculo de Mohr no plano $x_{2}^{(23)}-x_{3}^{(23)}$ está representado pela Figura 17 e as componentes de tensões podem ser escritas como (CRANDALL et al., 1978)

$$
\begin{aligned}
& \sigma_{22}^{(23)}=-\frac{S_{22}^{c}}{2}(1+\cos 2 \theta) \\
& \sigma_{23}^{(23)}=\frac{S_{22}^{c}}{2} \sin 2 \theta
\end{aligned}
$$

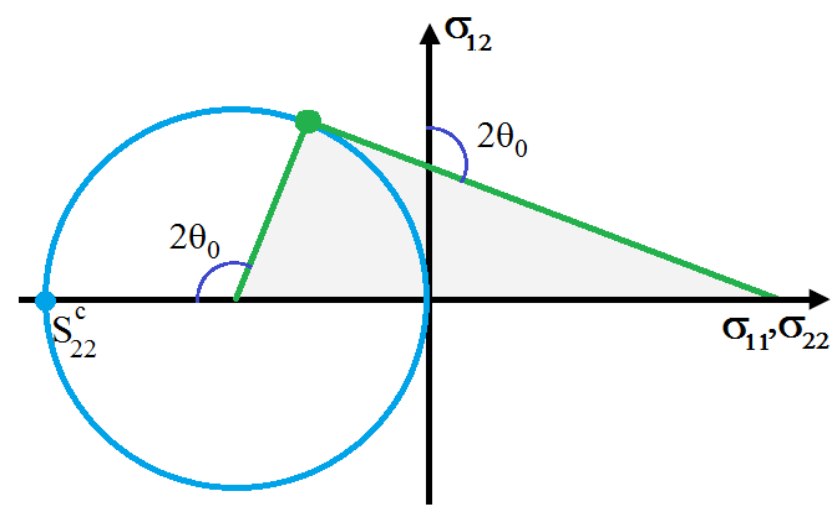

Figura 17 - Círculo de Mohr para compressão uniaxial perpendicular às fibras

Assumindo um critério de falha para a matriz do tipo Mohr-Coulomb, pode-se escrever

$$
\sigma_{23}^{(23)}+b_{T} \sigma_{22}^{(23)}=S_{23}^{(23)}
$$


onde, por definição,

$b_{T}=-\frac{1}{\tan 2 \theta_{0}}$

E substituindo as equações (149) e (151) na equação (150)

$$
S_{23}^{(23)}=S_{22}^{c} \cos \theta_{0}\left(\sin \theta_{0}+\frac{\cos \theta_{0}}{\tan 2 \theta_{0}}\right)
$$

Note que esse procedimento deduz as equações (118.a,b) - as mesmas são iguais à (151) e (152) - e que o critério de Mohr-Coulomb mostrado na equação (150) é uma caso específico da equação (117) que define a falha da matriz.

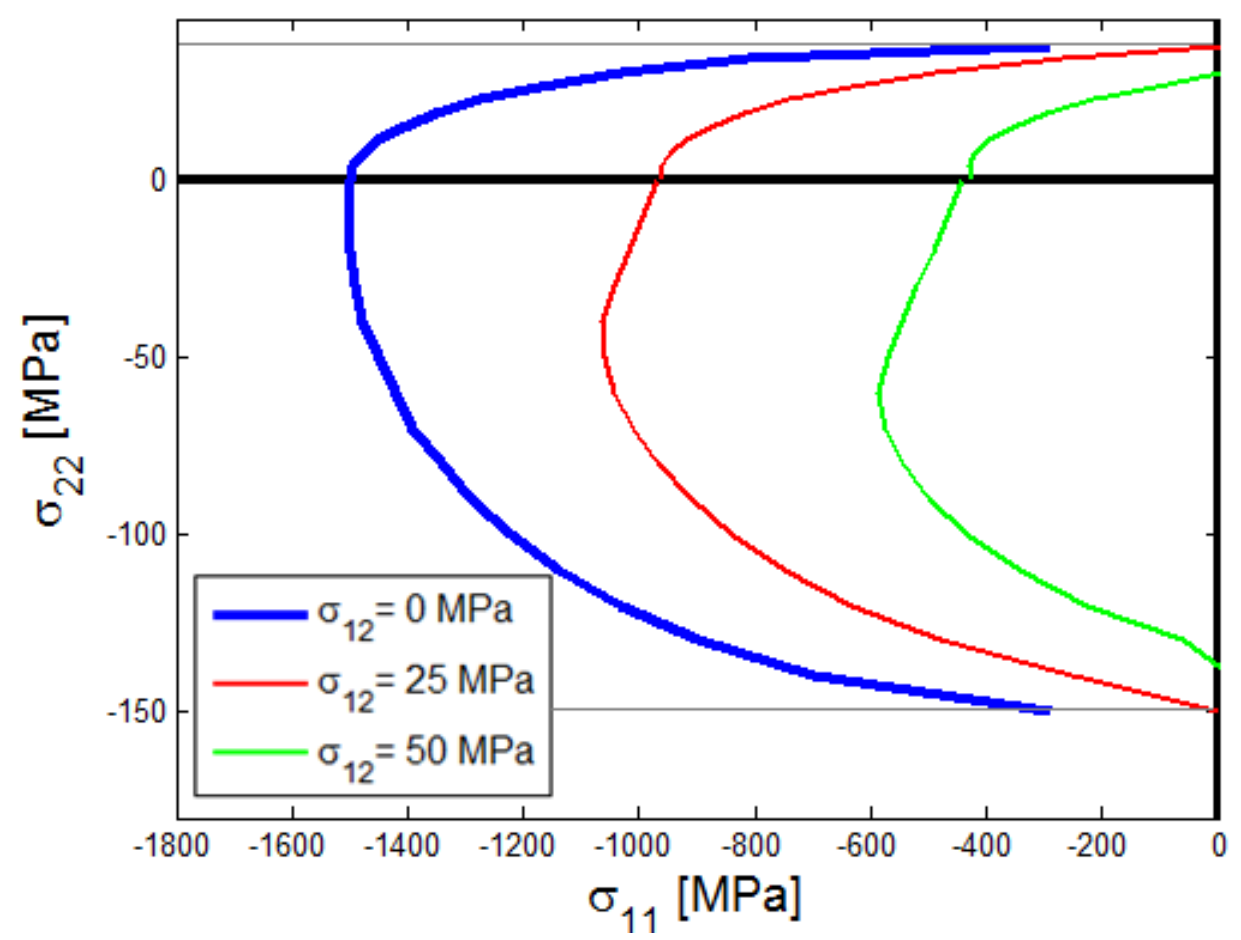

Figura 18 - Efeito da terceira componente de tensão (para tensão plana) no envelope de falha LaRC05

O efeito da terceira componente da tensão, para o caso de tensão plana, só precisa ser avaliado para o envelope $\sigma_{11}-\sigma_{22}$ (Figura 18), visto que os modelos de falha da matriz e da fibra sob tração não são influenciados pelas componentes $\sigma_{11}$ e $\sigma_{12}$, respectivamente, nem para o caso genérico 3D - ver equações (117) e (119). 


\subsection{Comparação entre os envelopes de falha}

Para uma melhor avaliação dos envelopes de falha mostrados anteriormente e uma comparação direta entre os modelos, na Figura 19 estão mostrados os envelopes de falha sobrepostos.
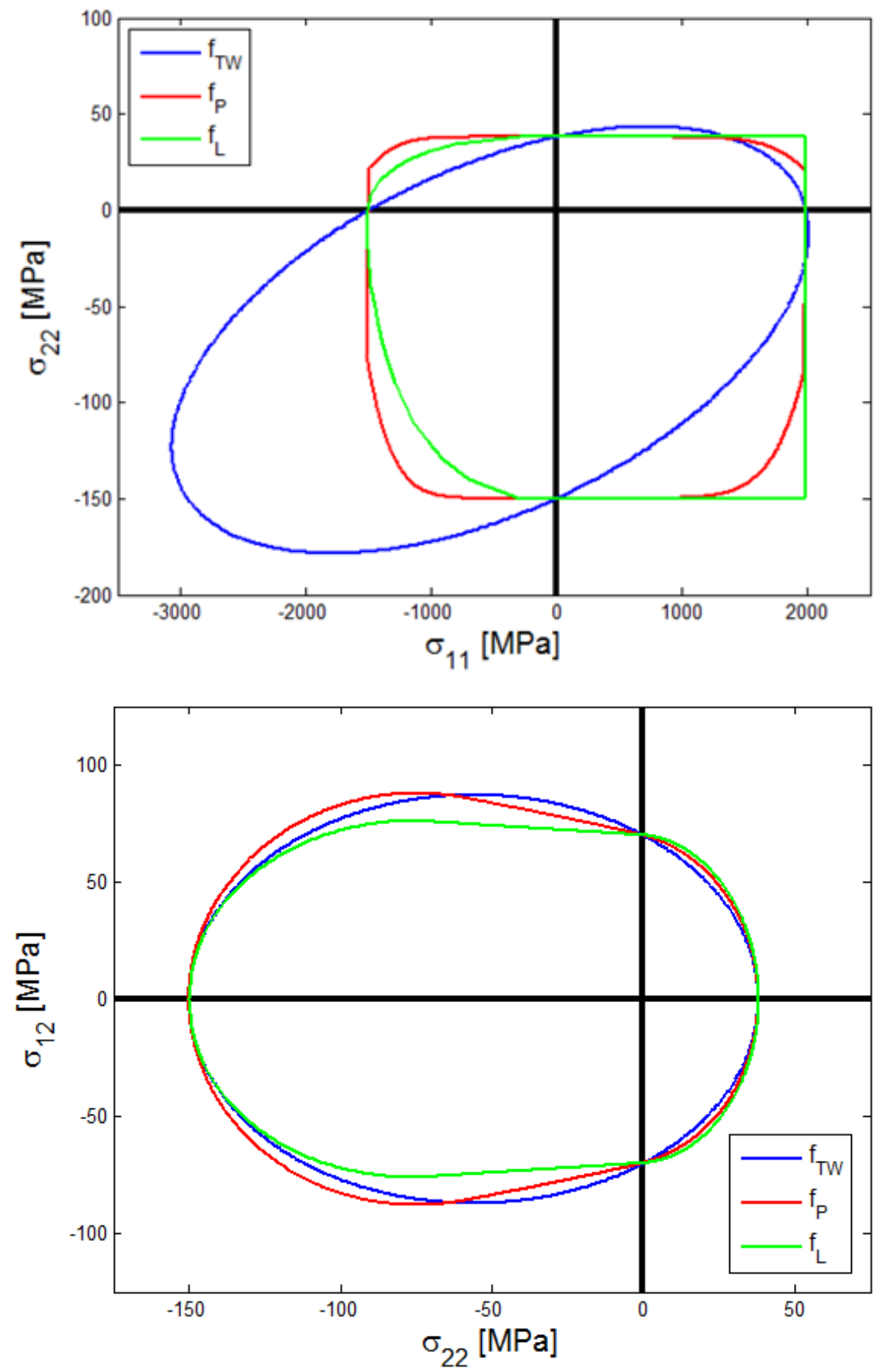

Figura 19 - Comparação entre os envelopes de falha 
Pode-se perceber que a predição de falha na matriz é bem similar em todos os modelos. Esse fato pode ser explicado porque o modelo LaRC05 usa a teoria do modelo de Puck como base, principalmente, para a modelagem da matriz, enquanto o modelo de Tsai$\mathrm{Wu}$, que é um ajuste de dados experimentais, também tem uma forma bem similar. A equação do modelo de Tsai-Wu é um ajuste polinomial e é natural que a mesma se adeque melhor à modelagem da matriz uma vez que a quantidade de dados experimentais desse tipo de falha é mais vasto na literatura, todavia, por considerar a interação entre as componentes de tensão, quando $\sigma_{11} \neq 0$ o envelope de falha se torna consideravelmente diferente dos outros.

Por outro lado, no plano $\sigma_{11}-\sigma_{22}$ a diferença entre as predições se tornam mais claras, principalmente do modelo de Tsai-Wu. Como comentado anteriormente, o mecanismo de falha da fibra sob compressão é o mais difícil de ser modelado, e como esperado, é onde as diferenças se tornam mais significativas.

\subsection{Estimativa da resistência de laminados}

A TCL mostrada no capítulo 2 pode ser aplicada a qualquer tipo de laminado, independente de qualquer simetria. Entretanto, para se obter uma comparação direta entre os resultados obtidos para lâminas, os laminados classificados como angle-ply, ou seja, $[ \pm \alpha]_{s}$ serão estudados.

A matriz de rigidez de cada camada pode ser obtida em coordenadas globais como

$$
s_{i j k n}^{(g)}=\lambda_{i o} \lambda_{j p} \lambda_{k q} \lambda_{n r} s_{o p q r}^{(l)}
$$

onde o ângulo entre $x_{i}^{(l)}$ e $x_{i}^{(g)}$ é $\alpha$, mas a rotação é realizada no sentido trigonométrico, por exemplo, para as camadas $+\alpha$, a rotação é $-\alpha$. Considerando que todas as lâminas têm a espessura de $1 \mathrm{~mm}$, por simplicidade, e que deseja-se apenas estudar os casos de tração e compressão uniaxial, os elementos das matrizes $\boldsymbol{A}$ e $\boldsymbol{B}$ podem ser escritos como

$$
\begin{aligned}
& A_{i j k n}^{(g)}=\left[s_{i j k n}^{(g)}\right]_{+\alpha}+\left[s_{i j k n}^{(g)}\right]_{-\alpha}+\left[s_{i j k n}^{(g)}\right]_{-\alpha}+\left[s_{i j k n}^{(g)}\right]_{+\alpha}=2\left(\left[s_{i j k n}^{(g)}\right]_{+\alpha}+\left[s_{i j k n}^{(g)}\right]_{-\alpha}\right) \\
& B_{i j k l}^{(g)}=\frac{1}{2}\left(\left[s_{i j k l}^{(g)}\right]_{+\alpha}(1-4)+\left[s_{i j k l}^{(g)}\right]_{-\alpha}(0-1)+\left[s_{i j k l}^{(g)}\right]_{-\alpha}(1-0)+\left[s_{i j k l}^{(g)}\right]_{+\alpha}(4-1)\right)=0
\end{aligned}
$$


É importante ressaltar que considerar a espessura de $1 \mathrm{~mm}$ não perde a generalidade da análise, apenas fica mais claro o resultado. Então, cada elemento da matriz $\boldsymbol{A}$ pode ser explicitado como (note que a mesma é simétrica)

$$
\begin{aligned}
& A_{1111}^{(g)}=2\left(\left[s_{1111}^{(g)}\right]_{+\alpha}+\left[s_{1111}^{(g)}\right]_{-\alpha}\right) \\
& A_{1122}^{(g)}=2\left(\left[s_{1122}^{(g)}\right]_{+\alpha}+\left[s_{1122}^{(g)}\right]_{-\alpha}\right) \\
& A_{1112}^{(g)}=2\left(\left[s_{1112}^{(g)}\right]_{+\alpha}+\left[s_{1112}^{(g)}\right]_{-\alpha}\right) \\
& A_{2222}^{(g)}=2\left(\left[s_{2222}^{(g)}\right]_{+\alpha}+\left[s_{2222}^{(g)}\right]_{-\alpha}\right) \\
& A_{2212}^{(g)}=2\left(\left[s_{2212}^{(g)}\right]_{+\alpha}+\left[s_{2212}^{(g)}\right]_{-\alpha}\right) \\
& A_{1212}^{(g)}=2\left(\left[s_{1212}^{(g)}\right]_{+\alpha}+\left[s_{1212}^{(g)}\right]_{-\alpha}\right)
\end{aligned}
$$

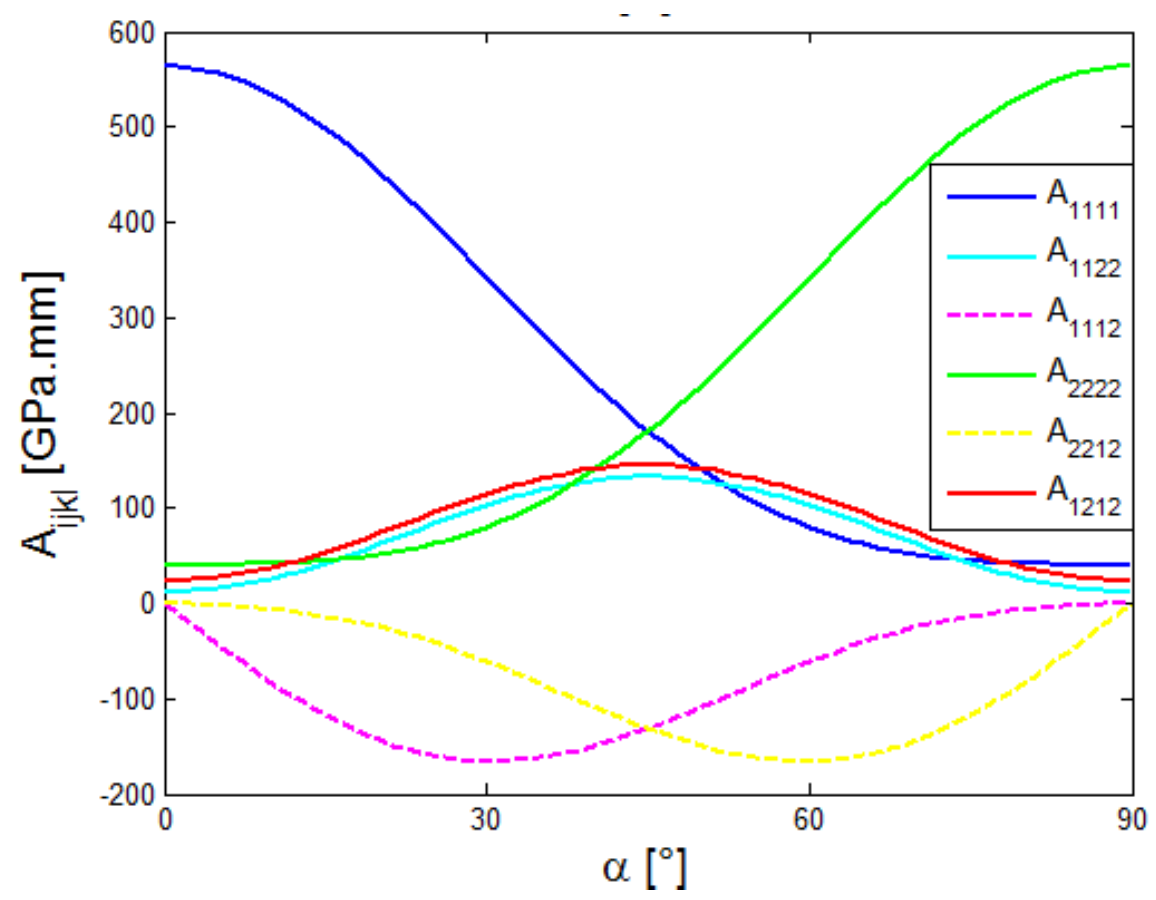

Figura 20 - Variação das propriedades dos laminados $[\alpha]_{n}$ e $[ \pm \alpha]_{n s}$

Utilizando as propriedades mostradas na Tabela 1, a variação dos valores dos elementos da matriz $A$ de acordo com $\alpha$ estão mostrados na Figura 20. É importante 
ressaltar que os elementos $A_{1111}, A_{1122}, A_{2222}$ e $A_{1212}$ são iguais para os laminados $[\alpha]_{n}$ e $[ \pm \alpha]_{n s}$ e os elementos $A_{1112}$ e $A_{2212}$ são nulos para $[ \pm \alpha]_{n s}$ e estão representados pelas linhas pontilhadas para $[\alpha]_{n}$. Note que os únicos elementos diferentes são os responsáveis pelo acoplamento entre as tensões e deformações normais e cisalhantes.

Para calcular o efeito do carregamento, com base na teoria desenvolvida no capítulo 2, pode-se seguir o seguinte procedimento:

i. considera-se a aplicação de uma tensão no $\tilde{\boldsymbol{\sigma}}^{(g)}$ laminado, que resulta em uma força por unidade de espessura $\boldsymbol{N}=\tilde{\boldsymbol{\sigma}}^{(g)} t$, onde $t$ é a espessura do total;

ii. pode-se então calcular a deformação resultante usando $\varepsilon=A^{-1} \boldsymbol{N}$;

iii. uma vez que as deformações são obtidas e as mesmas são contínuas, ou seja, a deformação de todos as lâminas são iguais para o carregamento de forças, a tensão na lâmina $k$ pode ser calculada como $\left[\boldsymbol{\sigma}^{(g)}\right]_{k}=\left[\boldsymbol{s}^{(g)}\right]_{k} \boldsymbol{\varepsilon}$;

iv. as tensões em cada lâmina podem ser, então, computadas em coordenadas locais, de acordo com a orientação das fibras em cada lâmina como $\sigma_{i j}^{(l)}=\lambda_{i r} \lambda_{j s} \sigma_{r s}^{(g)}$

v. por último, o dano para um dado carregamento pode ser calculado substituindo as tensões em cada lâmina em coordenadas locais nas funções que definem os critérios de falha.

Dessa forma, pode-se estimar a resistência dos laminados $[\alpha]_{n}$ e $[ \pm \alpha]_{n s}$ em comparação com a resistência da lâmina, como mostrado na Figura 21. Torna-se válido então definir a resistência que será utilizada como referência para todos os resultados nesse trabalho: define-se $S_{F P F}$ o valor da tensão nominal (aplicada) necessária para início do dano, podendo haver um índice superior para definir o tipo de carregamento (e.g. os índices superiores $t$ e $c$ na Figura 21 indicam tração e compressão, respectivamente).

Segundo Pinho et al. (2005), a estimativa usando o critério LaRC05 é compatível com dados experimentais para compressão e a justificativa da diferença do resultado do mesmo com outros critérios fenomenológicos, como por exemplo o de Puck, é a consideração do desalinhamento inicial das fibras. 

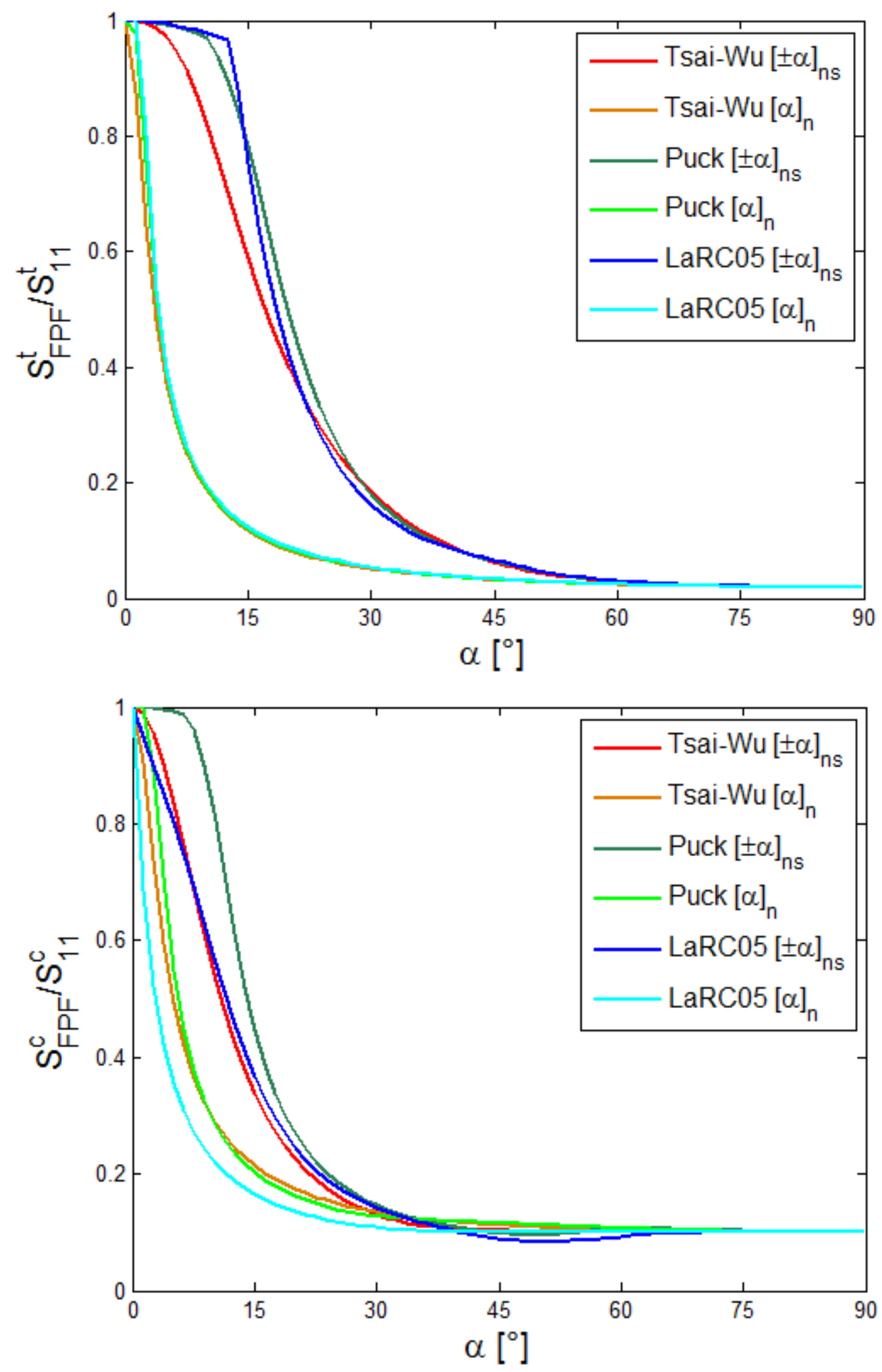

Figura 21 - Comparação da estimativa da resistência à tração e à compressão de laminados

$$
[\alpha]_{n} \text { e }[ \pm \alpha]_{n s}
$$


A Figura 21 mostra a vantagem na utilização dos laminados $[ \pm \alpha]_{n s}$ para a maioria dos valores de $\alpha$. Embora apenas o caso de carregamento uniaxial tenha sido mostrado, o resultado pode ser expandido para carregamentos genéricos. 


\section{Efeito de furos em placas sob tensão plana}

Na maioria dos casos práticos, adota-se a hipótese de tensão plana na modelagem de laminados tanto para abordagens analíticas, pois casos 3D nem sempre têm soluções conhecidas, quanto para métodos numéricos, para diminuir o custo computacional.

Antes de avaliar o efeito de um furo na resistência de uma placa torna-se útil uma comparação entre problemas de concentração de tensões entre materiais isotrópicos e anisotrópicos. Furos circulares em placas isotrópicas são devidamente estudados no domínio $0^{\circ} \leq \theta \leq 90^{\circ}$, onde $\theta$ é a posição angular na borda do furo. Todavia, a simetria de um quarto não é aplicável para materiais anisotrópicos por causa da periodicidade da solução da distribuição de tensão. Na verdade, a utilização de condições de contorno em modelos numéricos deve ser evitada para materiais anisotrópicos mesmo quando a periodicidade da solução é respeitada. A Figura 22 mostra um exemplo importante de erro conceitual gerado pela aplicação de simetria. Supondo que a utilização de da simetria seja aplicável, a representação física da geometria completa resulta em uma não continuidade do campo de deformações, consequentemente não respeita a condição de compatibilidade geométrica, uma vez que a taxa de variação do campo de deslocamentos não pode ser contínua. Posteriormente, no estudo de placas finitas pelo MEF, essa conclusão será utilizada.

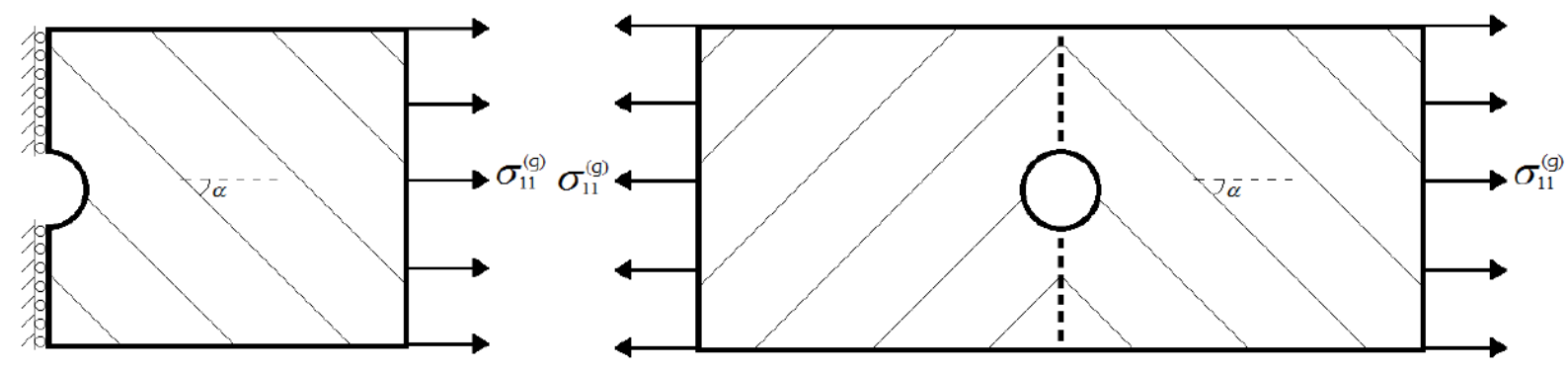

Figura 22 - Ilustração do efeito da condição de simetria

Os efeitos de concentração de tensão induzidos por um furo circular em uma placa muito grande de um laminado $[\alpha]_{n}$, com os ângulos das fibras iguais a $\alpha=0^{\circ}, 15^{\circ}, \ldots, 90^{\circ}$ ou $\alpha=-60^{\circ},-45^{\circ}, \ldots, 90^{\circ}$, dependendo do carregamento, são avaliados na Figura 23 para alguns importantes casos. 

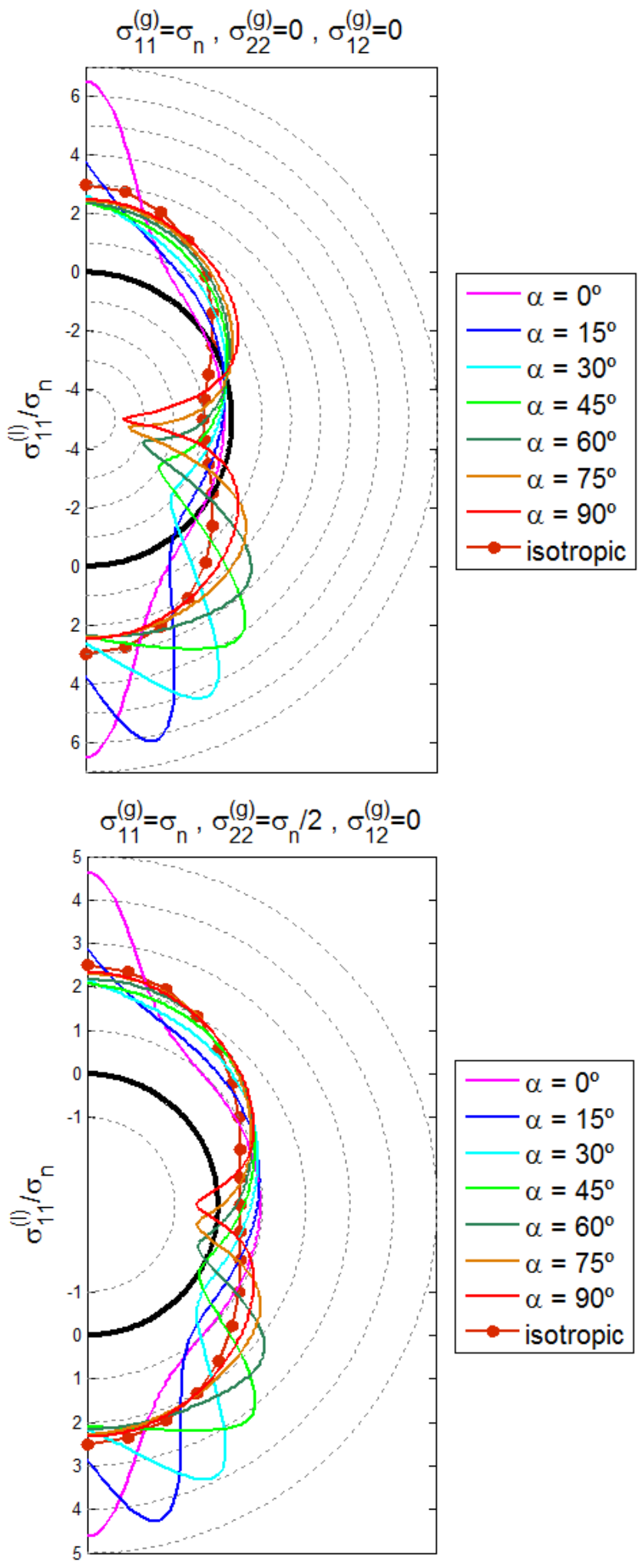

Figura 23 - Razão entre a distribuição da tensão tangencial na borda do furo e a tensão nominal $\left(\sigma_{11}^{(l)} / \sigma_{n}\right.$ ) para uma placa com furo circular e diversos valores de $\alpha$ sob diferentes condições de carregamento (continua) 


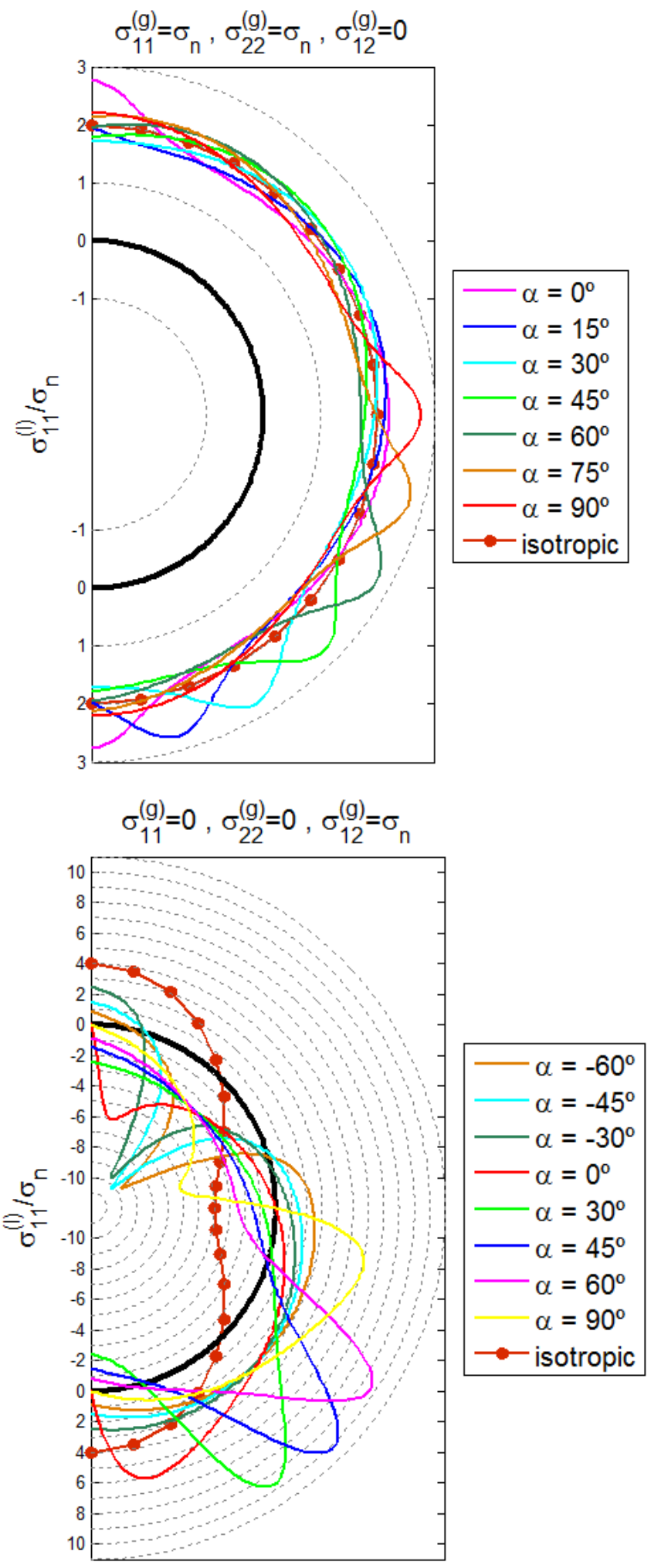

Figura 23 - Razão entre a distribuição da tensão tangencial na borda do furo e a tensão nominal $\left(\sigma_{11}^{(l)} / \sigma_{n}\right.$ ) para uma placa com furo circular e diversos valores de $\alpha$ sob diferentes condições de carregamento (continua) 


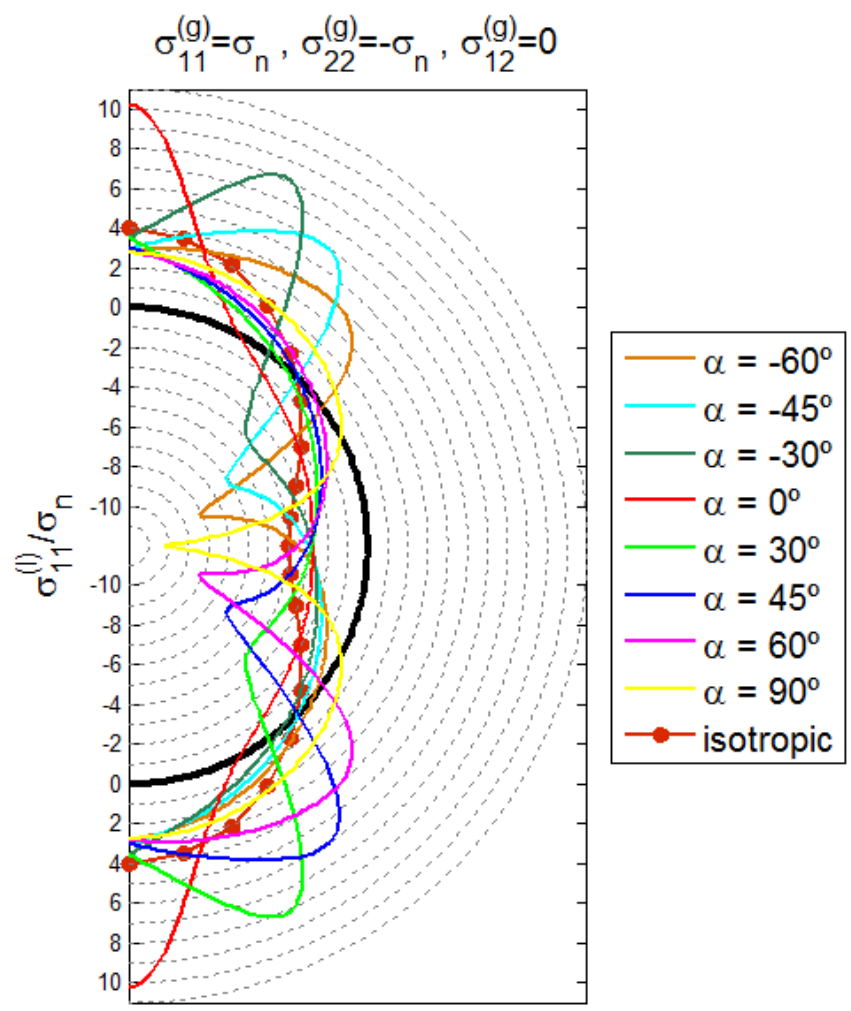

Figura 23 - Razão entre a distribuição da tensão tangencial na borda do furo e a tensão nominal $\left(\sigma_{11}^{(l)} / \sigma_{n}\right)$ para uma placa com furo circular e diversos valores de $\alpha$ sob diferentes condições de carregamento (continuação)

Comparando esses resultados com os equivalentes para materiais isotrópicos, que estão sobrepostos no gráfico, as seguintes conclusões podem ser postas:

i. para uma tensão nominal uniaxial $\sigma_{11}^{(g)}=\sigma_{n}$ (aplicada na direção horizontal da figura), a distribuição da tensão tangencial ao longo da borda do furo varia de $-4 \sigma_{n}$ até quase $7 \sigma_{n}$, enquanto para materiais isotrópicos a faixa de valores é de $-1 \sigma_{n}$ até $3 \sigma_{n}$;

ii. para a solução clássica de vasos de pressão cilíndricos com paredes finas, $\sigma_{11}^{(g)}=\sigma_{22}^{(g)} / 2=\sigma_{n}$, a tensão tangente na borda do furo pode ser maior do que $4 \sigma_{n}$ e também pode ser compressiva, enquanto para materiais isotrópicos a mesma é sempre trativa e varia entre $0.5 \sigma_{n}$ e $2.5 \sigma_{n}$; 
iii. para o carregamento biaxial $\sigma_{11}^{(g)}=\sigma_{22}^{(g)}=\sigma_{n}$ há uma pequena variação na distribuição da tensão tangencial para o caso anisotrópico enquanto para o isotrópico é constante e igual a $2 \sigma_{n}$;

iv. para materiais isotrópicos, os carregamentos biaxial de tensão/compressão $\sigma_{11}^{(g)}=-\sigma_{22}^{(g)}=\sigma_{n}$ e cisalhamento puro $\sigma_{12}^{(g)}=\sigma_{n}$ são equivalentes e induzem um concentração de tensão de $4 \sigma_{n}$, enquanto para materiais anisotrópicos o efeito desses dois carregamentos são significativamente diferentes, como mostrado nas figuras anteriores, por causa da não-simetria gerada pelas fibras, sendo um resultado não intuitivo a priori.
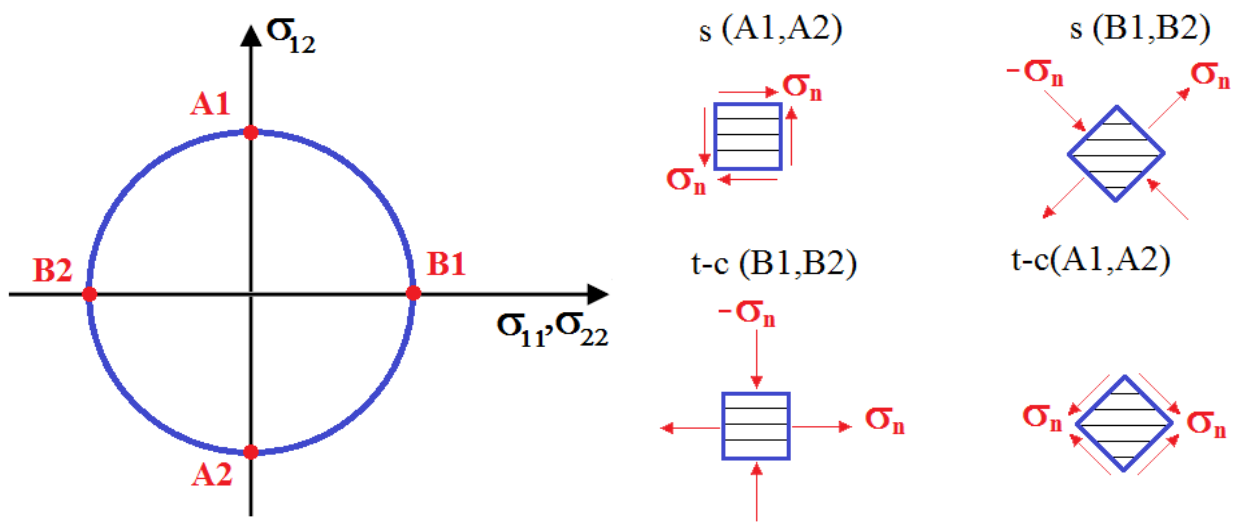

$\mathrm{t}-\mathrm{c}(\mathrm{A} 1, \mathrm{~A} 2)$
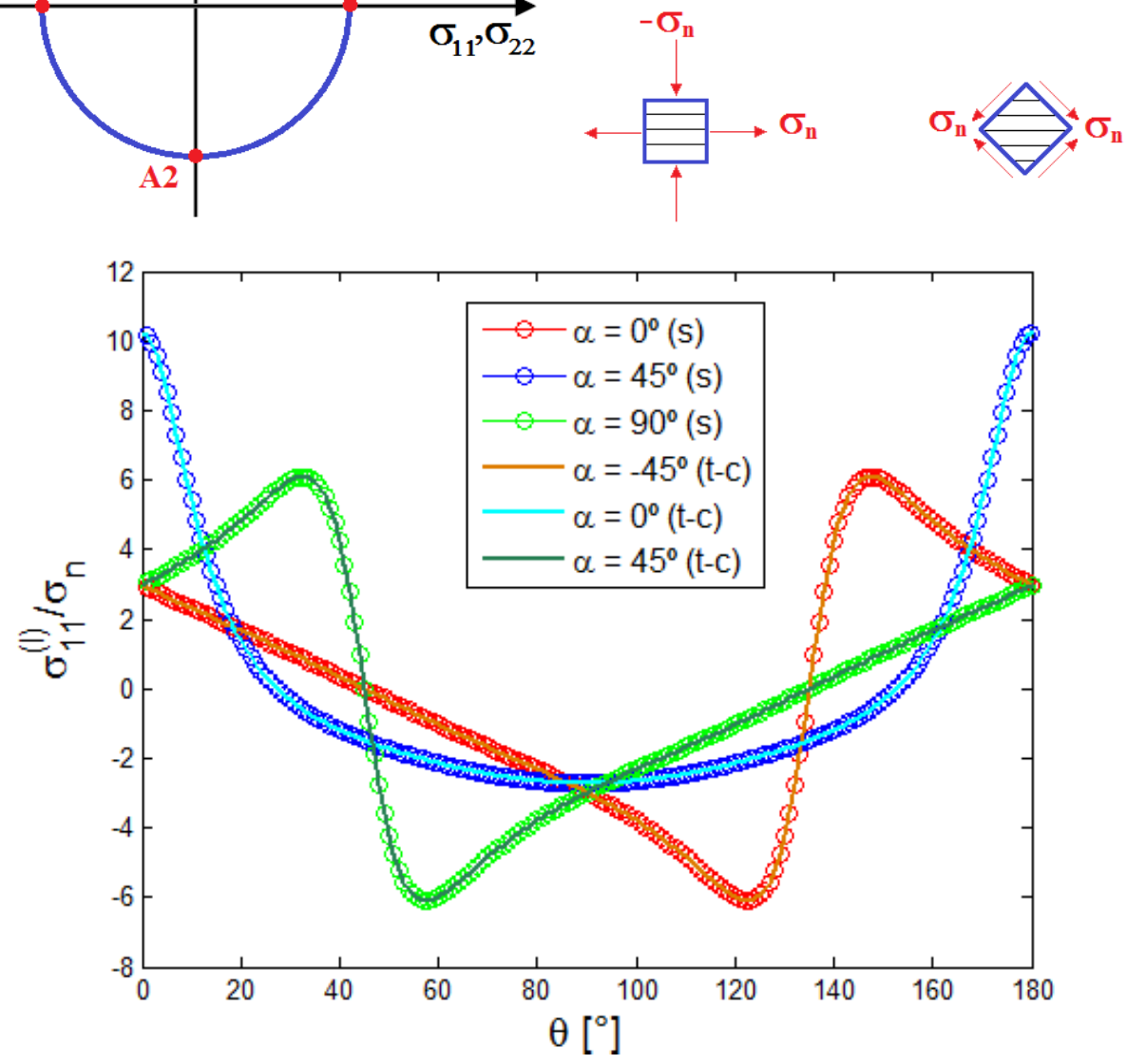

Figura 24 - Diferença entre os carregamentos de cisalhamento puro e tração/compressão 
A diferença entre o cisalhamento puro (s) e tensão/compressão (t-c) é devida à influência da direção das fibras. A Figura 24 mostra o círculo de Mohr, que é igual para ambos os casos e um elemento diferencial, considerando $\alpha=0^{\circ}$ por simplicidade. A concentração de tensão é similar para ambos, como deveria ser, mas precisa ser considerada uma rotação de $45^{\circ}$. Em outras palavras, a resistência ao cisalhamento pode ser obtida experimentalmente em um teste de tensão/compressão, mas a resistência obtida é referente a fibras com direções diferentes.

\subsection{Estimativas de resistências para laminados $[\alpha]_{n}$ com furo circular}

Para aplicar os critérios de falha vistos anteriormente é necessário transformar as tensões obtidas de coordenadas locais para coordenadas do material. Para carregamentos uniaxiais, as concentrações de tensões em coordenadas do material são mostradas na Figura 25 para vários valores de $\alpha$, de acordo com o ângulo $\theta$ que mapeia a borda do furo. Estimativas de resistências utilizando esses utilizando os três critérios de falha estudados são mostrados na Figura 26 de acordo com o ângulo $\alpha$. Para tração, os modelos de falha chegaram a resultados parecidos, mas para compressão os resultados gerados foram significativamente diferentes. Os resultados foram normalizados em relação à maior resistência possível de acordo com o carregamento, o que para tração e compressão é equivalente a dizer que foi normalizando em relação às resistências obtidas na direção paralela às fibras. Note que, de acordo com a Tabela $1, S_{11}^{t} / S_{22}^{t}=52.4$ e $S_{11}^{c} / S_{22}^{c}=10$, ou seja, a falha da matriz tende a ser dominante. 

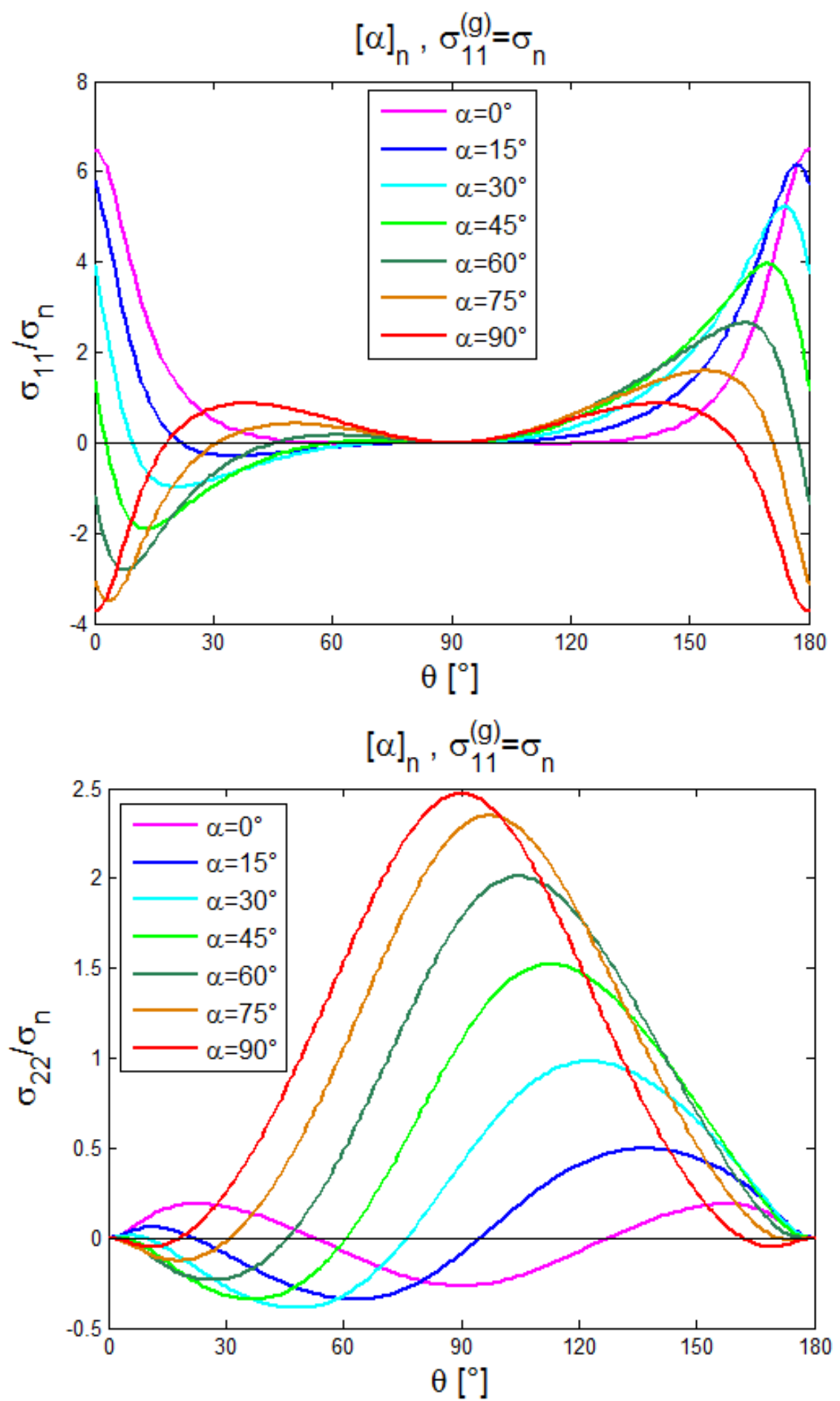

Figura 25 - Tensões ao longo da borda do furo em coordenadas do material para uma placa grande de laminado unidirecional com furo circular e carregada uniaxialmente (continua) 


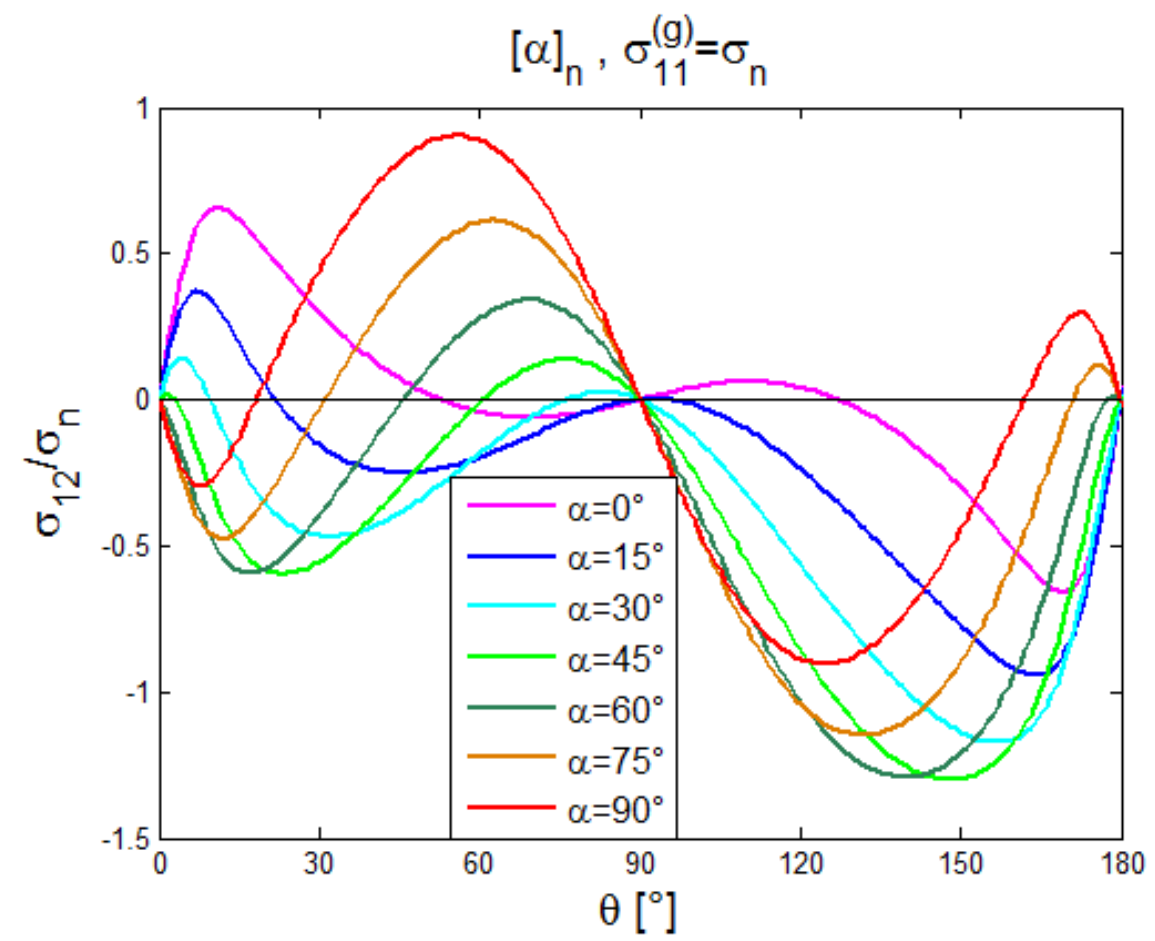

Figura 25 - Tensões ao longo da borda do furo em coordenadas do material para uma placa grande de laminado unidirecional com furo circular e carregada uniaxialmente (continuação)

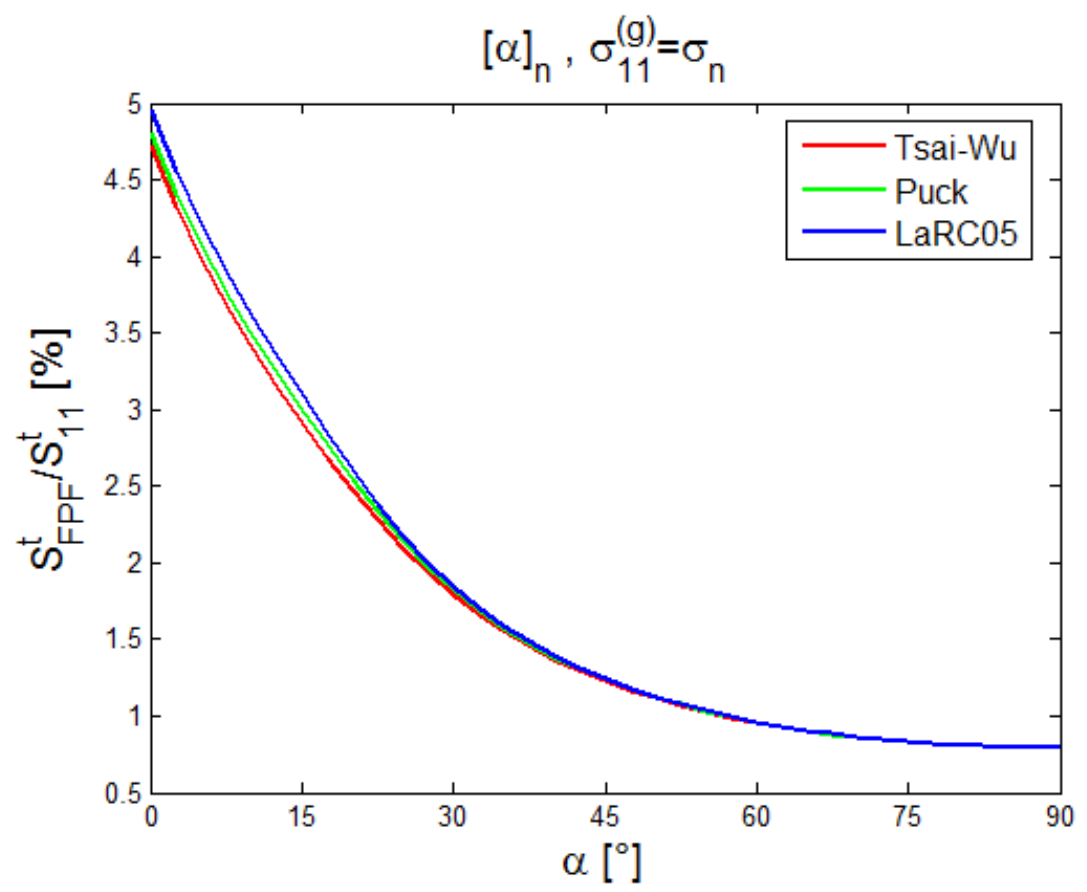

Figura 26 - Estimativas de $S_{F P F}^{t} / S_{11}^{t}$ e $S_{F P F}^{c} / S_{11}^{c}$ para placas grandes de laminados unidirecionais com furos circulares (continua) 


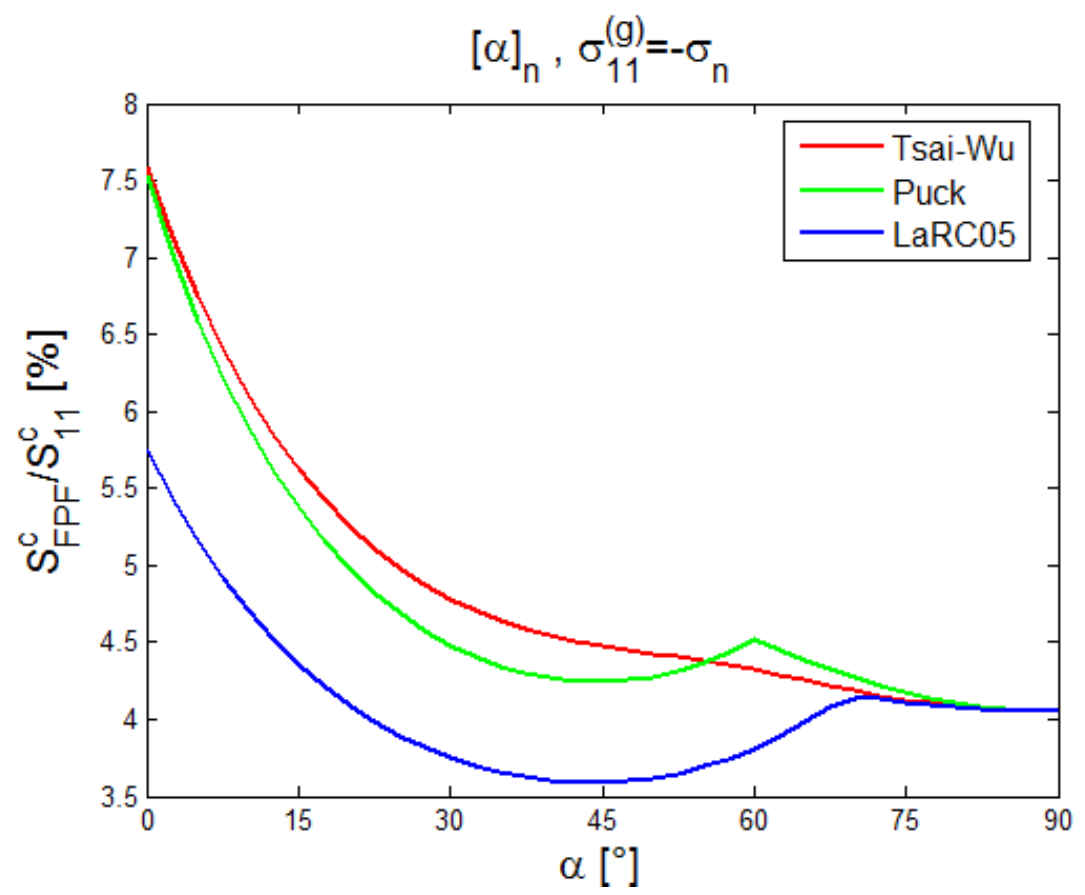

Figura 26 - Estimativas de $S_{F P F}^{t} / S_{11}^{t}$ e $S_{F P F}^{c} / S_{11}^{c}$ para placas grandes de laminados unidirecionais com furos circulares (continuação)

A seguir, três diferentes condições de carregamento são analisadas $\left(\sigma_{11}^{(g)}=90 M P a\right.$ e $\alpha=0^{\circ} ; \sigma_{11}^{(g)}=-70 M P a$ e $\alpha=15^{\circ}$; e $\sigma_{11}^{(g)}=-60 M P a$ e $\alpha=75^{\circ}$ ) para ilustrar a diferença entre as estimativas dos critérios de falha estudados.

Para carregamentos trativos, quando $\alpha=0^{\circ}$, é esperado que a falha se inicie quando $90 \mathrm{MPa}<\sigma_{11}^{(g)}<100 \mathrm{MPa}$ de acordo com todos os modelos. Como Puck e LaRC05 modelam a falha da matriz e de fibra de forma separada, a estimativa de falha para cada elemento é mostrada separadamente, e a falha da matriz é esperada para todos os casos. A Figura 27 mostra as estimativas para $\sigma_{11}^{(g)}=90 \mathrm{MPa}$ e $\alpha=0^{\circ}$; embora não haja a falha porque $f<1$, é possível avaliar o ponto crítico. Note que ao contrário do esperado para materiais isotrópicos, o ponto crítico é $\theta \cong 20^{\circ}, 160^{\circ}$ ao invés de $\theta=0^{\circ}$. 


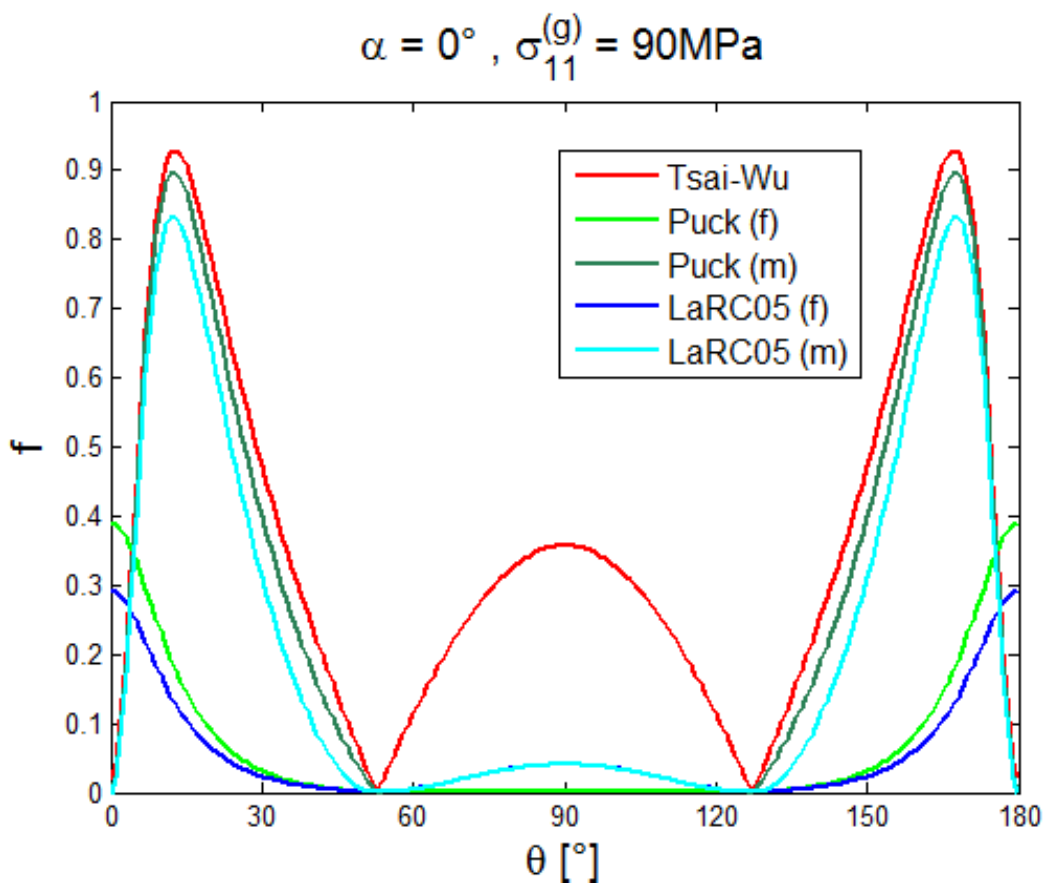

Figura 27 - Variação das funções de falha na borda do furo para o carregamento de tração uniaxial com $\sigma_{11}^{(g)}=90 M P a$ e $\alpha=0^{\circ}$ no laminado unidirecional

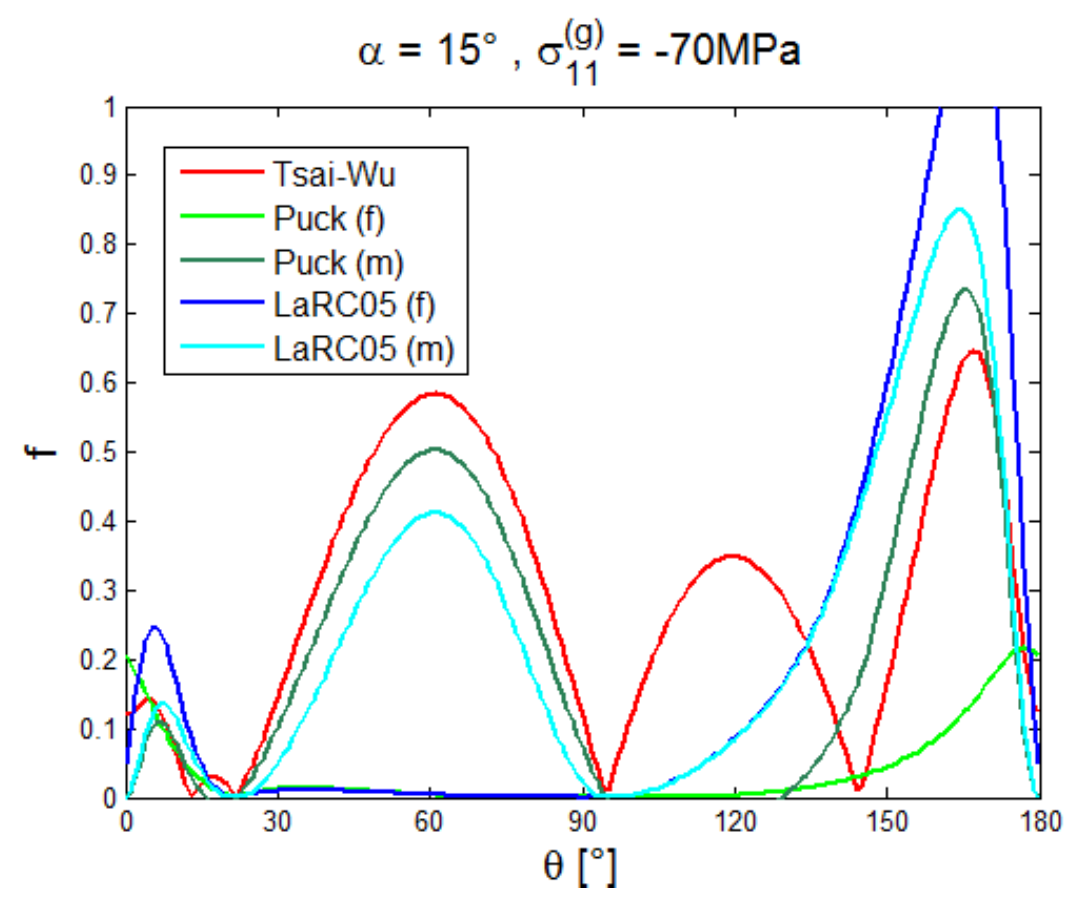

Figura 28 - Variação das funções de falha na borda do furo para o carregamento de compressão uniaxial com $\sigma_{11}^{(g)}=-70 M P a$ e $\alpha=15^{\circ}$ no laminado unidirecional 
A Figura 28 mostra a estimativa para uma placa com carregamento compressivo de -70MPa e ângulo $\alpha=15^{\circ}$. Nesse caso, a diferença entre os critérios de falha se torna mais significativa. Apenas o modelo LaRC05 indica a falha das fibras na posição $\theta \cong 170^{\circ}$, que é parecida, mas não coincide, com a posição crítica apontada pelos outros critérios, porém sem haver falha.

A Figura 29 mostra tendência das funções de falha para um carregamento compressivo de $-60 \mathrm{MPa}$ e $\alpha=75^{\circ}$. Novamente as estimativas entre os critérios se torna significativa. Nenhum modelo indica falha, mas todos eles indicam que o ponto crítico é $\theta \cong 100^{\circ}$, novamente também diferente do caso isotrópico. É interessante ressaltar que o modelo LaRC05 estima um tendência bastante parecida para a falha da matriz e da fibra, enquanto o modelo de Puck estima um valor muito menor da resistência da matriz. Esse caso poderia ser um bom teste para verificar qual dos critérios é o melhor para descrever os mecanismos relacionados à falha.

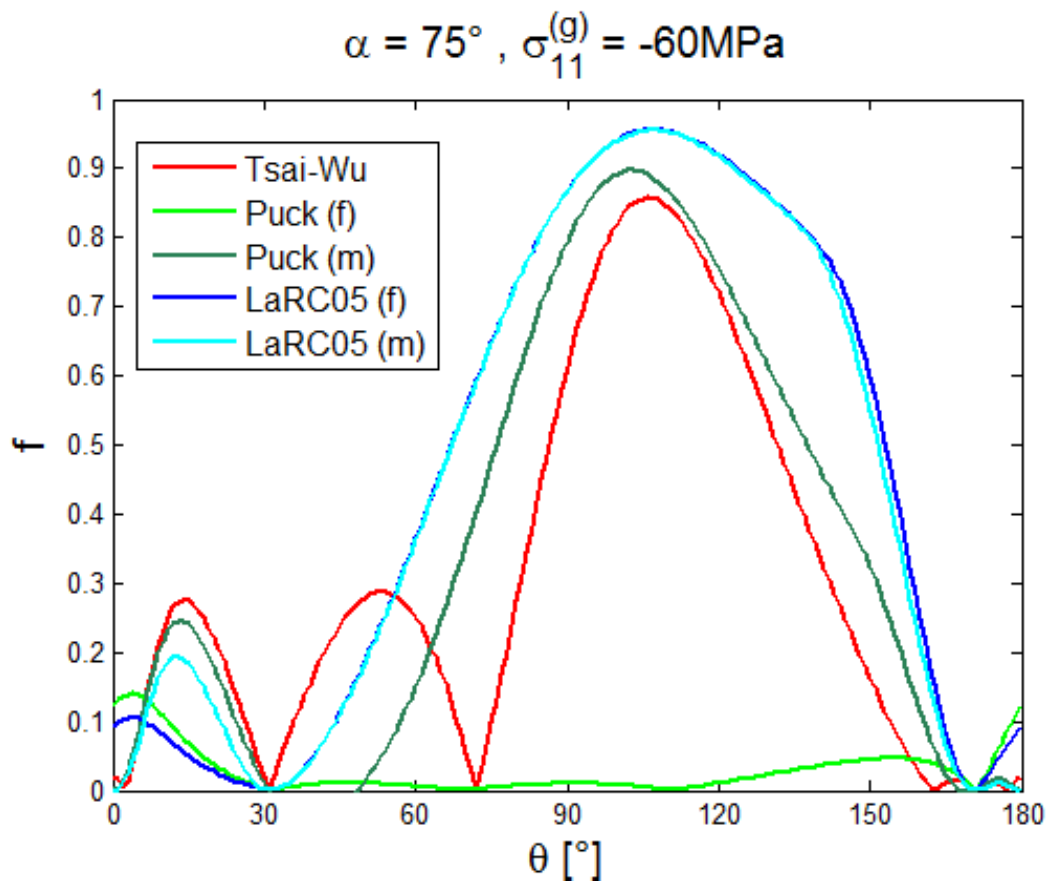

Figura 29 - Variação das funções de falha na borda do furo para o carregamento de compressão uniaxial com $\sigma_{11}^{(g)}=-60 M P a$ e $\alpha=75^{\circ}$ no laminado unidirecional

As distribuições de tensões em coordenadas do material para um carregamento de cisalhamento puro são mostradas na Figura 30. Para materiais isotrópicos, cisalhamento puro é equivalente ao carregamento biaxial de tração/compressão e induz tensões máximas 
e mínimas de $\pm 4 \sigma_{n}$, uma vez que o carregamento trativo resulta em máximos e mínimos de $3 \sigma_{n}$ e $-1 \sigma_{n}$ e o princípio da superposição pode ser diretamente aplicado, enquanto para materiais anisotrópicos, mesmo em regime elástico, é necessário tomar cuidado com a direção das coordenadas do material, pois o mesmo carregamento pode ter efeito diferente se o ângulo entre o mesmo e as fibras, no caso de lâminas unidirecionais, for diferente.

Por exemplo: considerando um carregamento de cisalhamento puro tal que $\sigma_{12}^{(g)}=\sigma_{n}$ e as fibras tenham uma orientação genérica $\alpha$, pode-se utilizar o princípio da superposição para somar o efeito de um carregamento trativo com $\sigma_{11}^{(g)}=\sigma_{n}$ e um carregamento compressivo com $\sigma_{22}^{(g)}=-\sigma_{n}$, ambos considerando a orientação das fibras $\alpha-45^{\circ}$ (ou $\sigma_{11}^{(g)}=-\sigma_{n}$ e $\sigma_{22}^{(g)}=\sigma_{n}$ com orientação das fibras $\left.\alpha+45^{\circ}\right)$. O princípio da superposição é de fundamental importância na modelagem de sistemas mecânicos e pode ser utilizado em quaisquer materiais em regime linear e elástico. A única condição necessária para aplica-lo é que a resposta da estrutura seja relacionada com a solicitação de forma linear, independente de qualquer tipo de anisotropia. Note em particular que o valor de $\sigma_{11}$ mostrado na Figura 30 é muito maior do que o $\mathrm{K}_{\mathrm{t}}$ para materiais isotrópicos.

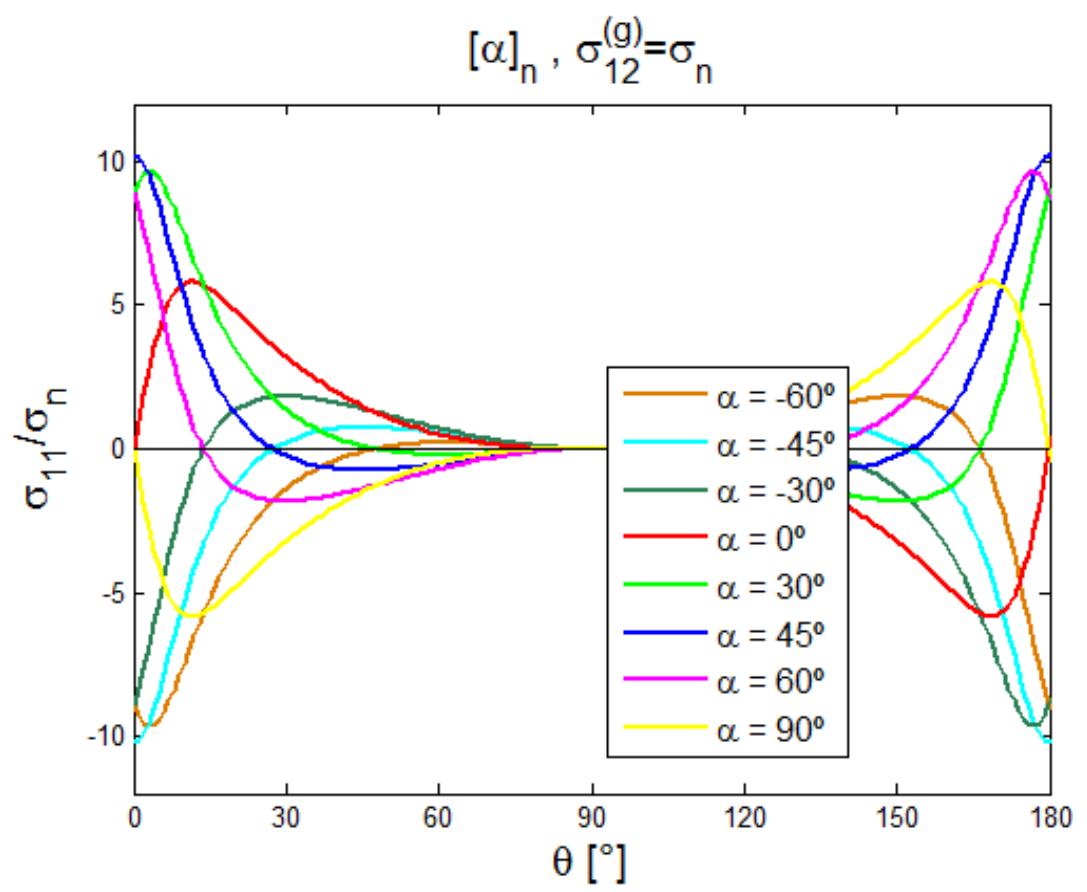

Figura 30 - Tensões ao longo da borda do furo em coordenadas do material para uma placa grande de laminado unidirecional com furo circular e carregada por cisalhamento puro (continua) 

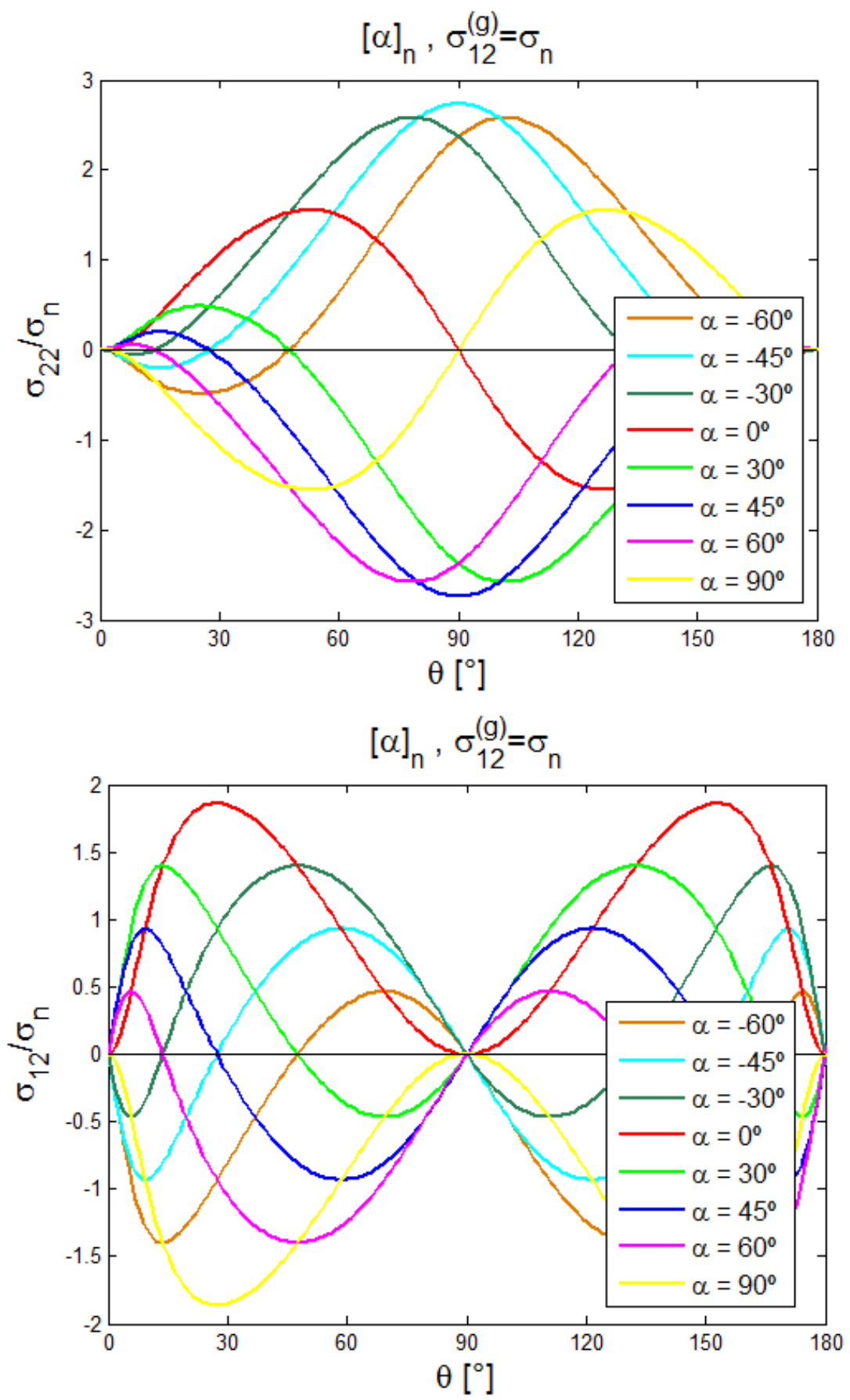

Figura 30 - Tensões ao longo da borda do furo em coordenadas do material para uma placa grande de laminado unidirecional com furo circular e carregada por cisalhamento puro (continuação)

As estimativas de resistências para o carregamento cisalhante são mostradas na Figura 31. Pode-se notar de forma clara duas regiões de comportamento bem distintos: para $40^{\circ}<\alpha<50^{\circ}$, as resistências estimadas são praticamente constantes e aproximadamente 
iguais a $0.78 S_{12}$, onde $S_{12}$ é a resistência ao cisalhamento medida em um corpo de prova sem entalhe; caso contrário, a curva de resistência tem um comportamento suave, sendo mínima para $\alpha=-45^{\circ}$. Apesar da curva de resistência para o carregamento biaxial de tração/compressão não ser mostrada para evitar informações excessivas não necessárias no gráfico, a mesma pode ser utilizada para um entendimento da Figura 31 seguindo o raciocínio desenvolvido anteriormente para o princípio da superposição. A resistência é máxima quando a tensão trativa está atuando paralelamente às fibras e o compressivo está atuando na matriz ( $\alpha=45^{\circ}$ para cisalhamento puro e $\alpha=0^{\circ}$ para tração/compressão) e mínima quando a tração está atuando na matriz e a compressão nas fibras $\left(\alpha=-45^{\circ}\right.$ para cisalhamento puro e $\alpha= \pm 90^{\circ}$ para tração/compressão) porque a matriz tem comportamento frágil, logo, tem resistência menor à tração, ao contrario da fibra que suporta melhor o carregamento trativo.

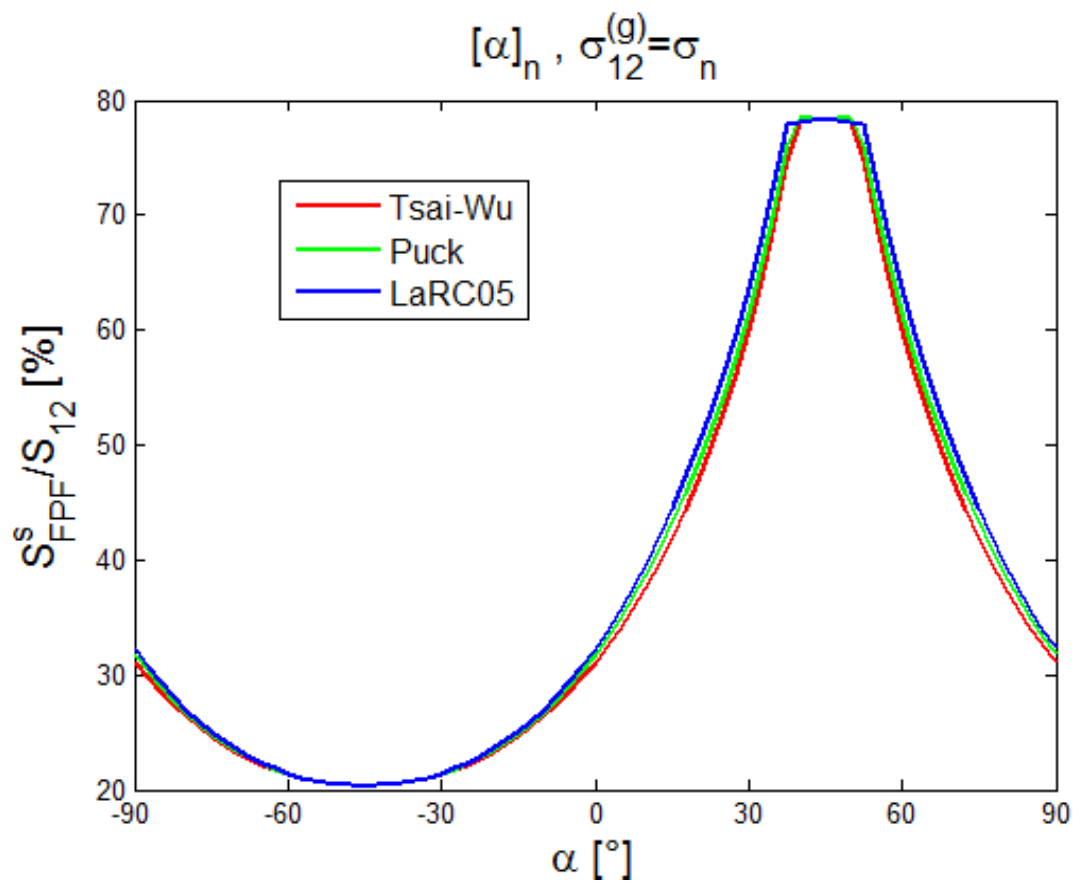

Figura 31 - Estimativas de $S_{F P F}^{s} / S_{12}$ para placas grandes de laminados unidirecionais com furos circulares

As resistências normalizadas para os carregamentos de tração e compressão têm valores menores que $8 \%$, tendo em vista que as mesmas são comparadas com resistências medidas em corpos de prova não entalhados. No entanto, as resistências utilizadas para a 
normalização são as medidas paralelamente às fibras, que é a direção em que a lâmina é mais resistente. Por outro lado, para o caso do cisalhamento puro aplicado, a falha de placas grandes com furos circulares alcançam valores entre $20 \%$ e $80 \%$ da resistência ao cisalhamento da lâmina. Este fato se dá porque, quando há entalhe, a falha da matriz tende a ser o mecanismo dominante na maioria dos casos, como por exemplo, quando se aplica cisalhamento. Dessa forma, esta é a razão pela qual a resistência normalizada ao cisalhamento não é tão sensível ao entalhe, ao contrário do que acontece com a resistência à tração e à compressão para placas com furos circulares, que são normalizadas utilizando as resistências das fibras. Para exemplificar o raciocínio desenvolvido, a Figura 32 mostra as resistências normalizadas em relação às propriedades medidas perpendicularmente às fibras. Para tração, a razão $S_{F P F}^{t} / S_{22}^{t}$ pode chegar a valores maiores do que $200 \%$. Todavia, a comparação com as maiores resistências é mais importante, como feito na Figura 26, porque as propriedades mecânicas da matriz não são suficientes para a maioria das aplicações. Se as resistências e a rigidez da matriz já fossem úteis não haveria a necessidade de reforçá-las com fibras.

Duas condições de carregamento diferentes foram selecionadas para um estudo mais detalhado dos critérios de falha aplicados ao carregamento de cisalhamento puro: $\sigma_{12}^{(g)}=42.5 \mathrm{MPa}$ e $\alpha=30^{\circ}$; e $\sigma_{12}^{(g)}=50 \mathrm{MPa}$ e $\alpha=45^{\circ}$. A Figura 33 mostra as estimativas das funções de falha para um carregamento cisalhante de tensão nominal igual a $42.5 \mathrm{MPa}$ e inclinação da fibra $\alpha=30^{\circ}$. Apesar das diferentes estimativas ao longo da borda do furo, o ponto crítico indicado por todos os critérios é similar, i.e. $\theta \cong 20^{\circ}$ (menos para a fibra sob compressão de acordo com LaRC05, mas o mesmo critério indica falha da matriz anterior à falha da fibra). Esse gráfico também ilustra a tendência que pode ser estendida para todos os outros gráficos de que para a matriz sob tração, Tsai-Wu tende a indicar o maior dano, enquanto para a matriz sob compressão, Tsai-Wu normalmente indica o menor dano. Nessa conclusão, "dano" refere-se ao valor da função que descreve a falha, podendo a falha ocorrer ou não. 

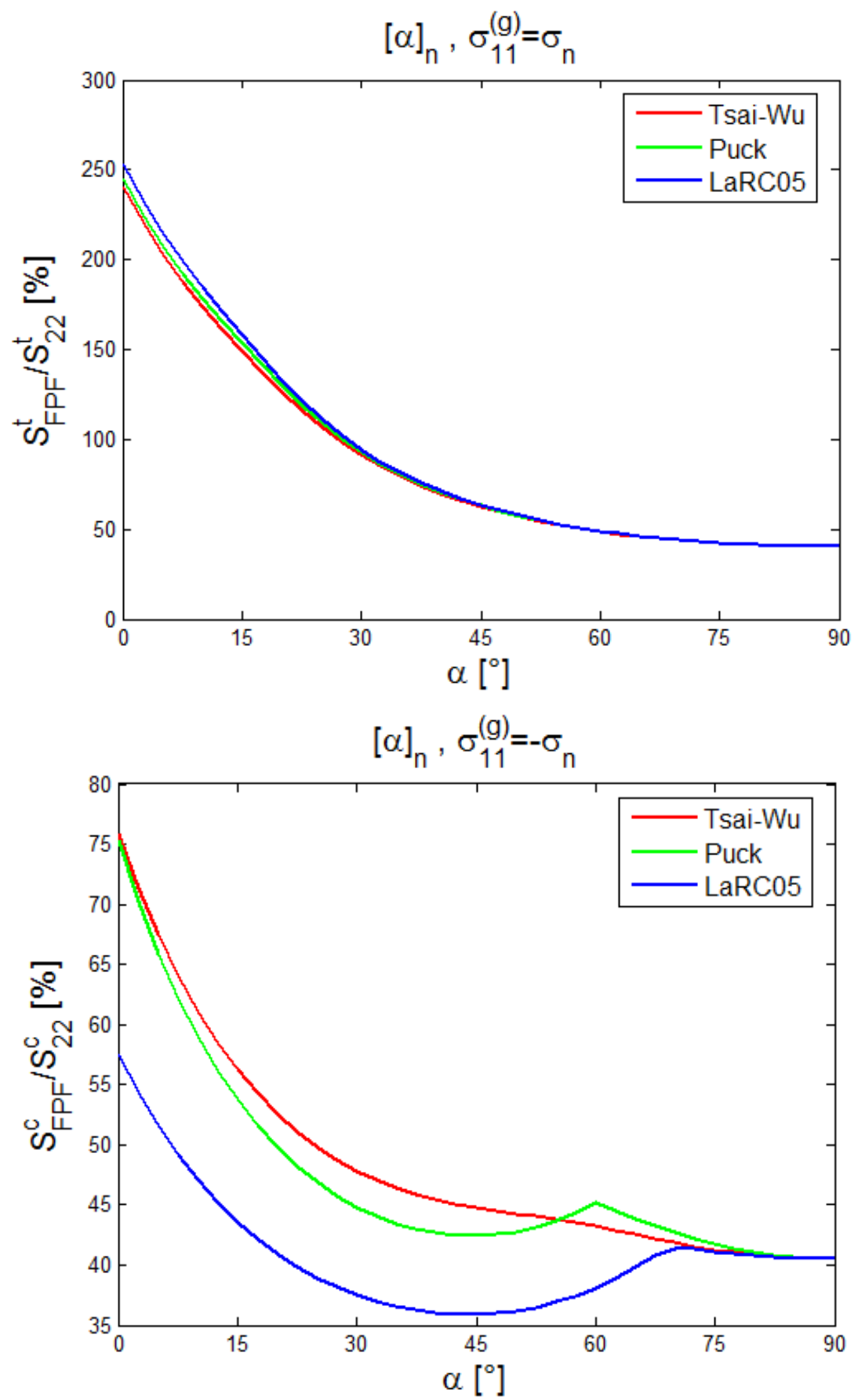

Figura 32 - Estimativas de $S_{F P F}^{t} / S_{22}^{t}$ e $S_{F P F}^{c} / S_{22}^{c}$ para placas grandes de laminados unidirecionais com furos circulares 


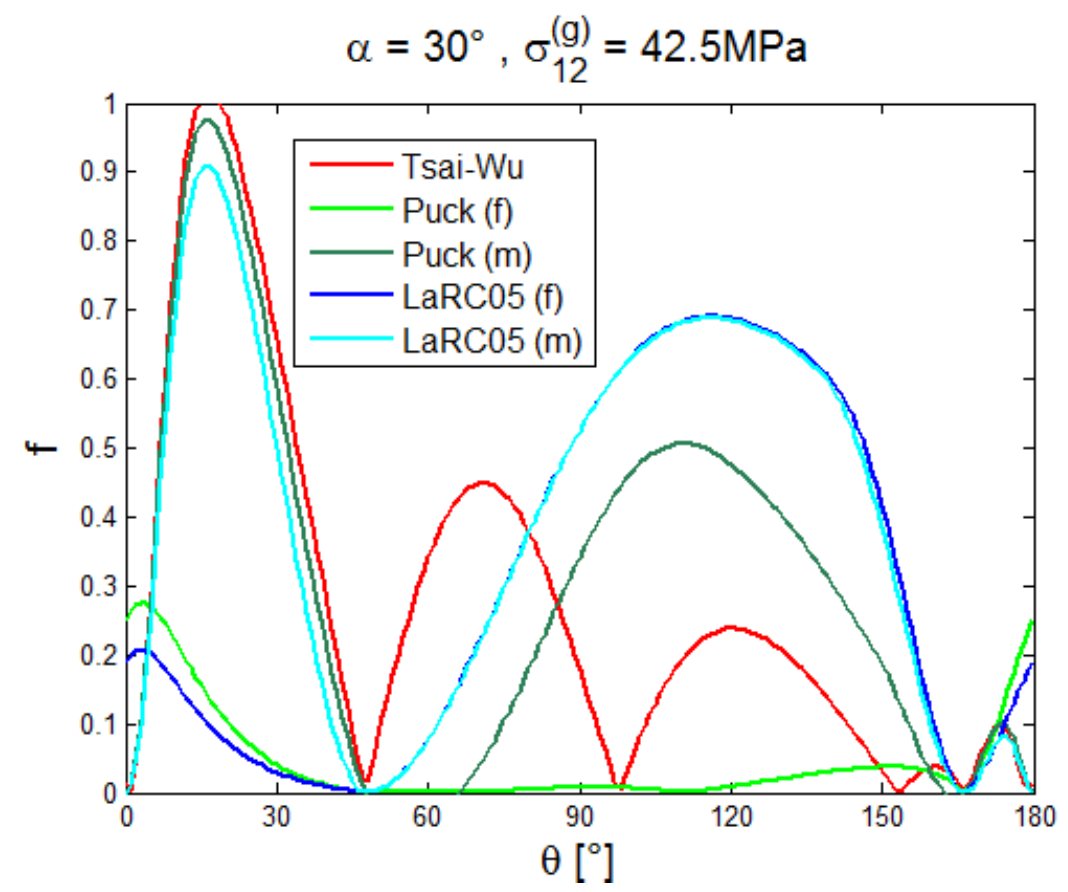

Figura 33 - Variação das funções de falha na borda do furo para o carregamento de cisalhamento puro com $\sigma_{12}^{(g)}=42.5 M P a$ e $\alpha=30^{\circ}$ no laminado unidirecional

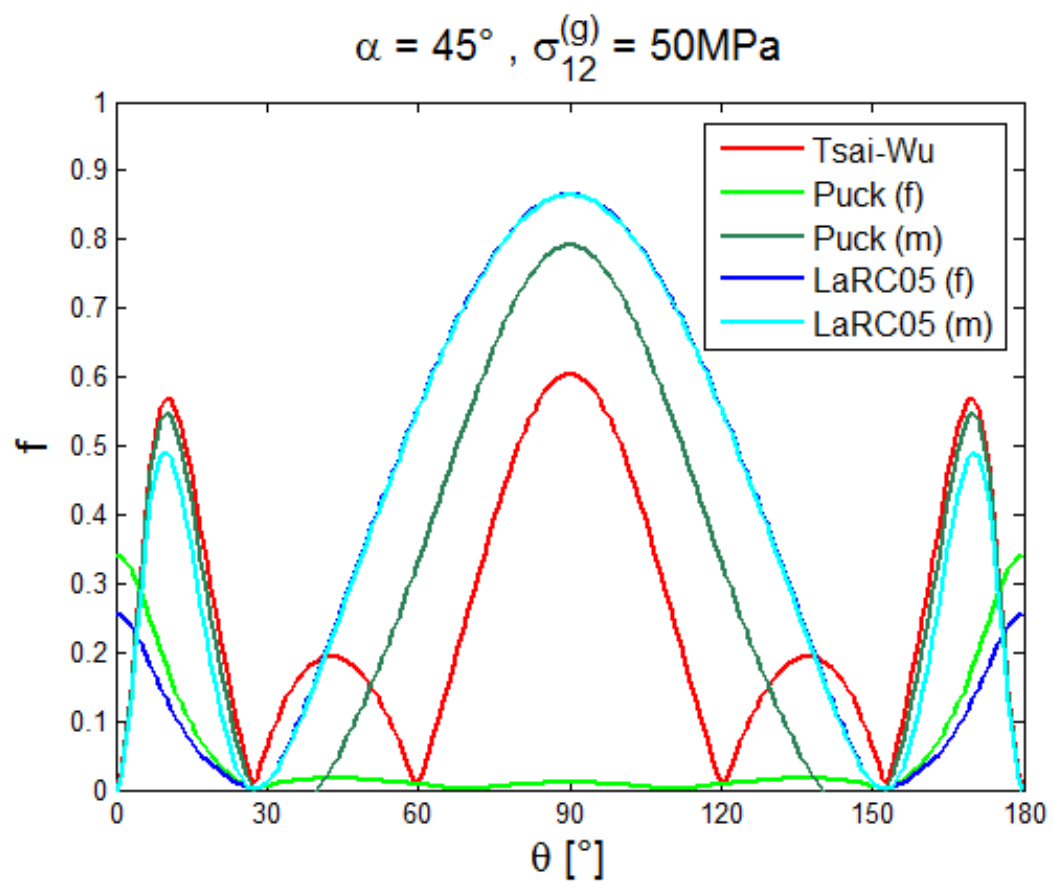

Figura 34 - Variação das funções de falha na borda do furo para o carregamento de cisalhamento puro com $\sigma_{12}^{(g)}=50 M P a$ e $\alpha=45^{\circ}$ no laminado unidirecional 
A Figura 34 mostra a variação dos modelos de falha para um carregamento de cisalhamento puro de 50MPa e inclinação das fibras de $\alpha=45^{\circ}$. Para essa tensão nominal, nenhum critério indica falha, no entanto, suas tendências podem ser analisadas. Novamente, pode-se perceber a uma grande diferença entre as estimativas de dano da fibra de acordo com Puck e com LaRC05; enquanto Puck estima $f<0.1$ para $\theta=90^{\circ}$, LaRC05 resulta em $f \cong 0.9$, tornando mais uma vez evidente a necessidade da validação das hipóteses assumidas por cada modelo durante sua formulação para verificar qual obtém melhor resultado na estimativa de falha das fibras sob compressão.

Uma vez que os três possíveis tipos de carregamentos simples foram estudados torna-se útil avaliar o efeito de carregamentos multiaxiais. Para isso, dois diferentes carregamentos biaxiais foram estudados: tração/tração (t-t) e compressão/compressão (c-c). Ambos os componentes de tensões são independentes do valor de $\alpha$, como mostrado na Figura 35. Esse resultado se torna evidente pelo círculo de Mohr; nesse plano, o círculo se torna um ponto ou, com outras palavras, um círculo de raio igual a zero, e nenhuma diferença é percebida quando há uma rotação do elemento diferencial no plano. Dessa forma, a inclinação das fibras não deve influenciar o resultado.

Todos os modelos resultaram em estimativas de resistência exatamente iguais para ambos os carregamentos: $S_{F P F}^{t-t}=17.7 M P a$ e $S_{F P F}^{c-c}=-68.1 M P a$. Note que esses dois valores podem ser reescritos como: $S_{F P F}^{t-t}=2.2 S_{22}^{t}$ e $S_{F P F}^{c-c}=2.2 S_{22}^{c}$, o que indica que a matriz falha por tração ou compressão pura, justificando então as estimativas iguais. Todavia, para comprovar essa afirmação, a função das funções de falha ao longo da borda do furo devem ser analisadas.

As Figuras 36 e 37 mostram que, apesar das funções serem diferentes antes da falha (principalmente para c-c), isto é, $\sigma_{n}=15 M P a$ para t-t e $\sigma_{n}=-60 M P a$ para c-c, quando a tensão nominal se aproxima da resistência estimada os modelos se tornam equivalentes, porque para $\theta=90^{\circ}$, que é onde acontece a falha, a matriz está sob carregamento axial puro (ver a variação de $\sigma_{22}$ de acordo $\operatorname{com} \theta$ na Figura 35). Outro ponto interessante é que apesar das estimativas de resistências para carregamento estática serem iguais para esses carregamentos biaxiais, estimativas de resistência para carregamentos cíclicos tendem a ser diferentes. 

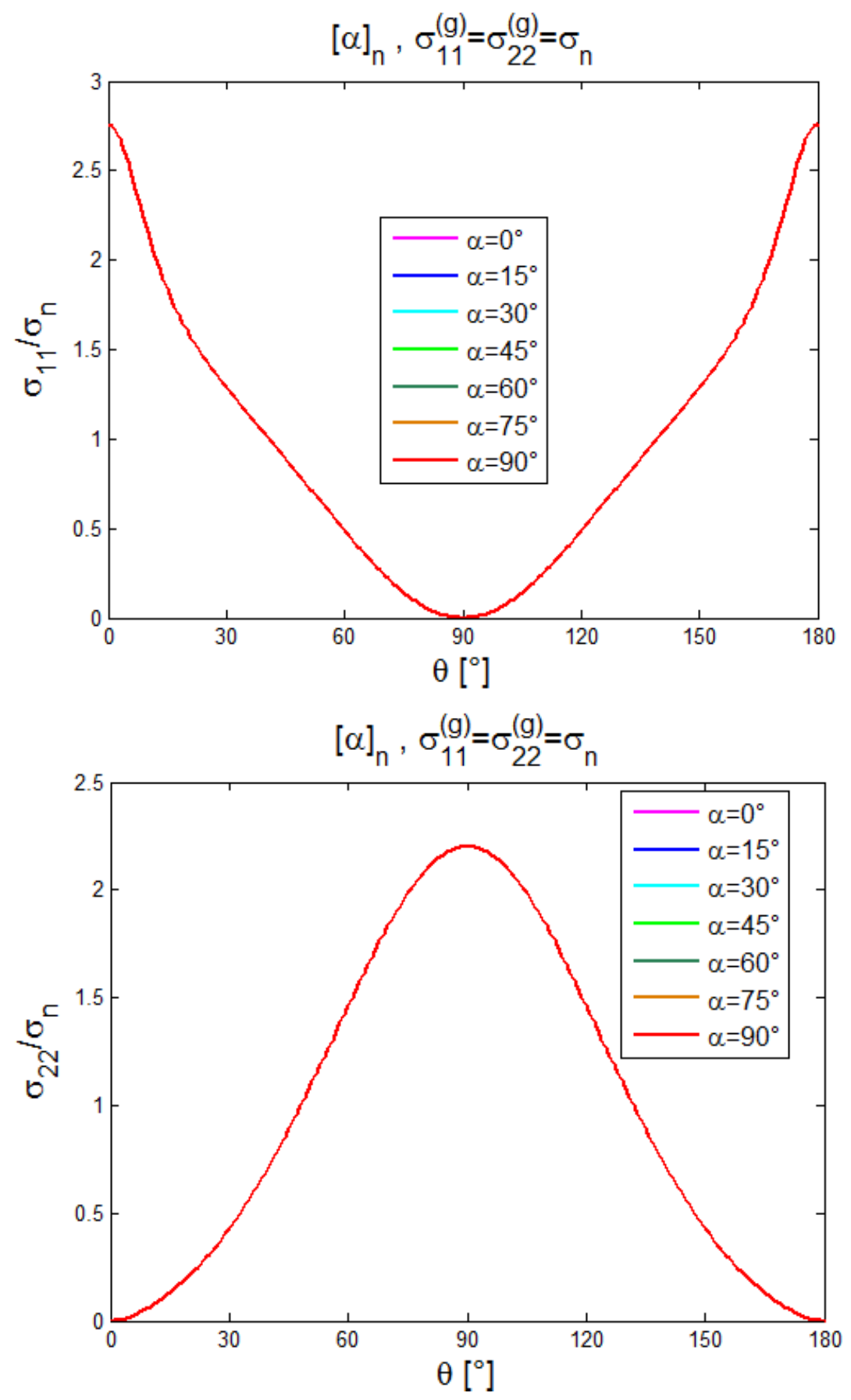

Figura 35 - Tensões ao longo da borda do furo em coordenadas do material para uma placa grande de laminado unidirecional com furo circular e carregada biaxialmente (continua) 


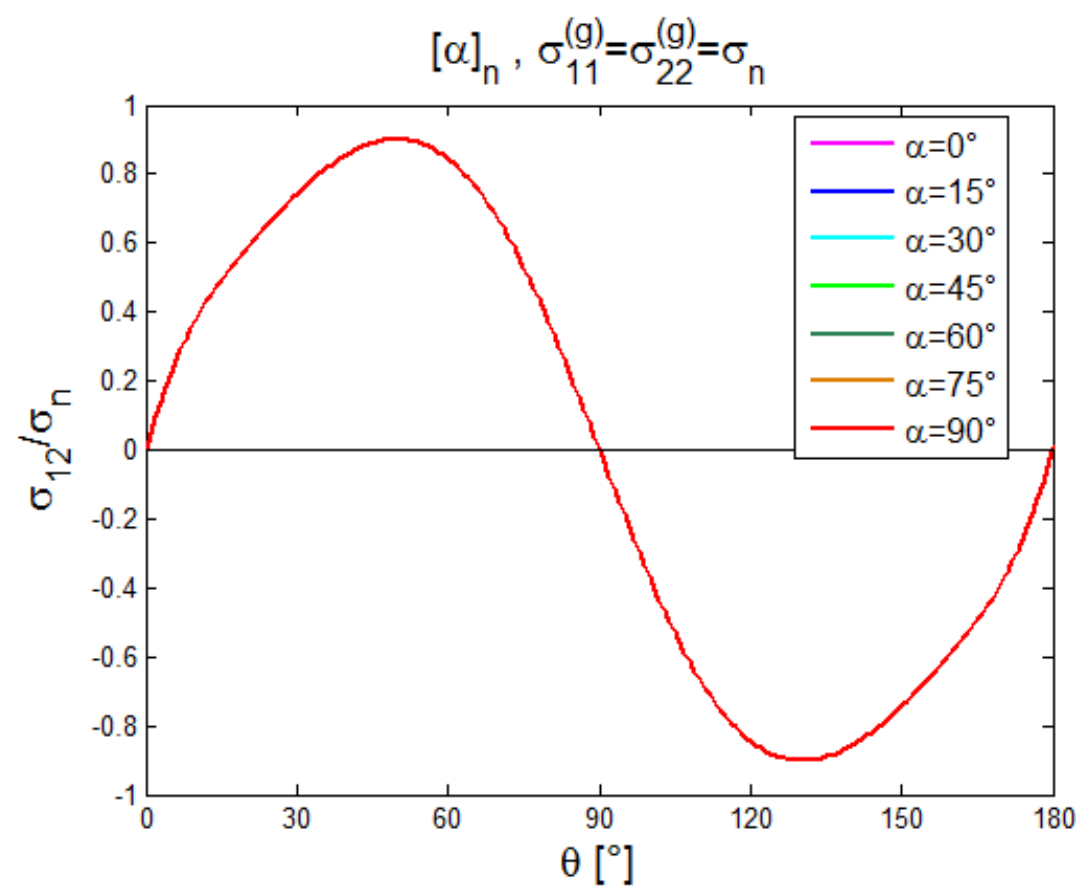

Figura 35 - Tensões ao longo da borda do furo em coordenadas do material para uma placa grande de laminado unidirecional com furo circular e carregada biaxialmente (continuação)

Tendo em vista que, para ligas metálicas, o material pode falhar por fadiga mesmo sem haver escoamento do ponto de vista macroscópico, espera-se que os laminados falhem por fadiga para valores da função de falha estática menor do que um, sendo então a forma da função para valores menores do que um também importante. Todavia, esse tópico não faz parte do escopo do presente estudo e não será aprofundado, mas vale lembrar que concentradores de tensões influenciam principalmente a vida da fadiga.

Como resultado genérico das Figuras 25-37, estima-se que a matriz falhe antes das fibras, exceto para alguns casos da fibra sob compressão pelo modelo LaRC05. Todos os modelos tiveram resultados que indicam que para tração uniaxial e para cisalhamento puro, as direções das fibras $\alpha=0^{\circ}$ e $\alpha=45^{\circ}$, respectivamente, apesar de resultarem em uma maior concentração de tensão, são condições ótimas em relação às resistências. Dessa forma, fica claro que minimizar a concentração de tensão não é necessariamente uma boa metodologia de projeto, sendo desejável em alguns casos até mesmo maximizar a concentração de tensão para aumentar a resistência. A afirmação anterior deve ser interpretada com cuidado; concentradores de tensão não são desejáveis do ponto de vista de integridade estrutural e devem ser evitados, todavia em alguns casos, como discutido 
anteriormente, se o concentrador de tensão for inevitável, a posição das fibras que maximizem a tensão ao redor do entalhe pode apresentar a condição em que as funções de falha sejam minimizadas.
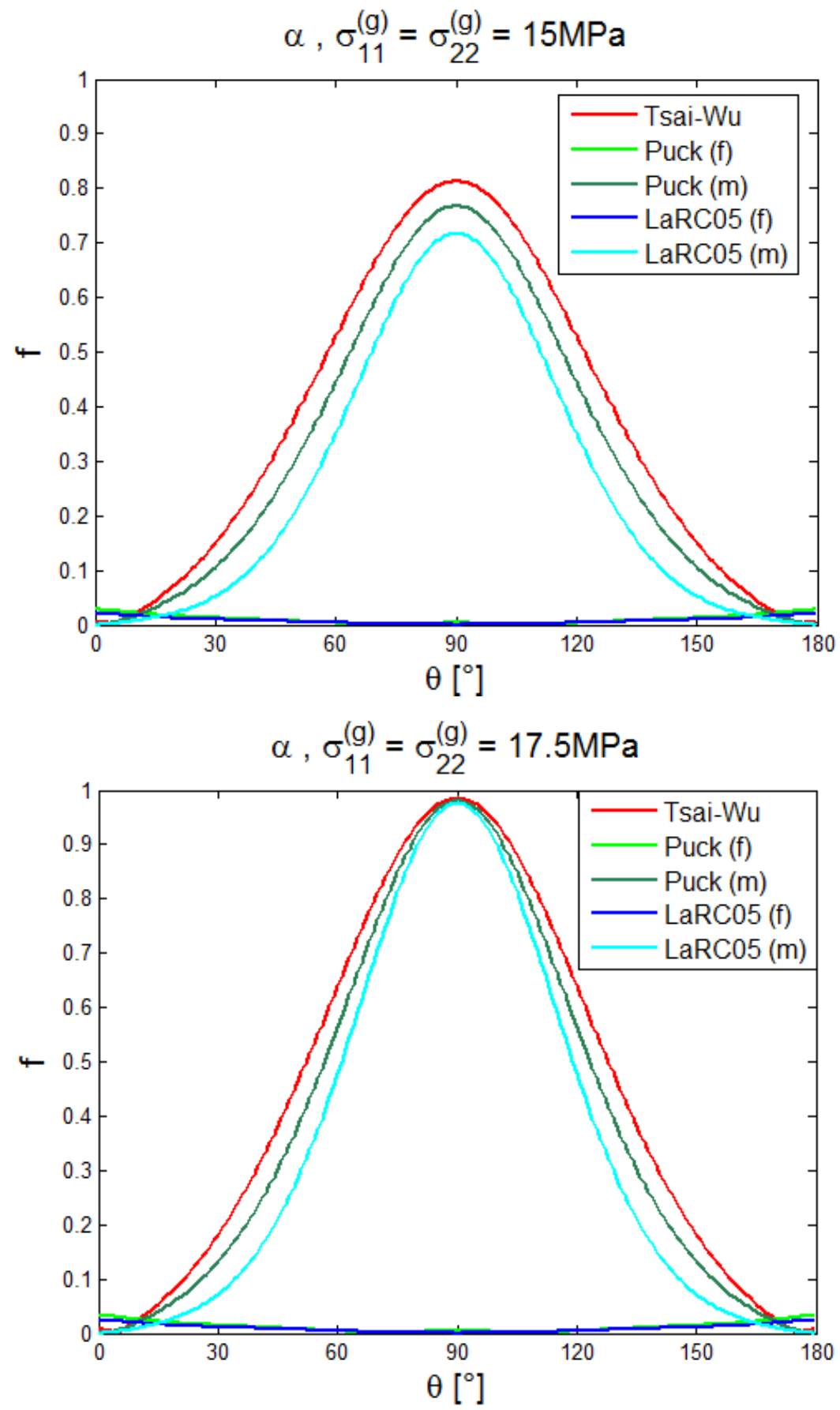

Figura 36 - Variação das funções de falha na borda do furo para o carregamento de biaxial com $\sigma_{11}^{(g)}=\sigma_{22}^{(g)}=15 \mathrm{MPa}$ e $\sigma_{11}^{(g)}=\sigma_{22}^{(g)}=17.5 \mathrm{MPa}$ para qualquer valor de $\alpha$ no laminado unidirecional 

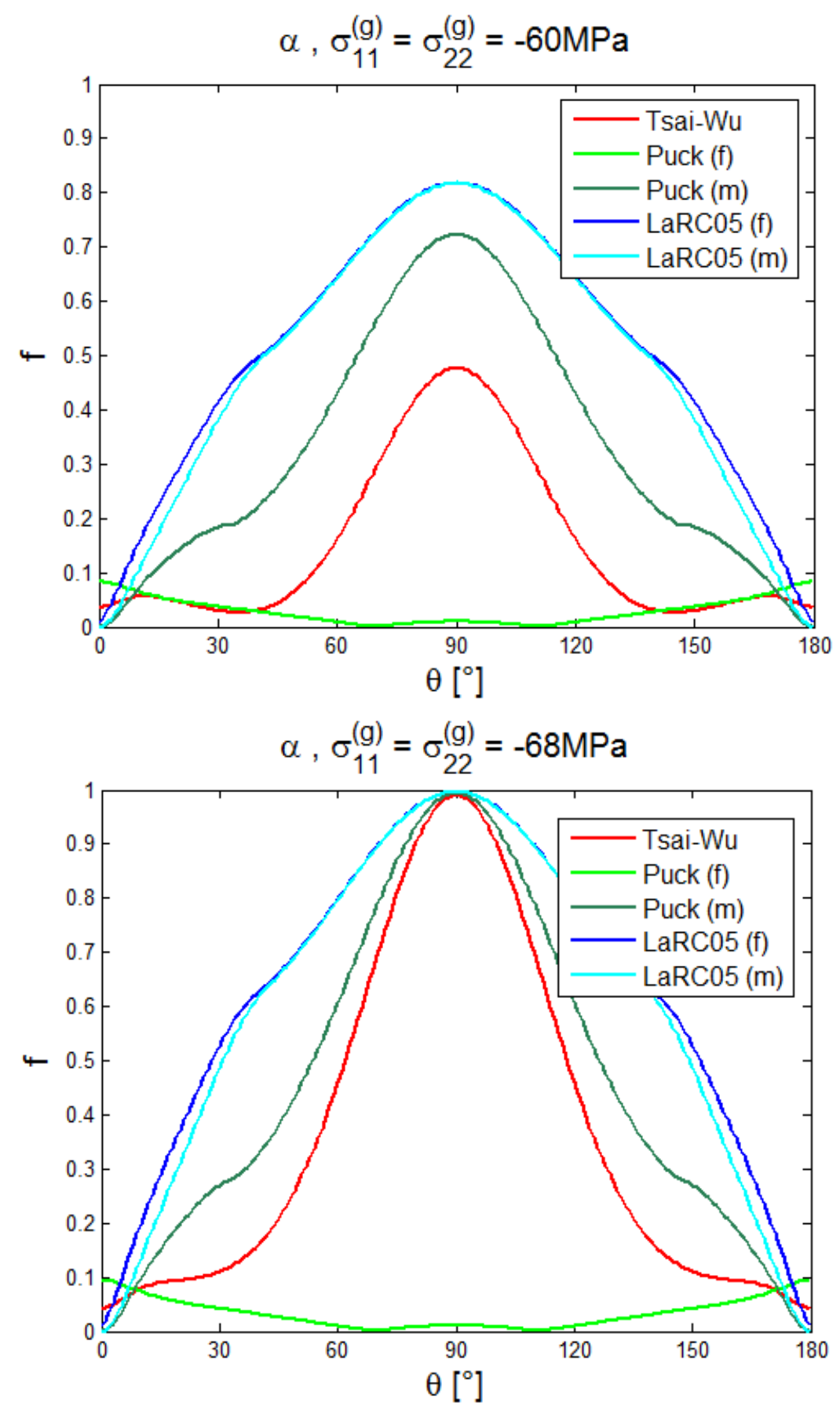

Figura 37 - Variação das funções de falha na borda do furo para o carregamento de biaxial com $\sigma_{11}^{(g)}=\sigma_{22}^{(g)}=-60 M P a$ e $\sigma_{11}^{(g)}=\sigma_{22}^{(g)}=-68 M P a$ para qualquer valor de $\alpha$ no laminado unidirecional

Vale também ressaltar que, embora o modelo LaRC05 possa subestimar a resistência das fibras sob compressão porque não necessariamente o desalinhamento da 
mesma coincide com a região de concentração de tensão, esse desalinhamento é um valor pequeno, normalmente menor do que cinco graus. A principal diferença entre as estimativas para falha da fibra sob compressão entre Puck e LaRC05 se deve às diferentes hipóteses de falha assumidas. LaRC05 modela a falha da fibra sob compressão considerando sua instabilidade.

Outro ponto importantíssimo mostrado é que enquanto o valor nominal da tensão que pode ser aplicada sem resultar em escoamento de uma placa grande de material isotrópico diminui 3 vezes quando um furo circular é incluído em uma placa grande sob tração ou compressão e 4 vezes quando se tem uma condição de cisalhamento puro, para o laminado estudado a resistência (ao início do dano) diminui aproximadamente 20 vezes para tração, 12,5 para compressão e 1,25 para cisalhamento. Mostrando, então, claramente os possíveis perigos em problemas de concentrações de tensões em materiais anisotrópicos.

\subsection{Estimativas de resistências para laminados $[ \pm \alpha]_{n s}$ com furo circular}

Na prática, o uso de laminados unidirecionais $\left([\alpha]_{n}\right)$ é limitado por aumentar a resistência e a rigidez da estrutura em apenas uma direção e carregamentos reais são normalmente multiaxiais. Por outro lado, o entendimento do laminado $[\alpha]_{n}$ é justificado pelo entendimento fenomenológico da modelagem. Ao mesmo tempo, fazer um estudo sobre materiais compósitos envolvendo todas as possíveis variáveis não se torna viável, como comentado por Tsai e Melo (2014). Por conta disso, os laminados do tipo $[ \pm \alpha]_{n s}$ (angle-ply) serão estudados a seguir.

Na Figura 21 foram mostradas as estimativas de resistência à tração e à compressão para laminados $[\alpha]_{n}$ e $[ \pm \alpha]_{n s}$ sem entalhes utilizando os 3 critérios de falha estudados. $\mathrm{O}$ objetivo dessa seção é estender essa análise para placas grandes com furos circulares. Primeiro, usando a TCL, é possível concluir que para $[ \pm \alpha]_{n s}$ não existe o acoplamento entre tensões e deformações normais e cisalhantes porque os elementos nas matrizes de rigidez e de flexibilidade equivalentes responsáveis por esse efeito são nulos, ao contrário do que acontece para os laminados $[\alpha]_{n}$ se $\alpha \neq 0^{\circ}, \pm 90^{\circ}$. Outro ponto importante é que $[+\alpha]_{n}$ e $[-\alpha]_{n}$ são dois laminados diferentes, enquanto $[ \pm \alpha]_{n s}$ é um laminado que contém lâminas nas 
quais as fibras têm orientação $+\alpha$ e $-\alpha$. Então, para carregamentos não simétricos, como por exemplo o caso do cisalhamento puro, as lâminas $+\alpha$ e $-\alpha$ não têm o mesmo efeito, como discutido anteriormente, e a falha (ou pelo menos a falha inicial que é o objetivo de estudo do presente trabalho) será dada pela lâmina mais crítica. Para carregamentos uniaxiais ambas têm o mesmo efeito e a distribuição de tensão é mostrada na Figura 38. Note que apesar das distribuiçõoes de tensões parecerem similares às mostradas na Figura 25, uma diferença significativa é notada em alguns casos como para $\alpha=30^{\circ}$.

As estimativas de resistências à tração e à compressão para os laminados $[ \pm \alpha]_{n s}$ são mostradas na Figura 39. As mesmas estão sobrepostas às resistências para os laminados unidirecionais para obter uma comparação direta. Claramente $[0]_{n}$ e $[90]_{n}$ devem ser equivalente à $[ \pm 0]_{n s}$ e $[ \pm 90]_{n s}$, respectivamente, mas a variação da resistência de acordo com a inclinação das fibras em relação ao carregamento resulta em estimativas bem diferentes. Para tração e compressão, as curvas de resistência são menos suaves para $[ \pm \alpha]_{n s}$, ao contrário do que acontece para laminados não entalhados como mostrado na Figura 21.

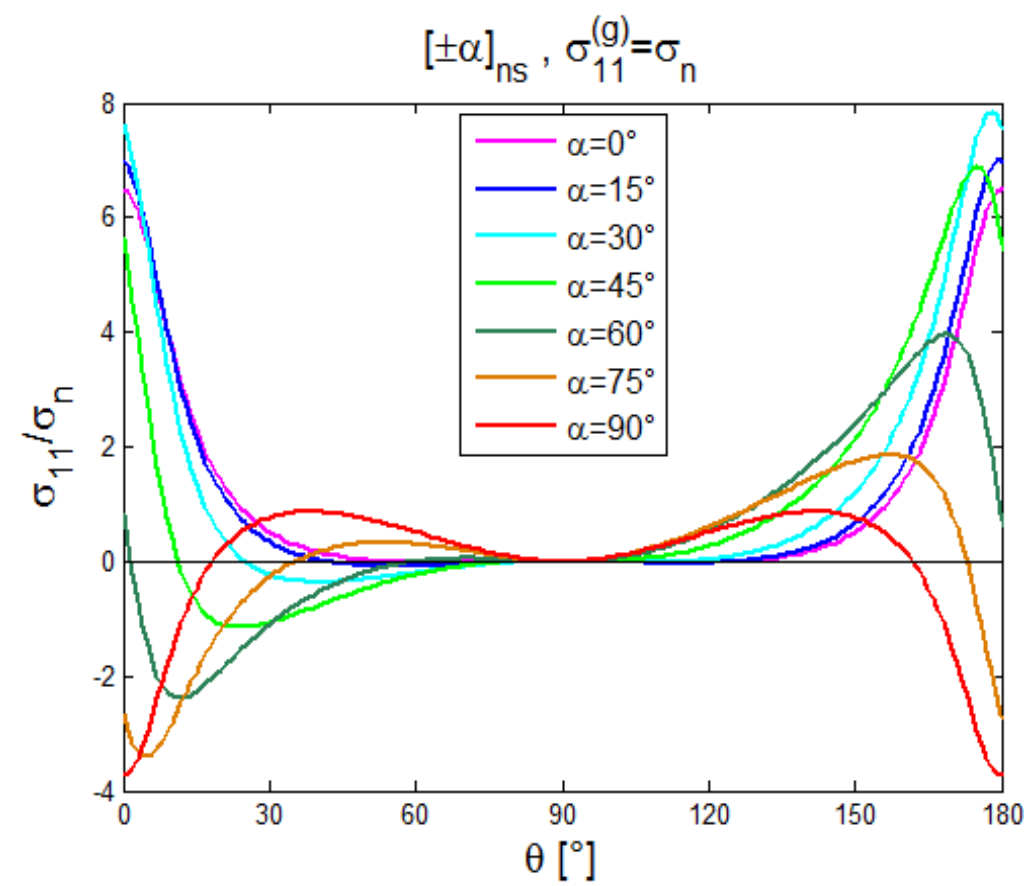

Figura 38 - Tensões ao longo da borda do furo em coordenadas do material para uma placa grande de laminado angle-ply com furo circular e carregada uniaxialmente (continua) 

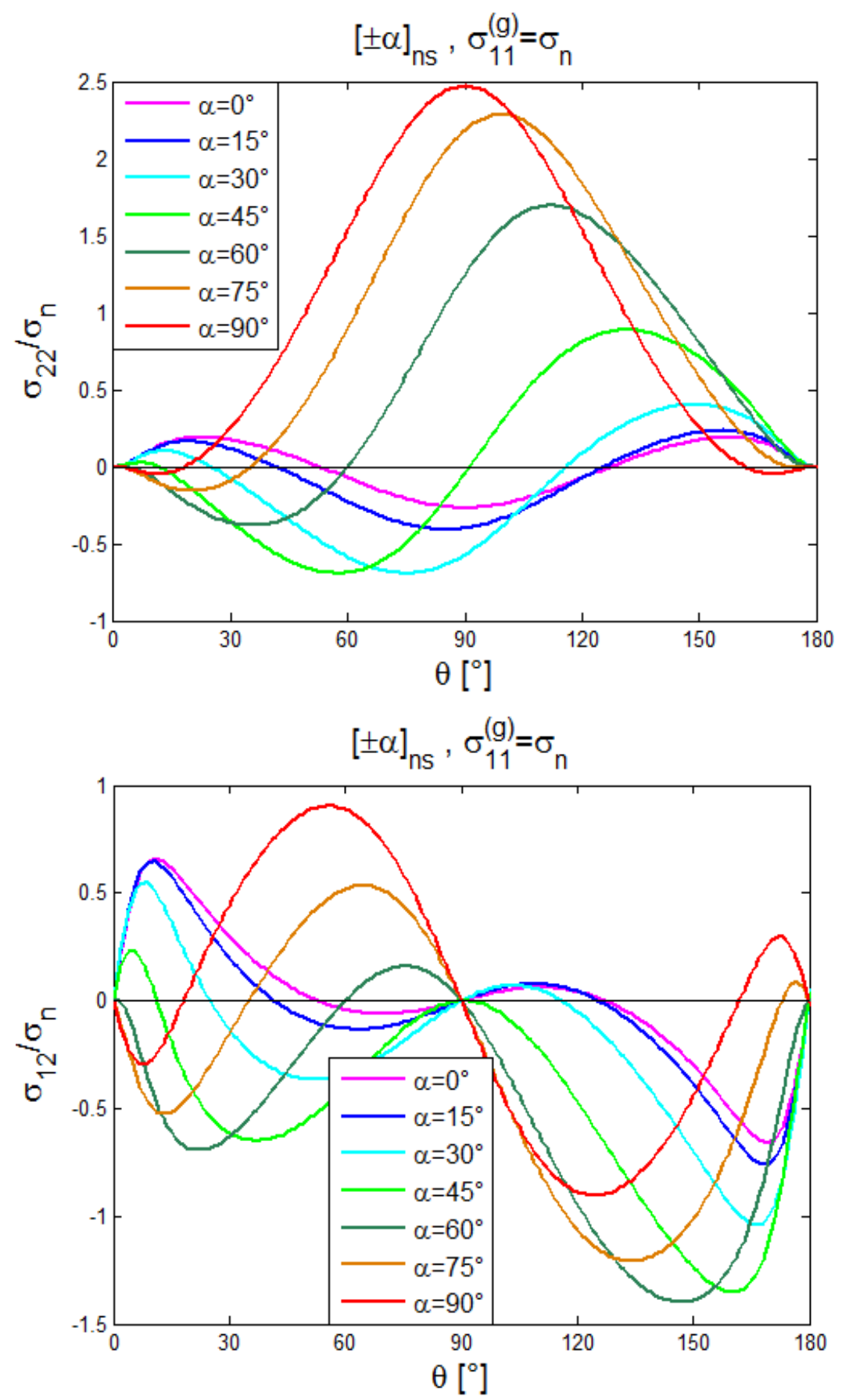

Figura 38 - Tensões ao longo da borda do furo em coordenadas do material para uma placa grande de laminado angle-ply com furo circular e carregada uniaxialmente (continuação) 

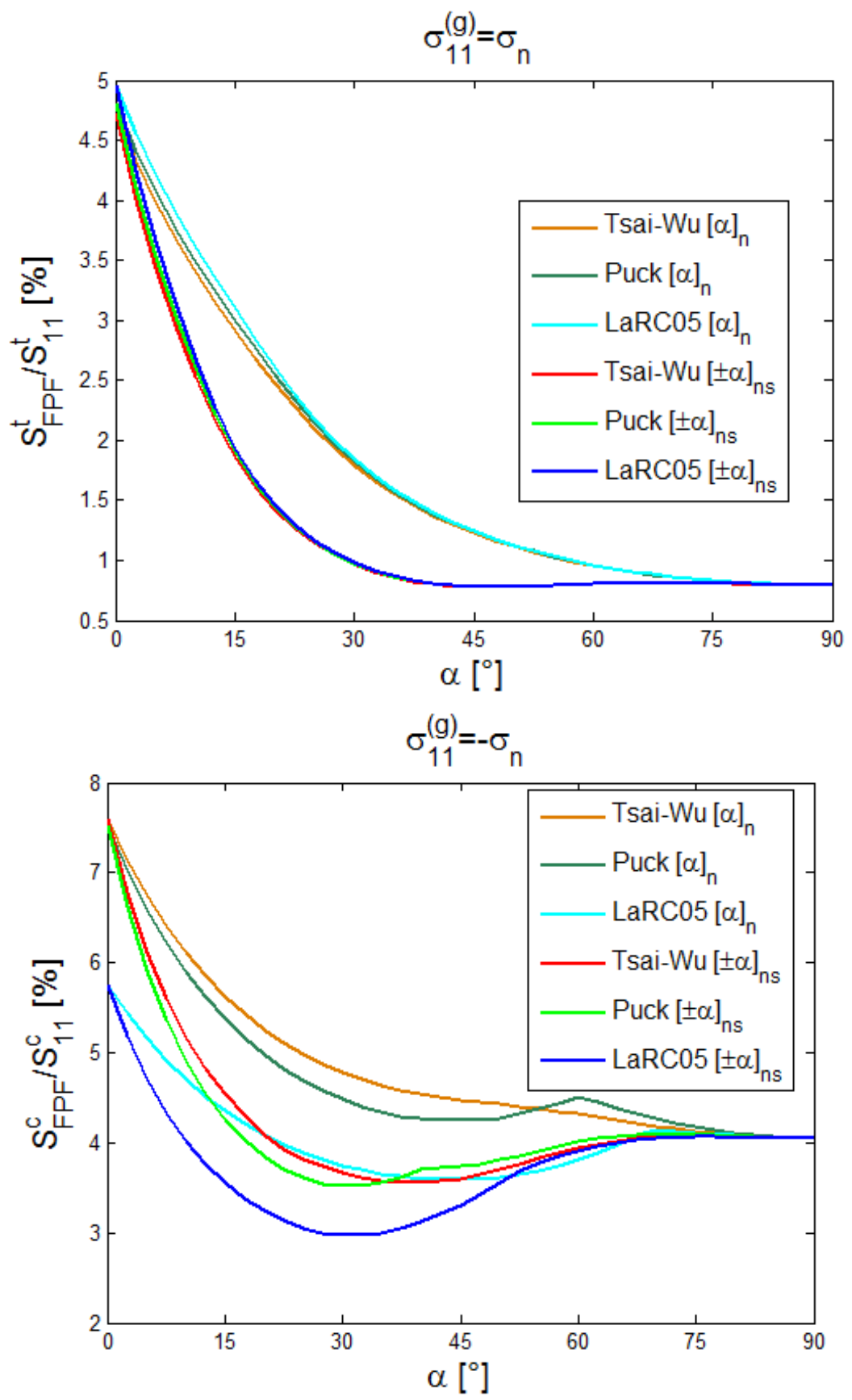

Figura 39 - Estimativas de $S_{F P F}^{t} / S_{11}^{t}$ e $S_{F P F}^{c} / S_{11}^{c}$ para placas grandes de laminados angleply com furos circulares

A Figura 40 mostra como variam as funções de falha ao longo da borda do furo para as mesmas condições de carregamento estudadas em detalhes para $[\alpha]_{n}$ nas Figuras 27-29 (nomeadamente $\sigma_{11}^{(g)}=90 M P a$ e $\alpha=0^{\circ} ; \sigma_{11}^{(g)}=-70 M P a$ e $\alpha=15^{\circ} ;$ e $\sigma_{11}^{(g)}=-60 M P a$ e $\alpha=75^{\circ}$ ) para facilitar as comparações. 


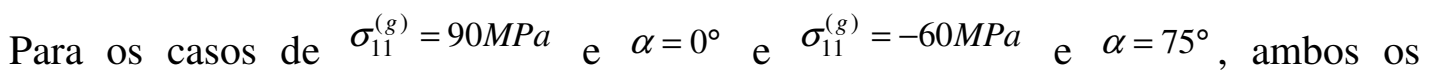
laminados resultam em distribuições parecidas de funções, mas para $\sigma_{11}^{(g)}=-70 M P a$ e $\alpha=15^{\circ}$ todos os modelos indicam falha para o laminado angle-ply, enquanto apenas LaRC05 estima a falha para o laminado unidirecional. Dessa forma, fica exemplificado, então, que, pelo menos para projetos em que seja necessário garantir que não ocorrerá inicio do dano, utilizar laminados angle-ply ao invés de unidirecionais não é necessariamente a melhor opção do ponto de vista da resistência.

As distribuiç̧ões de tensões para uma placa grande de um laminado do tipo angleply submetida a uma tensão de cisalhamento puro são mostradas na Figura 41. Por esse gráfico e os apresentados na Figura 38 pode-se concluir que esse tipo de laminado resulta em concentrações de tensões maiores do que os laminados unidirecionais para os casos estudados.

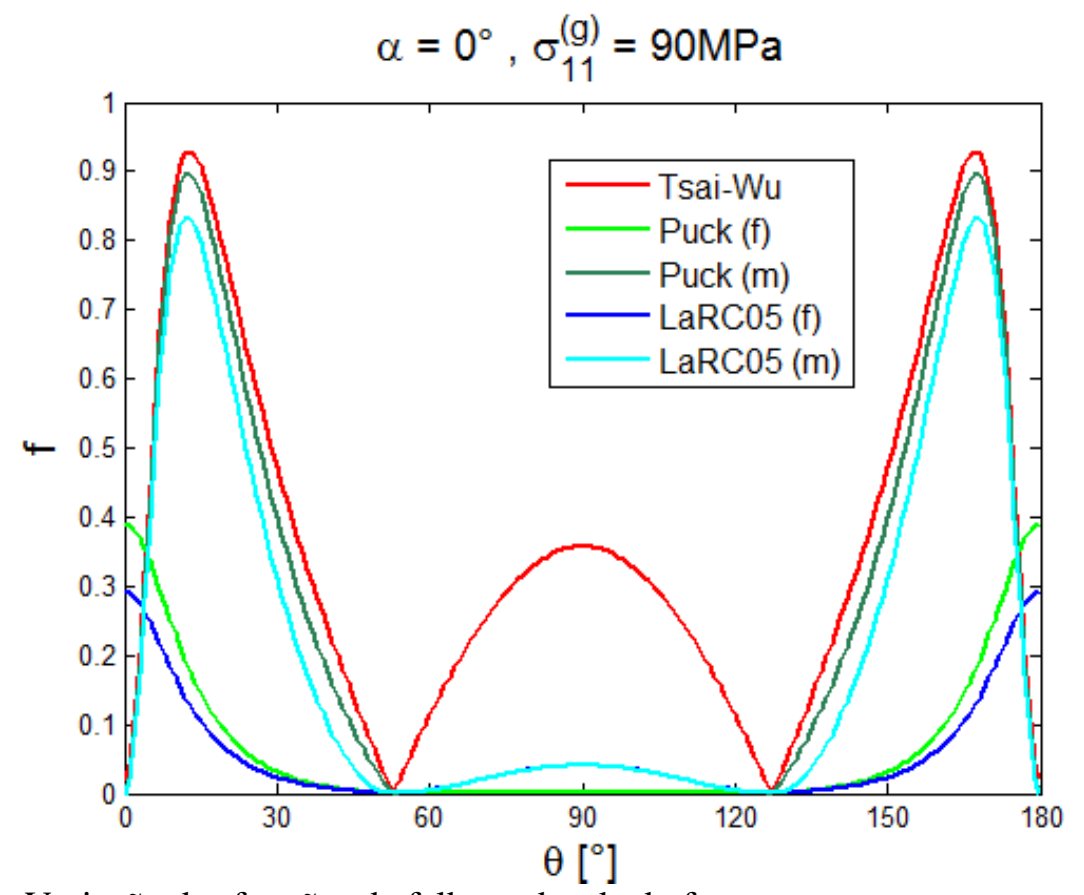

Figura 40 - Variação das funções de falha na borda do furo para carregamentos uniaxiais no laminado angle-ply com: $\sigma_{11}^{(g)}=90 M P a$ e $\alpha=0^{\circ} ; \sigma_{11}^{(g)}=-70 M P a$ e $\alpha=15^{\circ} ;$ e $\sigma_{11}^{(g)}=-60 M P a$ e $\alpha=75^{\circ}$ (continua) 

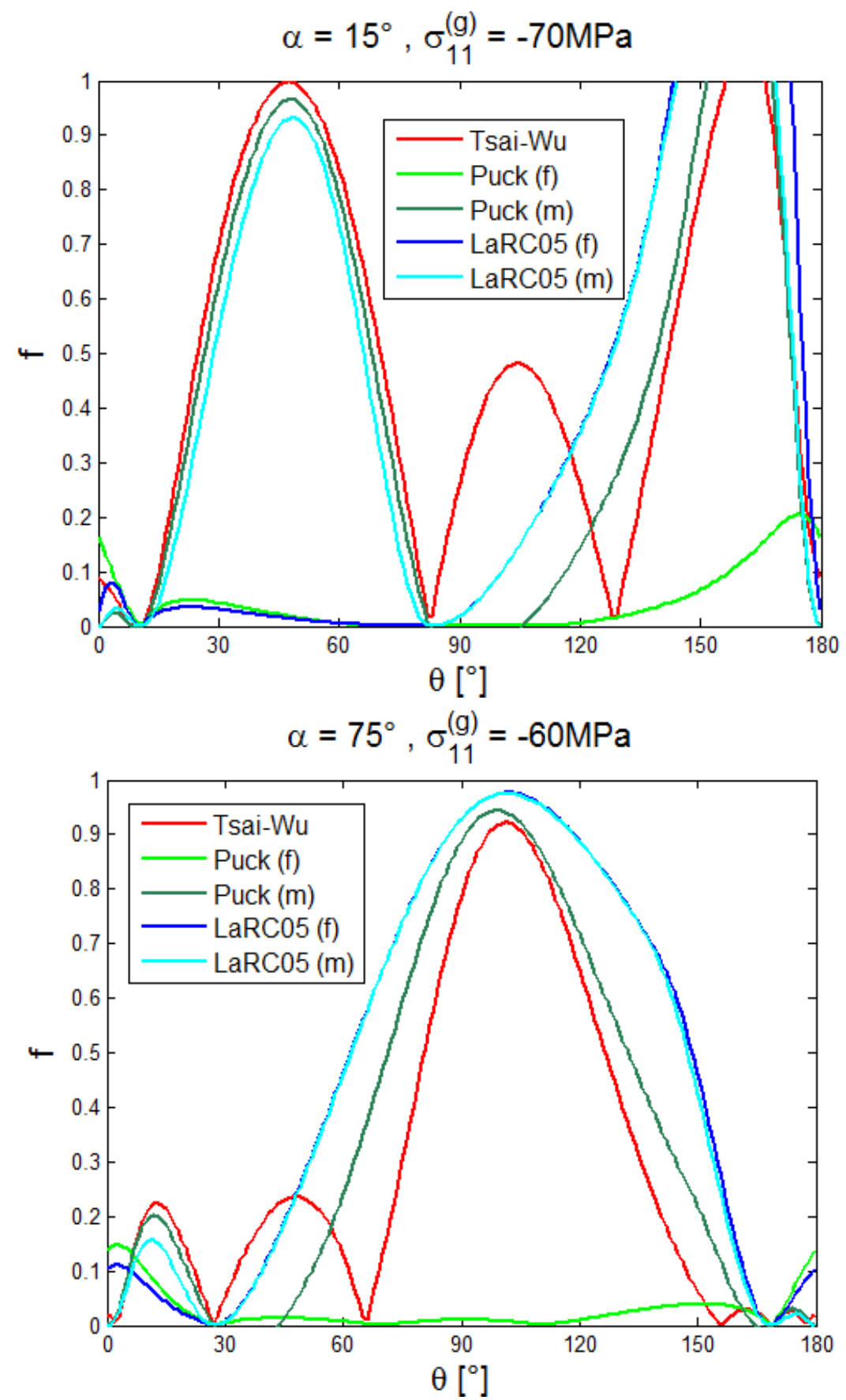

Figura 40 - Variação das funções de falha na borda do furo para carregamentos uniaxiais no laminado angle-ply com: $\sigma_{11}^{(g)}=90 M P a$ e $\alpha=0^{\circ} ; \sigma_{11}^{(g)}=-70 M P a$ e $\alpha=15^{\circ}$; e $\sigma_{11}^{(g)}=-60 M P a$ e $\alpha=75^{\circ}$ (continuação) 

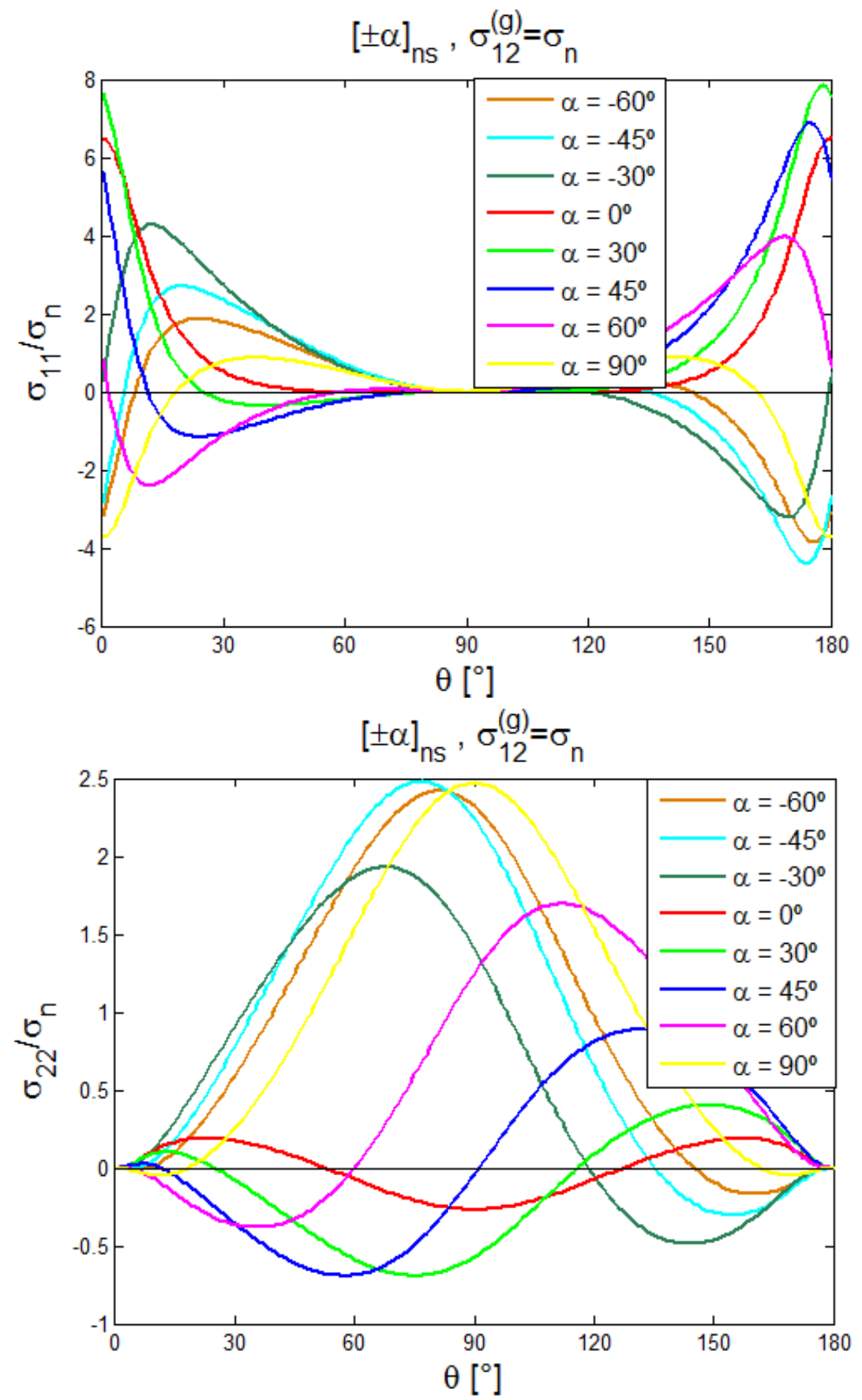

Figura 41 - Tensões ao longo da borda do furo em coordenadas do material para uma placa grande de laminado angle-ply com furo circular e carregada com cisalhamento puro (continua) 


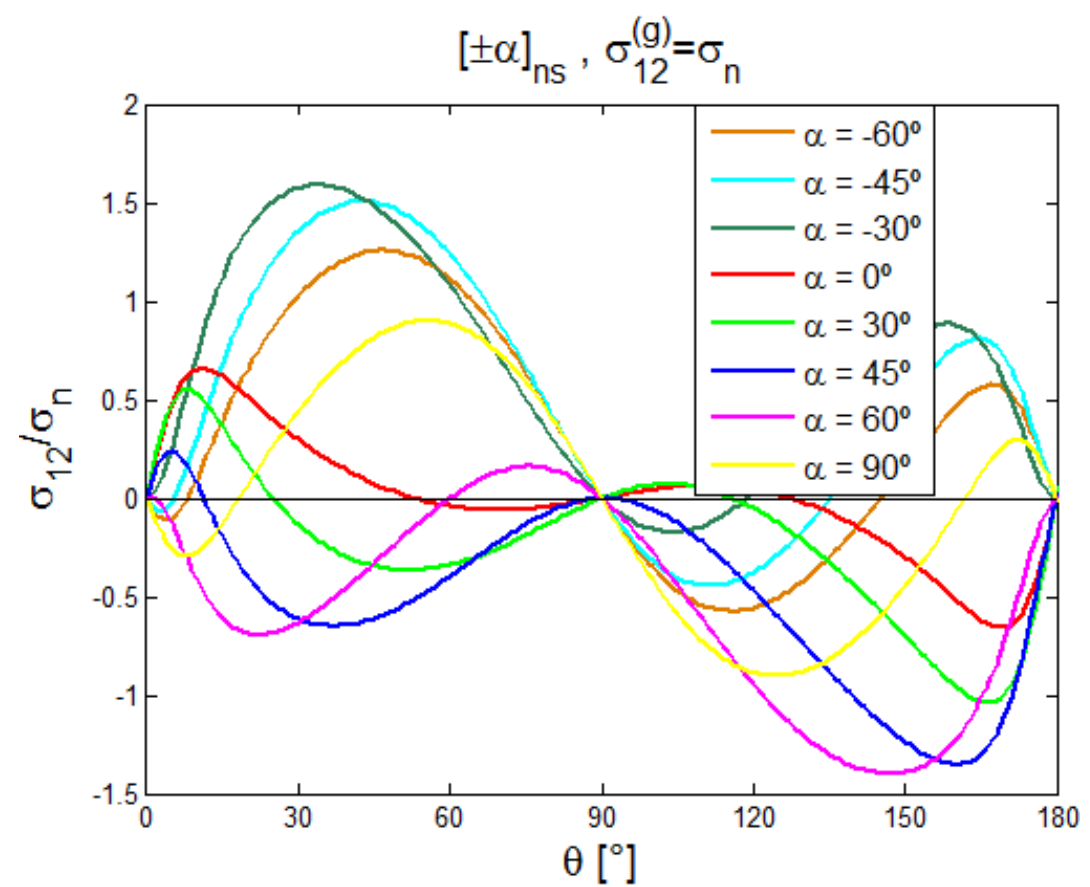

Figura 41 - Tensões ao longo da borda do furo em coordenadas do material para uma placa grande de laminado angle-ply com furo circular e carregada com cisalhamento puro (continuação)

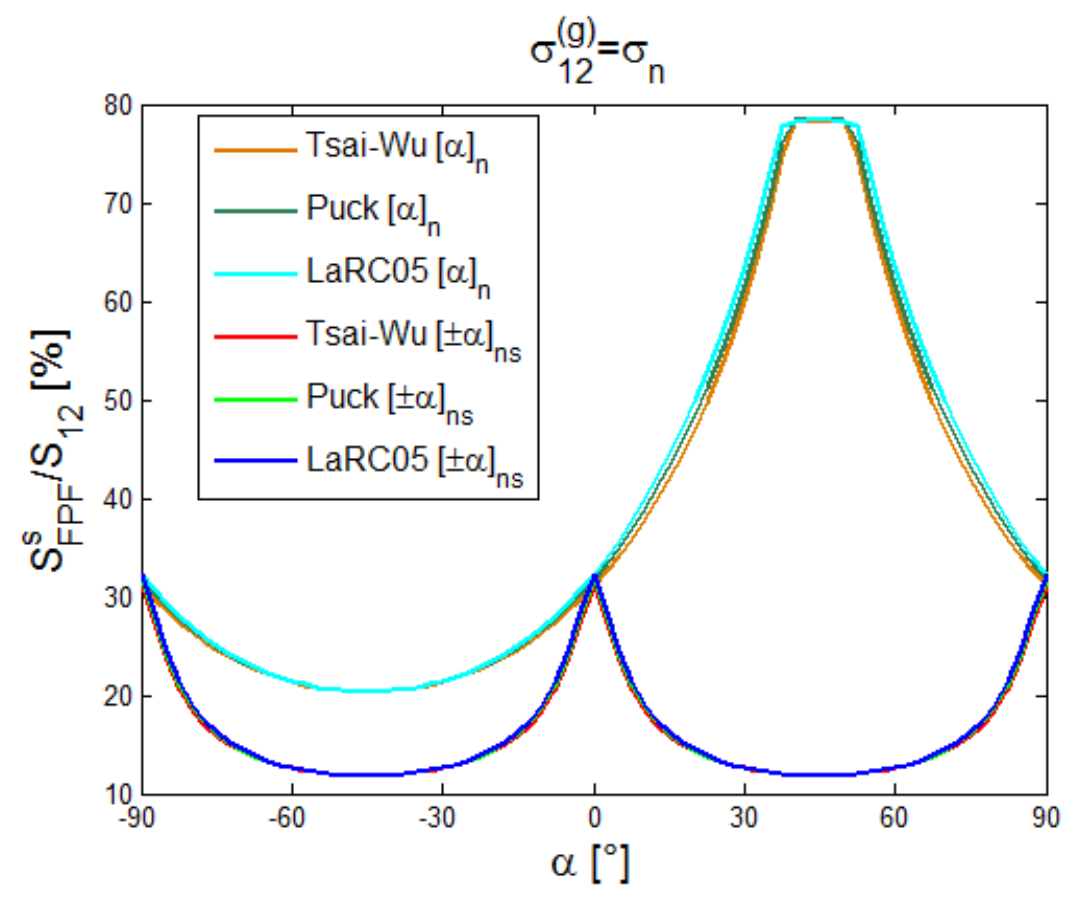

Figura 42 - Estimativas de $S_{F P F}^{s} / S_{12}$ para placas grandes de laminados angle-ply com furos circulares 
De uma forma geral, esse resultado pode ser entendido pela TCL; como não há o efeito do acoplamento entre tensões e deformações normais e cisalhantes, as matrizes de rigidez e de flexibilidade equivalentes têm uma forma similar, com relação aos elementos não nulos, as matrizes dos laminados unidirecionais para as fibras com inclinações $\alpha=0^{\circ}, \pm 90^{\circ}$, que são exatamente os casos em que a concentração de tensão é maior. Ou seja, os laminados angle-plies resultam em um estado de deformações equivalentes aos casos de laminados unidirecionais com $\alpha=0^{\circ}, \pm 90^{\circ}$, resultando então em um maior nível de tensões.

Como as tensões são maiores, é esperado que as resistências diminuam. A variação da resistência para o caso de cisalhamento aplicado é mostrada na Figura 42 e essa tendência pode ser observada, assim como para os casos de tração e compressão. Em especial para o cisalhamento, a curva deve ser simétrica em relação ao ponto em que $\alpha=0^{\circ}$. Como não há simetria para esse carregamento, $+\alpha$ e $-\alpha$ sofrem danos diferentes, como já mencionado, e a camada com maior dano controla a falha. Note que $[ \pm \alpha]_{n s}$ é equivalente a $[\mp \alpha]_{n s}$.

Para um angle-ply a condição ótima da inclinação das fibras em relação ao carregamento é $\alpha=0^{\circ}, \pm 90^{\circ}$, enquanto a melhor condição para laminados unidirecionais $\left(\alpha=45^{\circ}\right)$ representa a pior possível. Para placas sem entalhes, o desacoplamento entre efeitos cisalhantes e normais pode aumentar a resistência da peça, mas, pelo menos para a análise de início de dano, para placas entalhadas essa afirmação não é necessariamente verdadeira. Por outro lado, para a propagação do dano, é esperado que a resistência de angle-plies também seja maior do que de unidirecionais porque o dano é mais distribuído entre as camadas.

Para evidenciar a diferença entre camadas $+\alpha$ e $-\alpha$ que estejam no mesmo laminado sob um determinado carregamento, a variação das funções de falha na borda do furo são mostradas na Figura 43 para ambos, considerando uma tensão nominal de cisalhamento de $8.5 \mathrm{MPa}$ em um laminado $[ \pm 45]_{n s}$. Enquanto todos os modelos indicam a falha para as lâminas com orientação $\alpha=-45^{\circ}$, o valor máximo estimado pelos modelos para as camadas com inclinação $\alpha=45^{\circ}$ é perto de 0.5 , resultando em danos completamente diferentes. 

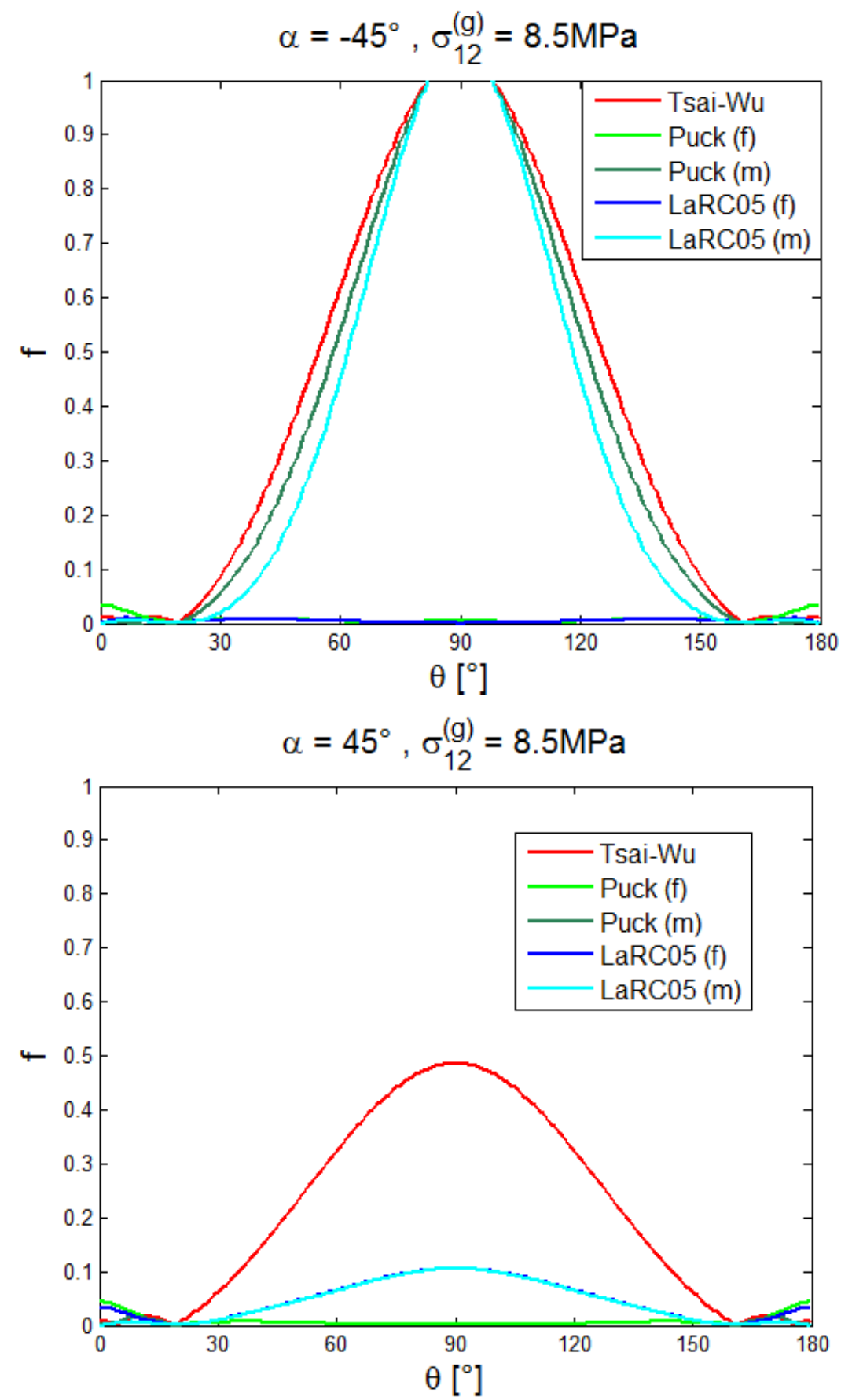

Figura 43 - Variação das funções de falha na borda do furo para carregamentos cisalhantes de $\sigma_{12}^{(g)}=8.5 \mathrm{MPa}$ no laminado angle-ply para lâminas com $\alpha= \pm 45^{\circ}$ 


\subsection{Estimativas de resistências para laminados $[\alpha]_{n}$ com furo elíptico}

Furos elípticos necessitam de duas variáveis adicionais para serem descritos: a razão entre os semi-eixos $\left(r_{a} / r_{b}\right)$ e a inclinação da elipse $(\beta)$, o que resulta em uma análise mais trabalhosa. Algumas vezes se torna útil plotar os resultados usando a seguinte definição para a inclinação da elipse: $\beta^{*}=\beta+\alpha$, que é a inclinação da elipse em relação aos eixos globais que definem o carregamento, ao invés de definir a inclinação em relação às coordenadas do material. Apesar de mais elaborados, furos elípticos são especialmente úteis para aproximar outros entelhas sem solução analítica conhecida e estudos sobre gradientes de tensões e mecânica da fratura (INGLIS, 1913 e GRIFFITH, 1921). Três diferente razões dos semi-eixos serão estudadas $\left(r_{a} / r_{b}=2,5,10\right)$ e apenas carregamentos uniaxiais serão considerados nessa etapa.

As Figuras 44-46 mostram as componentes das tensões (em coordenadas do material) para vários laminados unidirecionais considerando a elipse definida por $r_{a} / r_{b}=2$. Para uma melhor e mais eficiente intepretação dos resultados, cada componente de tensão é mostrada em uma mesma escala de cor para todos os valores de $\alpha$.
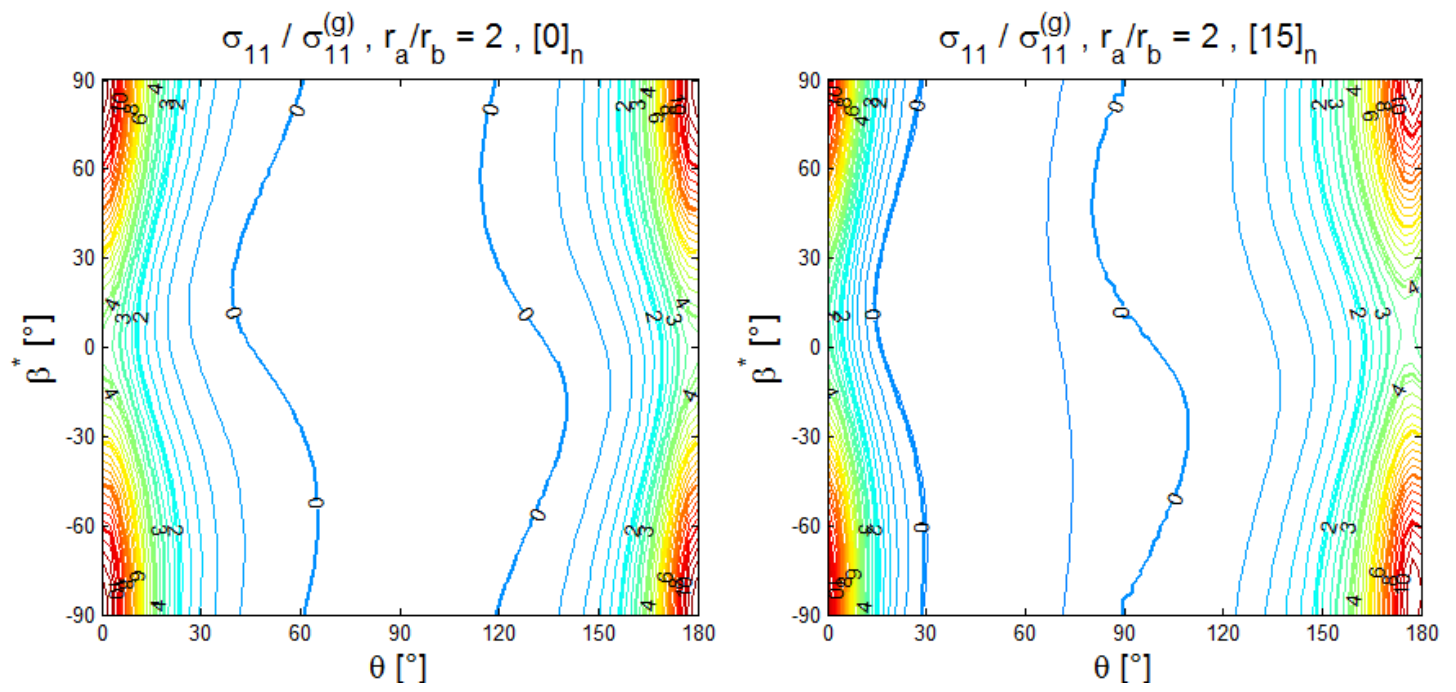

Figura 44 - Variação de $\sigma_{11}$ ao longo da borda do furo placa grande de laminados unidirecionais com furo elítico $\left(r_{a} / r_{b}=2\right)$ e carregada uniaxialmente (continua) 

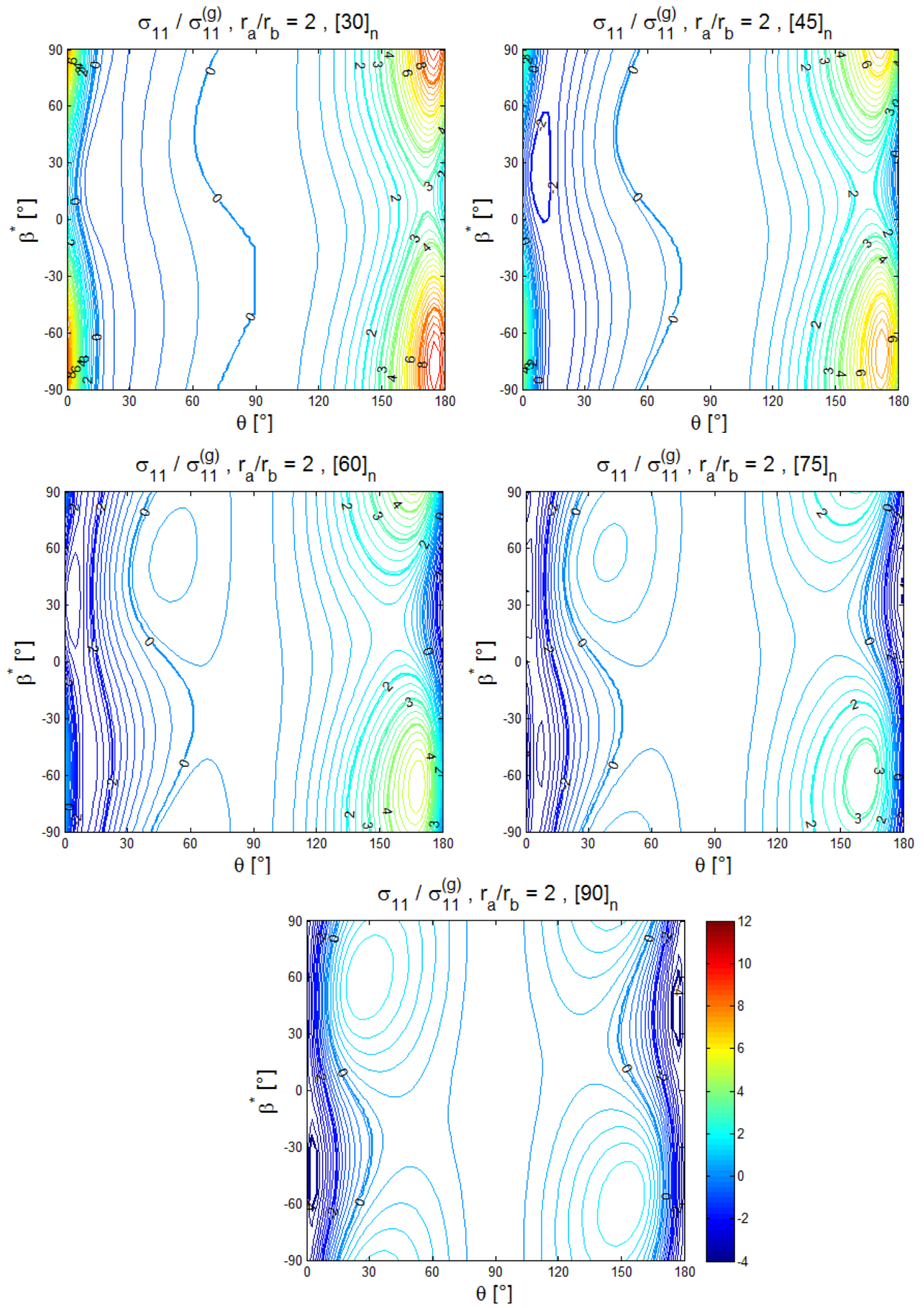

Figura 44 - Variação de $\sigma_{11}$ ao longo da borda do furo placa grande de laminados unidirecionais com furo elítico $\left(r_{a} / r_{b}=2\right)$ e carregada uniaxialmente (continuação) 
A Figura 43 mostra que $\sigma_{11}$ é maximizado quando $\alpha=0^{\circ}$, o que é o oposto do que acontece com $\sigma_{22}$, que tem seu valor máximo para $\alpha=90^{\circ}$. O mais importante resultado dessas figuras é que a inclinação da elipse que maximiza a concentração de tensão em módulo não é necessariamente para $\beta^{*}=90^{\circ}$, como é sabido para materiais isotrópicos. $\sigma_{11}$ é maximizado quando $90^{\circ}<\left|\beta^{*}\right|<90^{\circ}+\alpha$ (ou seja, $90^{\circ}-\alpha<|\beta|<90^{\circ}$ ), como pode ser claramente percebido para $[30]_{n}$ na Figura 43 , e $\sigma_{22}$ tem o seu valor absoluto máximo quando $60^{\circ}<\left|\beta^{*}\right|<90^{\circ}$ (ou $60^{\circ}-\alpha<|\beta|<90^{\circ}-\alpha$ ), e.g. para $[60]_{n}$ na Figura 44. Apesar de $\sigma_{12}$ também influenciar na falha, $\sigma_{11}$ e $\sigma_{22}$ desempenham um papel de compreensão física mais direta uma vez que a influência dos mesmos é normalmente separada na falha da matriz ou da fibra, enquanto $\sigma_{12}$ influencia em ambas. Note que não é viável plotar os resultados em gráficos 2D pelo grande número de variáveis.
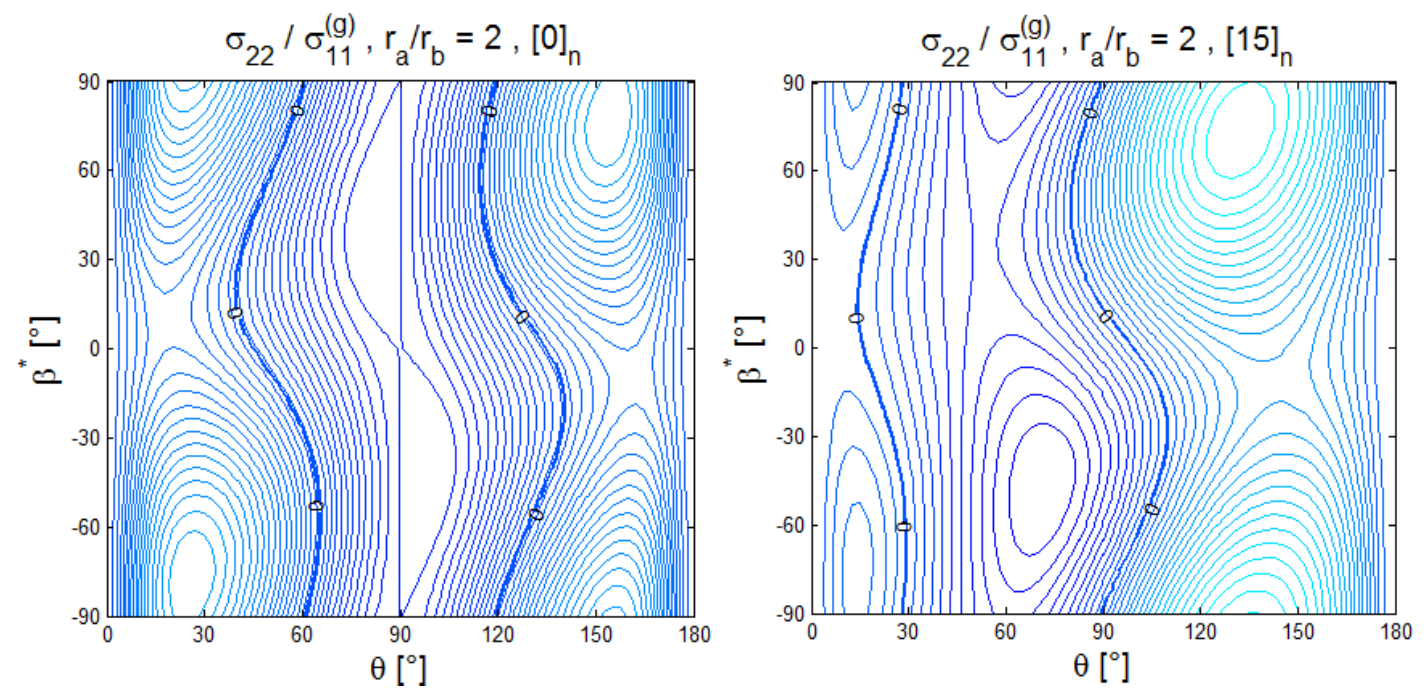

Figura 45 - Variação de $\sigma_{22}$ ao longo da borda do furo placa grande de laminados unidirecionais com furo elítico $\left(r_{a} / r_{b}=2\right)$ e carregada uniaxialmente (continua) 

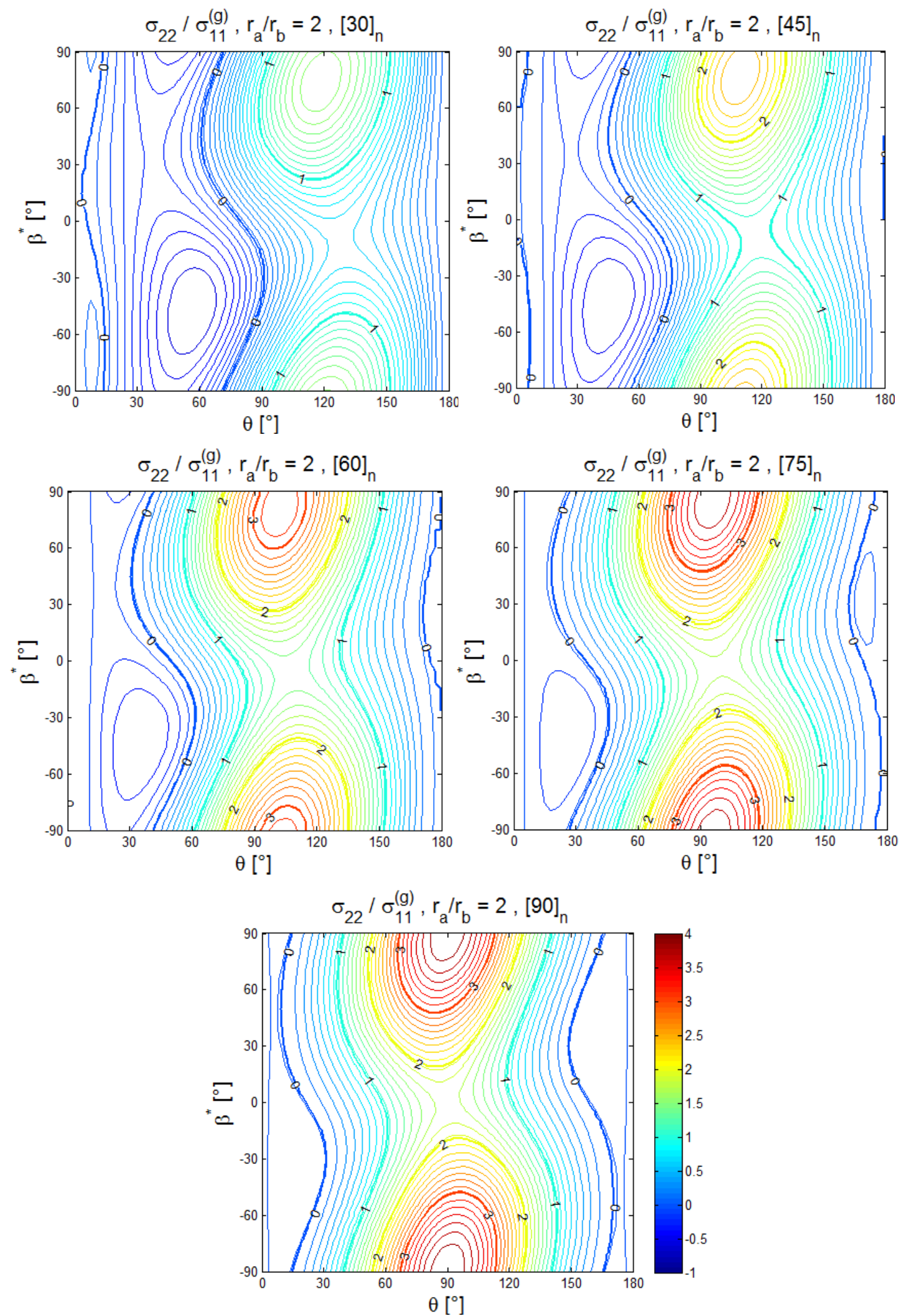

Figura 45 - Variação de $\sigma_{22}$ ao longo da borda do furo placa grande de laminados unidirecionais com furo elítico $\left(r_{a} / r_{b}=2\right)$ e carregada uniaxialmente (continuação) 

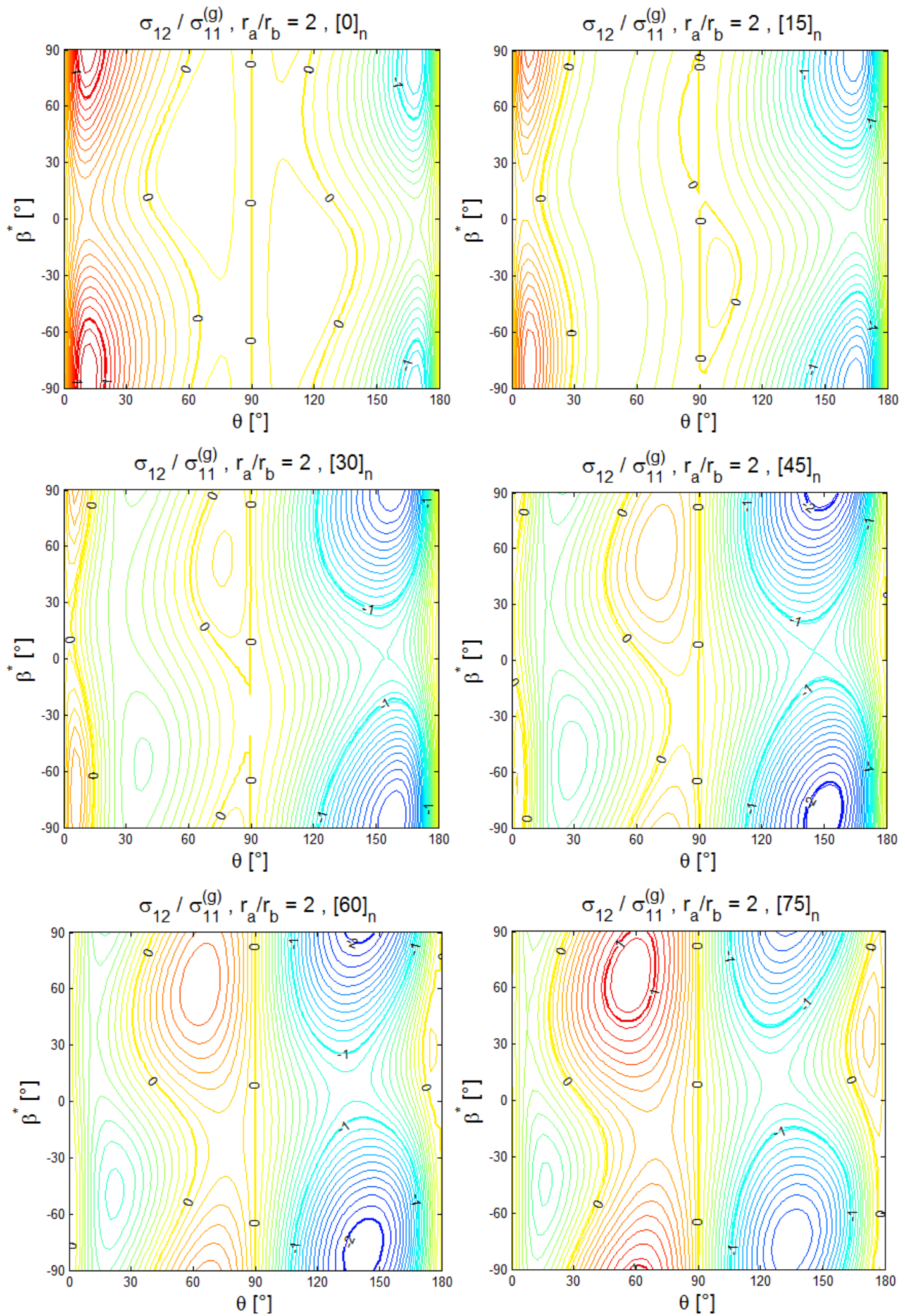

Figura 46 - Variação de $\sigma_{12}$ ao longo da borda do furo placa grande de laminados unidirecionais com furo elítico $\left(r_{a} / r_{b}=2\right)$ e carregada uniaxialmente (continua) 


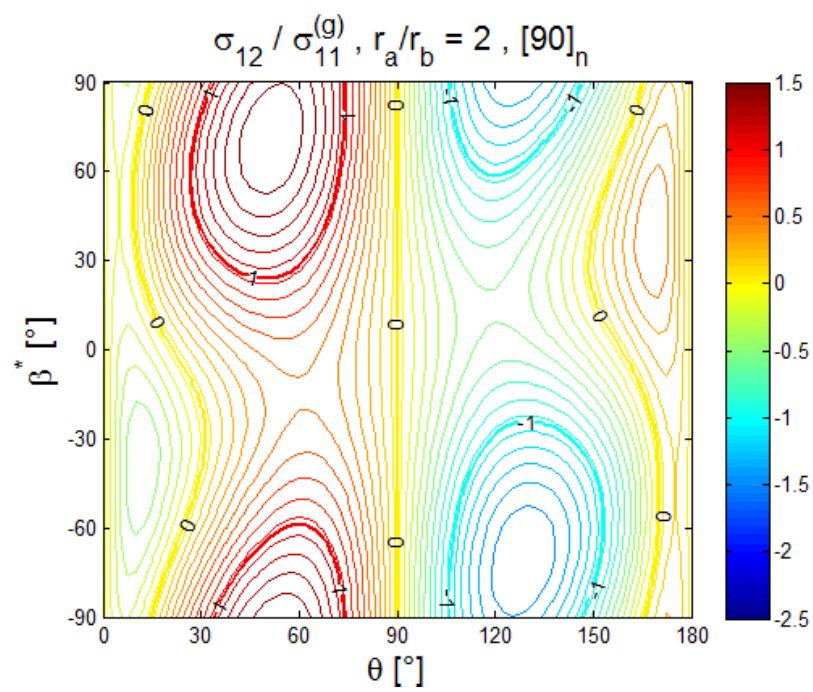

Figura 46 - Variação de $\sigma_{12}$ ao longo da borda do furo placa grande de laminados unidirecionais com furo elítico $\left(r_{a} / r_{b}=2\right)$ e carregada uniaxialmente (continuação)

Hwu e Ting (1989) provaram analiticamente que para carregamentos uniaxiais $\sigma_{11}^{(g)}=\sigma_{n}$ e inclinação da elipse $\beta=90^{\circ}$, a concentração de tensão na borda do furo sobre o menor semi-eixo da elipse $\left(\theta=0^{\circ}\right)$, que para materiais isotrópicos é igual à -1 , para materiais anisotrópicos também é independente da geometria do furo, depende apenas das propriedades do material. Todavia, apesar da máxima tensão definir a falha para materiais isotrópicos, para materiais anisotrópicos é necessário avaliar toda a distribuição de tensão na borda do furo, como já feito anteriormente.

Para avaliar a influência da inclinação do furo na falha, assim como a influência da razão entre os semi-eixos, um estudo paramétrico para carregamento trativos e compressivos foi desenvolvido para estimar as suas respectivas resistências e os resultados são mostrados nas Figuras 46 e 47. Note que as escalas são iguais para todos os gráficos que mostram a resistência à compressão e o mesmo não é verdade para os resultados das resistências à tração; escalas diferentes foram utilizadas no eixo vertical para possibilitar uma boa interpretação de todos os gráficos. Essa observação indica que a resistência à tração é mais sensível ao valor de $r_{a} / r_{b}$.

Para tensão, $\beta=-\alpha$, que significa que o maior eixo da elipse é paralelo à direção do carregamento, é a posição que resulta em menor dano, ou em outras palavras, a posição de elipse em que a placa tem uma maior resistência, concordando que a tradicional abordagem 
feita para materiais isotrópicos. Por outro lado, a posição mais crítica de elipse está no intervalo $90^{\circ}-\alpha<\beta<90^{\circ}$, ou seja, o maior eixo da elipse entre $x_{2}^{(g)}$ e $x_{2}$. Logo, se $\alpha=0^{\circ}$, há uma tendência do dano progredir perpendicularmente ao carregamento e às fibras (que para esse caso têm a mesma direção) o que pode ser visto pela Figura 46 que, principalmente para valores maiores de $r_{a} / r_{b}$, resultando uma falha final dominada pela ruptura das fibras. Para $\alpha=90^{\circ}$, é esperado que a matriz falhe, como claramente mostrado pela diferença dos valores das resistências. Pela grande diferença entre os valores das resistências estimadas, é esperado que a falha final seja dominada pela matriz pelo menos para $\alpha>15^{\circ}$, o que está de acordo com os resultados apresentados por Kaman (2011).

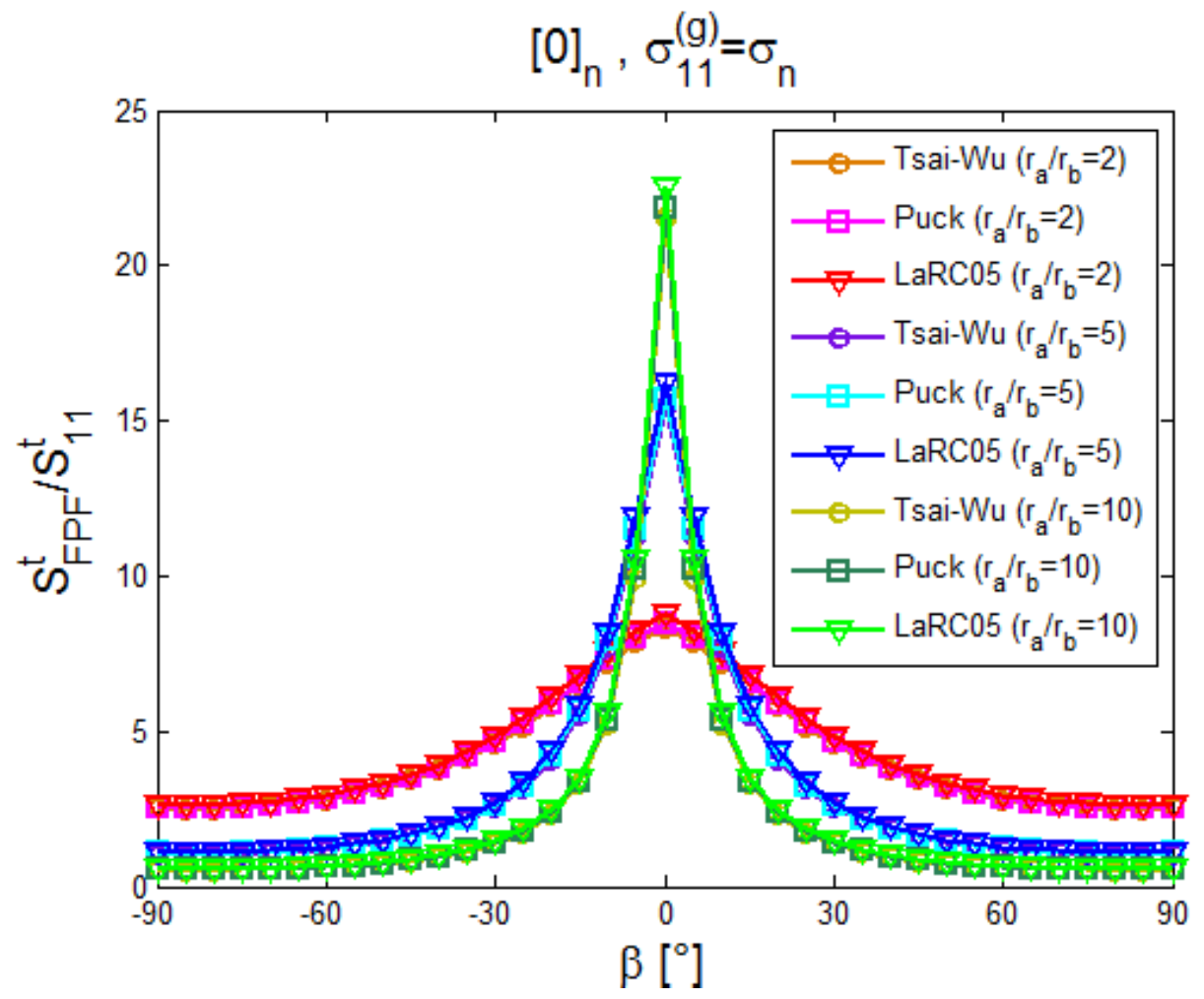

Figura 47 - Estimativas de $S_{F P F}^{t} / S_{11}^{t}$ para placas grandes de laminados unidirecionais com furos elípticos (continua) 

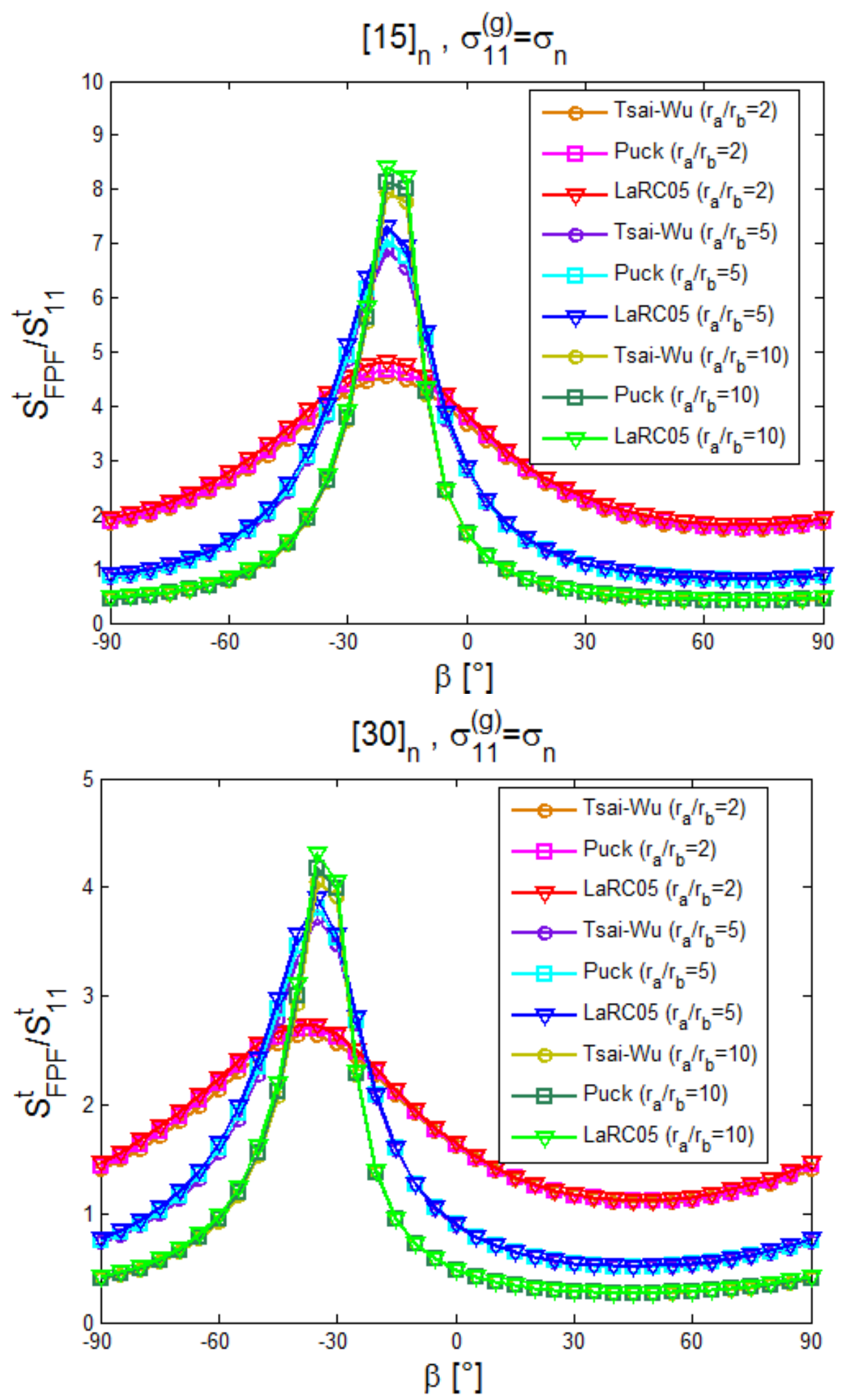

Figura 47 - Estimativas de $S_{F P F}^{t} / S_{11}^{t}$ para placas grandes de laminados unidirecionais com furos elípticos (continua) 

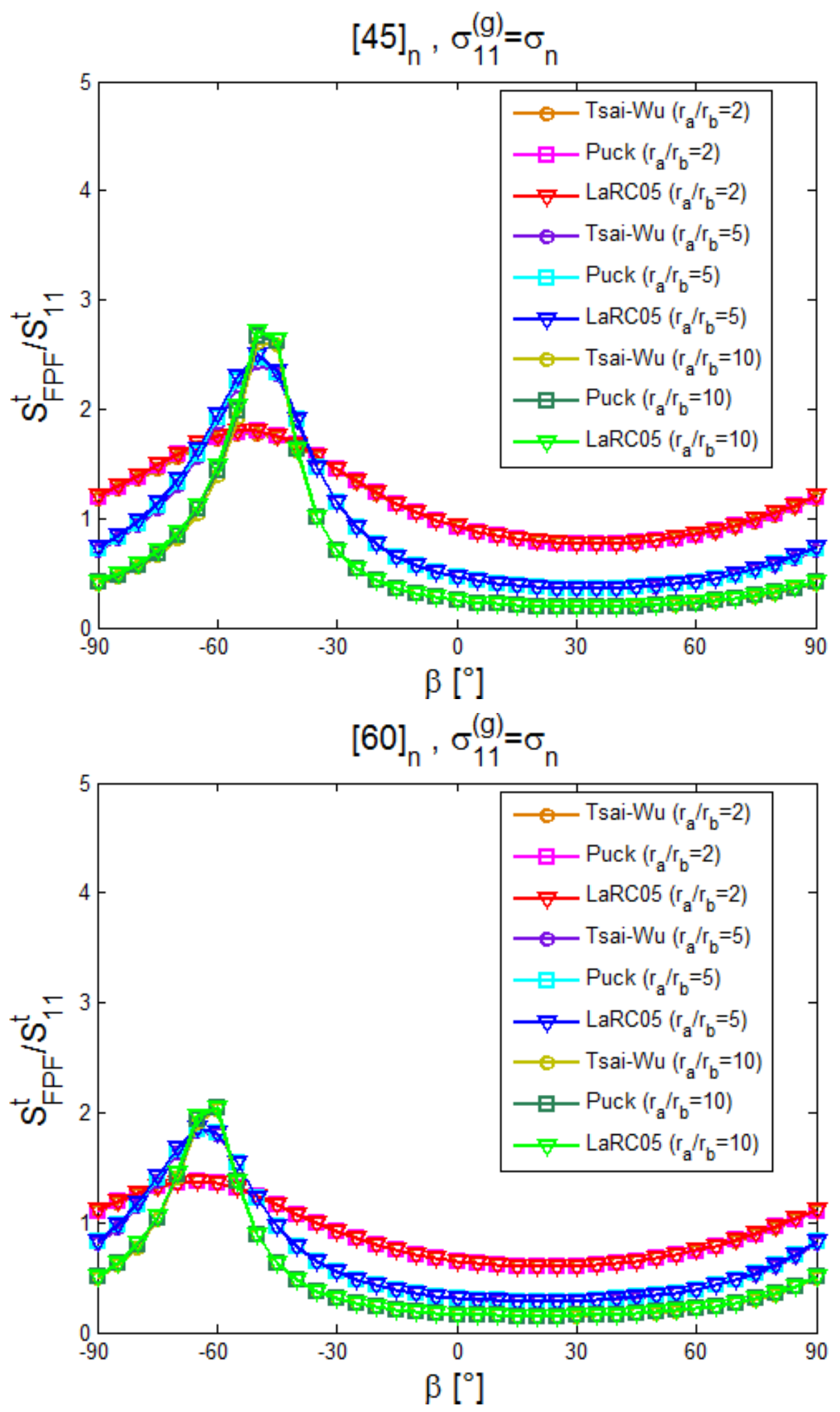

Figura 47 - Estimativas de $S_{F P F}^{t} / S_{11}^{t}$ para placas grandes de laminados unidirecionais com furos elípticos (continua) 

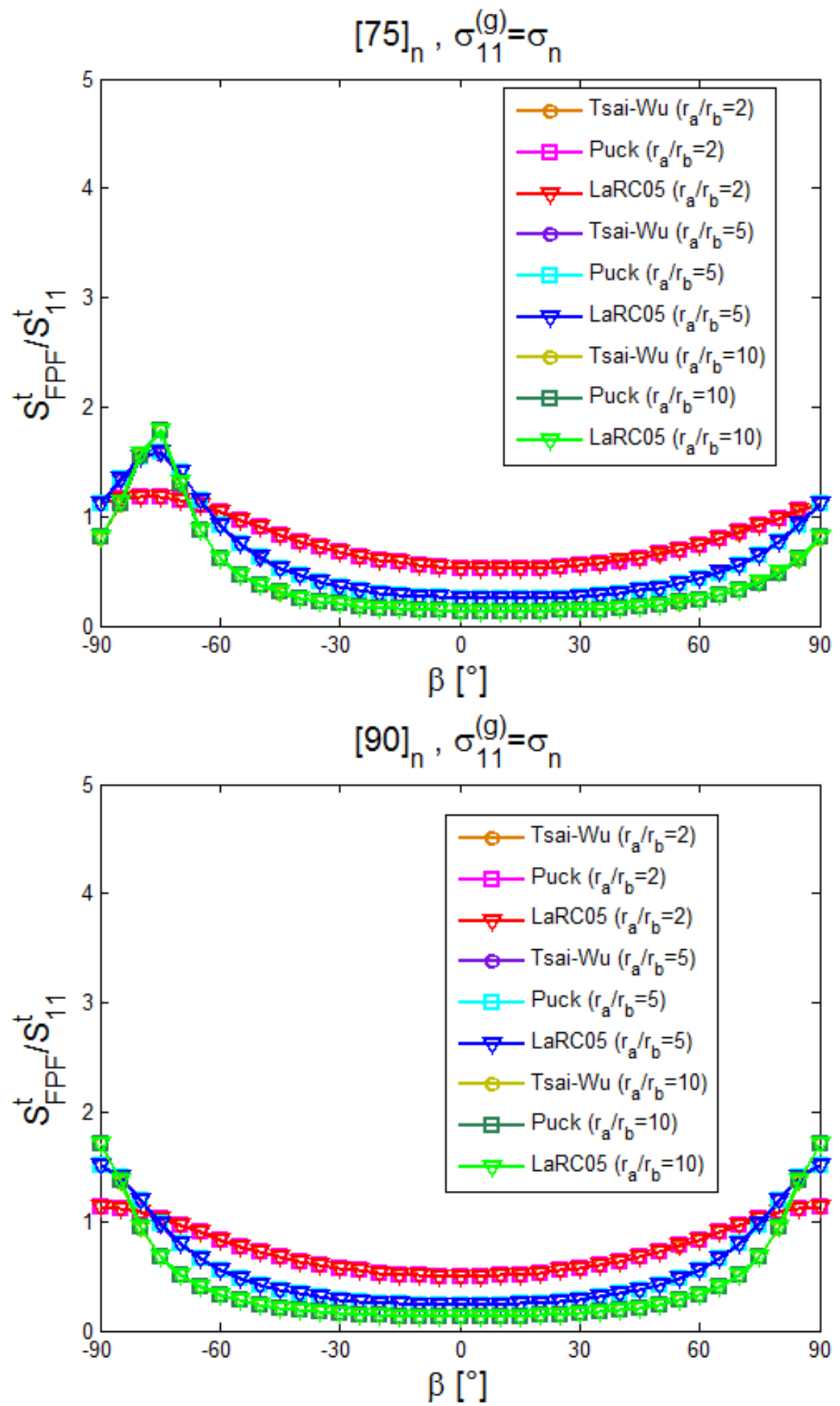

Figura 47 - Estimativas de $S_{F P F}^{t} / S_{11}^{t}$ para placas grandes de laminados unidirecionais com furos elípticos (continuação) 
Para a resistência à compressão, a tendência de LaRC05 indicar falha, por causa da fibra sobre compressão, continua existindo mas diminui quando a razão $r_{a} / r_{b}$ aumenta. Isso se deve ao fato de que quanto maior o valor de $r_{a} / r_{b}$, mais o ponto crítico se aproxima do ponto sobre o maior semi-eixo (menor raio da elipse). Por exemplo, para o caso extremo em que a elipse tende a se tornar uma trinca, o ponto crítico sempre será na ponta da trinca pela singularidade das tensões. Logo, a fibra só irá falhar se estiver perpendicular ao maior eixo da elipse.

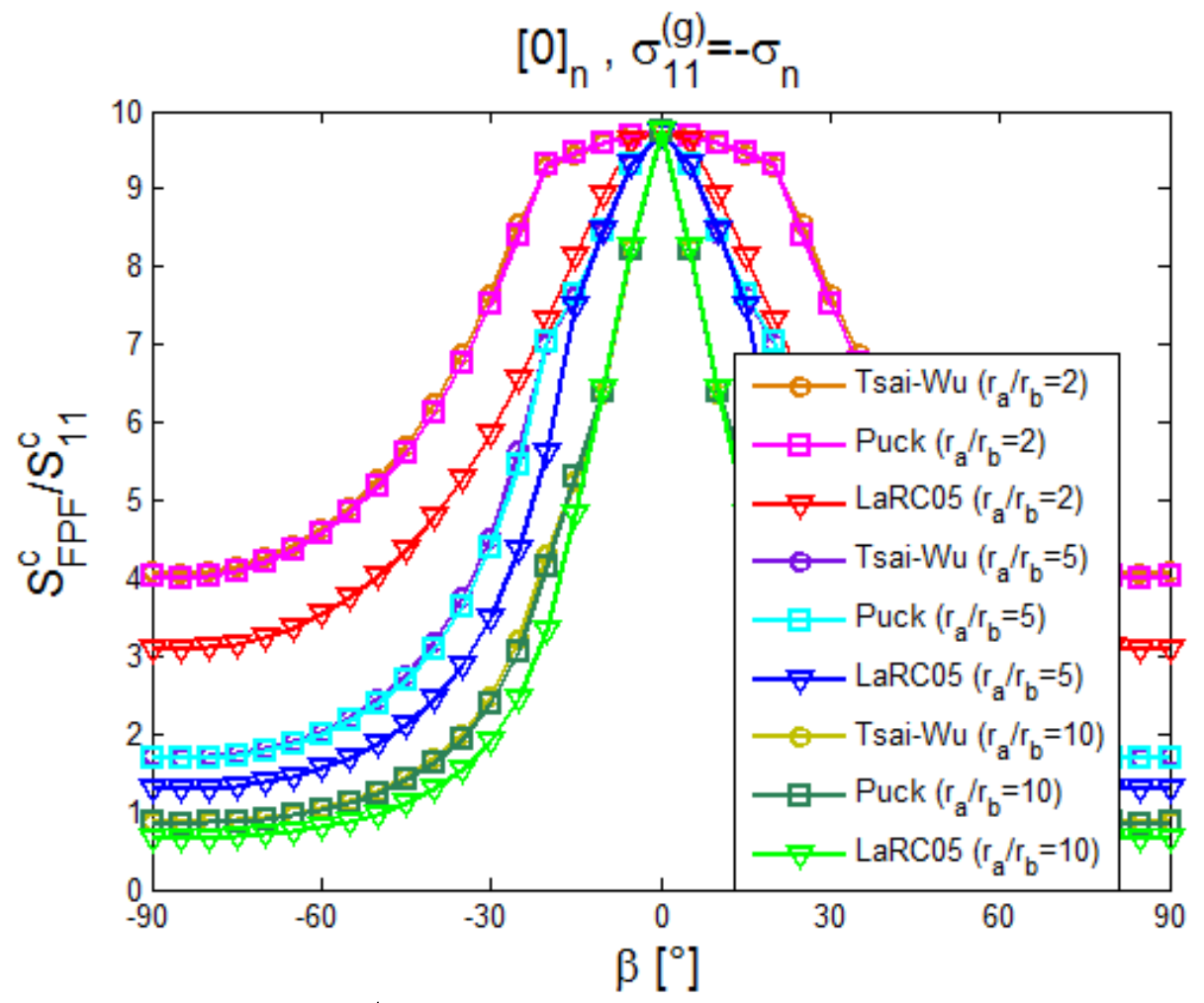

Figura 48 - Estimativas de $S_{F P F}^{c} / S_{11}^{c}$ para placas grandes de laminados unidirecionais com furos elípticos (continua) 

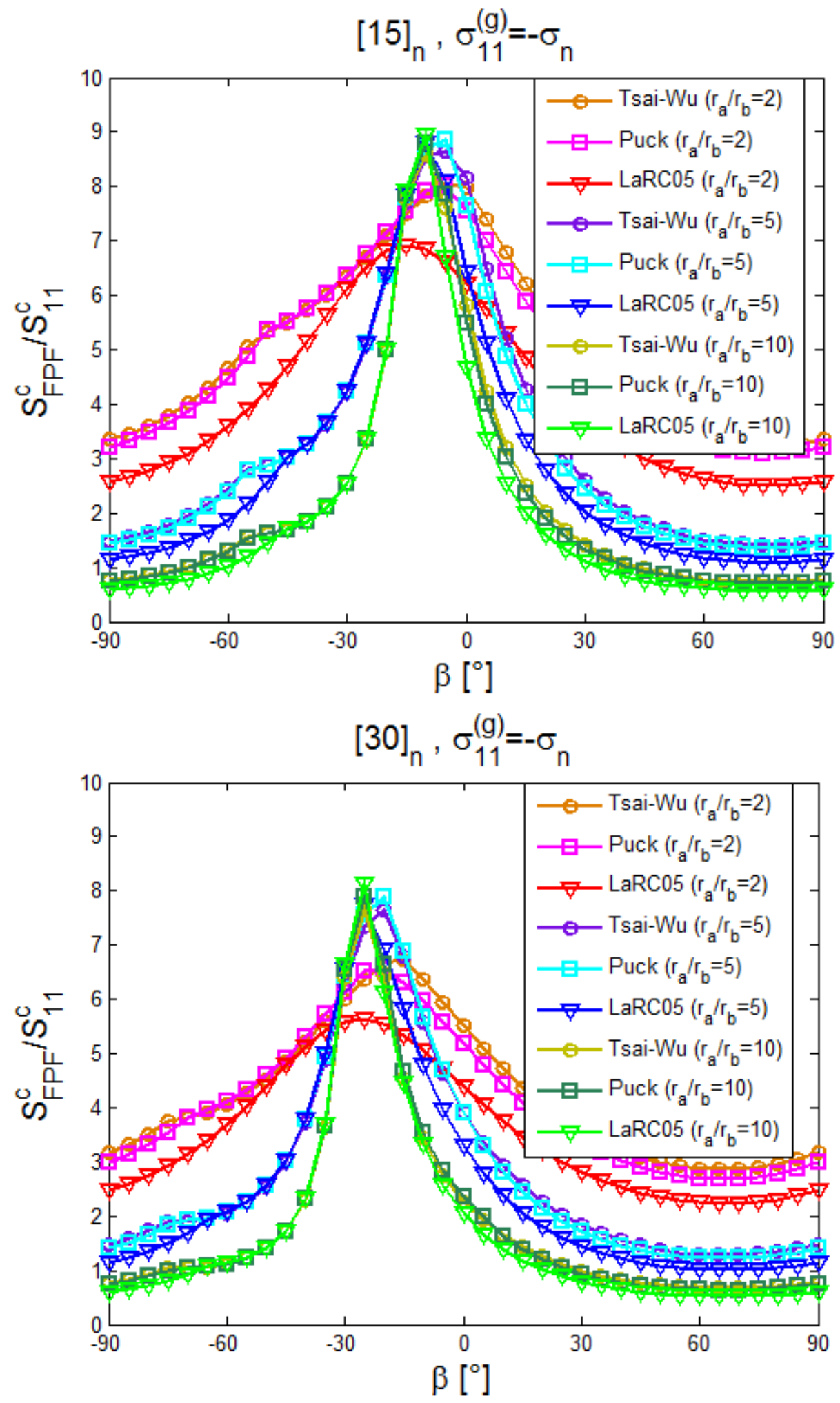

Figura 48 - Estimativas de $S_{F P F}^{c} / S_{11}^{c}$ para placas grandes de laminados unidirecionais com furos elípticos (continua) 

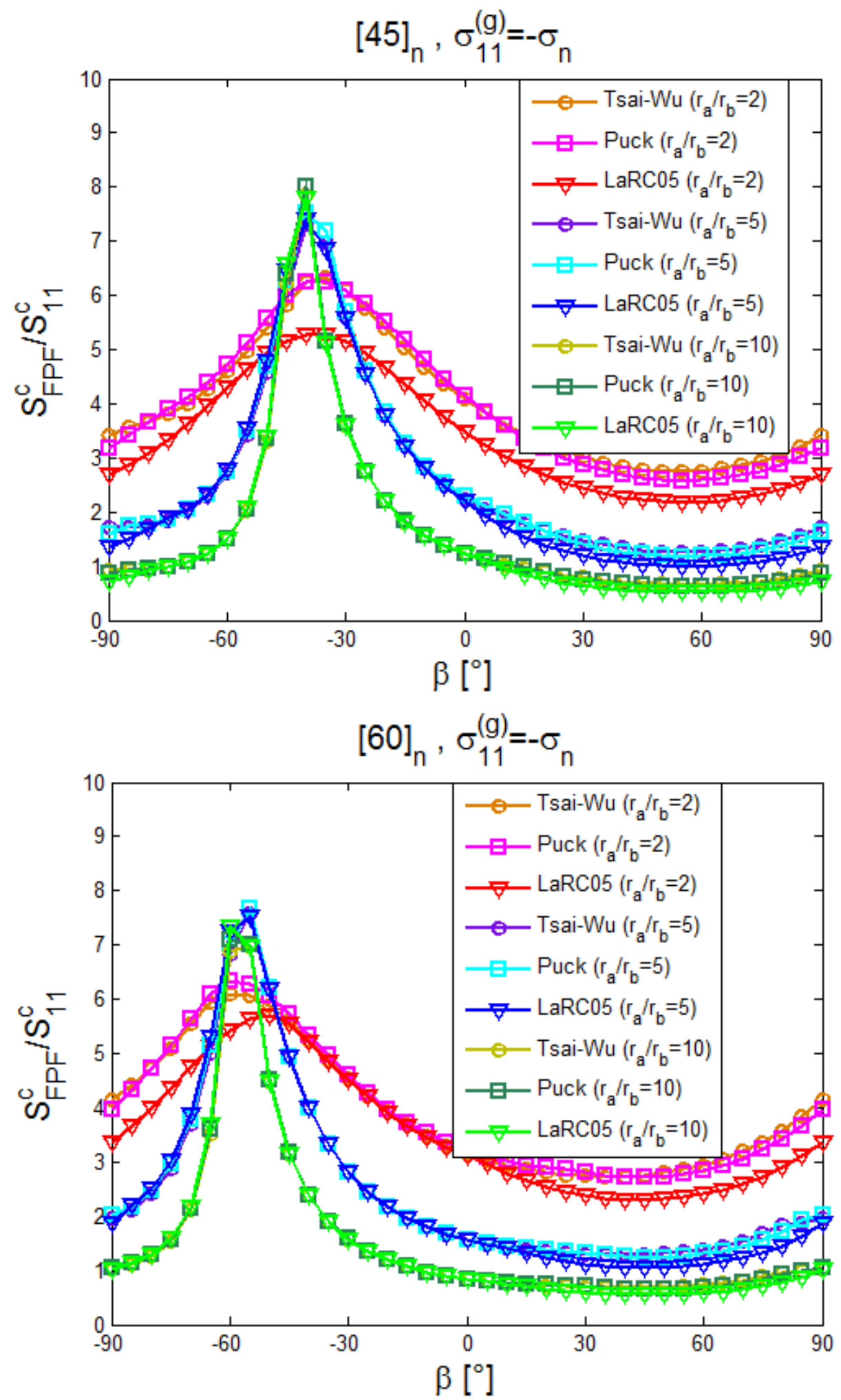

Figura 48 - Estimativas de $S_{F P F}^{c} / S_{11}^{c}$ para placas grandes de laminados unidirecionais com furos elípticos (continua) 

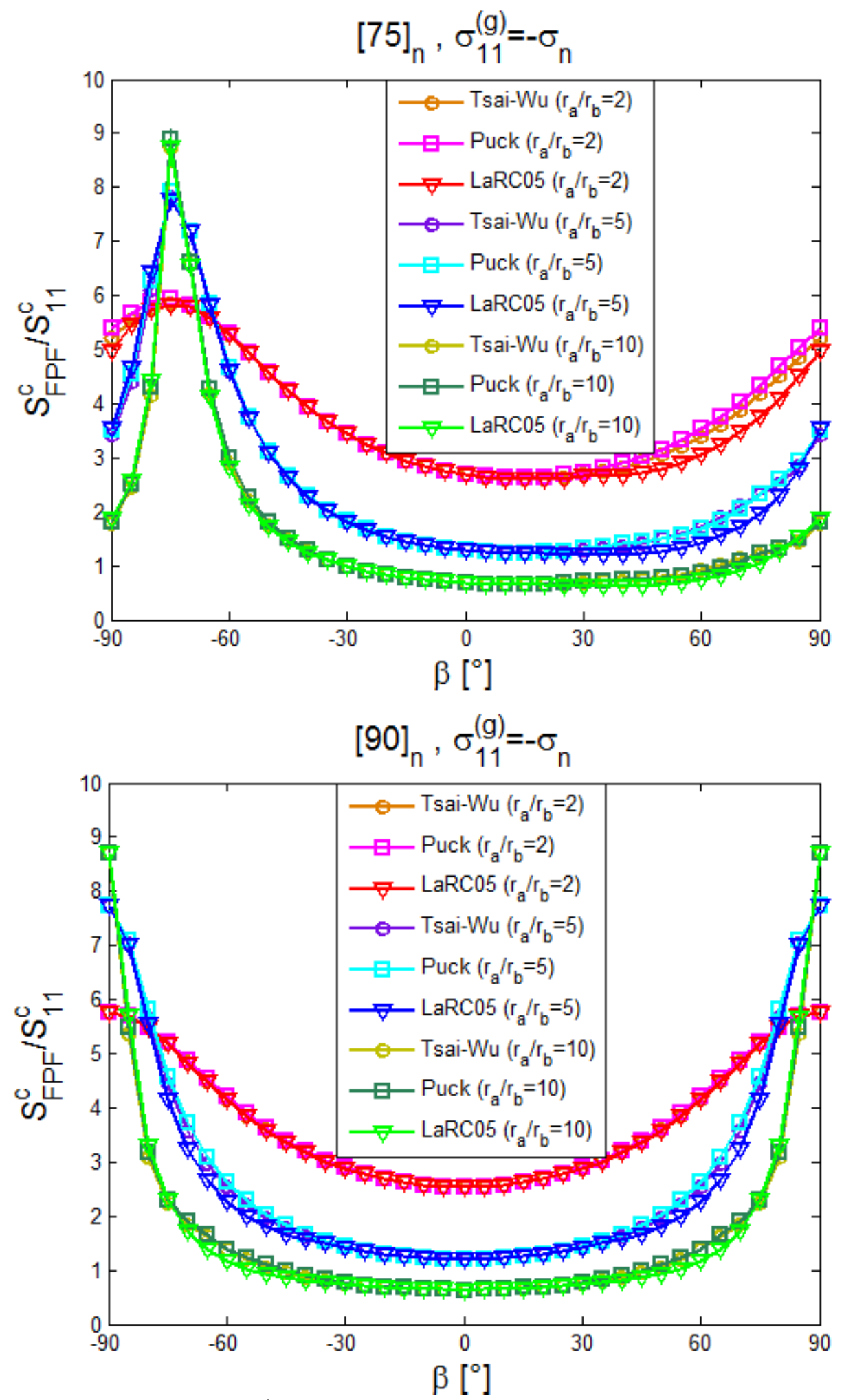

Figura 48 - Estimativas de $S_{F P F}^{c} / S_{11}^{c}$ para placas grandes de laminados unidirecionais com furos elípticos (continuação) 
Pela Figura 46 é possível perceber que para $[0]_{n}$, quando $r_{a} / r_{b}$ aumenta, maior é a tensão nominal aplicada necessária para a falha se $\beta=0^{\circ}$ e consideravelmente menor é a tensão necessária para $\beta= \pm 90^{\circ}$, mesmo para menores razões $r_{a} / r_{b}$. Então, para um furo circular, é esperado que a falha se inicie na região perto de $\theta=15^{\circ}$ (ver Figura 27) e a sua progressão tenda a torná-la perpendicular às fibras $\left(\theta=0^{\circ}\right)$, resultando em uma falha final dominantemente controlada pelas fibras. Para $[90]_{n}$, um desenvolvimento oposto pode ser desenvolvido, resultando em uma falha dominada pela matriz.

As Figuras 49 e 50 mostram resultados retirados da literatura e mostram alguns tipos de falha para ilustrar o que foi discutido. Na Figura 49 são mostrados resultados que indicam que a falha se inicia em pontos diferentes de diversos tipos de falha final e na Figura 50 são mostradas as progressões do dano para várias lâminas com orientações diferentes das fibras. Nessa última, inicialmente o laminado foi comprimido e depois tracionado, ambos uniaxialmente, sendo as imagens de lâminas diferentes (cada uma com uma orientação) do mesmo laminado.
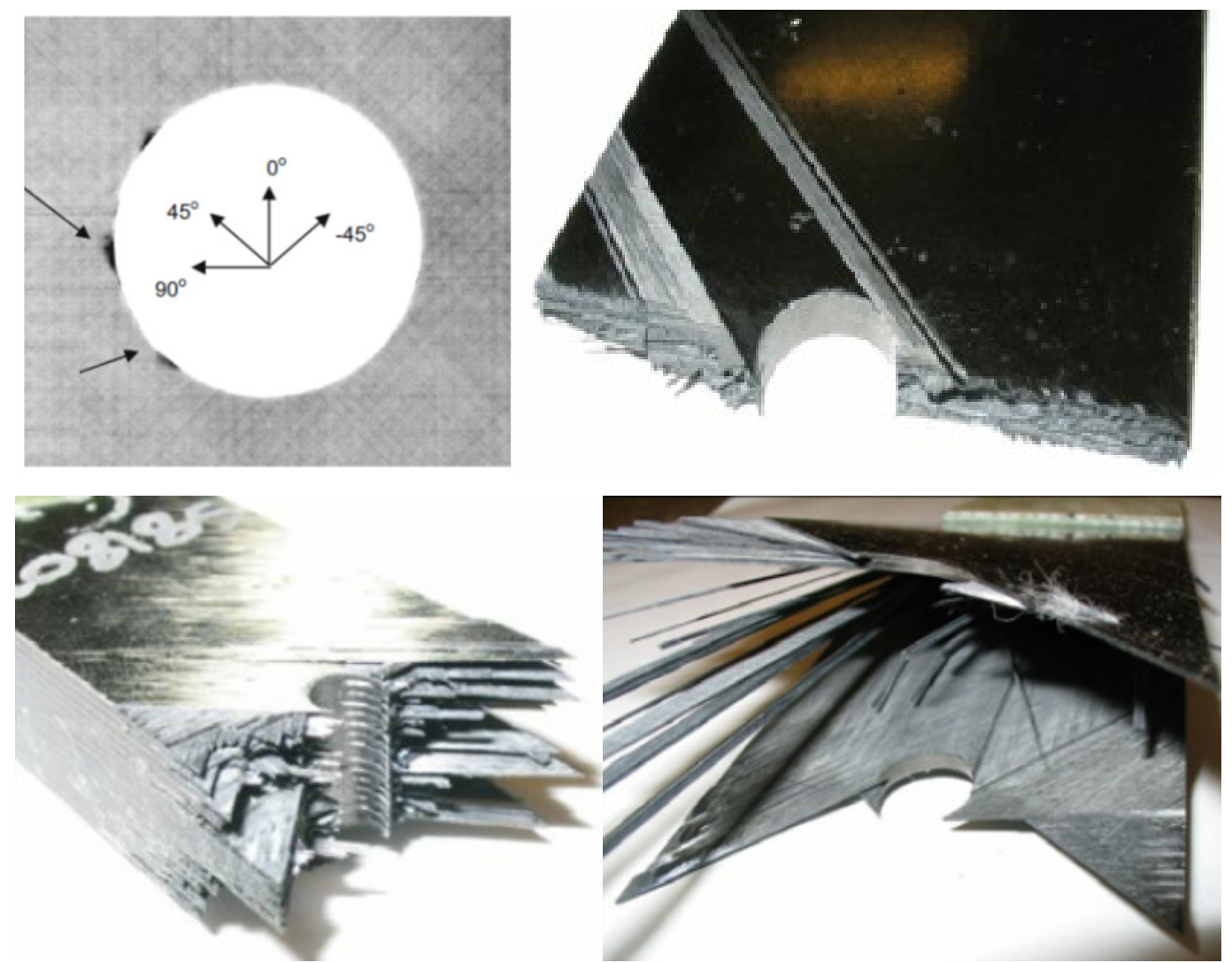

Figura 49 - Diferentes tipos de falhas de um laminado: (a) início do dano em regiões diferentes; (b) ruptura frágil; (c) pull-out; (d) delaminação (adaptada de Hallett et al., 2009). 

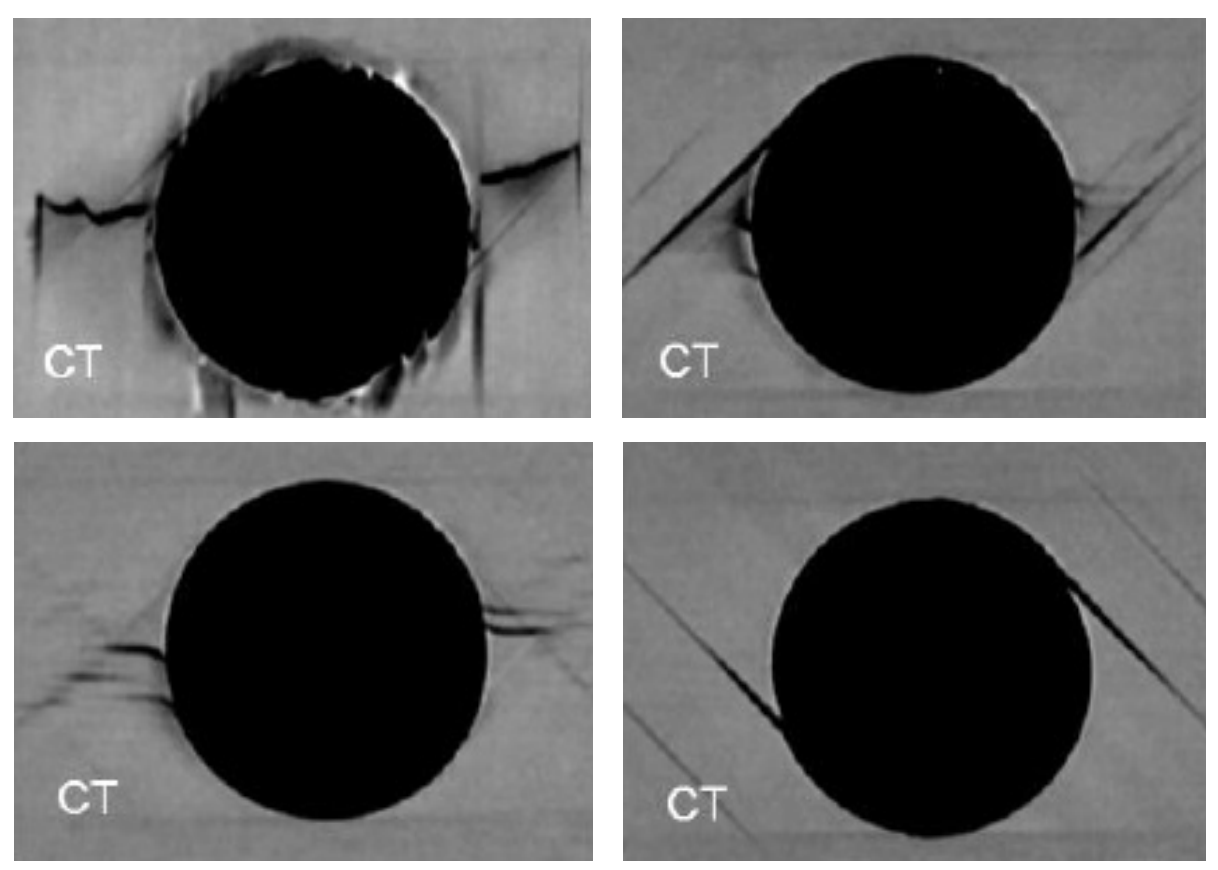

Figura 50 - Diferentes modos de progressão da falha em uma lâmina com furo circular de acordo com a direção de laminação: (a) $0^{\circ}$; (b) $45^{\circ}$; (c) $90^{\circ}$; (d) $-45^{\circ}$ (adaptada de Nikishkov et al., 2015) 


\section{Efeito de furos em placas sob deformação plana}

Apesar do efeito da espessura normalmente não ser considerado em problemas de concentração de tensão mesmo para materiais isotrópicos, Góes et al. (2014) apresentaram resultados que mostram a influência da geometria 3D na distribuição de tensões ao redor do entalhe. Baseado nessas conclusões, é esperado que materiais anisotrópicos também sofram essa influência na distribuição de tensões e principalmente nas estimativas de falha. Para quantificar esse efeito será utilizado o fator de restrição transversal definido por (GÓES et al., 2014)

$$
T_{c}=\frac{\sigma_{33}^{(l)}}{\sigma_{11}^{(l)}+\sigma_{22}^{(l)}}
$$

Note que o mesmo é definido em relação às coordenadas locais. A relação constitutiva de uma forma genérica pode ser escrita para as coordenadas locais como

$$
\varepsilon_{i j}^{(l)}=c_{i j k m}^{(l)} \sigma_{k m}^{(l)}
$$

Note que $c_{i j k m}^{(l)}$ é o tensor de flexibilidade em coordenadas locais. Para as coordenadas do material, o tensor 3D de flexibilidade pode ser definido de forma matricial como (LEKHNISKII, 1981)

$$
\boldsymbol{c}=\left[\begin{array}{cccccc}
\frac{1}{E_{1}} & -\frac{\nu_{12}}{E_{1}} & -\frac{\nu_{13}}{E_{1}} & 0 & 0 & 0 \\
-\frac{\nu_{21}}{E_{2}} & \frac{1}{E_{2}} & -\frac{\nu_{23}}{E_{2}} & 0 & 0 & 0 \\
-\frac{\nu_{31}}{E_{3}} & -\frac{\nu_{32}}{E_{3}} & \frac{1}{E_{3}} & 0 & 0 & 0 \\
0 & 0 & 0 & \frac{1}{G_{23}} & 0 & 0 \\
0 & 0 & 0 & 0 & \frac{1}{G_{13}} & 0 \\
0 & 0 & 0 & 0 & 0 & \frac{1}{G_{12}}
\end{array}\right]
$$

Para calculá-lo em coordenadas do material é necessário realizar a seguinte transformação 
$c_{i j k l}^{(l)}=\lambda_{i m} \lambda_{j n} \lambda_{k o} \lambda_{l p} c_{m n o p}$

para tensão plana, ${ }^{\sigma_{33}^{(l)}}=0$ e consequentemente ${ }^{T_{c}=0}$. Para deformação plana, na borda do furo

$$
\varepsilon_{33}^{(l)}=c_{3311}^{(l)} \sigma_{11}^{(l)}+c_{3333}^{(l)} \sigma_{33}^{(l)}=0
$$

E então o fator de restrição transversal pode ser escrito como

$$
T_{c}=\frac{\sigma_{33}^{(l)}}{\sigma_{11}^{(l)}}=-\frac{c_{3311}^{(l)}}{c_{3333}^{(l)}}
$$

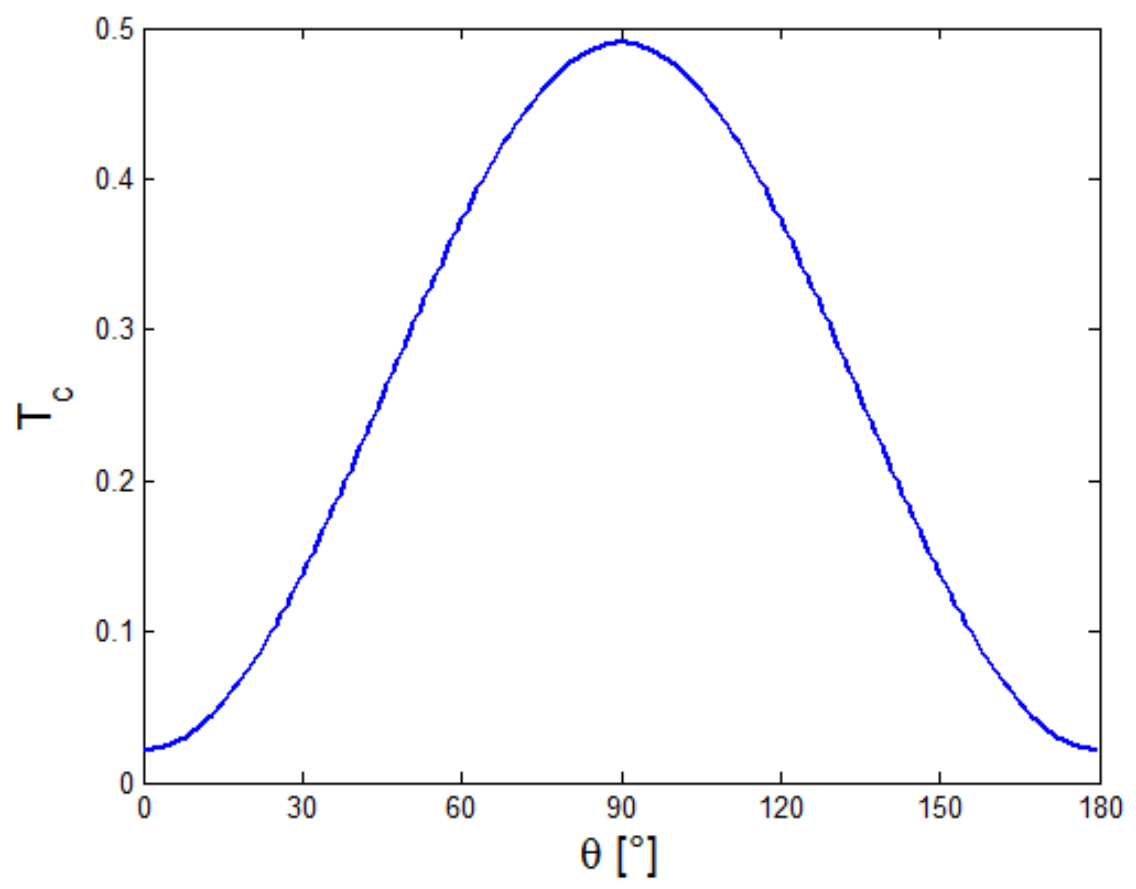

Figura 51. Variação do fator de restrição transversal na borda do furo para o caso de deformação plana

É importante perceber que $c_{3313}^{(l)}=0$ e $\sigma_{33}^{(l)}=\sigma_{33}$ ao longo da borda do furo porque o eixo de rotação é ao longo do plano $x_{1}-x_{2}$. Para um material isotrópico, $c_{3311}^{(l)}=c_{3311}$ e $c_{3333}^{(l)}=c_{3333}$, resultando em $T_{c}=\nu$, como observado por Góes et al. (2015). O fator de restrição varia ao longo da borda para um laminado unidirecional, já que as propriedades não são constantes, e utilizando as propriedades mostradas na Tabela 1, a variação do mesmo está mostrada na Figura 51. Como o fator de restrição não é constante, as componentes da tensão não são multiplicadas pelo mesmo valor ao longo da borda, 
resultando então em uma distribuição de tensões completamente diferentes das obtidas para o caso de tensão plana.

Considerando a hipótese de deformação plana como um limite extremo para placas espessas, os critérios de falha podem ser aplicados e para qualquer espessura é esperado que os resultados das resistências estejam entre a resistência para tensão plana e a resistência para deformação plana. Dessa maneira, o maior erro possível gerado por assumir a hipótese de tensão plana pode ser avaliado.

O formalismo de Stroh precisa sofrer algumas pequenas adaptações para o caso de deformação plana, mas os detalhes serão omitidos para evitar excessos na abordagem matemática e podem ser encontrados em Ting (1996) e Hwu (2009).

Para uma placa grande com um furo circular sob carregamento uniaxial, assim como considerado anteriormente para o caso de tensão plana, as componentes das tensões ao redor da borda do furo são mostradas na Figura 52. Note que agora tem uma componente a mais, $\sigma_{33}$, que pode ser até maior do que a tensão nominal, logo não é desprezível.

As resistências à tração e à compressão foram obtidas seguindo o mesmo procedimento mostrado anteriormente para a hipótese de tensão plana e estão mostradas na Figura 53. As resistências para tensão plana também são mostradas nesse gráfico para facilitar a comparação. Os resultados da resistência à tração continuam com uma tendência similar, mas para compressão as estimativas são bastante diferentes tanto na forma das curvas, quanto na magnitude das resistências. A maior variação notada é para LaRC05 porque o estado 3D de tensões influencia na instabilidade da fibra, como discutido por Pinho et al. (2006). 

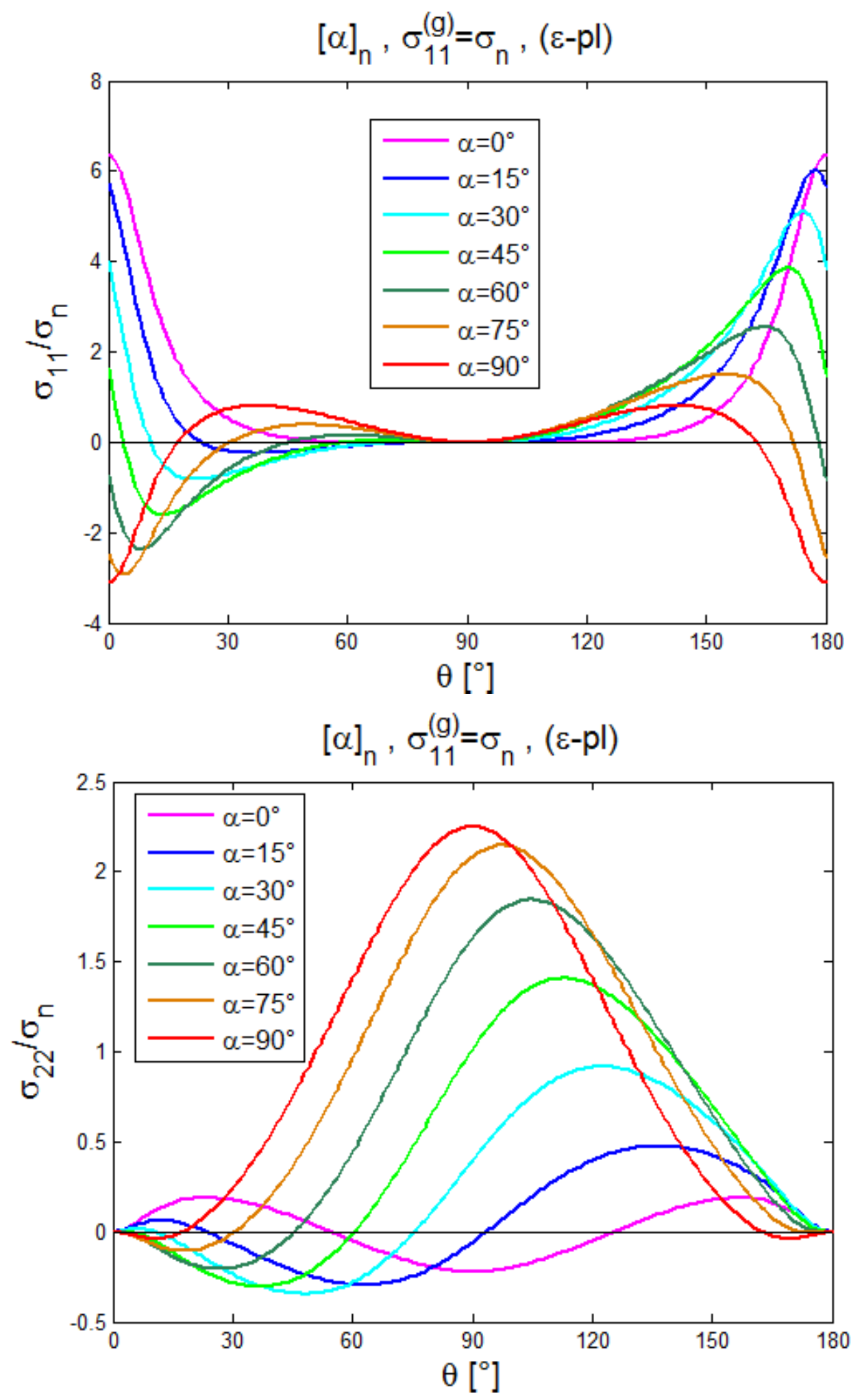

Figura 52 - Tensões ao longo da borda do furo em coordenadas do material para uma placa grande de laminado unidirecional com furo circular e carregada uniaxialmente considerando a hipótese de deformação plana (continua) 

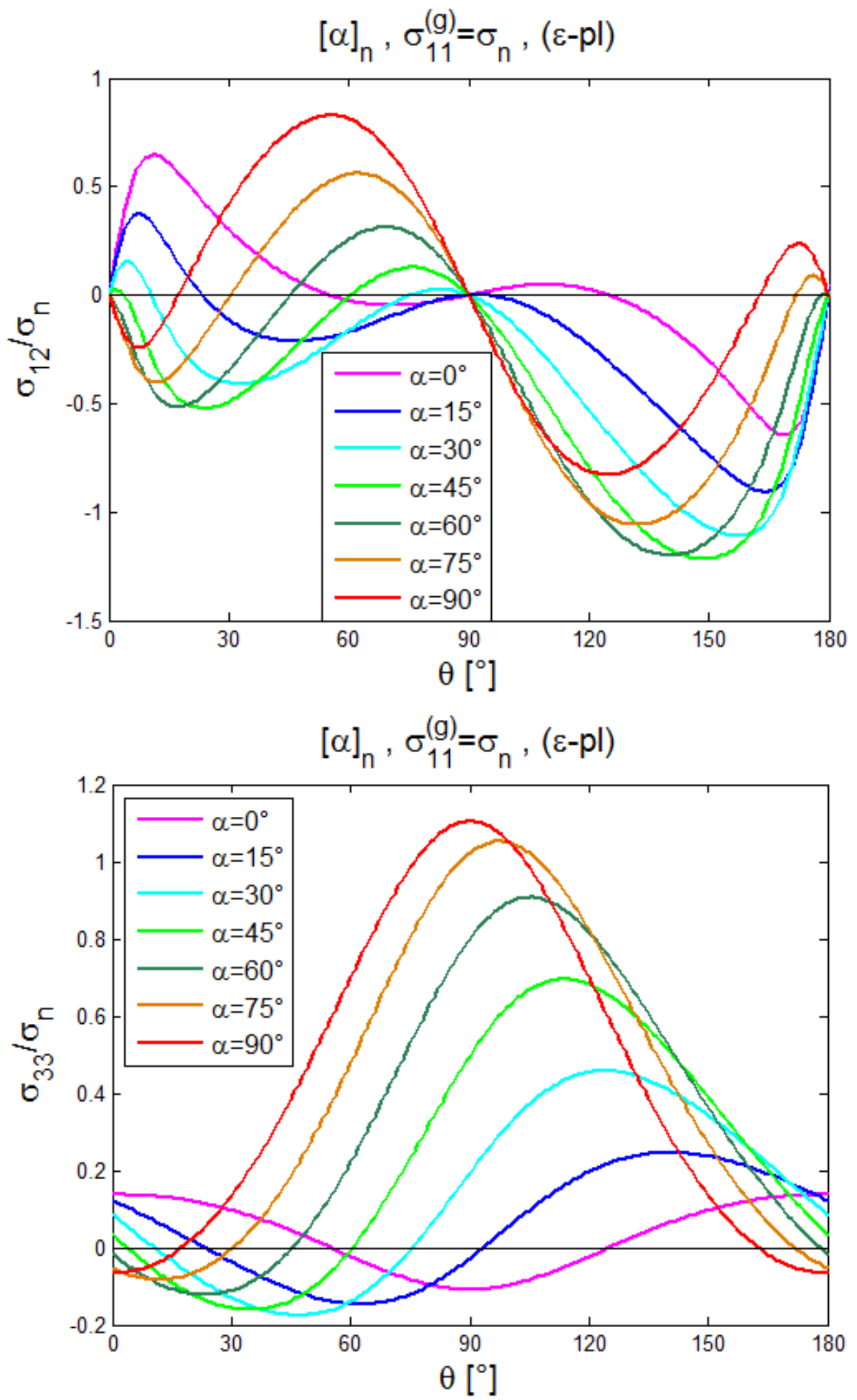

Figura 52 - Tensões ao longo da borda do furo em coordenadas do material para uma placa grande de laminado unidirecional com furo circular e carregada uniaxialmente considerando a hipótese de deformação plana (continuação) 

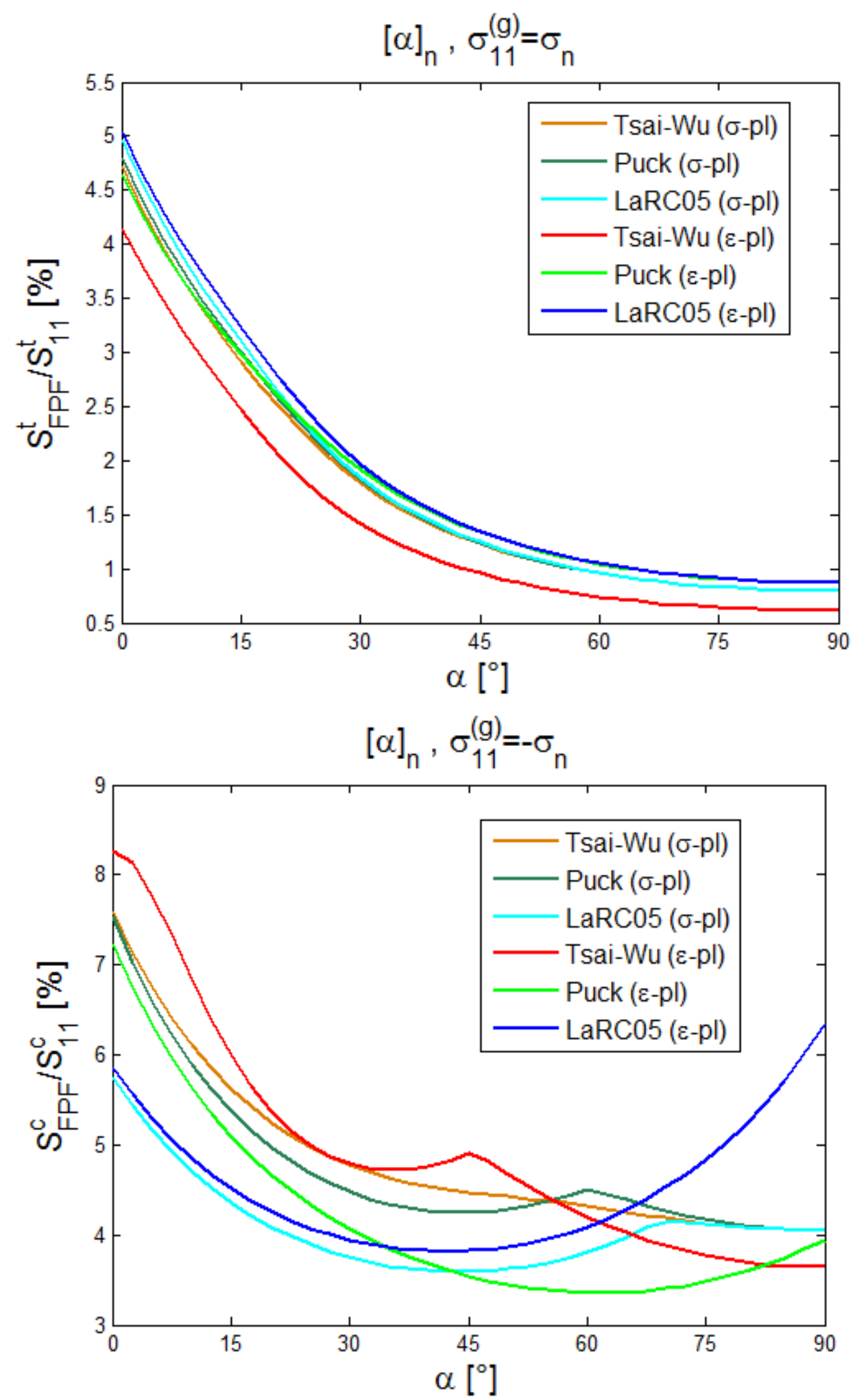

Figura 53 - Comparação entre as estimativas de $S_{F P F}^{t} / S_{11}^{t}$ e $S_{F P F}^{c} / S_{11}^{c}$ para placas grandes de laminados unidirecionais com furos circulares sob estado plano de tensões e estado plano de deformações (continuação) 

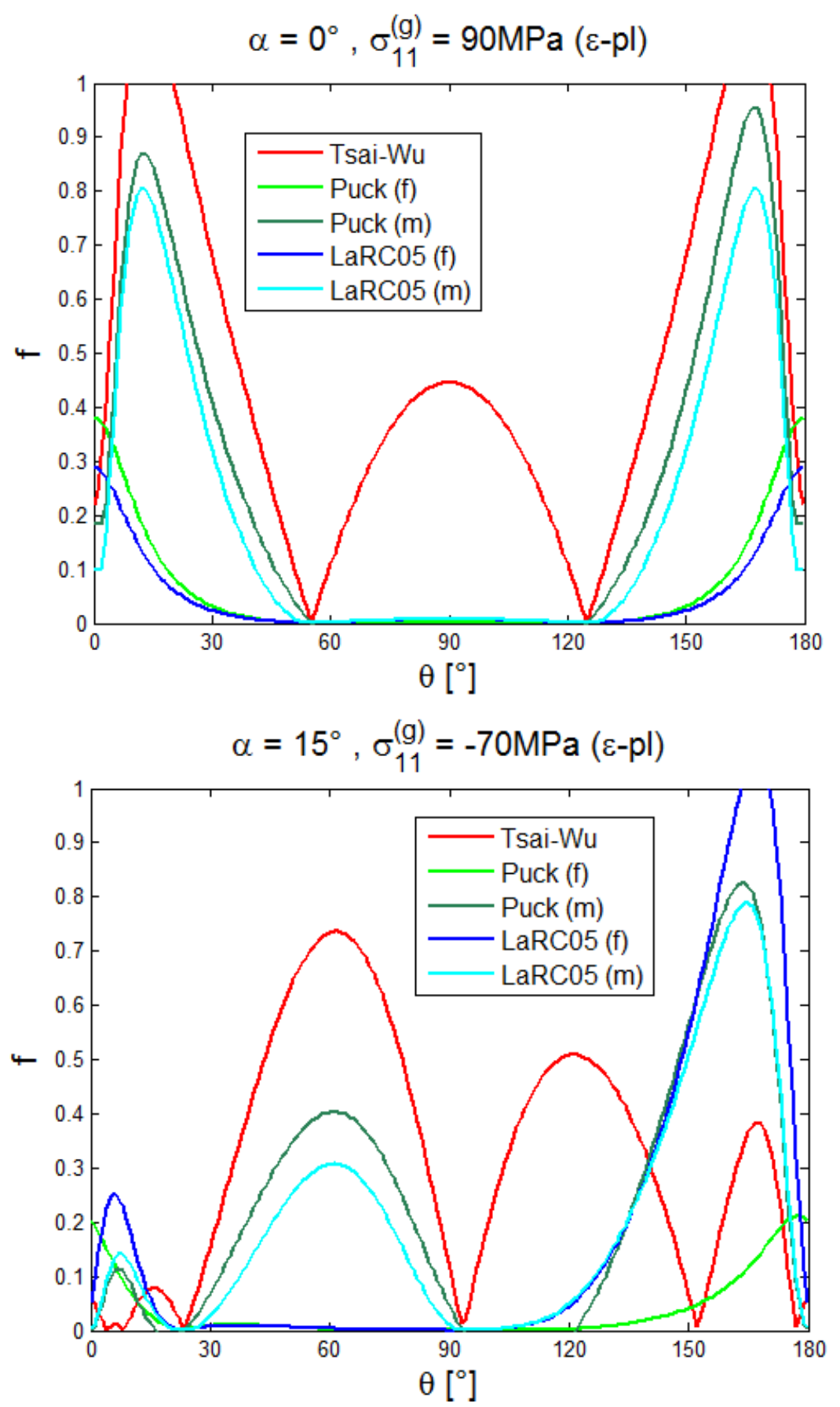

Figura 54 - Casos selecionados para carregamentos uniaxiais em uma placa grande com furo circular assumindo deformação plana (continua) 

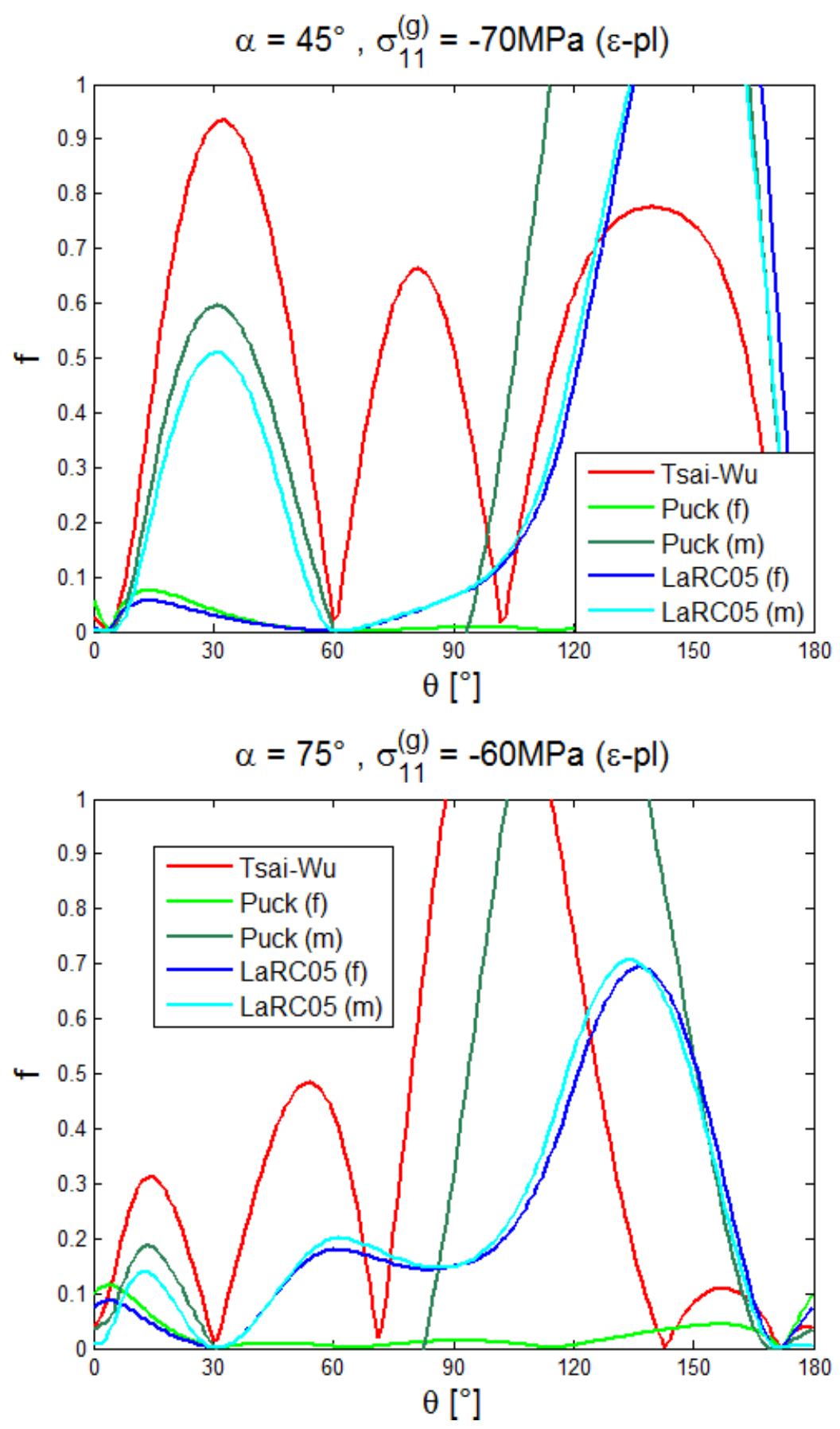

Figura 54 - Casos selecionados para carregamentos uniaxiais em uma placa grande com furo circular assumindo deformação plana (continuação)

Alguns casos foram selecionados para um estudo detalhado e estão mostrados na Figura 54. No primeiro, para $\sigma_{11}^{(g)}=90 M P a$ e $\alpha=0^{\circ}$, apenas Tsai-Wu indica falha devido à soma direta de uma componente a mais na função polinomial, enquanto os outros modelos 
avaliam o plano crítico que não é tão sensível a uma componente adicional de tensão normal. Para os carregamentos compressivos três diferentes resultados são mostrados: para $\sigma_{11}^{(g)}=-70 M P a$ e $\alpha=15^{\circ}$ apenas LaRC05 estima a falha (das fibras); para $\sigma_{11}^{(g)}=-70 M P a$ e $\alpha=45^{\circ}$, Tsai-Wu é o único modelo que indica uma condição segura de carregamento (sem falha); e para $\sigma_{11}^{(g)}=-60 M P a$ e $\alpha=75^{\circ}$ apenas LaRC05 não estima falha. Baseado nesses resultados, nenhuma conclusão definitiva sobre conservadorismo dos critérios pode ser apontada pela grande variação entre as estimativas, mas os resultados do WWFE-II (KADDOUR; HINTON, 2013) indicam que a busca pelo plano crítico consegue descrever adequadamente a falha para casos 3D, tornando os critérios com embasamento fenomenológicos mais recomendados (pelo menos para casos 3D de tensões).

Góes et al. (2014) e (2015) obtiveram uma diferença menor do que $10 \%$ na estimativa das resistências usando os critérios de Tresca de von Mises para materiais isotrópicos. Para o laminado estudado, as razões entre as resistências estimadas considerando as hipóteses de deformação plana e de tensão plana são mostradas na Figura 55. Considerar que a estrutura está sob tensão plana pode ser uma hipótese conservadora ou não, dependendo do critério de falha, do carregamento aplicado e do ângulo entre a direção de carregamento e das fibras.

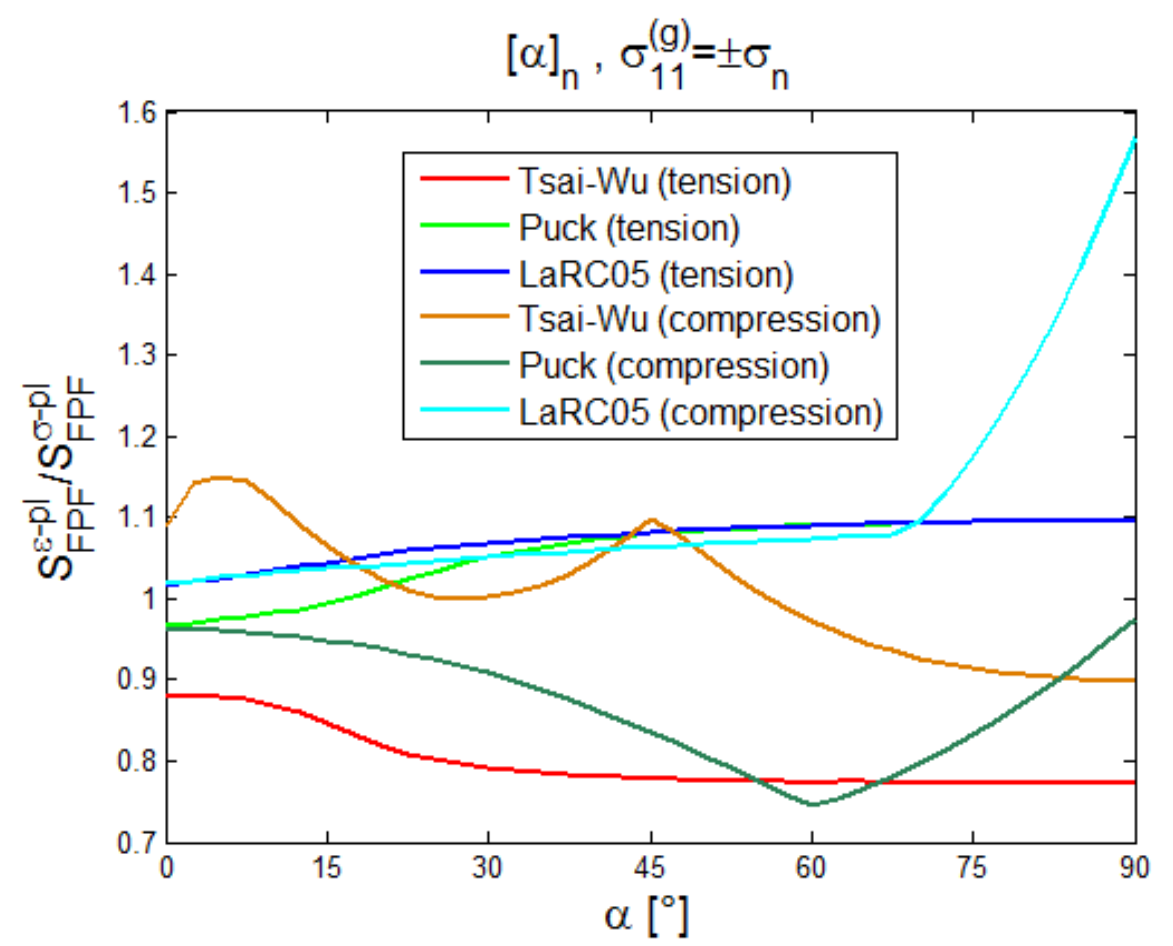

Figura 55 - Razão entre as resistências estimadas considerando deformação plana e tensão plana 
No entanto, alguns resultados alarmantes sobre a consideração da hipótese de tensão devem ser destacados: Tsai-Wu e Puck estimaram resistências que têm variações maiores do que 20\% quando as duas diferentes hipóteses 2D (tensão plana e deformação plana) são assumidas, o que é um resultado que ressalta a atenção que deve ser tomada; LaRC05 estimou diferença maior do que 50\% entre as resistências, reforçando ainda mais o cuidado que deve ser tomado durante as hipóteses do projeto e a necessidade de validação dos modelos. 


\section{Estudo de outros parâmetros afetam a concentração de tensão}

Nesse capítulo será feita uma breve abordagem sobre dois parâmetros que afetam a distribuição de tensão na borda do furo: o efeito das bordas em placas finitas e a fração volumétrica da quantidade de fibras na matriz. Para placas finitas, será mostrada uma aproximação popular na literatura para estimar a concentração de tensão. Para avaliar o efeito das fibras serão utilizados conceitos básicos da micromecânica para estimar as propriedades mecânicas e então a distribuição de tensão. Ambos os casos irão considerar um carregamento uniaxial em uma placa com furo circular.

\subsection{Estudo de placas finitas}

As soluções analíticas disponíveis na literatura consideram placas infinitas, como visto anteriormente. Apesar de essa aproximação ser bastante útil para muitos problemas práticos, em alguns casos se torna necessário considerar a influência de efeitos de borda na distribuição de tensões. Para tal, será utilizada uma abordagem numérica utilizando o Método dos Elementos Finitos (MEF). Apenas o carregamento uniaxial será considerado nessa seção.

Para materiais isotrópicos, a principal aproximação utilizada para obter o fator de concentração de tensão $\left(K_{t}\right)$ é (YOUNG; BUDYNAS, 2002)

$$
K_{t}=3.00-3.13\left(\frac{d}{H}\right)+3.66\left(\frac{d}{H}\right)^{2}-1.53\left(\frac{d}{H}\right)^{3}
$$

onde $d$ é o diâmetro do furo e $H$ é a largura da placa. Mesmo para materiais isotrópicos pode ser interessante obter toda a distribuição de tensão na borda do furo, pois mesmo a resistência sendo independente da direção em que sendo analisada, materiais frágeis podem ter resistência à tração diferente da resistência à compressão (CRANDALL et al., 1978).

Para materiais anisotrópicos a distribuição de tensão na borda do furo se torna ainda mais importante pela influência da direção no critério de falha como discutido anteriormente. Todavia, a aproximação mais popular na literatura é apenas para o $K_{t}$ e é mostrada abaixo (TAN; KIM, 1990). 


$$
\frac{K_{t}^{\infty}}{K_{t}}=\frac{3\left(1-\frac{d}{H}\right)}{2+\left(1-\frac{d}{H}\right)^{3}}+\frac{1}{2}\left(\frac{d}{H} M_{K_{t}}\right)^{6}\left(K_{t}^{\infty}-3\right)\left(1-\left(\frac{d}{H} M_{K_{t}}\right)^{2}\right)
$$

onde $K_{t}^{\infty}$ é o $K_{t}$ para o placa infinita e

$$
M_{K_{t}}^{2}=\frac{\sqrt{1-8\left(\frac{3\left(1-\frac{d}{H}\right)}{2+\left(1-\frac{d}{H}\right)^{3}}-1\right)}-1}{2\left(\frac{d}{H}\right)^{2}}
$$

Supondo que a aproximação mostrada na Equação (164) possa ser estendida para se obter toda a distribuição de tensão, tem-se

$$
\frac{\left.\sigma_{11}^{(l)}\right|^{\infty}}{\sigma_{11}^{(l)}}=\frac{3\left(1-\frac{d}{H}\right)}{2+\left(1-\frac{d}{H}\right)^{3}}+\frac{1}{2}\left(\frac{d}{H} M\right)^{6}\left(\left.\sigma_{11}^{(l)}\right|^{\infty}-3\right)\left(1-\left(\frac{d}{H} M\right)^{2}\right)
$$

onde $\left.\sigma_{11}^{(l)}\right|^{\infty}$ será utilizado para representar a tensão para a placa infinita definida anteriormente na seção.

Para comparar as estimativas do modelo de Tan será utilizada uma simulação computacional através do MEF. Considerando as geometrias 2D e assumindo o estado plano de tensões, o elemento PLANE183 foi utilizado pela capacidade quadrática de obter a solução que se torna útil para descrever geometrias irregulares com concentradores de tensões. O elemento PLANE183 pode ter 8 ou 6 nós, dependendo se o mesmo for retangular ou triangular.

Para obter uma melhor qualidade da malha, a placa foi subdividida em sete regiões mas com três principais formas diferentes (ver Figura 56): um anel concêntrico ao furo (1); quatro partes iguais que dividem um quadrado (2); e as duas partes externas a essas subdivisões que formam a geometria da placa (3). 


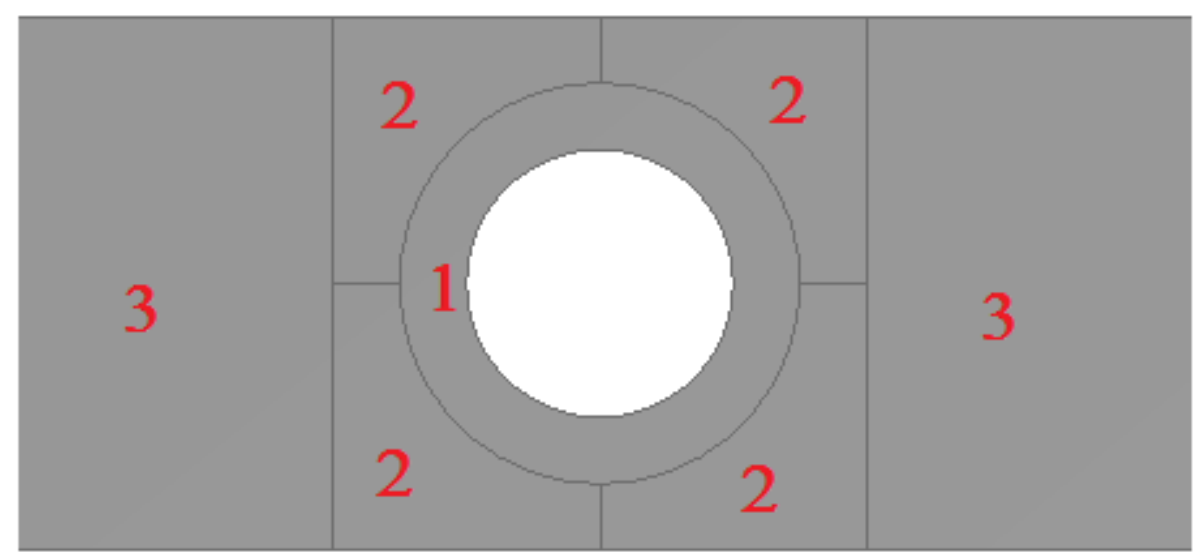

Figura 56 - Regiões utilizadas para parametrizar a malha

Três principais parâmetros foram utilizados para definir as diferentes malhas: o número de divisões tangenciais na borda do furo; o número de divisões radiais do anel; e número de divisões retangulares na região 2. A Tabela 4 mostra o resumo dos parâmetros para as malhas convergentes e a Figura 57 mostra um exemplo para $d / H=0.5$. A malha foi considerada convergente considerando um erro menor do que $1 \%$.

Tabela 4 - Parâmetros das malhas convergentes

\begin{tabular}{c|c|c|c}
\hline $\boldsymbol{d} / \boldsymbol{H}$ & 0.1 & 0.3 & 0.5 \\
\hline Div. Tangenciais & 240 & 240 & 240 \\
\hline Div. Radiais & 10 & 10 & 10 \\
\hline Div. Retangulares & 50 & 15 & 10 \\
\hline Nós & 51086 & 26796 & 23890 \\
\hline Elementos & 16834 & 8682 & 7678 \\
\hline
\end{tabular}



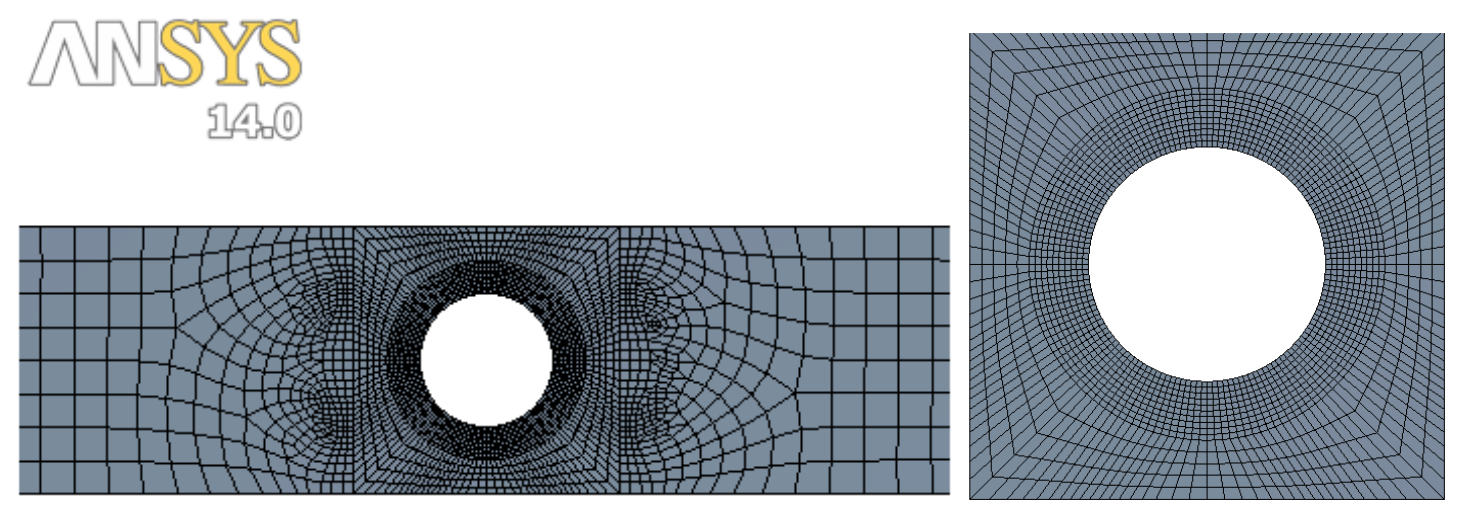

Figura 57 - Exemplo de malha utilizada para $d / H=0.5$

Para evitar informações excessivas, apenas um exemplo do erro gerado pela utilização dessa aproximação está mostrado na Figura 58. Note que o erro pode ser maior do que $30 \%$, mostrando que essa aproximação não gera resultados aceitáveis na maioria dos casos.

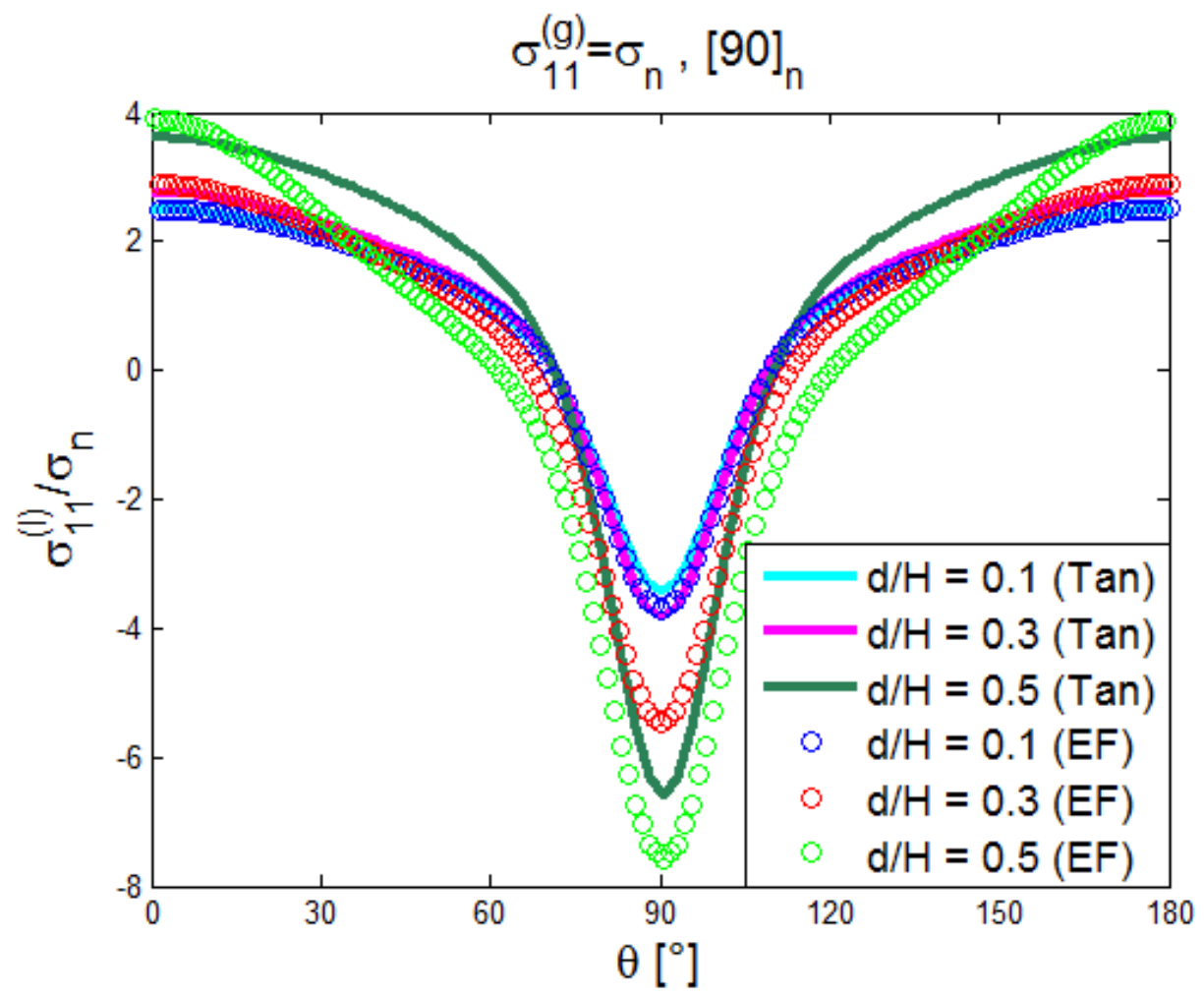

Figura 58 - Comparação entre as estimativas para placas finitas utilizando o modelo proposto por Tan e as soluções utilizando o MEF 
Dessa forma, conclui-se que não há nenhuma aproximação razoável na literatura para estimar a tensão na borda de furo para placas ortotrópicas finitas (pelo menos do conhecimento do autor deste trabalho), sendo os métodos numéricos indispensáveis.

\subsection{Uma breve abordagem sobre o efeito das fibras na concentração de tensão}

A anisotropia de um laminado é resultado da adição de fibras em uma matriz isotrópica, resultando assim em propriedades diferentes de acordo com a direção. Dessa forma, serão introduzidos conceitos básicos de micromecânica nesse capítulo para estimar as propriedades mecânicas equivalentes de acordo com a fração volumétrica de fibra e então avaliar o seu efeito na distribuição de tensão.

A abordagem mais tradicional de se estimar as propriedades mecânicas de uma lâmina é conhecida como a Regra das Misturas (JONES, 1998) que considera que as fibras e a matriz se comportam de forma semelhante a molas em série e em paralelo, de acordo com o carregamento, e pode-se então obter as estimativas das propriedades mecânicas equivalentes como

$$
\begin{aligned}
& E_{1}=E^{(f)} V^{(f)}+E^{(m)} V^{(m)} \\
& E_{2}=\frac{E^{(f)} E^{(m)}}{E^{(f)} V^{(f)}+E^{(m)} V^{(m)}} \\
& v_{12}=v^{(f)} V^{(f)}+V^{(m)} V^{(m)} \\
& G_{12}=\frac{G^{(f)} G^{(m)}}{G^{(f)} V^{(f)}+G^{(m)} V^{(m)}}
\end{aligned}
$$

Apesar de essas equações serem baseadas em hipóteses simplistas, são de grande valia para um estudo inicial e o entendimento físico do problema. Muitas outras abordagens podem ser encontradas na literatura baseadas em métodos de elasticidade, princípios variacionais, representação da célula de simetria por elementos finitos e teoria da homogeneização (CHRISTENSEN, 2005 e YOUNES et al., 2012).

Halpin e Tsai (1969) ressaltaram o fato de que a regra das misturas não proporciona resultados aceitáveis para estimar $E_{2}$ e $v_{12}$ e que os métodos baseados na teoria da 
elasticidade resultavam em equações muito trabalhosas, propondo então o uso das seguintes equações:

$$
\begin{aligned}
& E_{1}=E^{(f)} V^{(f)}+E^{(m)}\left(1-V^{(f)}\right) \\
& E_{2}=\frac{1+\xi_{E_{2}} \eta_{E_{2}} V^{(f)}}{1-\eta_{E_{2}} V^{(f)}} E^{(m)} \\
& v_{12}=v^{(f)} V^{(f)}+v^{(m)}\left(1-V^{(f)}\right) \\
& G_{12}=\frac{1+\xi_{G_{12}} \eta_{G_{12}} V^{(f)}}{1-\eta_{G_{12}} V^{(f)}} G^{(m)}
\end{aligned}
$$

onde

$$
\begin{aligned}
& \eta_{E_{2}}=\frac{E^{(f)}-E^{(m)}}{E^{(f)}+\xi_{E_{2}} E^{(m)}} \\
& \eta_{G_{12}}=\frac{G^{(f)}-G^{(m)}}{G^{(f)}+\xi_{G_{12}} G^{(m)}}
\end{aligned}
$$

sendo $\xi_{E_{2}}$ e $\xi_{G_{12}}$ parâmetros que podem ser calibrados experimentalmente. Halpin e Kardos (1976) fizeram um estudo detalhado sobre a base teórica do modelo apresentado anteriormente e mostraram que na ausência de dados experimentais pode-se estimar como função da fração volumétrica

$$
\begin{aligned}
& \xi_{E_{2}}=2+40\left(V^{(f)}\right)^{10} \\
& \xi_{G_{12}}=1+40\left(V^{(f)}\right)^{10}
\end{aligned}
$$

Entretanto, para fibras transversalmente isotrópicas ao invés de isotrópicas, as seguintes alterações serão feitas

$$
\begin{aligned}
& E_{1}=E_{1}^{(f)} V^{(f)}+E^{(m)}\left(1-V^{(f)}\right) \\
& v_{12}=v_{12}^{(f)} V^{(f)}+v^{(m)}\left(1-V^{(f)}\right) \\
& \eta_{E_{2}}=\frac{E_{2}^{(f)}-E^{(m)}}{E_{2}^{(f)}+\xi_{E_{2}} E^{(m)}}
\end{aligned}
$$




$$
\eta_{G_{12}}=\frac{G_{12}^{(f)}-G^{(m)}}{G_{12}^{(f)}+\xi_{G_{12}} G^{(m)}}
$$

Considerando que as propriedades mecânicas são conhecidas para a lâmina com $V_{f}=0.6$ (ver Tabela 1), pode-se ajustar as Equações (169.a,b) da seguinte forma

$$
\begin{aligned}
& \xi_{E_{2}}=\xi_{E_{2}}^{0}+40\left(V^{(f)}\right)^{10} \\
& \xi_{G_{12}}=\xi_{G_{12}}^{0}+40\left(V^{(f)}\right)^{10}
\end{aligned}
$$

E então, manipulando algebricamente, obtêm-se

$$
\begin{aligned}
& \xi_{E_{2}}^{0}=\frac{\left(E_{2}^{(f)} E^{(m)}-E_{2}^{(f)} E_{2}^{\left(V^{(f)}=0.6\right)}+\left(E_{2}^{(f)}-E^{(m)}\right) E_{2}^{\left(V^{(f)}=0.6\right)} V^{(f)}\right)}{E^{(m)}\left(E_{2}^{\left(V^{(f)}=0.6\right)}-E^{(m)}-\left(E_{2}^{(f)}-E^{(m)}\right) V^{(f)}\right)}-40\left(V^{(f)}\right)^{10} \\
& \xi_{G_{12}}^{0}=\frac{\left(G_{12}^{(f)} G^{(m)}-G_{12}^{(f)} G_{12}^{\left(V^{(f)}=0.6\right)}+\left(G_{12}^{(f)}-G^{(m)}\right) G_{12}^{\left(V^{(f)}=0.6\right)} V^{(f)}\right)}{G^{(m)}\left(G_{12}^{\left(V^{(f)}=0.6\right)}-G^{(m)}-\left(G_{12}^{(f)}-G^{(m)}\right) V^{(f)}\right)}-40\left(V^{(f)}\right)^{10}
\end{aligned}
$$

Dessa forma, como o objetivo do presente trabalho não é a abordagem micromecânica, apenas essa breve introdução será mostrada e as Equações (167-172) serão utilizadas para estimar as propriedades mecânicas elásticas.

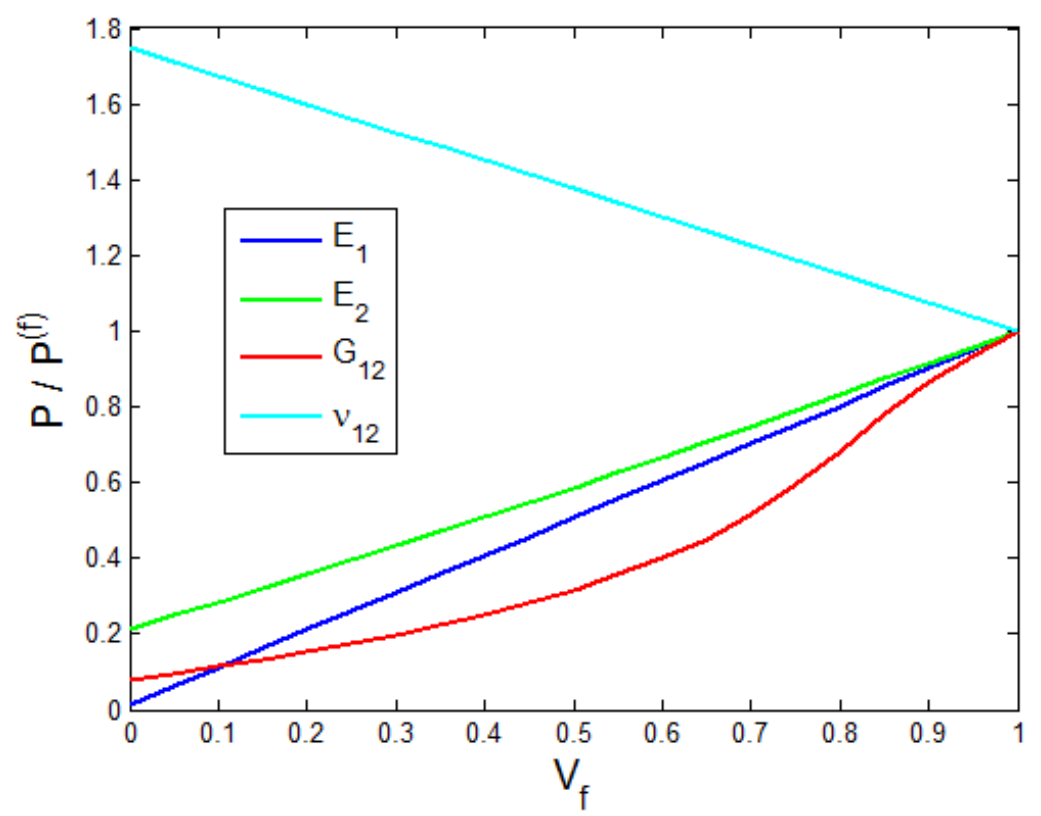

Figura 59 - Variação das propriedades mecânicas da lâmina de acordo com a fração volumétrica de fibras 
As resistências não serão estimadas, uma vez que as aproximações mostradas não reproduzem satisfatoriamente os resultados experimentais para as mesmas, sendo então o presente estudo restrito à análise de distribuição de tensões. Para as propriedades das fibras e da matriz mostras na Tabela 1, as propriedades mecânicas da lâmina de acordo com a fração volumétrica são mostradas na Figura 59, onde as mesmas são normalizadas em relação às propriedades da fibra.

Uma vez que as propriedade são obtidas, pode-se aplicar as equações desenvolvidas anteriormente para calcular a concentração de tensão atuando na borda do furo. Para um carregamento uniaxial, as distribuições de tensões considerando $V_{f}=0.0,0.3,0.5,0.8$ são mostradas na Figura 60 para fibras com inclinações $\alpha=0^{\circ}, 45^{\circ}, 90^{\circ}$. Pode-se, então, concluir que a fração volumétrica das fibras não altera de forma significativa a distribuição de tensões, mas espera-se que as resistências tenham alterações consideráveis.

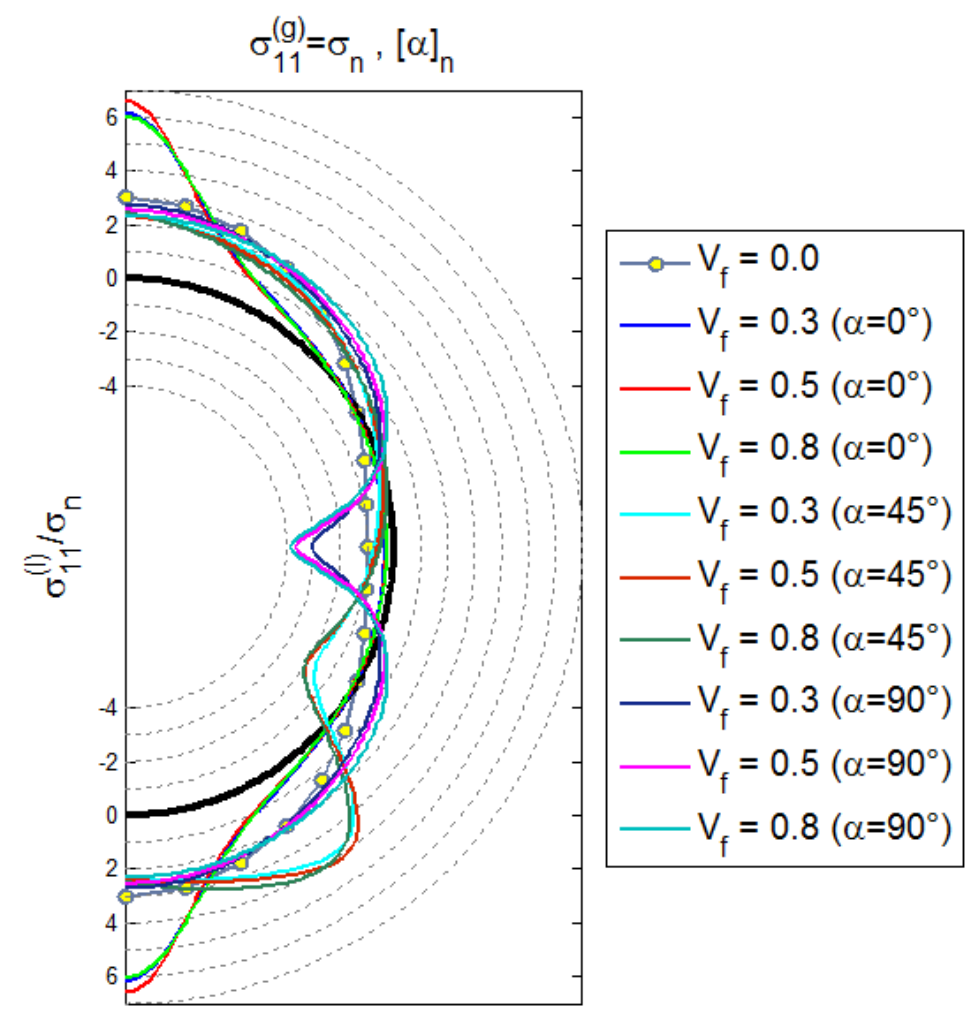

Figura 60 - Influência da fração volumétrica de fibras na concentração de tensão ao longo da borda do furo 


\section{Conclusões}

A utilização de entalhes é necessária, apesar de diminuir a resistência mecânica, em todas as aplicações práticas por requisitos de funcionamento do equipamento. Esses entalhes, por resultarem em mudança brusca na geometria, resultam em perturbações nos campos de tensões que geram efeitos concentrações de tensões.

O objetivo desse trabalho é estudar efeitos de concentrações de tensões em materiais anisotrópicos, particularmente compósitos laminados. Inicialmente foi mostrada a base teórica necessária para o estudo analítico de forma mais breve possível, mas sem ser simplista no rigor matemático necessário. O formalismo de Stroh e a TCL foram as ferramentas apresentadas e utilizadas no desenvolvimento do trabalho para a abordagem analítica que, apesar das facilidades computacionais atuais, é sempre de fundamental importância para o entendimento físico.

Uma vez que a distribuição de tensões pode ser obtida, torna-se necessário estudar os critérios de falha. Os critérios de Tsai-Wu, Puck e LaRC05 foram detalhadamente estudados, considerando sempre a falha como início do dano (mecanismos de progressão do dano não foram estudados). Os mesmos foram escolhidos com base nos resultados e recomendações do WWFE.

Tendo, então, toda a base teórica necessária para o estudo do efeito de concentradores de tensões em laminados, mais especificamente furos circulares e elípticos, alguns casos de interesse práticos foram estudados, sempre com o objetivo de comparar os resultados da metodologia apresentada com os resultados conhecidos para materiais isotrópicos.

Mesmo para o laminado mais simples, que é o unidirecional, com furo circular e apenas com carregamento uniaxial, foi mostrado que a concentração de tensão pode chegar a aproximadamente sete vezes a tensão nominal enquanto para o material isotrópico esse valor é igual a três. $\mathrm{O}$ efeito na resistência do laminado unidirecional tem um resultado ainda mais alarmante, a resistência ao início do dano pode diminuir até vinte vezes.

A diferença entre o carregamento de cisalhamento puro e o carregamento biaxial de tração/compressão (que para materiais isotrópicos são equivalentes) para materiais anisotrópicos foi mostrada. Os mesmo não devem ser diretamente relacionados por causa 
da importância do ângulo entre a direção de carregamento e as fibras (vale ressaltar mais uma vez que o principio da superposição continua sendo válido uma vez que o material está em regime linear elástico, mas deve ser aplicado com cuidado por causa da importância da direção das propriedades do material).

Apesar de entalhes não serem desejados do ponto de vista estrutural, são inevitáveis em muitos casos. Supondo que exista o entalhe e a inclinação das fibras seja a variável, foi mostrado que diminuir a concentração de tensão não deve ser uma condição de projeto. Pelo contrário, em alguns casos ter a concentração de tensão máxima é equivalente a maximizar a resistência porque a tensão máxima está atuando nas fibras.

Foi visto também que laminados do tipo angle-ply tendem a ter uma resistência ao início do dano menor do que para o laminado unidirecional equivalente se a placa tiver furos. O que não é um resultado esperado uma vez que o contrário acontece para placas sem entalhes.

A hipótese de tensão plana foi posta à prova comparando os resultados da mesma para os resultados de casos de placas similares com carregamentos iguais, mas considerando a hipótese de deformação plana. Dessa forma, o efeito da espessura pode ser avaliado de forma simples e eficiente pelas duas hipóteses extremas, sendo esperado que qualquer espessura resulte em valores entre esses dois limites. A diferença entre as estimativas chegou a ser maior do que $20 \%$ para os critérios de Tsai-Wu e Puck e maior do que $50 \%$ para o LaRC05.

Os critérios de falha tenderam a apresentar estimativas próximas na maioria dos casos, sendo a maioria das falhas estimadas da matriz. A maior diferença verificada foi na modelagem da falha da fibra sob compressão pelo modelo LaRC05, que resultou em estimativas de resistência consideravelmente menores. Sendo, então, uma sugestão para possíveis trabalhos futuros, a realização de testes experimentais para verificar essas estimativas.

Por último, mostrou-se que as aproximações existentes na literatura para estimar o efeito de placas finitas não alcançam resultados satisfatórios, sendo a modelagem numérica a melhor abordagem para estudar o efeito de concentrações de tensões em placas onde a dimensão do furo não seja muito menor do que as dimensões da placa. Viu-se também que 
a fração volumétrica das fibras não altera de forma significativa a concentração de tensão, todavia espera-se a resistência seja bastante influenciada.

Como sugestão para trabalhos futuros, vale mencionar a necessidade do desenvolvimento de testes experimentais para validação dos resultados previstos e o estudo da propagação do dano até a ruptura total da peça.

Resumidamente, foram mostrados aspectos teóricos da modelagem de furos em placas compósitas de laminados e mostrou-se que os mesmos podem ser bem mais danosos do que os equivalentes em placas isotrópicas. Por último, vale lembrar que toda a distribuição de tensão ao longo da borda do furo é necessária para estimar a falha; o valor pontual nunca deve ser utilizado. 


\section{Referências bibliográficas}

AKBULUT, M.; XONMEZ, F. O. Optimum design of composite laminates for minimum thickness. Computers and Structures, 86, 1974-1982, 2008.

BARNETT, D. M.; LOTHE, J. Synthesis of the Sextic and the Integral Formalism for Dislocation, Green's Function and Surface Waves in Anisotropic Elastic Solids, Phys. Norv., Vol. 7, p. 13-19, 1973.

BERBINAU, P.; FILIOU, C.; SOUTIS, C. Stress and Failure Analysis of Composite Laminates with an Inclusion under Multiaxial Compression-Tension Loading, Applied Composite Materials, 8, 307-326, 2001.

CAStRO, J. T. P.; MEGGiOlaro, M. A. Fatigue Design Techniques: Vol. I - HighCycle Fatigue, CreateSpace Independent Publishing Platform, 2016.

CAStRO, J. T. P.; MEGGIOLARO, M. A. Fatigue Design Techniques: Vol. II - LowCycle and Multiaxial Fatigue, CreateSpace Independent Publishing Platform, 2016.

CHAWLA, K. K. Composite Materials, Science and Engineering, Third Edition, Springer, 2012.

CHEN, B.Y.; Tay, T.E.; Baiz, P.M.; Pinho, S.T. Numerical Analysis of Size Effects on Open-Hole Tensile Composite Laminates, Composites, 47, Part A, 52-62, 2013.

CHRISTENSEN, R. M. Mechanics of Composite Materials, Second Edition, New York, Dover, 2005.

CRANDALL, S. H.; DAHL, N. C.; LARDNER, T. J. An Introduction to the Mechanics of Solids, 2. Ed., Singapore, McGraw-Hill, 1978.

CUNTZE, R. G.; FREUND, A. The Predictive Capability of Failure Mode Concept-Based Strength Criteria for Multidirectional Laminates, Composites Science and Technology, V. 64, I. 3-4, p. 343-377, Mar. 2004.

DANIEL, I. M.; ISHAI, O. Engineering Mechanics of Composite Materials, New York, Oxford University Press, 1994.

DÁVILA, C. G.; CAMANHO, P. P. Failure Criteria for FRP Laminates in Plane Stress, NASA/TM-2003-212663, 2003.

DÁVILA, C. G.; CAMANHO, P. P.; ROSE, C. A. Failure Criteria for FRP Laminates, Journal of Composite Materials, N. 4, Vol. 39, p. 323-345, Feb. 2005. 
DEUSCHLE, H. M.; PUCK, A. Application of the Puck Failure Theory for FibreReinforced Composites Under Three-Dimensional Stress: Comparison with Experimental Results, Journal of Composite Materials, 47(6-7), 827-846, 2013.

GHIASI, H.; FAYAZBAKHSH, K.; PASINI, D.; LESSARD, L. Optimum Stacking Sequence Design of Composite Materials: Part II: Variable Stiffness Design, Composite Structures, Vol. 93, I. 1, p. 1-13, Dec. 2010.

GHIASI, H.; PASINI, D.; LESSARD, L. Optimum Stacking Sequence Design of Composite Materials: Part I: Constant Stiffness Design, Composite Structures, 90, p. 1$11,2009$.

GÓES, R. C. O.; CASTRO, J. T. P.; MARTHA, L. F. 3D Effects Around Notch and Crack Tips, International Journal of Fatigue, 62, p. 159-170, 2014.

GÓES, R. C. O.; CASTRO, J. T. P.; MEGGIOLARO, M. A. 3D Thickness Effects Around Notch and Crack Tip Stress/Strain Fields, Frattura ed Integrità Strutturale, 33, p. 89-96, 2015.

GREEN, A. E.; ZERNA, W. Theoretical Elasticity. 2. ed. New York, Dover Publications, 1968.

GREEN, B. G.; WISNOM, M. R.; HALLETT, S. R. An Experimental Investigation into the Tensile Strength Scaling of Notched Composites, Composites: Part A, 38, p. 867-878, 2007.

GRIFFITH, A. A. The Phenomena of Rupture and Flow in Solids, Phil. Trans. Roy. Soc. of London A, vol. 221, p. 163-198, 1921.

HALLETT, S. R.; GREEN, B.G.; JIANG, W.G.; WISNOM, M.R. An Experimental and Numerical Investigation into the Damage Mechanisms in Notched Composites,

Composites: Part A, 40, p. 613-624, 2009.

HALPIN, J. C.; KARDOS, J. L. The Halpin-Tsai Equations: A Review, Polymer Engineering and Science, Vol. 16, No. 5, May 1976.

HALPIN, J. C.; TSAI, S. W. Effects of Environmental Factors on Composite Materials. AFML-TR-67-423, 1969.

HINTON, M. J.; KADDOUR, A. S.; SODEN, P. D. (Orgs). Failure Criteria in Fibre Reinforced Polymer Composites: The World-Wide Failure Exercise, Elsevier, 2004.

HUANG, Y.; HA, S. K.; KOYANAGI, J.; MELO, J. D. D.; KUMAZAWA, H.; SUSUKI, I. Effects of an Open Hole on the Biaxial Strengths of Composite Laminates, Journal of Composite Materials, 44: 24-29, 2010. 
HWU, C. Anisotropic Plates with Various Openings under Uniform Loading or Pure Bending, J. Appl. Mech., 57(3), 700-706, Sep. 1990.

HWU, C. Anisotropic Elastic Plates. New York, Springer. 2009.

HWU, C. Polygonal Holes in Anisotropic Media, International Journal of Solids and Structures, Vol. 29, I. 19, p. 2369-2384, 1992.

HWU, C.; TING, T. C. T. Two-Dimensional Problems of the Anisotropic Elastic Solid with an Elliptic Inclusion, Q J Mechanics Appl Math, 42 (4): 553-572, 1989.

IARVE, E. V.; MOLLENHAUER, D.; KIM, R. Theoretical and Experimental Investigation of Stress Redistribution in Open Hole Composite Laminates Due to Damage Accumulation, Composites: Part A, 36, p. 163-171, 2005.

INGLIS, C. E. Stresses in a Plate Due to the Presence of Cracks and Sharp Corners, Trans. Inst. Naval Architects, 55, p. 219-241, 1913.

JING, Z.; FAN, X.; SUN, Q. Stacking Sequence Optimization of Composite Laminates for Maximum Buckling Load Using Permutation Search Algorithm, Composite Structures, 121, 225-236, 2015.

JONES, R. M. Mechanics of Composite Materials, 2. Ed., Taylor \& Francis Editions, 1998.

KADDOUR, A. S.; HINTON, M. J.; Input Data For Test Cases Used In Benchmarking Triaxial Failure Theories of Composites, Journal of Composite Materials, 46(19-20) 2295-2312, 2012.

KADDOUR, A. S.; HINTON, M. J. Maturity of 3D Failure Criteria for Fibre Reinforced Composites: Comparison Between Theories and Experiments: Part B of WWFE-II, Journal of Composite Materials, 47(6-7), 925-966, 2013.

KADDOUR, A. S.; HINTON, M. J.; SMITH, P. A.; LI, S. The Background to the Third World-Wide Failure Exercise, Journal of Composite Materials, 47: 2417-2426, 2013.

KALITA, K.; SHINDE, D.; THOMAS, T. T. Non-Dimensional Stress Analysis of an Orthotropic Plate, Materials Today: Proceedings, 2, 3527-3533, 2015.

Kaman, M. O. Effect of Fiber Orientation on Fracture Toughness of Laminated Composite Plates [0/0]s, Engineering Fracture Mechanics, 78, 2521-2534, 2011.

KANT, T.; SWAMINATHAN, K. Estimation of Transverse/Interlaminar Stresses in Laminated Composites: A Selective Review and Survey of Current Developments, Composite Structures, 49, 65-75, 2000. 
KHECHAI, A.; TATI, A.; GUETTALA, A. Finite Element Analysis of Stress

Concentrations and Failure Criteria in Composite Plates with Circular Holes, Front. Mech.

Eng., 9(3): 281-294, 2014.

KNOPS, M. Analysis of Failure in Fiber Polymer Laminates: The Theory of Alfred Puck. Leipzig, Springer, 2008.

KONISH, H. J.; WHITNEY, J. M. Approximate Stresses in an Orthotropic Plate Containing a Circular Hole, J. Comp. Mater., 9, 157-166, 1975.

KUREEMUN, U.; RIDHA, M.; TAY, T. E. Biaxial Tensile-Compressive Loading of Unnotched and Open-Hole Carbon Epoxy Crossply Laminates, Journal of Composite Materials, Vol. 49(23), 2817-2837, 2015.

LEE, J.; SOUTIS, C. Measuring the Notched Compressive Strength of Composite Laminates: Specimen Size Effects, Composites Science and Technology, 68, 2359-2366, 2008.

LEKHNITSKII, S. G. Anisotropic Plates. 3. Ed., New York, Gordon and Breach Science Publishers, 1987.

LEKHNITSKII, S. G. Theory of Elasticity of an Anisotropic Body, Moscow, Mir, 1981.

LIU, K. S.; TSAI, S.W. A Progressive Quadratic Failure Criterion for a

Laminate, Composites Science and Technology, Vol. 58, I. 7, p. 1023-1032, Jul. 1998.

MEGGIOLARO, M. A; MIRANDA, A. C. O.; CASTRO, J. T. Short Crack Threshold Estimates to Predict Notch Sensitivity Factors in Fatigue, International Journal of Fatigue, 29, 2022-2031, 2007.

MOLLENHAUER, D.; IARVE, E. V.; KIM, R.; LANGLEY, B. Examination of Ply Cracking in Composite Laminates with Open Holes: A Moiré Interferometric and Numerical Study. Composites: Part A, 37, p. 282-294, 2006.

MORAIS, A. B. Open-Hole Tensile Strength of Quasi-Isotropic Laminates, Composites Science and Technology, 60, 1997-2004, 2000.

MOURE, M. M.; OTERO, F.; GARCÍA-CASTILLO , S.K.; SÁNCHEZ-SÁEZ, S.; BARBERO, E.; BARBERO, E.J., Damage Evolution in Open-Hole Laminated Composite Plates Subjected to In-Plane Loads, Composite Structures, 133, 1048-1057, 2015.

MUSKHELISHVILI, N. I. Some Basic Problems of the Mathematical Theory of Elasticity. Moscow, Academy of Sciences of USSR, 1954.

NIKISHKOV, Y.; SEON, G.; MAKEEV, A. In-situ measurements of structural damage in notched composite laminates. In: Proceedings to $20^{\text {th }}$ INTERNATIONAL CONFERENCE ON COMPOSITE MATERIALS, ICCM 2015, Copenhagen, 2015. 
O’HIGGINS, R. M.; McCARTHY, M. A.; McCARTHY, C. T. Comparison of Open Hole Tension Characteristics of High Strength Glass and Carbon Fibre-Reinforced Composite, Composites Science and Technology, 68, 2770-2778, 2008.

PINHO, S. T.; DÁVILA, C. G.; CAMANHO, P. P.; IANNUCCI, L.; ROBINSON, P. Failure Models and Criteria for FRP Under In-Plane or Three-Dimensional Stress States Including Shear Non-Linearity, NASA/TM-2005-213530, 2005.

PINHO, S. T.; IANNUCCI, L.; ROBINSON, P. Physically-Based Failure Models and Criteria for Laminated Fibre-Reinforced Composites with Emphasis on Fibre Kinking: Part I: Development. Compos Appl Sci Manuf, 37(1): 63-73, 2006.

PINHO, S. T.; DARVIZEH, R.; ROBINSON, P.; SCHUECKER, C.; CAMANHO, P.P. Material and Structural Response of Polymer-Matrix Fibre-Reinforced Composites, Journal of Composite Materials, Vol. 46, n. 19-20, 2313-2341, 2012.

PUCK, A; SCHÜRMANN, H. Failure Analysis of FRP Laminates by Means of Physically Based Phenomenological Models, Composites Science and Technology, Vol. 58, I. 7, Jul. p. 1045-1067, 1998.

RAND, O.; ROVENSKI, V. Analytical Methods in Anisotropic Elasticity with Symbolic Computational Tools. Boston, Springer, 2005.

ROMANOV, V. S.; LOMOV, S. V.; VERPOEST, I.; GORBATIKH, L. Is It Possible to Eliminate Micro-Scale Stress Concentrations in Composites by Nano Engineering with CNTs? In: Proceedings to $20^{\text {th }}$ INTERNATIONAL CONFERENCE ON COMPOSITE MATERIALS, ICCM 2015, Copenhagen, 2015.

RUSSO, A.; ZUCCARELLO, B. An Accurate Method to Predict the Stress Concentration in Composite Laminates with a Circular Hole Under Tensile Loading, Mechanics of Composite Materials, Vol. 43, No. 4, 2007.

SABUNCOUGLU, B.; ORLOVA, S.; GORBATIKH, L.; LOMOV, S. V.; VERPOEST, I. Micro-Scale Finite Element Analysis of Stress Concentrations in Steel Fiber Composites Under Transverse Loading, Journal of Composite Materials, 0(0) 1-13, 2014.

SADEGHI, G.; HOSSEINI-TOUDESHKY, H.; MOHAMMADI, B. An Investigation of Matrix Cracking Damage Evolution in Composite Laminates: Development of an Advanced Numerical Tool, Composite Structures, 108, 937-950, 2014.

SAVIN, G. N. Stress Distribution Around Holes, NASA Technical Translation, F-607, 1970.

SEVENOIS, R. D. B.; KOUSSIOS, S. Analytic Methods for Stress Analysis of TwoDimensional Flat Anisotropic Plates With Notches: An Overview, Appl. Mech. Rev. Vol. 66, n. 6, 2014. 
SHAH, P. D.; MELO, J. D. D.; CIMINI Jr, C. A.; RIDHA, M. Evaluation of Notched Strength of Composite Laminates for Structural Design, Journal of Composite Materials, Vol. 44, n. 20, 2381-2392, 2010.

SODEN, P. D.; KADDOUR, A. S.; HINTON, M. J. Recommendations for Designers and Researchers Resulting from the World-Wide Failure Exercise, Composites Science and Technology, 64, 589-604, 2004.

SOKOLNIKOFF, I. S. Mathematical Theory of Elasticity. 2. Ed. New York, McGrawHill Book Company, 1956.

SORIANO, E. A.; ALMEIDA, S. F. M. de. Notch Sensitivity of Carbon/Epoxy Fabric Laminates, Composites Science and Technology, 59, 1143-1151, 1999.

SOUTIS, C.; FILIOU, C. Stress Distributions around Holes in Composite Laminates Subjected to Biaxial Loading, Applied Composite Materials, 5: 365-378, 1998.

SU, Z. C.; TAY, T.E.; RIDHA, M.; CHEN, B.Y. Progressive Damage Modeling of OpenHole Composite Laminates Under Compression, Composite Structures, 122, 507-517, 2015.

TAN, S. C. Finite-Width Correction Factors for Anisotropic Plate Containing a Central Opening, Journal of Composite Materials, 22:1080, 1988.

TAN, S. C.; KIM, R. Y. Strain and Stress Concentrations in Composite Laminates Containing a Hole, Experimental Mechanics, Vol. 30, n. 4, 345-351, 1990.

TAN, S. C. Laminated Composites Containing an Elliptical Opening I - Approximate Stress Analyses and Fracture Models, Journal of Composite Materials, Vol. 21, n. 10, 925-948, 1987.

TIMOSHENKO, S.; GOODIER, J. N. Theory of Elasticity. New York: McGraw-Hill, 1951.

TING, T. C. T. Anisotropic Elasticity: Theory and Applications. New York, Oxford University Press, 1996.

TOUBAL, L.; KARAMA, M.; LORRAIN, B. Stress Concentration in a Circular Hole in Composite Plate, Composite Structures, 68, 31-36, 2005.

TSAI, S. W.; MELO, J. D. D. An Invariant-Based Theory of Composites, Composites Science and Technology, 100, 237-243, 2014.

TSAI, S. W.; WU, E. M. A General Theory of Strength for Anisotropic Materials. J. Compos. Mater., 5, 58-80, 1971. 
VASILIEV, V. V.; MOROZOV, E. V. Mechanics and Analysis of Composite Materials, Oxford, Elsevier, 2001.

VAZ, L. E.; PEREIRA, A; MENEZES, I. F. Programação Matemática: Teoria, Algoritmos e Aplicações na Engenharia. Apostila. 2012

VDI Guideline 2014 part 3: Development of Fibre-Reinforced-Plastics Components, Analysis, Berlin, Beuth-Verlag, I. German/English, 2006.

WEI, L.; TING, T. C. T. Explicit Expressions of the Barnett-Lothe Tensors for Anisotropic Materials, Journal of Elasticity, 36: 67-83, 1994.

WEIXING, Y.; XINLU, Y. Stress Concentration Factor for an Orthotropic Finite-Width Plate Containing Elliptical Edge Notches, Composites Science and Technology, Vol. 41, I. 1, p. 47-53, 1991.

WHITNEY, J. M.; NUISMER, R. J. Stress Fracture Criteria for Laminated Composites Containing Stress Concentration, J. Compos. Mater., 8, 253-265, 1974.

XU, X.; WISNOM, M.R.; MAHADIK, Y.; HALLETT, S.R. An Experimental Investigation into Size Effects in Quasi-Isotropic Carbon/Epoxy Laminates with Sharp and Blunt Notches, Composites Science and Technology, 100, 220-227, 2014.

YOUNES, R.; HALLAL, A.; FARDOUN, F.; CHEHADE, F.H. Comparative Review Study on Elastic Properties Modeling for Unidirectional Composite Materials:

Composites and Their Properties. Prof. Ning Hu (Org.), InTech, 2012.

YOUNG, W. C.; BUDYNAS, R. G. Roark's Formulas for Stress and Strain, 7. Ed. [S. 1.]: McGraw-Hill, 2002.

ZAPPALORTO, M.; CARRARO, P. A. An Engineering Formula for the Stress Concentration Factor of Orthotropic Composite Plates, Composites: Part B, 68, 51-58, 2015. 


\section{APÊNDICE A - Concentração de Tensão em uma Placa Anisotrópica com Furo Circular}

O objetivo dessa seção é apresentar uma forma simplificada que facilita a implementação para casos de furos circulares, que são os mais comuns na prática. A utilidade de se estudar furos elípticos é pela sua capacidade de aproximar outros problemas e sua aplicação no estudo de furos circulares com danos (também aproximado).

Para se modelar o furo circular também se faz uso de mapeamento conforme. Todavia, a equação final é bem mais compacta pela simplicidade do mapeamento. Para um furo circular de raio $r$, pode-se escrever sua equação paramétrica como

$$
\begin{aligned}
& x_{1}=r \cos \psi \\
& x_{2}=r \sin \psi
\end{aligned}
$$

Usando a transformação mostrada na Equação (45), mas agora fazendo $\zeta=e^{i \psi}=\cos \psi+i \sin \psi$, pode-se escrever a seguinte relação:

$$
\begin{aligned}
& r \cos \psi+p_{\xi} r \sin \psi=\left(c_{\xi}+d_{\xi}\right) \cos \psi+\left(c_{\xi}-d_{\xi}\right) i \sin \psi \\
& \text { Logo } \\
& c_{\xi}=\frac{r\left(1-i p_{\xi}\right)}{2} \\
& d_{\xi}=\frac{r\left(1+i p_{\xi}\right)}{2}
\end{aligned}
$$

Supondo as soluções dos campos de deslocamentos e das funções de tensões da mesma forma que foram mostradas no Capítulo 2, pode-se utilizar as Equações (48) e (50). As condições de contorno representadas pela Equação (49) também devem continuar sendo válidas e vetor q pode ser então calculado como

$$
\mathbf{q}=-\frac{1}{2} \mathbf{W}^{-1}\left(\boldsymbol{\tau}_{2}-i \boldsymbol{\tau}_{1}\right) r
$$

E a solução da função de tensão pode ser reescrita como

$$
\boldsymbol{\phi}=x_{1} \boldsymbol{\tau}_{2}-x_{2} \boldsymbol{\tau}_{1}-\operatorname{Re}\left\{\left(\mathbf{W}\left\langle\zeta_{\xi}^{-1}\right\rangle \mathbf{W}^{-1}\right)\left(\boldsymbol{\tau}_{2}-i \boldsymbol{\tau}_{1}\right) r\right\}
$$

E a sua derivada em relação ao vetor unitário tangente a borda do furo é 
$\frac{\partial \phi}{\partial \mathbf{e}_{2}^{(l)}}=\left(\mathbf{G}_{1}^{(l)} \boldsymbol{\tau}_{1}-\left(\mathbf{I}+\mathbf{G}_{3}^{(l)}\right) \boldsymbol{\tau}_{2}\right) \sin \theta-\left(\left(\mathbf{I}+\mathbf{G}_{3}^{(l)}\right) \boldsymbol{\tau}_{1}+\mathbf{G}_{1}^{(l)} \boldsymbol{\tau}_{2}\right) \cos \theta$

onde I é a matriz identidade 3x3. Por último, utilizando a Equação (56), obtêm-se $\sigma_{11}^{(l)}=\mathbf{i}_{\mathbf{1}}\left(\mathbf{G}_{1}^{(l)} \boldsymbol{\tau}_{2}+\mathbf{G}_{3}^{(l)} \boldsymbol{\tau}_{1}\right)-\mathbf{i}_{2}\left(\mathbf{G}_{1}^{(l)} \boldsymbol{\tau}_{1}-\mathbf{G}_{3}^{(l)} \boldsymbol{\tau}_{2}\right)$

onde $\mathbf{i}_{1}=\left[\begin{array}{lll}1 & 0 & 0\end{array}\right]$ e $\mathbf{i}_{2}=\left[\begin{array}{lll}0 & 1 & 0\end{array}\right]$. Note que a Equação (A.A.7) reproduz o resultado desenvolvido na seção 2 para $r_{a}=r_{b}=r$, mas tem uma equação final que pode ser expressa de forma muito mais simples e compacta, o que facilita sua aplicação. 


\section{APÊNDICE B - Relação Entre Notações}

A notação tensorial é útil e elegante porque apresenta de forma compacta as equações, mas não é uma ferramenta tão popular na engenharia quanto as matrizes. Para expandir a aplicabilidade do presente texto, será apresentada a seguir a equivalência entre ambas as notações. Por simplicidade, serão mostradas apenas as transformações da matriz das tensões e da matriz de flexibilidade, que são tensores de segunda e quarta ordem, respectivamente, e será considerado que se deseja transformar uma matriz definida em relação ao sistema de coordenada dos materiais para o sistema de coordenadas genérico.

Denotando por $x_{i}^{*}$ o sistema de coordenadas que se deseja obter as transformações e que seja feita uma rotação $\theta$ no plano $x_{1}-x_{2}$ e uma rotação $\phi$ no plano $x_{1}-x_{3}$, as tensões em coordenadas locais podem ser escritas como (CASTRO; MEGGIOLARO, 2016b)

$$
\boldsymbol{\sigma}^{*}=\boldsymbol{\Omega} \boldsymbol{\boldsymbol { \Omega } ^ { T }}
$$

onde

$$
\boldsymbol{\Omega}=\left[\begin{array}{ccc}
\cos \theta \cos \phi & \sin \theta \cos \phi & -\sin \phi \\
-\sin \theta & \cos \theta & 0 \\
\sin \phi & \sin \phi & \cos \phi
\end{array}\right]
$$

A transformação das tensões é direta por ser um tensor de ordem dois, tornando simples a relação matricial. Para a matriz de flexibilidade, que é um tensor de quarta ordem, a transformação é escrita como (Ting, 1996)

$$
c^{*}=\left(\boldsymbol{K}^{-1}\right)^{T} \boldsymbol{c} \boldsymbol{K}^{-1}
$$

onde

$$
\begin{aligned}
& \boldsymbol{K}=\left[\begin{array}{ll}
\boldsymbol{K}_{1} & 2 \boldsymbol{K}_{2} \\
\boldsymbol{K}_{3} & \boldsymbol{K}_{4}
\end{array}\right] \\
& \boldsymbol{K}_{1}=\left[\begin{array}{lll}
\Omega_{11}{ }^{2} & \Omega_{12}{ }^{2} & \Omega_{13}{ }^{2} \\
\Omega_{21}{ }^{2} & \Omega_{22}{ }^{2} & \Omega_{23}{ }^{2} \\
\Omega_{31}{ }^{2} & \Omega_{32}{ }^{2} & \Omega_{33}{ }^{2}
\end{array}\right] \\
& \boldsymbol{K}_{2}=\left[\begin{array}{lll}
\Omega_{12} \Omega_{13} & \Omega_{13} \Omega_{11} & \Omega_{11} \Omega_{12} \\
\Omega_{22} \Omega_{23} & \Omega_{23} \Omega_{21} & \Omega_{21} \Omega_{22} \\
\Omega_{32} \Omega_{33} & \Omega_{33} \Omega_{31} & \Omega_{31} \Omega_{32}
\end{array}\right]
\end{aligned}
$$




$$
\begin{aligned}
\boldsymbol{K}_{3} & =\left[\begin{array}{lll}
\Omega_{21} \Omega_{31} & \Omega_{22} \Omega_{32} & \Omega_{23} \Omega_{33} \\
\Omega_{31} \Omega_{11} & \Omega_{32} \Omega_{12} & \Omega_{33} \Omega_{13} \\
\Omega_{11} \Omega_{21} & \Omega_{12} \Omega_{22} & \Omega_{13} \Omega_{23}
\end{array}\right] \\
\boldsymbol{K}_{4} & =\left[\begin{array}{lll}
\Omega_{22} \Omega_{33}+\Omega_{23} \Omega_{32} & \Omega_{23} \Omega_{31}+\Omega_{21} \Omega_{33} & \Omega_{21} \Omega_{32}+\Omega_{22} \Omega_{31} \\
\Omega_{32} \Omega_{13}+\Omega_{33} \Omega_{12} & \Omega_{33} \Omega_{11}+\Omega_{31} \Omega_{13} & \Omega_{31} \Omega_{12}+\Omega_{32} \Omega_{11} \\
\Omega_{12} \Omega_{23}+\Omega_{13} \Omega_{22} & \Omega_{13} \Omega_{21}+\Omega_{11} \Omega_{23} & \Omega_{11} \Omega_{22}+\Omega_{12} \Omega_{21}
\end{array}\right]
\end{aligned}
$$

Uma forma alternativa da equação (A.B.1) é (Ting, 1996)

$$
\boldsymbol{\sigma}^{*}=\boldsymbol{K} \boldsymbol{\sigma}
$$




\section{ANEXO I - Lista de Publicações}

VIGNOLI, L. L.; CASTRO, J. T. P. ; MEGGIOLARO, M. A. . Stress Concentration Analysis in Orthotropic Plates. In: 23rd International Congress of Mechanical Engineering, 2015, Rio de Janeiro. COBEM 2015, 2015.

VIGNOLI, L. L.; CASTRO, J. T. P. ; MEGGIOLARO, M. A. . Modelling Stress Concentration Effect in Composite Plates. In: 3rd Brazilian Conference on Composite Materials, Gramado. BCCM-3, 2016.

VIGNOLI, L. L.; CASTRO, J. T. P. . Resistência de Laminado Angle Ply com Concentração de Tensão. In: $71^{\circ}$ Congresso Anual da ABM, Rio de Janeiro. ABM 2016, 2016. 


\section{ANEXO II - Envelopes de Falha do WWFE}

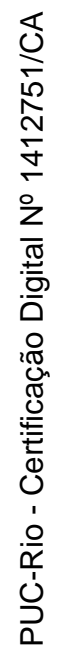

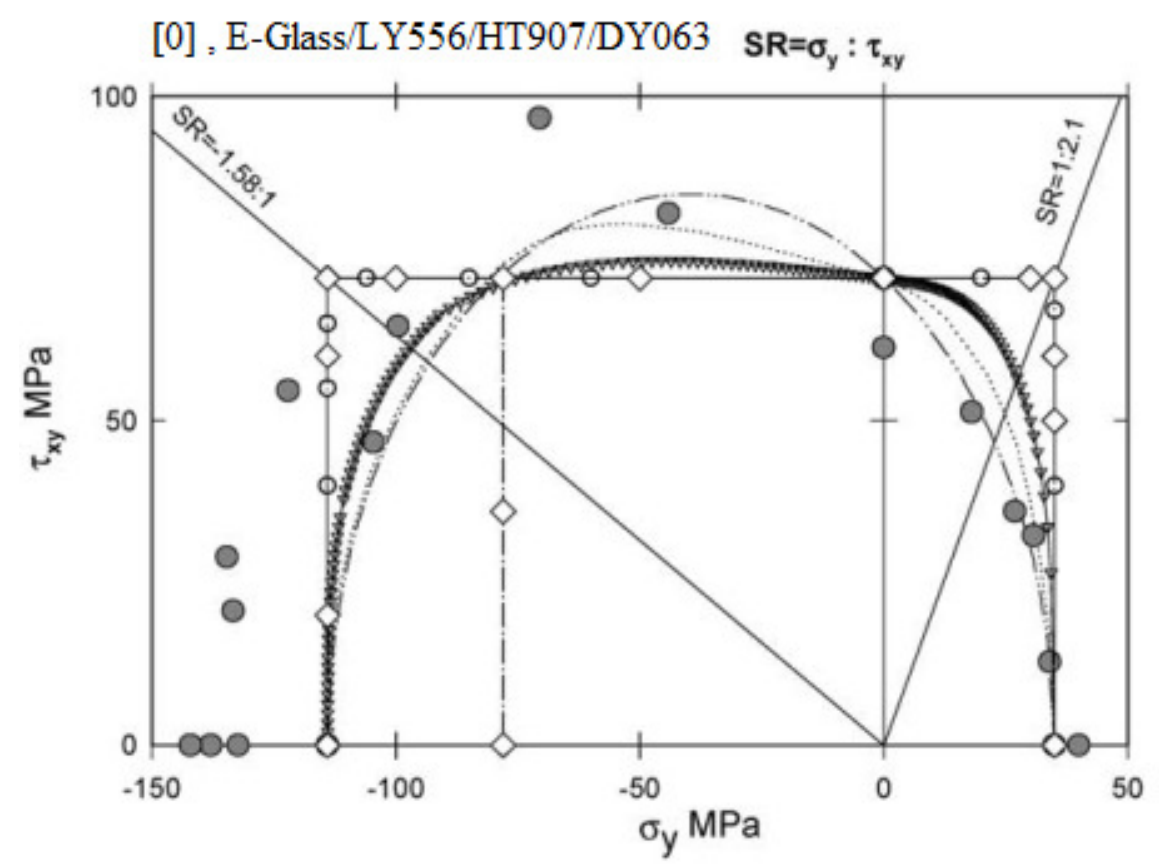

$\begin{array}{lllll}-\cdots & \text { Tsai } \odot \multimap \text { Zinoviev } \diamond \diamond \text { Bogetti } \\ \cdots & \text { Puck } \rightarrow \text { Cuntze } & \diamond & \text { Test results }\end{array}$
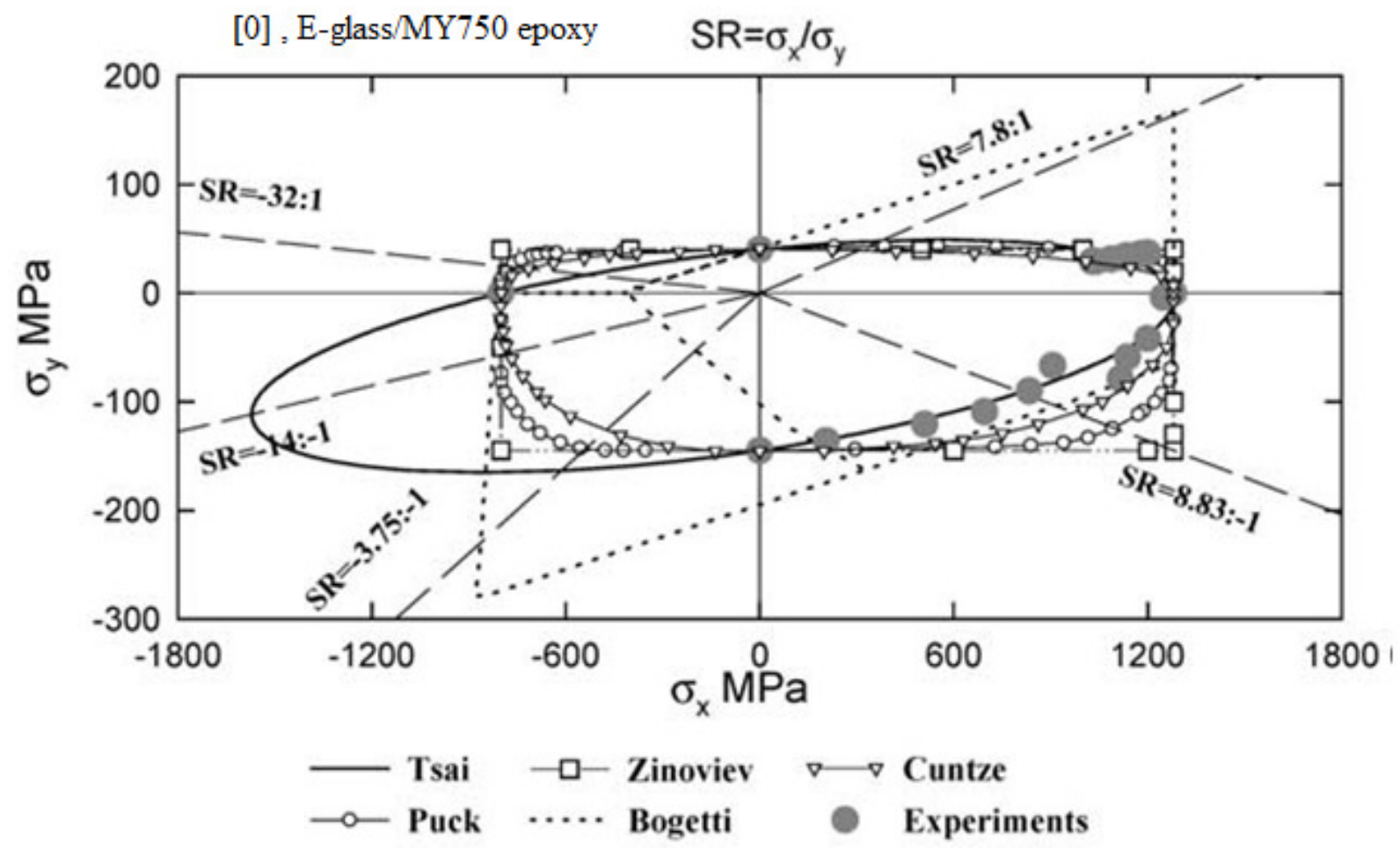

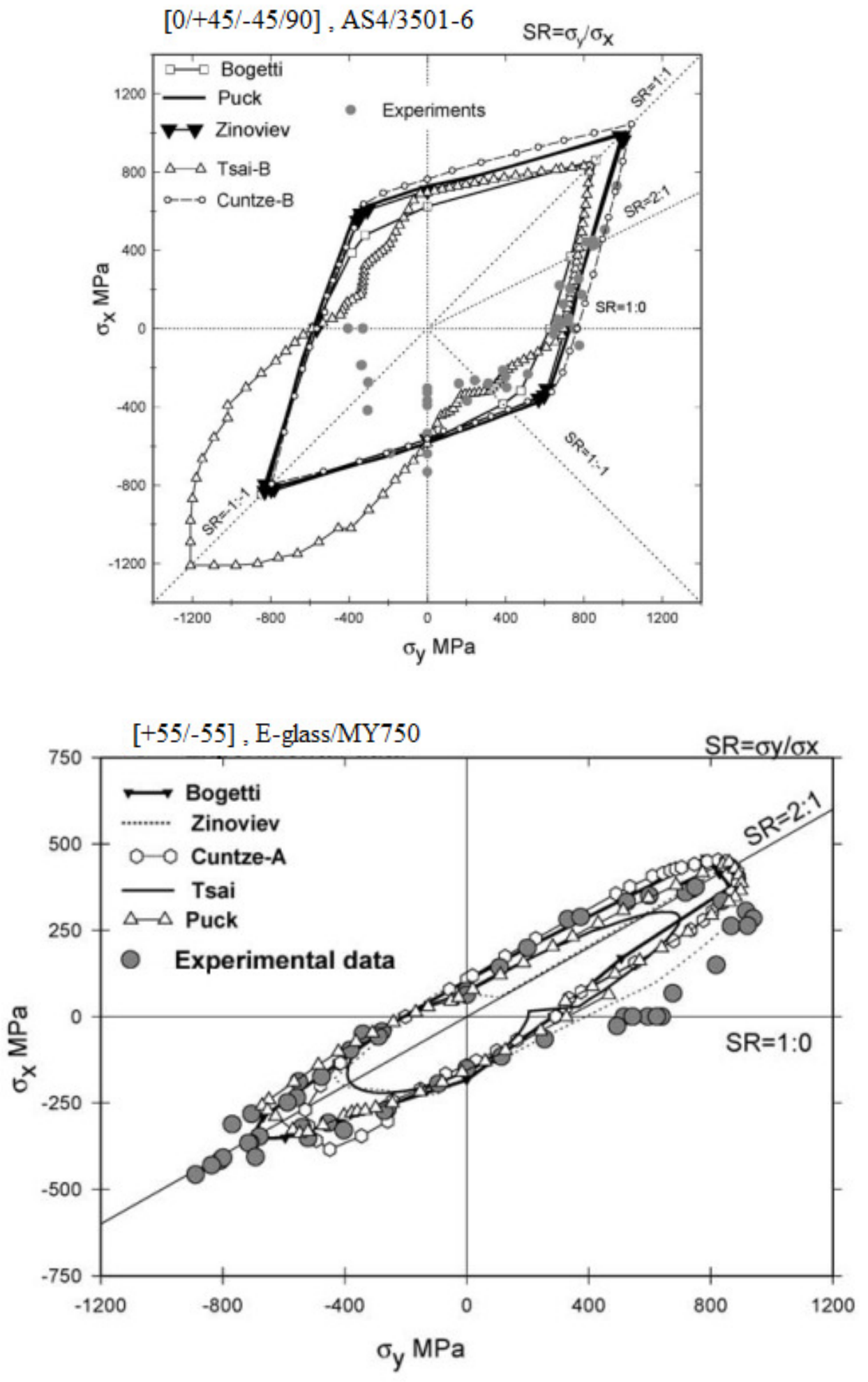

Figura 61: Envelopes de falha do WWFE I (SODEN et al., 2004 e HINTON et al., 2004) 
$0^{\circ}$

T300/PR319

Stress ratio $S R=p r e s s u r e:$ shesr stress $=P: \tau_{12}$
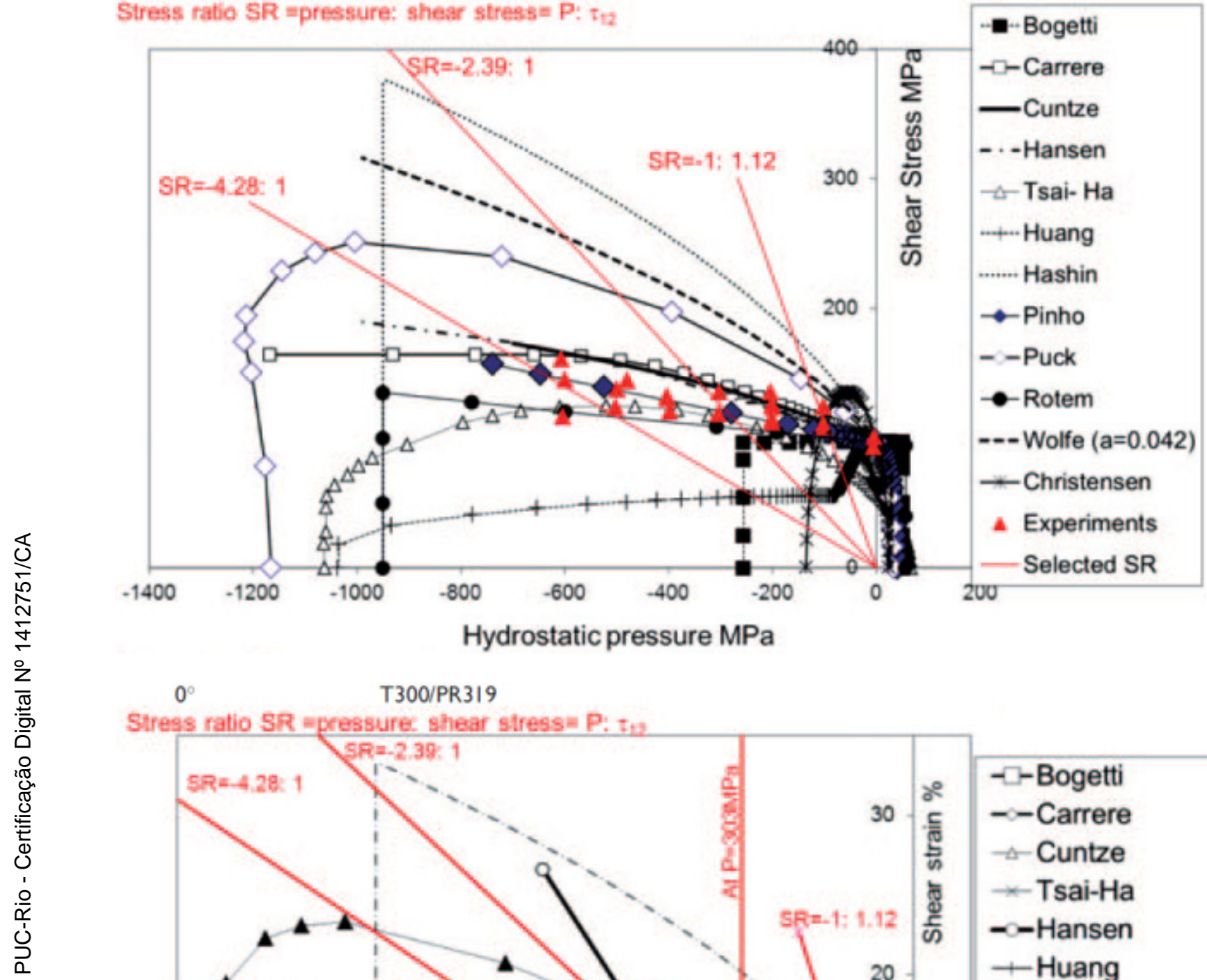

Hydrostatic pressure MPa

$0^{\circ}$

T300/PR319

Stress ratio $S R$ =pressure: shear stress= P: tw

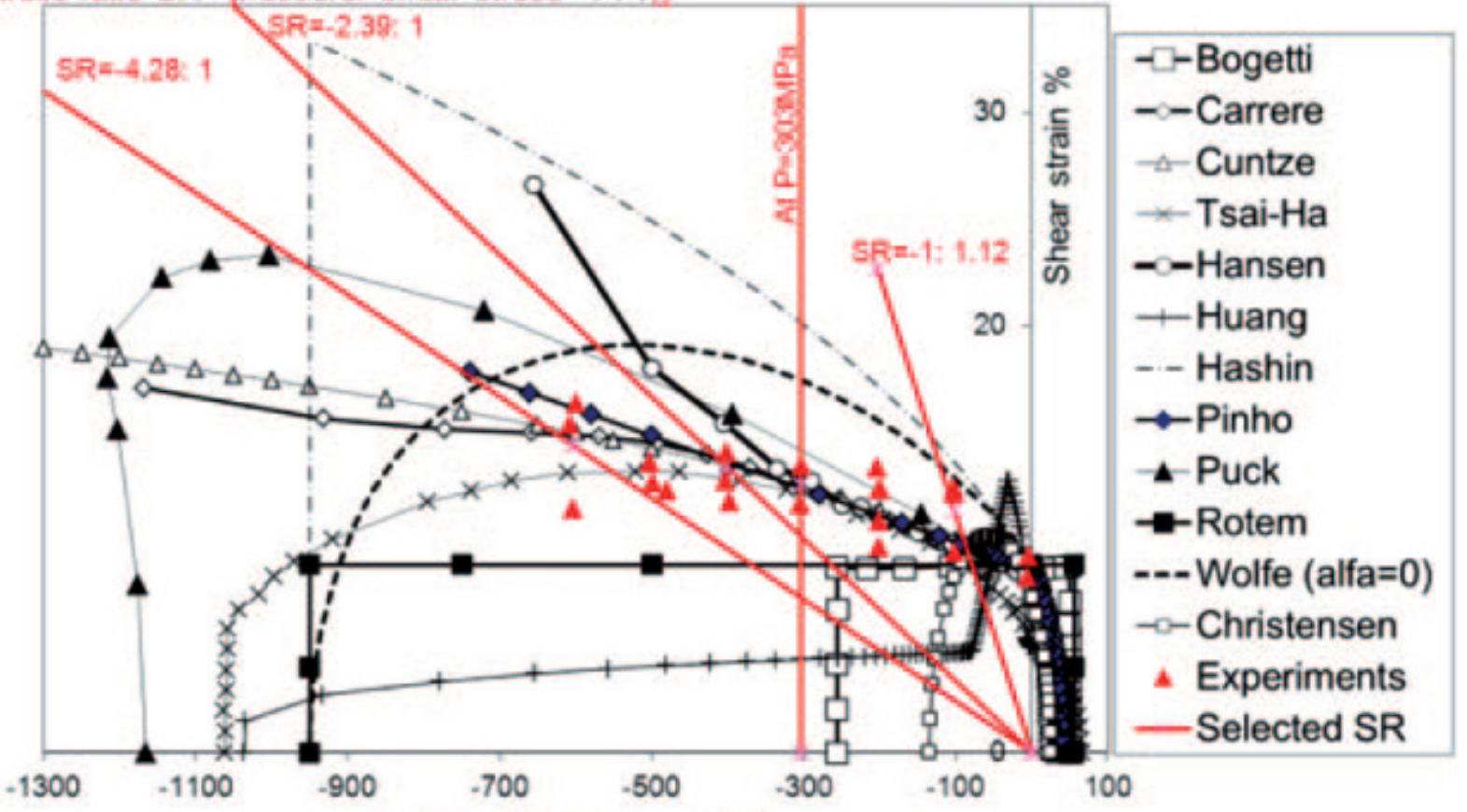

Hydrostatic pressure MPa 


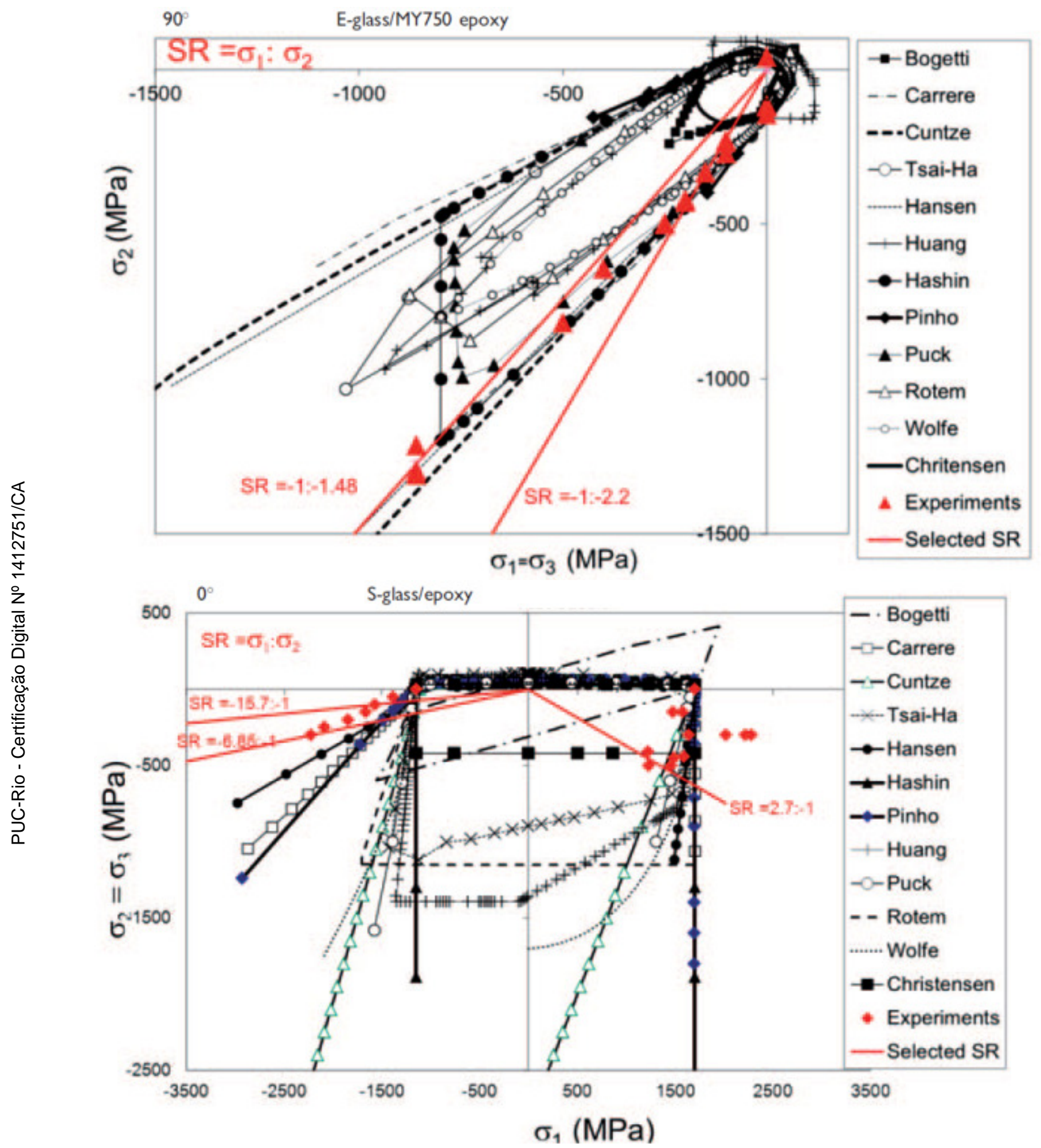




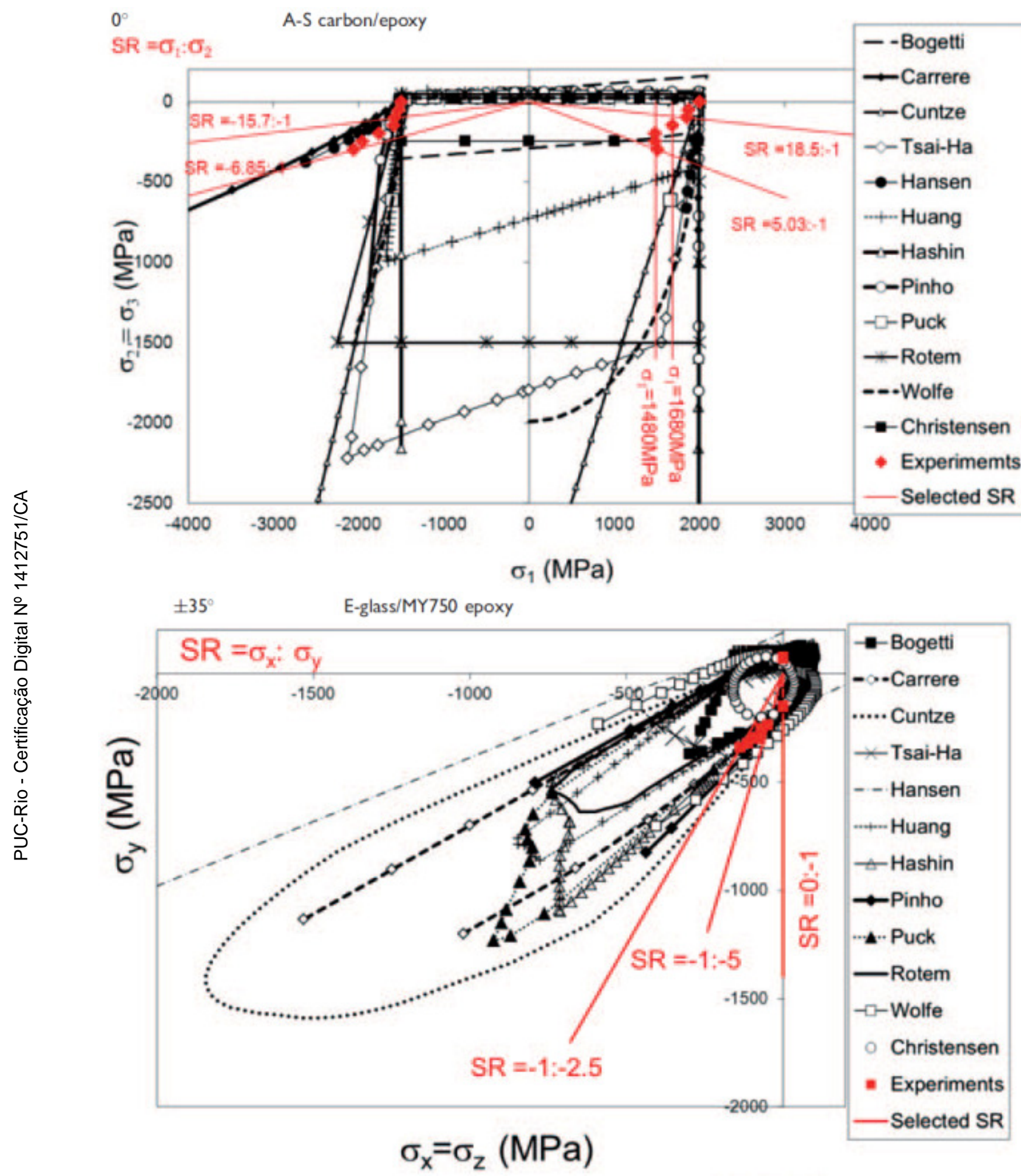




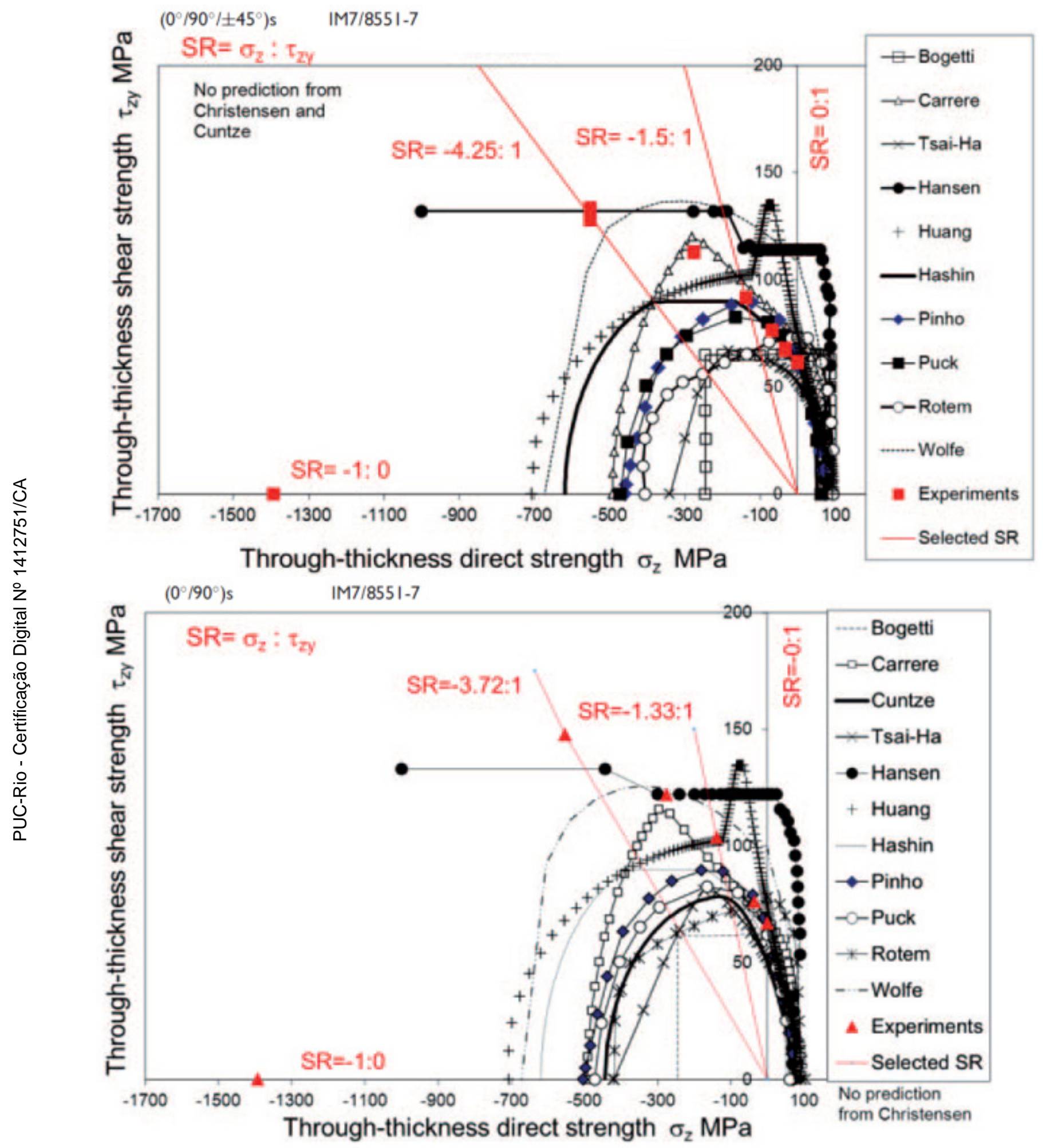

Figura 62: Envelopes de falha do WWFE II (Kaddour e Hinton, 2013) 